\title{
Vascular plant species richness at the landscape scale: Patterns and processes
}

\author{
Dissertation \\ zur Erlangung des mathematisch-naturwissenschaftlichen Doktorgrades \\ "Doctor rerum naturalium" \\ der Georg-August-Universität Göttingen \\ im Promotionsprogramm Biologie \\ der Georg-August University School of Science (GAUSS)
}

vorgelegt von

Inga Schmiedel

aus Göttingen

Göttingen, 2014 


\section{Betreuungsausschuss}

Prof. Dr. E. Bergmeier, Abt. Vegetationsanalyse \& Phytodiversität, Georg-AugustUniversität Göttingen

Prof. Dr. C. Leuschner, Abt. Ökologie \& Ökosystemforschung, Georg-AugustUniversität Göttingen

Dr. H. Culmsee, Abt. Vegetationsanalyse \& Phytodiversität, Georg-August-Universität Göttingen

Mitglieder der Prüfungskommission

Referent: Prof. Dr. E. Bergmeier, Abt. Vegetationsanalyse \& Phytodiversität, GeorgAugust-Universität Göttingen

Korreferent: Prof. Dr. C. Leuschner, Abt. Ökologie \& Ökosystemforschung, GeorgAugust-Universität Göttingen

\section{Weitere Mitglieder der Prüfungskommission}

Prof. Dr. H. Behling, Abt. Palynologie \& Klimadynamik, Albrecht-von-Haller-Institut für Pflanzenwissenschaften, Georg-August-Universität Göttingen

Prof. Dr. S. Bögeholz, Didaktik der Biologie, Albrecht-von-Haller-Institut für Pflanzenwissenschaften, Georg-August-Universität Göttingen

Prof. Dr. R. Bürger-Arndt, Abt. für Naturschutz und Landschaftspflege, BurckhardtInstitut, Georg-August-Universität Göttingen

Prof. Dr. M. Hauck, Abt. Ökologie \& Ökosystemforschung, Albrecht-von-HallerInstitut für Pflanzenwissenschaften, Georg-August-Universität Göttingen

Tag der mündlichen Prüfung: 18.12.2014 


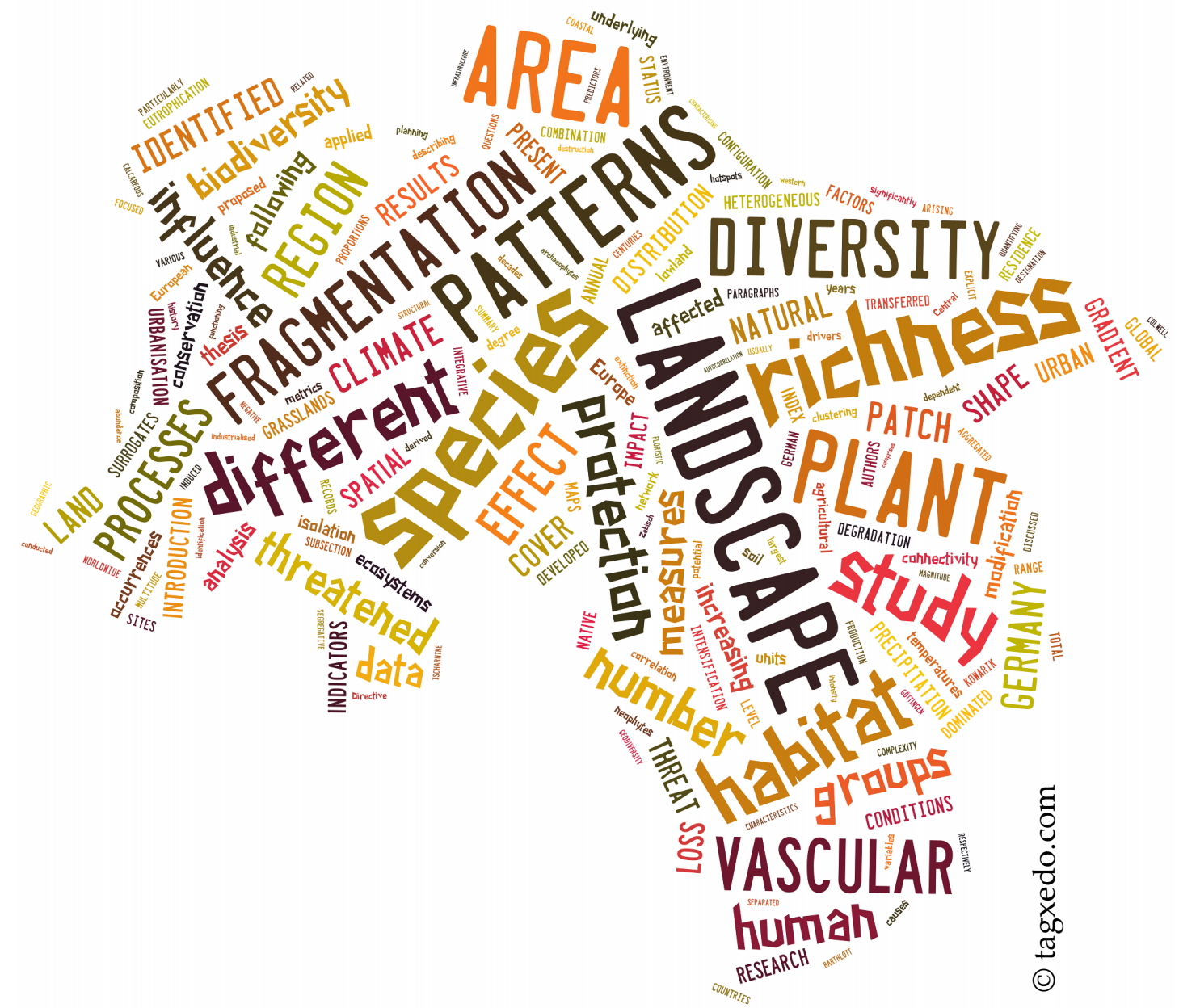





\section{Contents}

List of Figures I

List of Tables III

Abbreviations $\quad$ V

$\begin{array}{ll}\text { Summary } & 1\end{array}$

Zusammenfassung 3

1 Introduction $\quad \mathbf{7}$

1.1 Patterns of species richness at the landscape scale $\ldots \ldots \ldots$

1.2 Linking patterns and processes: What influences species richness patterns at the landscape scale? . . . . . . . . . . . . . . . . . . 9

1.2.1 Environmental conditions as key drivers for richness patterns of plants........................ 10

1.2.2 The human impact on richness patterns of plants . . . . . . 10

1.3 General objectives and thesis outline . . . . . . . . . . . . . 17

2 Description of data 33

2.1 Study region . . . . . . . . . . . . . . . . . . . 34

2.2 Data and data preparation . . . . . . . . . . . . . . . 35

2.2 .1 Species data . . . . . . . . . . . . . . . . . . 35

2.2 .2 ATKIS data . . . . . . . . . . . . . . . . . . . . 37

2.2.3 Areal information . . . . . . . . . . . . . . . . . . 40

2.2.4 Environmental variables . . . . . . . . . . . . . . . . . . . 40

2.2 .5 Landscape metrics . . . . . . . . . . . . . . . . . . 41

3 Patterns of vascular plant species richness 51

4 The effectiveness of protected areas for the conservation of threatened plants 77

5 The influence of landscape fragmentation on richness patterns of plants 99

6 Plant species richness along a gradient of landscape modification 129 
7 Synthesis $\quad 155$

7.1 Key findings and implications . . . . . . . . . . . . . . . . . . 156

7.1.1 Distinct patterns of vascular plant species richness in Lower Saxony and Bremen . . . . . . . . . . . . . . . 156

7.1.2 Effective 'territorial' protection of hotspots of threatened plant species . . . . . . . . . . . . . . . . . 157

7.1.3 Human impact matters: Plant species richness along gradients of landscape modification and fragmentation . . . . . . . . 158

7.1.4 Overall key findings derived from the studies . . . . . . . . . . 159

7.2 Transferability of the methodological approach . . . . . . . . . . . . . . . 160

7.3 Limitations of landscape-level approaches . . . . . . . . . . . . . 163

7.3.1 Bias due to generalisations, data resolutions and spatial scale . . 163

7.3.2 Bias due to data format . . . . . . . . . . . . . . . 165

7.3 .3 Bias in species records . . . . . . . . . . . . . . 165

7.4 Concluding remarks and outlook . . . . . . . . . . . . . . 166

$\begin{array}{ll}\text { Acknowledgements } & 175\end{array}$

$\begin{array}{lr}\text { Academic CV } & 177\end{array}$

$\begin{array}{ll}\text { List of publications } & 179\end{array}$

$\begin{array}{lr}\text { Appendix A } & 181\end{array}$

$\begin{array}{lr}\text { Appendix B } & 217\end{array}$ 


\section{List of Figures}

1.1 Human-induced threats to the local species pool. . . . . . . . . . . . . 14

2.1 The study area with its three natural landscape regions. . . . . . . . 36

3.1 Das Untersuchungsgebiet umfasst die Gesamtfläche der Bundesländer Niedersachsen und Bremen und wird in drei naturräumliche Großregionen unterteilt. . . . . . . . . . . . . . . . . . . 59

3.2 Anzahl der in Niedersachsen und Bremen vorkommenden Gefäßpplanzensippen mit floristischem Normalstatus je Quadrant. . . . . . . . . . 63

3.3 Anzahl der in Niedersachsen und Bremen vorkommenden 1.509 indigenen Gefäßpflanzensippen. . . . . . . . . . . . . . . . . . . . 67

3.4 Sippenzahl je Quadrant der in Niedersachsen und Bremen vorkommenden 160 Archäophyten. . . . . . . . . . . . . . . . . . . . 68

3.5 Sippenzahl je Quadrant der in Niedersachsen und Bremen vorkommenden 145 etablierten Neophyten. . . . . . . . . . . . . . . . . . 69

3.6 Anzahl der in Niedersachsen und Bremen je Quadrant vorkommenden 1176 ungefährdeten Gefäßpflanzensippen. . . . . . . . . . . . . . . 71

3.7 Anzahl der 643 seltenen und gefährdeten Gefäßpflanzensippen Niedersachsens und Bremens je Quadrant. . . . . . . . . . . . . . . . 72

4.1 Das Untersuchungsgebiet des niedersächsischen Tieflandes mit seinen fünf naturräumlichen Großregionen und 41 naturräumlichen Haupteinheiten. . . . . . . . . . . . . . . . . . . . 84

4.2 Anzahl der seltenen und gefährdeten Gefäßpflanzensippen pro Minutenfeld im niedersächsischen Tiefland. . . . . . . . . . . . . . . . . 88

4.3 Anzahl der seltenen und gefährdeten Waldgefäßpflanzensippen pro Minutenfeld im niedersächsischen Tiefland. . . . . . . . . . . . . . 89

4.4 Schutzgebietsflächen (in Hektar) in den fünf Großregionen des niedersächsischen Tieflands unter Berücksichtigung der räumlichen Überlagerung von NSG und FFH-Gebieten. . . . . . . . . . . . . . 90

4.5 Beispiele für die Korrelation zwischen Schutzgebietsausdehnung und den Vorkommen der RL-Arten bzw. RL-Waldarten je Minutenfeld. . . . 92

4.6 Habitatbindung der seltenen und gefährdeten Waldgefäßpflanzenarten des Tieflandes . . . . . . . . . . . . . . . . . . . . . . . . . . . . 94 
5.1 The state of Lower Saxony, Germany, with its three major landscape components. . . . . . . . . . . . . . . . . . . . . . 104

5.2 Example of one of 1386 landscape units in Lower Saxony, Germany. . 107

5.3 Fractions obtained from variation partitioning based on three variables (Venn diagram). . . . . . . . . . . . . . . . . . . . . 110

5.4 Frequency distribution of the five $m_{\text {eff }}$ types for the sample of 1386 landscape units (grid cells) in Lower Saxony, Germany. . . . . . . . . . . 111

6.1 The state of Lower Saxony, Germany, with its three major landscape components. . . . . . . . . . . . . . . . . . . 135

6.2 Differences in vascular plant species richness between different clusters resulting from k-means cluster analysis of 1383 landscape units in Lower Saxony, Germany. . . . . . . . . . . . . . . . . . . . . . . . . . 143 


\section{List of Tables}

2.1 Overview of the data used in the analysis. . . . . . . . . . . . 38

2.2 Groups of vascular plant species used in the analyses. . . . . . . . . . 39

2.3 Land cover types derived from ATKIS-DLM. . . . . . . . . . . . . . . . 41

2.4 Landscape metrics used to assess landscape composition with respect to their range of values, units and basis of computation. . . . . . . . . 43

2.5 Landscape metrics used to assess landscape configuration with respect to their range of values, units and basis of computation. . . . . . . . 45

3.1 Gesamtsippenzahl, mittlere Sippenzahl je Quadrant und maximale Sippenzahl pro Quadrant für verschiedene Artengruppen für Niedersachsen und Bremen. . . . . . . . . . . . . . . . . . . . . . . 62

3.2 Auflistung der jeweils fünf sippenreichsten Quadranten je Artengruppe. 64

3.3 Moran's Index als Maß für die räumliche Autokorrelation der Phytodiversitätszentren für die verschiedenen Gruppen. . . . . . . . . . . . . . 65

3.4 Ähnlichkeiten in den Verbreitungsschwerpunkten der Gruppen nach Einbürgerungsstatus bzw. Gefährdungssituation. . . . . . . . . . . . 66

3.5 Zahl der Gefäßpflanzensippen nach ihrem Einbürgerungsstatus und ihrer jeweiligen Gefährdungssituation. . . . . . . . . . . . . . . 70

4.1 Gesamtfläche, absolute und prozentuale Flächenanteile von Schutzgebieten und Waldschutzgebieten. . . . . . . . . . . . . . 86

4.2 Ergebnisse der Effektivitäts-Analyse. . . . . . . . . . . . . . . . . . . . 91

5.1 Number of vascular plant species in Lower Saxony, Germany, grouped by their residence and threat status. . . . . . . . . . . . . . . . . 105

5.2 Landscape fragmentation variables, spatial and environmental co-variables used in this study. . . . . . . . . . . . . . . . . . 108

5.3 Results of variation partitioning for vascular plant species richness in Lower Saxony, Germany. . . . . . . . . . . . . . . . . . . . . 113

5.4 Correlation of the $m_{\text {eff }}$ types MeshFG1 and MeshFG2, respectively, with species richness of six species groups assessed for Lower Saxony, Germany. . . . . . . . . . . . . . . . . . . . . . . . . . . . . . . 114

5.5 Overview of studies using the Effective Mesh Size Index to quantify landscape fragmentation. . . . . . . . . . . . . . . 116

6.1 Landscape metrics derived from land cover data. . . . . . . . . . 136 
6.2 Total number of vascular plant species of Lower Saxony, Germany, and the number of species of seven sub-groups distinguished by residence status, threat status and habitat preferences. . . . . . . . . . . . . . 139

6.3 Characterisation of the landscapes in the six clusters found for 1383 landscape units in Lower Saxony, Germany. . . . . . . . . . . . . . . 141

6.4 Scores of the first ordination axis of a PCA using the standardized landscape metrics calculated for 1383 landscape units in Lower Saxony, Germany. . . . . . . . . . . . . . . . . . . . . . . . . . . . . . . . . . 144

6.5 Levels of landscape modification found for 1383 landscape units in Lower Saxony, Germany. . . . . . . . . . . . . . . . . . . . . . . 147

7.1 Overview of the largest data bases on the distribution of vascular plant species in Europe. . . . . . . . . . . . . . . . . . . . . 162

A.1 Bio-geographical regions and natural landscape units of Lower Saxony. 181

A.2 List of vascular plant species of the data base of the vascular plant survey of Lower Saxony. . . . . . . . . . . . . . . . . . . . . 183

A.3 Codes and full names of (sub-)species assigned to the species and/or aggregate of higher taxonomical level. . . . . . . . . . . . . . 206

A.4 Soil types listed in the soil map of Lower Saxony and the assignment to 27 soil type categories. . . . . . . . . . . . . . . . . . . . 209

A.5 Roads that are represented as polylines in the ATKIS-DLM were buffered by using the buffer distance specified below. . . . . . . . . . . . . . 212

A.6 Land cover types used for the calculation of landscape metrics. . . . . . 213

A.7 Characterisation of the landscapes in the six clusters found for 1383 landscape units in Lower Saxony, Germany. . . . . . . . . . . . . . . . 214

A.8 Overlap of species sub-groups for which the richness patterns along the gradient of landscape modification intensity for 1383 landscape units in Lower Saxony, Germany, were analysed. . . . . . . . . . . . . . . . . . 215

A.9 Species richness per cluster for the total number of species occurring in 1383 landscape units in Lower Saxony, Germany. . . . . . . . . . . . . . 215 


\section{Abbreviations}

ATKIS Authorative Topographic-Cartographic Information System, Amtliches Topographisch-Kartographisches Informationssystem

Az. Reference number, Aktenzeichen

BfN Federal Agency for Nature Conservation, Bundesamt für Naturschutz

BMU Federal Ministry for the Environment, Nature Conservation, Building and Nuclear Safety, Bundesministeriums für Umwelt, Naturschutz, Bau und Reaktorsicherheit

BNatSchG Federal Nature Conservation Act, Bundesnaturschutzgesetz

BÜK 50 Soil map, scale 1:50,000, Bodenübersichtskarte 1:50,000

$\mathrm{CaCO}_{3}$ Calcium carbonate

CBD Convention on Biological Diversity

COP Convention of the Parties

DBU German Federal Foundation for the Environment, Deutsche Bundesstiftung Umwelt

DEM Digital Elevation Model

DHDN Deutsches Hauptdreiecksnetz (coordinate system)

DLM Digital Landscape Model

DWD German Weather Service, Deutscher Wetterdienst

EEA European Environment Agency

e.g. For example [lat. exempli gratia]

EIV Ellenberg Indicator Values

EU European Union

FFH Fauna-Flora-Habitat (Habitats Directive)

FG Fragmentation geometry

FGSV Road Research Institute, Forschungsgesellschaft für Straßen- und Verkehrswesen 


\section{GBIF Global Biodiversity Information Facility \\ GIS Geographic Information System \\ GLM Generalised Linear Model \\ GSPC Global Strategy for Plant Conservation}

i.e. that means [lat. id est]

IPA Important Plant Areas

LCT Land cover type

LGN Lower Saxon Authority for Land-surveying and Geoinformation, Landesvermessung und Geobasisinformation Niedersachsen

$\mathbf{m}_{\text {eff }} \quad$ Effective Mesh Size Index

n/a not available

NLfB Lower Saxon Regional Authority for Mining, Power and Geology, Landesamt für Bergbau, Energie und Geologie

NLÖ Lower Saxon Regional Authority of Ecology, Niedersächsisches Landesamt für Ökologie

NLWKN Lower Saxon Water Management, Coastal Defence and Nature

Conservation Agency, Niedersächsisches Landesamt für Wasserwirtschaft, Küsten und Naturschutz

NMUK Lower Saxon Ministry for the Environment and Climate Protection, Niedersächsischen Ministerium für Umwelt, Energie und Klimaschutz

NR Geographical regions, naturräumliche Regionen

NSG Nature protection area, nature reserve, Naturschutzgebiet

NW-FVA Northwest German Forest Research Station, Nordwestdeutsche Forstliche Versuchsanstalt

RL $\quad$ Red List

UG Study area, Untersuchungsgebiet

UNECE United Nations Economic Commission for Europe

VEAC Victorian Environmental Assessment Council

z. B. German for zum Beispiel (for example) 


\section{Summary}

The heterogeneous geographic distribution of species richness has fascinated and challenged biologists over centuries. As a result, a large number of possible hypotheses that aim at explaining these patterns have been proposed. Currently, the consensus is that multiple processes simultaneously affect species richness patterns, with the influence of each process differing between taxa, landscapes and periods in time. Generally, environmental conditions are assumed to be the main drivers underlying species richness patterns at large scales. However, as many landscapes and thus species' habitats are strongly influenced by humans, particularly in Central Europe, the effect of human land use on richness patterns cannot be neglected. The severe anthropogenic changes in land cover, increasing landscape fragmentation, degradation and intensification have already led to large losses in (semi-)natural vegetation, biodiversity and ecosystem functions and are expected to cause even more losses in the future.

Protected areas play a major role in the alleviation of anthropogenic pressures on species and their habitats. However, as site selection for many of these areas has not been based on knowledge on species occurrences, their effectiveness might be limited.

The present thesis, conducted in the federal states of Lower Saxony and Bremen in northern Germany, therefore aims at 1.) identifying the richness patterns of vascular plant species in the two states; 2 .) testing the effectiveness of the protected area network for the conservation of threatened species in the lowland region of the study area and 3.) analysing the relationship between species richness patterns and their underlying processes with special regard to the human influence.

In order to address these aims, analyses were carried out on grid-based floristic data obtained from the vascular plant survey of Lower Saxony (Niedersächsisches Pflanzenartenerfassungsprogramm, NLWKN 1982-2003) that were combined with highresolution data on land cover and information on environmental conditions and the location and extent of protected areas. In doing so, the thesis represents the first comprehensive analysis of the extensive floristic data base of Lower Saxony.

To analyse the patterns of vascular plant species richness across the study region, the overall species richness as well as the richness of five sub-groups of species aggregated using information on residence and threat status of the species inside each landscape unit $\left(1,762\right.$ grid cells of c. $\left.30 \mathrm{~km}^{2}\right)$ was assessed. Richness of all species groups was found to be heterogeneously distributed across the region showing an increase in richness from north to south and - to some extent - also from west to east. The centres of high plant species richness of the different groups largely correlated with each other but also showed some interesting divergences. 
The effectiveness of the protected area network in the lowland region of the state was shown to be generally high: 'Hotspots' of threatened species were found to be largely covered by nature reserves (German: Naturschutzgebiete) and Natura 2000 sites with the latter areas expediently complementing the former. It remained unclear, however, if the effective 'territorial' protection is reflected in an effective species protection.

The anthropogenic influence on the landscape of the study region was assessed using two separate analyses. These two studies focussed on the relationship between species richness and the degree of landscape fragmentation on one hand and landscape modification on the other hand.

The degree of landscape fragmentation was assessed by applying the Effective Mesh Size Index $\left(m_{e f f}\right)$. The relationship between $m_{e f f}$ and species richness of the total number of species as well as five sub-groups of vascular plants aggregated using their residence and threat status was assessed using a variation partitioning approach that separated the effects of landscape fragmentation, environmental conditions and spatial autocorrelation. Landscape fragmentation was found to significantly affect richness of all species groups. However, the strength of the effect varied between species, being largest for neophytes and smallest for threatened species.

A clustering analysis on landscape metrics describing landscape composition and configuration revealed a six-level gradient of landscape modification for the study region. The gradient spanned from highly fragmented urban landscapes to little fragmented landscapes with a high proportion of forest. Species richness of the total number of species as well as seven sub-groups of vascular plants aggregated using the residence and threat status as well as habitat preferences of the species were found to significantly differ along the gradient. For the entire and native species pool as well as for forest, low-nutrient indicating and threatened plants, species numbers peaked at the opposite ends of the modification gradient. For neophytes, urban plants and highnutrient indicators richness decreased along the gradient from high species numbers in urbanised to low numbers in less modified landscapes.

The results obtained from the investigations in this thesis provide a basis for future landscape and conservation planning approaches in the study region by facilitating the identification of landscapes of high conservation value and/or concern. Provided that appropriate, high quality data like those used in the present thesis are available, the methodologies applied and developed in context of the thesis can be transferred to other regions to accordingly support conservation and landscape planning. 


\section{Zusammenfassung}

Die auf verschiedenen Skalen-Ebenen heterogene Verteilung des Artenreichtums fasziniert Naturforscher und Biologen seit Jahrhunderten, so dass eine Vielzahl von Hypothesen zur Erklärung dieser Muster aufgestellt wurde. Es besteht breiter Konsens darüber, dass eine Vielzahl verschiedener Prozesse für die Variation des Artenreichtums verantwortlich ist. Je nachdem welche Taxa, welche Orte und welche räumlichen und zeitlichen Skalen betrachtet werden, wird den verschiedenen Faktoren dabei eine unterschiedlich große Bedeutung zugemessen. Umweltgradienten gelten in diesem Zusammenhang als die wichtigsten, den Artenreichtum bestimmende Faktoren. Da jedoch die Landschaften und damit Habitate der Arten weltweit und vor allem in Mitteleuropa stark anthropogen überprägt sind, sollte der auf den Artenreichtum wirkende Einfluss anthropogener Interventionen in diesem Zusammenhang nicht vernachlässigt werden. Die durch den Menschen und seine Aktivitäten verursachten Landnutzungsänderungen, eine steigende Landschaftsfragmentierung und -degradation sowie die Intensivierung der Landnutzung haben sich bereits im Verlust (halb-)natürlicher Landschaftselemente, dem Rückgang der Biodiversität und der Verschlechterung von Ökosystemfunktionen niedergeschlagen; eine weitere Verschlechterung der Zustände wird vorhergesagt.

In diesem Zusammenhang dient die Ausweisung von Schutzgebieten vielfach dazu, den auf die Arten und ihre Habitate wirkenden Druck zu vermindern. Da die Lage dieser Gebiete jedoch häufig nicht auf Grundlage der Kenntnis über das Vorkommen von Arten bestimmt wurde, dürfte die Effektivität der Schutzgebiete für den Artenschutz vielfach eingeschränkt sein.

Die vorliegende, die norddeutschen Bundesländer Niedersachsen und Bremen umfassende Arbeit untersucht 1.) die Muster des Artenreichtums der Gefäßpflanzenarten in den beiden Ländern; 2.) die Effektivität des Schutzgebietsnetzwerks des norddeutschen Tieflands für den Schutz seltener und bedrohter Gefäßpflanzenarten und 3.) den Zusammenhang zwischen Mustern des Artenreichtums und den ihnen zugrundeliegenden Prozessen unter besonderer Berücksichtigung des anthropogenen Einflusses. Die Arbeit basiert auf einem umfangreichen, im Rahmen des Niedersächsischen Pflanzenartenerfassungsprogramms (NLWKN 1982-2003) erhobenen Datensatzes, der räumlich explizite Informationen zur Verbreitung aller in den Bundesländern vorkommenden Arten liefert. Die floristischen Daten wurden für die durchgeführten Analysen mit hochaufgelösten Daten zu Landbedeckung und Umweltbedingungen kombiniert. Damit repräsentiert die vorliegende Arbeit die erste umfassende Auswertung des umfangreichen floristischen Datensatzes.

Für die Untersuchung der Muster des Gefäßpflanzenreichtums innerhalb der Untersuchungsregion wurden für die Gesamtzahl der Arten sowie für fünf auf Grundla- 
ge des floristischen Status' und des Gefährdungsgrades der Arten zusammengestellte Untergruppen der Artenreichtum pro Landschaftseinheit (1.762 MesstischblattQuadranten à ca. $30 \mathrm{~km}^{2}$ ) berechnet. Der Artenreichtum aller Gruppen zeigte eine heterogene Verteilung in der Untersuchungsregion, wobei eine Zunahme der Artenzahlen von Nord nach Süd und zum Teil auch von West nach Ost erkennbar war. Die Zentren hoher Artenvielfalt der verschiedenen Gruppen korrelierten miteinander.

Die Analyse der Effektivität der Schutzgebiete für den Schutz der seltenen und bedrohten Gefäßpflanzenarten zeigte, dass die Artvorkommen im niedersächsischen Tiefland zu einem relativ hohen Anteil durch Naturschutz- und Fauna-Flora-HabitatGebiete abgedeckt sind, wobei letztere Gebiete die erstgenannten sinnvoll ergänzten. Im Rahmen der Analyse konnte jedoch nicht untersucht werden, inwiefern sich der nachgewiesene effektive Gebietsschutz in einem effektiven Artenschutz niederschlägt.

Der anthropogene Einfluss auf die Landschaft der Untersuchungsregion wurde im Rahmen zweier separater Analysen untersucht, wobei der Zusammenhang zwischen Artenreichtum und Landschaftsfragmentierung einerseits und dem Grad der Landschaftsmodifikation andererseits analysiert wurde.

Die Landschaftsfragmentierung wurde in der vorliegenden Arbeit mittels des Landschaftsmaßes der 'Effektiven Maschenweite' ( $m_{\text {eff }}$; engl. Effective Mesh Size Index) untersucht. Der Zusammenhang zwischen $m_{e f f}$ und dem Artenreichtum aller Gefäßpflanzenarten sowie fünf weiteren nach ihrem floristischen bzw. Gefährdungsstatus definierten Gruppen wurde mittels einer Varianzpartitionierung ermittelt, die die Effekte der Landschaftsfragmentierung von den durch Umweltvariablen und räumliche Autokorrelation der Daten verursachten Effekten separiert. Der Grad der Landschaftsfragmentierung erklärte einen signifikanten, jedoch unterschiedlich großen Anteil des Artenreichtums aller untersuchten Gruppen, wobei der stärkste Effekt für die Gruppe der Neophyten und der geringste für die Gruppe der seltenen und bedrohten Arten erkennbar waren.

Eine Cluster-Analyse auf Grundlage verschiedener, die Komposition und Konfiguration der Landschaft beschreibender Landschaftsmaße identifizierte für die Modellregion einen sechsstufigen 'Landschaftsmodifikations'-Gradienten. Dieser Gradient reichte von stark fragmentierten urbanen hin zu wenig fragmentierten Landschaften mit hohem Waldanteil. Der Gesamtartenreichtum sowie die Artenzahlen von sieben verschiedenen, nach ihrem floristischen Status, ihrer Gefährdung bzw. ihrer HabitatBindung bestimmten Artengruppen zeigten signifikante Unterschiede entlang des Gradienten. Die Gesamtartenzahl wie auch der Reichtum der indigenen, der bedrohten sowie der an Waldlebensräume und nährstoffarme Habitate gebundenen Arten war jeweils an den Enden des Gradienten am höchsten. Der Reichtum der Neophyten sowie der an urbane Räume und nährstoffreiche Habitate gebundenen Arten nahm dagegen von den urbanen zu den wenig beeinflussten Landschaften hin ab.

Die im Rahmen der Arbeit erhaltenen Ergebnisse können zukünftig als Grundlage für naturschutzfachliche Planungen in der Untersuchungsregion dienen, indem sie 
die Ausweisung von für den Naturschutz relevante Landschaftsräume unterstützen. Sofern entsprechende, qualitativ mit denen der vorliegenden Arbeit vergleichbare Grundlagendaten vorliegen, eignen sich die im Rahmen der Arbeit angewendeten und entwickelten Methoden, um auf andere Regionen übertragen $\mathrm{zu}$ werden und auch dort naturschutzfachliche und landschaftsplanerische Prozesse zu unterstützen. 

4 Introduction 


\subsection{Patterns of species richness at the landscape scale}

The heterogeneous geographic distribution of species richness has fascinated biologists for centuries (RICKLEFS, 2004; see BARTHLOTT et al., 2005 for an overview of the history of the exploration of diversity patterns of vascular plants). The acceleration of extinction rates in recent decades (HodApp et al., 2014) has meant that the identification and analysis of the spatial patterns of species richness is now more important than ever. Species richness is frequently referred to as an indicator for biodiversity (HodApr et al., 2014) as it is comparatively easy to measure and does not depend on knowledge of the abundance of species. Biodiversity, in turn, is widely accepted as to play an important role for ecosystem functioning (SECRETARIAT OF THE CBD, 2006; CARdinale et al., 2012; Hodapp et al., 2014). Therefore, species diversity has been used as a proxy for the ecological status of ecosystems (Hodapp et al., 2014).

The importance of biodiversity is widely acknowledged in science as well as in political decision making and therefore numerous measures to explore and protect it have been implemented. On the global scale, the Convention on Biological Diversity (Conference of the Parties, 1992) is ratified by 194 countries (status September 2014; Secretariat of THE CBD, 2014) and aims at the "conservation of biological diversity, the sustainable use of its components and the fair and equitable sharing of the benefits arising out of the utilization of genetic resources" (SECRETARIAT OF THE CBD, 2005, p. 5). These goals have been implemented in European legislation by the EU Biodiversity Strategy (European Commission, 1998). In this context, the Habitats Directive (together with the Birds Directive) forms the "cornerstone of Europe's nature conservation policy" (EUROPEAN COMMIssion, 2014) and aims at the protection of biodiversity, i. e. the diversity in flora, fauna and habitats.

As only what is known can be effectively protected, the identification of species richness patterns is an important aspect of their effective protection. Plants are seen as the "key structural elements of terrestrial ecosystems" (KREFT \& JETZ, 2007, p. 5925) as they play an important functional role as primary producers and provide habitats for animal species (CARDinale et al., 2011; SCHETter et al., 2013). Because of this, as well as the fact that data on plant species occurrences are relatively easy to collect compared to the collection of data of mobile organisms (SCHETTER et al., 2013), a lot of research has focused on these organisms. Patterns of vascular plant species richness have been investigated on the global (Myers et al., 2000; BARTHLOtT et al., 2005; KiER et al., 2005) as well as on the European scale (EssL et al., 2013) and have been used for global-scale conservation priority setting (MYERs et al., 2000).

Species richness patterns may be either directly derived by summarising the knowledge on the spatial distribution of single species (see e.g. Haeupler, 2000; Garve, 2007) or - if reliable data are missing - estimated by applying modelling approaches (cp. Kier et al., 2005; MAes et al., 2005; Kreft \& Jetz, 2007).

The availability of digital data on species occurrences on a regional and national level has increased rapidly in recent years. At the global scale, the Global Biodiver- 
sity Information Facility (GBIF) provides access to more than 400 million spatially explicit records of several taxonomic groups, including vascular plants (GBIF, 2014). In Europe, most countries maintain species mapping projects that gather data on the distribution and presence or even abundance of plant and animal species (see e.g. Zajac \& Zajac, 2001; Landuyt et al., 2012; NetPhyD \& BfN, 2013). The mapping of species occurrences in Germany is supervised by the federal states (see e. g. HARDTKE \& IHL, 2000; Korsch et al., 2002; Haeupler et al., 2003). The results of this mapping are then reported to the federal authority.

Data on plant species occurrences provide a good basis for conservation planning (BRоокs et al., 2004). Therefore information obtained from floristic mapping have been applied for various purposes. For example, HAeupler \& Vogel (1999) used data obtained from such a mapping to derive patterns of species richness for the whole of Germany. Based on species number of rare and threatened plants per landscape unit, the authors determined eight 'hotspots' ${ }^{1}$ of phytodiversity distributed across the country. On a smaller scale, WINTER et al. (2010b) used information on the occurrences of threatened plant species in an administrative district of Germany to identify Important Plant Areas (IPAs) that form an integral part of the Global Strategy for Plant Conservation (The Secretariat of the CBD, 2002).

Species records have furthermore been used to analyse the indicative power of selected sub-groups of plant species for the overall species richness of a region: For north-west Germany, DiekMANN et al. (2008) used records of forest species taken from a flora atlas of the Weser-Elbe region and related the richness of these species to that of the overall number of plants reported for the region. The authors showed that species numbers of this group are indicative for total species richness as well as for the number of threatened species. Similarly, Kati et al. (2004) found that woody plants acted as suitable indicators for the richness of other taxonomic groups in the Mediterranean.

In the study area of the present thesis, the federal states of Lower Saxony and Bremen, species records amongst other things serve as a basis for the creation of distribution maps ${ }^{2}$, the compilation of Red Lists and as a contribution to landscape and environmental planning (ScHupp et al., 2001).

\subsection{Linking patterns and processes: What influences species richness patterns at the landscape scale?}

The analysis of the underlying causes of species richness patterns is a major goal in ecological, evolutionary, and biogeographical research (GASTON, 2000; GODFRAY \&

\footnotetext{
${ }^{1}$ In the following the term 'hotspot' is used for landscape units hosting a high richness of threatened plant species (cp. definition of HAEUPLER \& VOGEL, 1999).

${ }^{2}$ See Garve (1994) for such maps for threatened plant species and Garve (2007) for all vascular plant species of the state.
} 
LAWTON, 2001; Gotelli et al., 2009; Wiens, 2011) and has challenged researchers for centuries (RICKLEFS, 2004). In recent decades, researchers have provided many different ideas on the question of what influences richness patterns (see RICKLEFS, 2004 for an overview on the history of these concepts). In accordance with many researchers, Colwell et al. (2004, p. E14) suggests that "multiple factors are surely at work and that any particular factor may be more or less important for different taxa, different places, and different spatial and temporal scales". Processes and factors influencing species richness patterns include speciation and extinction rates (COLWELl et al., 2004; WIENS, 2011), geometric constraints (i. e. mid-domain effect, MDE, Colwell \& HURTT, 1994) as well as geographic patterns of temperature, productivity, potential evapotranspiration, topography, formation history (Colwell et al., 2004) and human activities (WhIte \& KeRR, 2007; WANG et al., 2011). The following two subsections will focus on the role of abiotic environmental conditions and the human influence shaping species richness patterns. It has to be kept in mind, though, that the underlying processes influencing these patterns are far too complex to be fully disentangled. Thus, usually the processes are approached by using indicators or surrogates.

\subsubsection{Environmental conditions as key drivers for richness patterns of plants}

The role of the abiotic environment in shaping species richness patterns has been analysed on various spatial scales ranging from global (KREFT \& JETZ, 2007) to local assessments (HeIKKINEN, 1996). BARTHLOTT et al. (2007, p. 308) describes 'geodiversity', i. e. "the heterogeneity of topography, geology, soils, or climate", as the main driver for global spatial patterns in species richness. More specifically, KREFT \& JeTz (2007) showed that for vascular plants potential evapotranspiration, the number of wet days per year, and measurements of topographical and habitat heterogeneity are core predictors of global richness patterns. Similar factors were found to explain species richness on a smaller scale: Elevation range followed by minimum monthly potential evapotranspiration was identified as to be the most important factor determining woody plant species richness of the Iberian Peninsula VetaAs \& Ferrer-Castán (2008). Similarly, climatic variables like temperature and potential evapotranspiration were found to be by far the most important predictors for vascular plant species richness in Austria (Moser et al., 2005). For alien plant species richness in the European Alps, environmental heterogeneity proved to be the best surrogate (DAINESE et al., 2014). The same factor in combination with the presence of calcareous rock outcrops and threshold values of minimum precipitation was found to also determine species richness in Switzerland (WoHLGEMUTH, 1998). For Germany, native and alien plant species richness was found to be largely dependent on the diversity of geological substrates (KüHN et al., 2003). Similarly, on the local scale the overall habitat diversity was identified as the main driver for species richness of vascular plants in a nature reserve in northern Finland (HEIKKINEN, 1996). 
All these findings promote the hypothesis that overall species richness tends to be higher in a varied environment compared to a monotonous one, as heterogeneous environmental conditions provide a greater diversity of niches potentially suitable for plant species (Honnay et al., 2003; AdAMs, 2009).

\subsubsection{The human impact on richness patterns of plants}

The previous subsection dealt with the role of the abiotic environment in shaping species richness patterns. Undoubtedly, environmental conditions are the main drivers underlying species richness patterns on a large scale. However, as many landscapes and thus species' habitats worldwide and particularly in Central Europe are strongly influenced by humans (KowARIK, 1990), the shaping effect of human interventions on richness patterns should not be overlooked.

Over the last few decades, much attention has been focused on the analysis of the effects of human interventions at the landscape scale. In this context, a large number of studies have searched for surrogates describing the human impact on landscapes and biodiversity.

\section{Human-induced threats to and protection of plant species richness}

Habitat loss, fragmentation and degradation have been identified as being the main factors threatening species richness (FAHRIG, 2001; AgUILAR et al., 2006; KRAUss et al., 2010). Both habitat fragmentation and loss - the latter being an "important by-product of habitat fragmentation" (McGARIGAL, 2014, p. 89) - are known to increase the isolation between plant or animal sub-populations, thereby impeding the genetic exchange between individuals. The resulting loss in genetic variation may increase the probability of extinction by demographic and environmental stochasticity (Boor et al., 2000). In many cases, habitats are not immediately fully destroyed but undergo a gradual degradation that, however, ultimately leads to their complete destruction.

A multitude of landscape-level processes are responsible for habitat loss, fragmentation and degradation in Western Europe, including - but not being limited to - urbanisation, agricultural intensification, eutrophication and climate change (cp. Fig. 1.1).

Urbanisation $^{3}$ has been identified as one of the leading causes of species extinction (McKinney, 2006; Aronson et al., 2014). Urbanisation affects biodiversity in many ways, e. g. through the fragmentation and destruction or degradation of species' habitats and by altering the precipitation and temperature regime as well as air, soil and water quality (SuKopp \& STARFINGER, 1999). In their review on causes of species endangerment in the United States, CzECH et al. (2000) found that urbanisation in the country endangers more species than any other human activity although barely $5 \%$

3 i.e. "the process by which towns and cities are formed and become larger as more and more people begin living and working in central areas" (Definition taken from MERRIAM-WEBSTER (2014). 
of the total surface area of the United States is covered by urban and other built-up areas (MCKINNEY, 2002). In Germany, urban and traffic areas cover about $14 \%$ of the landscape (StATistische ÄMTER Des Bundes UND DER LÄNDER, 2012), and this proportion is increasing: Each day about 80 ha of undeveloped land are transferred to building land (UBA, 2014) resulting in an enormous loss of unsealed surface area and a fragmentation and loss in species' habitats. Despite the destructive effect of urbanisation on native habitats, cities are found to be generally richer in plant species than surrounding areas (HonNAY et al., 2003; WANIA et al., 2006; LIPPE \& KowARIK, 2008). This pattern might arise from different aspects: Generally, niche availability in urban areas is known to be rather high (Deutschewitz et al., 2003; Honnay et al., 2003; WANIA et al., 2006). KüHN et al. (2004) furthermore suggested that urban areas are commonly situated in naturally diverse ecoregions resulting in an overall increased species diversity. Also, alien species are known to show increased numbers in urban areas, adding to the urban species pool (Roy et al., 1999; KüHN et al., 2004).

Agricultural intensification has been proposed as one further main cause of biodiversity loss (TILman et al., 2001; TscharntKe et al., 2005). In Central Europe it accelerated in course of industrialisation in the late 19th and early 20th century (TsCHARNTKE et al., 2005; Krause \& CUlmSEe, 2013) and has been reinforced by the advent of the common agricultural policy of the EU since the 1950s (STOATE et al., 2001; KRAUSE et al., 2011). Conventional farming practices affect species diversity mainly through the conversion of non-arable land that increases habitat loss and fragmentation (TsCHARNTKE et al., 2005). However, not only the conversion of non-arable to high-productive cropland but also the abandonment of marginal sites, the removal of small-scale landscape elements and the application of pesticides and fertilisers have an effect on species (Tilman et al., 2001). Studies on the landscape scale in Europe have identified large losses in plants, invertebrates and farmland birds following agricultural intensification (ANDreasen et al., 1996; DonAld, 1998; Donald et al., 2001; ButLer et al., 2010; Storkey et al., 2012; MeYER et al., 2013).

The eutrophication of ecosystems, i.e. primarily the input of nitrogen, phosphorous and sulphur compounds, has been identified to reduce plant species richness in a broad range of European ecosystems (DISE, 2011). As high fertiliser inputs largely add to the eutrophication of a landscape, the process is tightly linked to that of agricultural intensification. Additional inputs adding to the eutrophication of ecosystems come from industry and transport (UNEP, 2014). Due to strict regulations in Europe (see 'Convention on Long-range Transboundary Air Pollution' and its eight subsequent protocols; UNECE, 1979), emissions of air pollutants, especially sulphur, have been reduced in recent years (DisE, 2011). However, critical loads are still exceeded in many semi-natural ecosystems in Europe (Hettelingh et al., 2008; Dise, 2011). Eutrophication affects species mainly through the degradation of their habitats - in the worst case habitats will become uninhabitable for the species under consideration which results in habitat loss. The negative impacts of eutrophication on species 
richness have been reported for all kinds of habitats across Europe, e. g. for acid grasslands in the Atlantic biogeographic region (STEvens et al., 2010), southern Swedish temperate oak forests (BRUNET et al., 1998), Swiss mountain grasslands (RоTH et al., 2013) and British heathlands as well as acid and calcareous grasslands (MASKELL et al., 2010).

Based on different scenarios, the impact of climate change on the distributions of plant and animal species has been frequently modelled in recent years (e. g. BAKKENES et al., 2002; Thuiller et al., 2005; Pompe et al., 2008). These analyses have shown that many European plant species are at risk from climate change (THUILLER et al., 2005). Thomas et al. (2004) predicted that due to climate change 6-8\% of all plant species in Europe could become extinct by 2050. For Germany, Pompe et al. (2008) suggested that - even under moderate climate change assumptions - negative impacts on the German flora will arise. The authors point out that due to climate change severe shifts in the species pools will occur.

Climate change scenarios up to the year 2080 for Germany have identified a definite warming trend of $1.6-3.8^{\circ} \mathrm{C}$ and small overall changes in annual precipitation $(<10 \%)$ (ZeBisch et al., 2005). However, changes in seasonal precipitation will occur with an increase in winter and a decrease in summer precipitation (ZEBIsCH et al., 2005). Due to their particular environmental characteristics, different regions in Germany are assumed to be affected by climate change by a varying degree (ZEBISCH et al., 2005). Highest vulnerability to climate change is expected for south-west and the central parts of eastern Germany as well as the Alps. The low mountain ranges of Germany as well as the coastal regions show medium vulnerability whereas northwest Germany was identified to be least vulnerable (Zевіsсн et al., 2005). Therefore, impacts of climate change on the study area of this thesis, the federal states of Lower Saxony and Bremen, can be assumed to be small compared to other German states.

The processes discussed in the previous paragraphs all add to the modification of a landscape and by doing so pose a threat to species. As it is difficult, if not impossible, to consider all of these processes separately as their effects on biodiversity in a landscape are usually interlinked, multi-layered concepts combining them have been proposed by several researchers. These concepts aim at describing the degree of modification a landscape has undergone. Concepts proposed involve 'landscape modification' (FORMAN \& Godron, 1986) and the related concepts of 'landscape naturalness' (MACHADO, 2004; LIIRA \& SEPP, 2009; WinTER et al., 2010a), 'hemeroby' (SuKopp, 1976; KoWARIK, 1988; GrabHerr et al., 1998; SteINHARdt et al., 1999; WrbKa et al., 2004; WAlz \& SteIn, 2014), 'urbanity' (Hill et al., 2002; WrBKA et al., 2004) and the 'degree of culturalness' (JANSEN et al., 2009). These concepts are used to assess the conservation value of landscapes (WRBKA et al., 2004) and have been proposed to allow conclusions on biodiversity (WALz \& STEIN, 2014). However, until now no study has directly analysed the relationship between any of these concepts and species richness/diversity. 


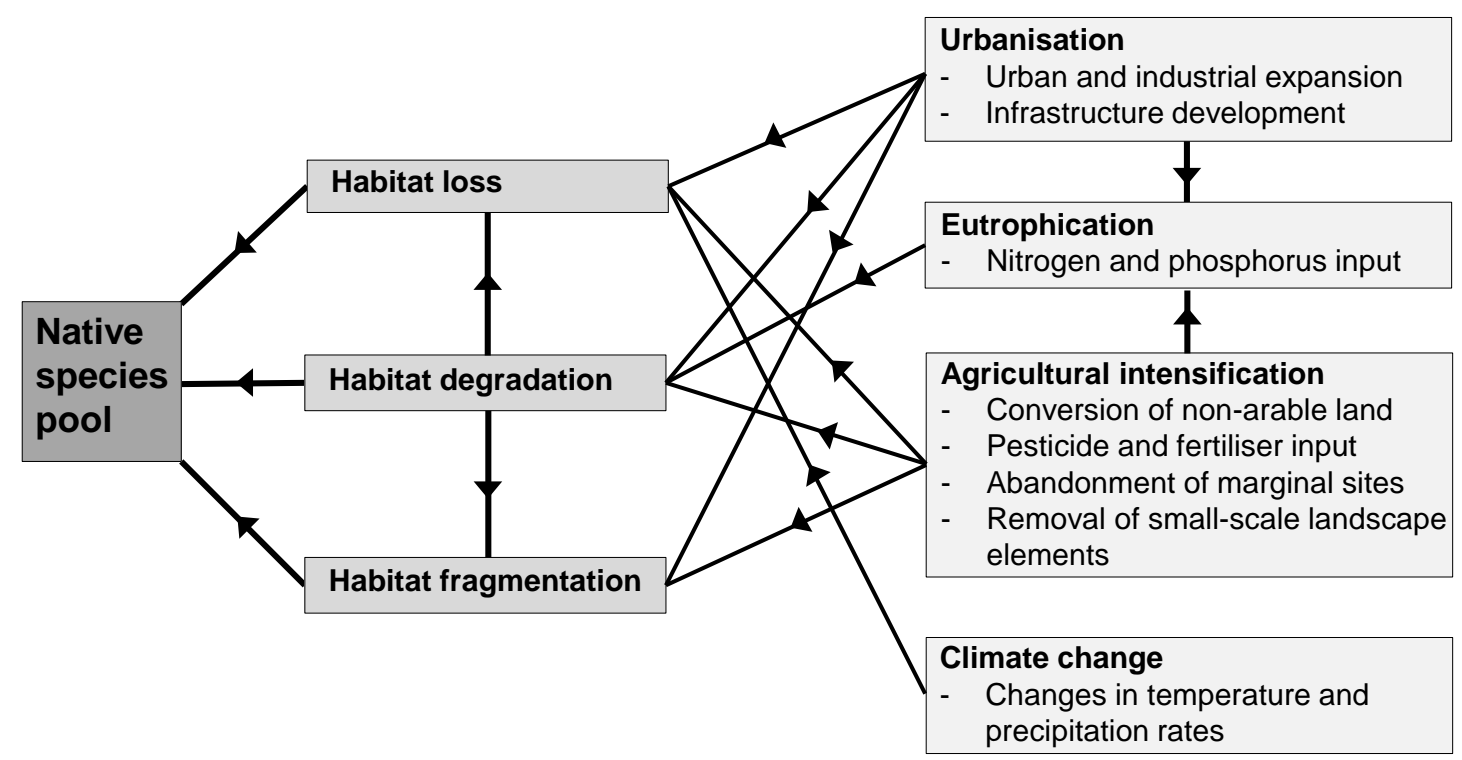

Figure 1.1: Human-induced threats to the local species pool (selection of main threats). Arrows indicate the main direction of the impact.

As discussed in the previous paragraphs, species in today's highly industrialised landscapes are threatened by a multitude of processes, most of them being linked to human-induced changes in the landscape. To reduce the negative impacts of these changes on species, a number of different concepts for the protection of species have been proposed that can generally be allocated into segregative and integrative measures (Plachter, 1991). These concepts have been developed for agricultural landscapes but can analogously be transferred to forests, water bodies and urban areas (Plachter, 1991). In segregative concepts, areas used for production are separated from those designated for the protection of species, habitats and ecosystems (JESSEL, 2012). In contrast, integrative concepts try to include both production and conservation in the same areas. Integrative measures reduce negative impacts on species by a reduction in the intensity of land use and the establishment of biotope networks (Plachter, 1991). This management can, amongst others, promote species tolerant to land use (TsCHARNTKE et al., 2012), improve ecosystem services and reduce isolation of habitats (JEsSEL, 2012; see also "dual conservation strategy", JACKSON et al., 2009). However, it can not promote the protection of species linked to (semi-)natural ecosystems (e.g. mires, rocky outcrops). For the protection of such species the segregative concept might be useful. Segregation is mainly enforced by the designation of protected areas inside which land use is forbidden, restricted or regulated to provide habitats for species otherwise lost from a region.

In Germany, there are various categories of protected areas, of which national parks, nature reserves (German: Naturschutzgebiete) and Natura 2000 sites (EU Habitats Directive) are those covering the largest areas. Protected areas are widely ac- 
cepted as being essential for the long-term conservation of biodiversity (Evans et al., 2006). However, their effectiveness in protecting species remains largely unclear as the extent and location of many protected areas have not been defined using knowledge on species occurrences (WINTER et al., 2010b; JEDICKE, 2012). Determining the effectiveness of such areas "in representing and maintaining biodiversity is a core issue in systematic conservation planning" (GASTON et al., 2006, p. 77). Nevertheless, few studies until now have focused on this topic. Regional studies that analysed the effectiveness of protected areas have identified numerous gaps in the existing protected area network (see e.g. Scott et al., 2001 for the USA, Andelman \& Willig, 2003 for the Western Hemisphere and Deguise \& KerR, 2006 for Canada). An analysis by Rodrigues et al. (2004) uncovered such gaps on a global scale. In contrast, for Great Britain, JACKSON et al. (2009) found the current protected areas to cover the occurrences of a large majority of Red List plant species. However, despite the good overall species coverage, less than one third of the total number of occurrence records were covered. It is obvious that results obtained for one particular region cannot be directly transferred to other areas and that further research in this field is strongly needed.

\section{Surrogates for the human influence on species richness patterns}

As mentioned earlier, much attention has focused on the analysis of the effects of human interventions on species richness patterns in recent decades. Studies on this topic usually have searched for surrogates describing and quantifying the human impact on landscapes and biodiversity. For this purpose, land cover data that reflect the influence of humans on the landscape as well as landscape metrics used to quantify the magnitude of this impact have been widely applied. Using landscape metrics either the composition (i.e. the abundance of patch types in a landscape) or configuration (i. e. the spatial arrangement of patches) of a landscape can be assessed (cp. subsection 2.2.5). Indices used include measures of landscape diversity, patch shape complexity, edge length and density, core area sizes as well as metrics used to quantify landscape fragmentation, landscape/habitat connectivity and isolation. Below, some of these measures and their relevance for species richness and diversity are discussed.

Landscape diversity - analogical to 'geodiversity' (cp. subsection 1.2.1) - was identified to be a convenient surrogate for species richness (Luoto, 2000; HonNAY et al., 2003). Different measures have been used to express landscape diversity, the simplest merely reflecting the number (i.e. patch richness) or proportions of different land cover types (Deutschewitz et al., 2003; KüHN et al., 2003; Wania et al., 2006), the more complex ones combining richness and evenness of land cover types (e. g. Simpson's and Shannon's Diversity Index; see e.g. Deutschewitz et al., 2003; Honnay et al., 2003; KumAr et al., 2006). 
Several studies have analysed the relationship between species richness and landscape diversity. For Germany, a high structural and habitat diversity in combination with moderate levels of natural and/or anthropogenic disturbance was found to increase richness of native and alien plant species (Deutschewitz et al., 2003). Similarly, regional plant species richness of Flanders, Belgium was found to be positively correlated with landscape diversity expressed in terms of high patch richness and evenness and irregular patch shapes (HonNAY et al., 2003). As landscape diversity in the study area was found to go along with the degree of urbanisation also a positive correlation between the percentage of built-up area and species richness was stated. As previously discussed in the paragraph on the effects of urbanisation, the latter has been frequently reported by researchers and has been attributed to the increased niche availability in urban areas.

However, not only landscape diversity measures have been applied to explain species richness patterns. Several studies have used patch shape complexity as a surrogate for species richness as they assumed that simple patch shapes will be dominant in landscapes strongly influenced by humans, whereas more natural landscapes should be characterised by irregular shaped patches (MOSER et al., 2002; YAMAURA et al., 2008). In the study of Moser et al. (2002) an index of patch shape complexity developed by the authors that is based on the number of shape-characterising points along a polygon's boundary proved to be a good predictor for the species richness of vascular plants and bryophytes in Austria: the authors found species richness to be highest in landscapes for which irregular patch shapes were reported. Similarly, for a Mediterranean forest landscape SCHINDLER et al. (2013) identified patch shape to be a good indicator for the diversity of woody plants. In contrast, by using a shape index independent of area for Japan, the study of YAMAuRA et al. (2008) found the richness of forest-floor plants to be influenced by patch shape to only a limited extent whereas local forest structure had a much larger effect.

As landscape fragmentation resulting in habitat loss and isolation is supposed to be one of the largest threats to biodiversity worldwide (JAEGER, 2000; see also previous section on threats to species) its indicative power for species richness has been analysed by numerous studies at the local and landscape scale (see for an overview Debinski \& Holt, 2000; FAHRIG, 2003). The simplest way in which the fragmentation of a landscape can be expressed is by using measures of patch numbers or patch density (McGarigal, 2014). However, as these simple fragmentation measures are supposed to be insensitive and inconsistent in their behaviour (McGARIGAL, 2014), attempts have been made to develop alternative and more explicit measures that "behave in a consistent and logical manner across all phases of the fragmentation process" (McGarigal, 2014, p. 131). Still, despite their qualities, these indices (i.e. Landscape Division Index, Splitting Index and Effective Mesh Size; JAEger, 2000) until now have not been applied as potential surrogates for species richness. Instead, frequently, the size and the isolation of patches or the connectivity between them were used as (inverse) 
measure of fragmentation (e.g. HeLm et al., 2006; Cousins et al., 2007; Kolb, 2008; ZimbREs et al., 2013). RAATIKAINEN et al. (2009) for extensive grasslands in southern Finland used a connectivity index taking into account the distances to all possible source populations ('IFM measure', cp. MoILANEN \& NiEMINEN, 2002) to analyse how richness of spring ephemerals is related to habitat area and connectivity. They found the number of ephemerals to be highest in larger and well-connected study patches. In the study of ADRIAENS et al. (2006) a similar measure was applied to analyse how vascular plant species richness of calcareous grasslands in southern Belgium is influenced by landscape configuration. The authors found that the connectivity between grassland patches only affected the number of specialist species but not that of generalists. The opposite result was obtained by CRIstofoli et al. (2010) for heathlands in the high Ardenne (Belgium): By using measures of patch connectivity and isolation the authors found generalist, not specialist species richness to be correlated with connectivity. Interestingly, for islands in a large lake in the south-east of China, island isolation measured as the Euclidean distance to the mainland did not significantly explain vascular plant species richness (Hu et al., 2011).

The aforementioned examples show that effects of landscape fragmentation and habitat isolation may vary depending on the species groups and habitats considered (Ewers \& Didham, 2006; Rodriguez-Loinaz et al., 2012). Therefore, further studies on this important topic using different measures of fragmentation and isolation/connectivity should be undertaken for different landscapes and habitats.

\subsection{General objectives and thesis outline}

The present thesis applies spatially explicit data on the distribution of vascular plant species in combination with various information on land cover and environmental conditions to identify processes shaping species richness patterns. In doing so, the analyses focus on the significance of the anthropogenic influence and only marginally consider the importance of environmental conditions. All analyses were conducted for the federal states of Lower Saxony and Bremen, northern Germany, for which spatially explicit area-wide data on the occurrences of all vascular plant species (plant survey of Lower Saxony, NLWKN 1993-2003) as well as information on land cover and environmental conditions are available.

The main goals of the thesis are:

a) To identify and describe the patterns of vascular plant species richness in Lower Saxony and Bremen, Germany.

b) To evaluate the adequacy of the protected area network for the protection of rare and threatened plant species in the lowland region of the study area.

c) To analyse the relationship between richness patterns and the human influence on the landscape. 
The thesis is subdivided into four main data chapters (chapters 3 to 6) briefly presented in the following paragraphs. These chapters are preluded by a chapter that introduces the reader to the study area and gives an overview on the data used in the analyses (chapter 2). The thesis closes with chapter 7 which summarises and synthesises the main findings of the previous chapters and highlights open questions and future research needs.

As the key conservation work and data collection is conducted by local authorities and the large number of volunteers working for the plant survey of Lower Saxony, considerable parts of the thesis (Chapter 3 and 4) were published in German to make the results accessible to these people in charge, of which not all are familiar with the English language. This audience-focused approach guarantees that the published research can be directly implemented within the study area. The German articles are preluded by detailed English summaries to make the key results available to the global research community.

Patterns of vascular plant species richness As pointed out in section 1.1, the identification of spatial patterns of species richness provides an important key aspect of conservation planning. The study presented in chapter 3 of this thesis makes use of the extensive species record database of the vascular plant survey of Lower Saxony and Bremen to derive richness patterns of six different species groups. The groups considered in the analysis comprise (a) the total number of species that has been reported for Lower Saxony and Bremen, (b) species grouped by their residence status (native species, archaeophytes, neophytes) and (c) species grouped by their threat status (non-threatened species, threatened species). The study serves as a starting point on which the studies presented in the following chapters are based.

The following research questions are answered by interpreting maps of the richness patterns of the aforementioned species groups and by taking into account the results obtained from a correlation analysis:

- Which patterns of vascular plant species richness are observable in Lower Saxony and Bremen and the three natural landscape regions, respectively?

- Do the different plant species (sub-)groups show richness patterns that are distinguishable from each other and how much do their richness centers overlap?

- In which areas do threatened species show highest species numbers and how do these hotspots differ from that of non-threatened species?

Adequacy of the protected area network As knowledge on the spatial distribution of the rare and threatened species of Lower Saxony and Bremen is of major importance for their effective protection, occurrences of these species have been recorded at a much finer resolution (c. $2 \mathrm{~km}^{2}$ ) than those of all other species. In the study presented in chapter 4 these high-resolution data were used to test to which extent the hotspots of rare and threatened species ('RL species') in the lowlands of Lower Sax- 
ony are covered by protected areas. The analysis considers nature protection areas (Naturschutzgebiete, NSG) and NATURA 2000 sites. Additionally to the total number of threatened plant species Red List species linked to forest habitats ('RL forest species') are included in the analysis. The data are analysed using Generalised Linear Models (GLM) with Poisson distribution. The study aims at answering the following research questions:

- What patterns of diversity can be observed for the two species groups?

- Are occurrences of threatened species covered by nature protection areas?

- Does the designation of NATURA 2000 sites improve the situation?

- Are the results obtained for RL species transferable to RL forest species?

The human influence on species richness patterns As outlined in section 1.2, species richness patterns in industrialised countries are assumed to be strongly affected by human activities. To understand the magnitude and direction of this influence and the underlying regulatory processes is an important field of research. The two chapters dealing with this subject (chapter 5 and 6) use different methodological approaches to illuminate the relationship between species richness patterns and human influence.

The study presented in chapter 5 investigates whether landscape fragmentation expressed by means of the Effective Mesh Size Index is related to the richness of six different groups of vascular plants aggregated using information on their residence and threat status, respectively. The index has frequently been suggested to be ecologically meaningful (JAEGER, 2000; JAEGER et al., 2008; GIRVETZ et al., 2008) and is widely applied in studies on landscape fragmentation. However, its relevance for biodiversity has not been tested and its suitability as an indicator of biodiversity has even been questioned (EssweIN, 2007). The presented study aims to shed light on this topic by applying variation partitioning techniques used to disentangle the relationship between species richness, landscape fragmentation and environmental variables. Spatial autocorrelation in the data is considered by using PCNM analysis. It is hypothesised that:

- Landscape fragmentation, expressed as Effective Mesh Size $\left(m_{e f f}\right)$, varies in space, which results in a gradient in the degree of fragmentation within the sample of landscape units used in this study.

- The choice of fragmentation geometries that are used for the calculation of $m_{\text {eff }}$ is of major importance for explaining plant species richness patterns on the landscape scale.

- Plant species richness varies between landscape units along the fragmentation gradient, with the effect size differing between different groups of species dependent on their residence (natives, archaeophytes and neophytes) and threat status (threatened vs. non-threatened). 
The study presented in chapter 6 makes use of k-means clustering to derive a gradient of 'landscape modification' (cp. subsection 1.2.2) for 1386 landscape units of Lower Saxony and Bremen. The analysis is based on landscape metrics quantifying landscape composition and configuration. Clusters differing in terms of their degree of landscape modification are set up and placed along a 'gradient of landscape modification' according to the one proposed by Forman \& GodRON (1986). The gradient is then linked to richness patterns of the total number of vascular plant species as well as that of seven sub-groups that were aggregated using information on residence and threat status and habitat preferences of the species. The study seeks to verify the following hypothesis:

- Based on landscape metrics used to assess the composition and configuration of a landscape, clusters of landscapes with similar characteristics can be differentiated. These can be placed along a gradient of landscape modification as proposed by Forman \& GoDron (1986), spanning from (semi-)natural (mostly forested) to urbanised landscapes.

- The landscape clusters are meaningful for vascular plant species richness, i.e. species numbers vary significantly along the gradient of landscape modification intensity.

- Species numbers of different vascular plant species groups show different patterns along the gradient of landscape modification intensity. 


\section{References}

Adams, J. (2009): Species richness: Patterns in the diversity of life. Springer, Berlin, Heidelberg. 386 pp.

Adriaens, D.; Honnay, O. \& Hermy, M. (2006): No evidence of a plant extinction debt in highly fragmented calcareous grasslands in Belgium. Biological Conservation 133: $212-224$.

Aguilar, R.; Ashworth, L.; Galetto, L. \& Aizen, M. A. (2006): Plant reproductive susceptibility to habitat fragmentation: review and synthesis through a metaanalysis. Ecology Letters 9: 968-980.

Andelman, S. J. \& Willig, M. R. (2003): Present patterns and future prospects for biodiversity in the Western Hemisphere. Ecology Letters 6: 818-824.

Andreasen, C.; Stryhn, H. \& Streibig, J. C. (1996): Decline in the flora in Danish arable fields. Journal of Applied Ecology 33: 619-626.

Aronson, M. F. J.; La Sorte, F.; Nilon, C. H.; Katti, M.; Goddard, M. A.; LepczyK, C. A.; Warren, P. S.; Williams, N. S. G.; Cilliers, S.; Clarkson, B.; Dobbs, C.; Dolan, R.; Hedblom, M.; Klotz, S.; Koommans, J. L.; KüHn, I.; Macgregor-Fors, I.; McDonnell, M.; Mörtberg, U.; Pysek, P.; Siebert, S.; Sushinsky, J.; Werner, P. \& Winter, M. (2014): A global analysis of the impacts of urbanization on bird and plant diversity reveals key anthropogenic drivers. Proceedings. Biological sciences / The Royal Society 281: 20133330.

Bakkenes, M.; Alkemade, J. R. M.; Ihle, F.; Leemans, R. \& Latour, J. B. (2002): Assessing effects of forecasted climate change on the diversity and distribution of European higher plants for 2050. Global Change Biology 8: 390-407.

Barthlott, W.; Hostert, A.; Kier, G.; Koper, W.; Kreft, H.; MutKe, J.; Rafiqpoor, M. D. \& Sommer, J. H. (2007): Geographic patterns of vascular plant diversity at continental to global scales. Erdkunde 61: 305-315.

Barthlott, W.; MutKe, J.; Rafiqpoor, D.; Kier, G. \& Kreft, H. (2005): Global centers of vascular plant diversity. Nova Acta Leopoldina NF 92: 61-83.

Booy, G.; Hendriks, R. J. J.; Smulders, M. J. M.; Groenendael, J. M. v. \& Vosman, B. (2000): Genetic diversity and the survival of populations. Plant Biology 2: 379-395.

Brooks, T. M.; Da Fonseca, G. A. B. \& Rodrigues, A. S. L. (2004): Species, data, and conservation planning. Conservation Biology 18: 1682-1688.

Brunet, J.; Diekmann, M. \& Falkengren-Grerup, U. (1998): Effects of nitrogen deposition on field layer vegetation in south Swedish oak forests. Environmental Pollution 102: 35-40. 
Butler, S. J.; Boccaccio, L.; Gregory, R. D.; VoriseK, P. \& Norris, K. (2010): Quantifying the impact of land-use change to European farmland bird populations. Agriculture, Ecosystems \& Environment 137: 348-357.

Cardinale, B. J.; Duffy, J. E.; Gonzalez, A.; Hooper, D. U.; Perrings, C.; Venail, P.; Narwani, A.; Mace, G. M.; Tilman, D.; Wardle, D. A.; Kinzig, A. P.; Daily, G. C.; Loreau, M.; Grace, J. B.; Larigauderie, A.; Srivastava, D. S. \& Naeem, S. (2012): Biodiversity loss and its impact on humanity. Nature 486: 59-67.

Cardinale, B. J.; Matulich, K. L.; Hooper, D. U.; Byrnes, J. E.; Duffy, E.; Gamfeldt, L.; Balvanera, P.; O'Connor, M. I. \& Gonzalez, A. (2011): The functional role of producer diversity in ecosystems. American journal of botany 98: 572-592.

Colwell, R. K. \& Hurtt, G. C. (1994): Nonbiological gradients in species richness and a spurious Rapoport effect. The American Naturalist 144: 570.

Colwell, R. K.; RahbeK, C. \& Gotelli, N. J. (2004): The mid-domain effect and species richness patterns: What have we learned so far? The American Naturalist 163: E1-E23.

Conference of the Parties (1992): Convention on Biological Diversity (CBD). Rio de Janeiro.

Cousins, S. A. O.; Ohlson, H. \& ErIKsson, O. (2007): Effects of historical and present fragmentation on plant species diversity in semi-natural grasslands in Swedish rural landscapes. Landscape Ecology 22: 723-730.

Cristofoli, S.; Monty, A. \& MAhy, G. (2010): Historical landscape structure affects plant species richness in wet heathlands with complex landscape dynamics. Landscape and Urban Planning 98: 92-98.

Czech, B.; Krausman, P. R. \& Devers, P. K. (2000): Economic associations among causes of species endangerment in the United States. BioScience 50: 593-601.

DAINESE, M.; KüHN, I. \& Bragazza, L. (2014): Alien plant species distribution in the European Alps: influence of species' climatic requirements. Biological Invasions 16: 815-831.

Debinski, D. M. \& Holt, R. D. (2000): A survey and overview of habitat fragmentation experiments. Conservation Biology 14: 342-355.

Deguise, I. E. \& Kerr, J. T. (2006): Protected areas and prospects for endangered species conservation in Canada. Conservation Biology 20: 48-55.

Deutschewitz, K.; Lausch, A.; KüHN, I. \& Klotz, S. (2003): Native and alien plant species richness in relation to spatial heterogeneity on a regional scale in Germany. Global Ecology and Biogeography 12: 299-311. 
Diekmann, M.; Dupré, C.; Kolb, A. \& Metzing, D. (2008): Forest vascular plants as indicators of plant species richness: A data analysis of a flora atlas from northwestern Germany. Plant Biosystems 142: 584-593.

Dise, N. B. (2011): Nitrogen as a threat to European terrestrial biodiversity. In: Sutton, M. A.; Howard, C. M.; Erisman, J. W.; Billen, G.; Bleeker, A.; Grennfelt, P.; van Grinsven, H. \& Grizzetti, B. (eds.) The European nitrogen assessment, Cambridge University Press, Cambridge, UK and New York. pp. 463-494.

Donald, F. R.; Green, R. E. \& Heath, F. M. (2001): Agricultural intensification and the collapse of Europe's farmland bird populations. Proceedings of the Royal Society B - Biological Sciences 268: 25-29.

Donald, P. F. (1998): Changes in the abundance of invertebrates and plants on British farmland. British Wildlife 9: 279-289.

Essl, F.; Moser, D.; Dirnböck, T.; Dullinger, S.; Milasowszky, N.; Winter, M. \& Rabitsch, W. (2013): Native, alien, endemic, threatened, and extinct species diversity in European countries. Biological Conservation 164: 90-97.

EssweIN, H. (2007): Der Landschaftszerschneidungsgrad als Indikator für Biodiversität? BfN-Skripten 207: 157-164.

European Commission (1998): Communication of the European Commission to the Council and the Parliament on a European Community Biodiversity Strategy. URL http://www.faunaeur.org/documents/ComBioDivStrat.pdf. Retrieved 09/23/2014.

European Commission (2014): The Habitats Directive. URL http://ec.europa. eu/environment/nature/legislation/habitatsdirective/index_en.htm. Retrieved $09 / 23 / 2014$.

Evans, K. L.; Rodrigues, A. S L; Chown, S. L. \& Gaston, K. J. (2006): Protected areas and regional avian species richness in South Africa. Biology letters 2: 184-188.

Ewers, R. M. \& Didham, R. K. (2006): Confounding factors in the detection of species responses to habitat fragmentation. Biological Reviews 81: 117-142.

FAHRIG, L. (2001): How much habitat is enough? Biological Conservation 100: 65-74.

FAHRIG, L. (2003): Effects of habitat fragmentation on biodiversity. Annual Review of Ecology, Evolution, and Systematics 34: 487-515.

Forman, R. T. T. \& Godron, M. (1986): Landscape ecology. John Wiley \& sons, New York. $644 \mathrm{pp}$. 
GARVE, E. (1994): Atlas der gefährdeten Farn- und Blütenpflanzen in Niedersachsen und Bremen: Kartierung 1982 - 1992. Naturschutz und Landschaftspflege in Niedersachsen 30: 1-895.

GARVE, E. (2007): Verbreitungsatlas der Farn- und Blütenpflanzen in Niedersachsen und Bremen. Naturschutz und Landschaftspflege in Niedersachsen 43: 1-507.

Gaston, K. J. (2000): Global patterns of biodiversity. Nature 405: 220-227.

Gaston, K. J.; Charman, K.; Jackson, S. F.; Armsworth, P. R.; Bonn, A.; Briers, R. A.; Callaghan, C. S. Q.; Catchrole, R.; Hopkins, J.; Kunin, W. E.; Latham, J.; Opdam, P.; Stoneman R.; Stroud, D. A. \& Tratt, R. (2006): The ecological effectiveness of protected areas: The United Kingdom. Biological Conservation 132: 76-87.

GBIF (2014): Global Biodiversity Information Facility: Free and open access to biodiversity data. URL http://www.gbif.org. Retrieved 08/20/2014.

Girvetz, E. H.; Thorne, J. H.; Berry, A. M. \& Jaeger, J. A. G. (2008): Integration of landscape fragmentation analysis into regional planning: A statewide multi-scale case study from California, USA. Landscape and Urban Planning 86: 205-218.

Godfray, H. J. \& Lawton, J. H. (2001): Scale and species numbers. Trends in Ecology $\mathcal{E}$ Evolution 16: 400-404.

Gotelli, N. J.; Anderson, M. J.; Arita, H. T.; ChaO, A.; Colwell, R. K.; Connolly, S. R.; Currie, D. J.; Dunn, R. R.; Graves, G. R.; Green, J. L.; Grytnes, J.-A.; Jiang, Y.-H.; Jetz, W.; Lyons, K. S.; McCain, C. M.; Magurran, A. E.; RahbeK, C.; Rangel, T. F. L. V. B.; Soberón, J.; Webb, C. O. \& Willig, M. R. (2009): Patterns and causes of species richness: a general simulation model for macroecology. Ecology Letters 12: 873-886.

Grabherr, G.; Koch, G.; Kirchmeir, H. \& Reiter, K. (1998): Hemerobie österreichischer Waldökosysteme, vol. 17. Universitätsverlag Wagner, Innsbruck. 493 pp.

Haeupler, H. (2000): Die ungleiche Verteilung der Artenvielfalt innerhalb Deutschlands - Eine Antwort. Floristische Rundbriefe 34: 17-24.

Haeupler, H.; Jagel. A. \& Schumacher, W. (2003): Verbreitungsatlas der Farn- und Blütenpflanzen in Nordrhein-Westfalen. Landesamt für Ökologie, Bodenordnung und Forsten NRW, Recklinghausen. 616 pp.

Haeupler, H. \& Vogel, A. (1999): Plant diversity in Germany: a second review. Acta Botanica Fennica 162: 55-59.

HardtKe, H.-J. \& IhL, A. (2000): Atlas der Farn- und Samenpflanzen Sachsens. Dresden. 806 pp. 
HeikKinen, R. K. (1996): Predicting patterns of vascular plant species richness with composite variables: A meso-scale study in Finnish Lapland. Vegetatio 126: 151-165.

Helm, A.; Hanski, I. \& Pärtel, M. (2006): Slow response of plant species richness to habitat loss and fragmentation. Ecology Letters 9: 72-77.

Hettelingh, J.-P.; Posch, M. \& Slootweg, J. (2008): Status of the critical loads database and impact assessment. In: Hettelingh, J.-P.; Posch, M. \& Slootweg, J. (eds.) Critical load, dynamic modelling and impact assessment in Europe. pp. 15-28.

Hill, M. O.; Roy, D. B. \& Thompson, K. (2002): Hemeroby, urbanity and ruderality: bioindicators of disturbance and human impact. Journal of Applied Ecology 39: 708720.

Hodapp, D.; Kraft, D. \& Hillebrand, H. (2014): Can monitoring data contribute to the biodiversity-ecosystem function debate? Evaluating data from a highly dynamic ecosystem. Biodiversity and Conservation 23: 405-419.

Honnay, O.; Piessens, K.; Landuyt, W. v.; Hermy, M. \& Gulinck, H. (2003): Satellite based land use and landscape complexity indices as predictors for regional plant species diversity. Landscape and Urban Planning 63: 241-250.

Hu, G.; Feeley, K. J.; Wu, J.; Xu, G. \& Yu, M. (2011): Determinants of plant species richness and patterns of nestedness in fragmented landscapes: evidence from landbridge islands. Landscape Ecology 26: 1405-1417.

Jackson, S. F.; WALKER, K. \& GAStON, K. J. (2009): Relationship between distributions of threatened plants and protected areas in Britain. Biological Conservation 142: 1515-1522.

JAEger, J. A. G. (2000): Landscape division, splitting index, and effective mesh size: New measures of landscape fragmentation. Landscape Ecology 15: 115-130.

Jaeger, J. A. G.; Bertiller, R.; Schwick, C.; Müller, K.; Steinmeier, C.; Ewald, K. C. \& GHazoul, J. (2008): Implementing landscape fragmentation as an indicator in the Swiss Monitoring System of Sustainable Development (MONET). Journal of Environmental Management 88: 737-751.

Jansen, F.; Zerbe, S. \& Succow, M. (2009): Changes in landscape naturalness derived from a historical land register - a case study from NE Germany. Landscape Ecology 24: 185-198.

JEDICKE, E. (2012): 20 Jahre Natura 2000 - Zeit für eine Qualitätsoffensive. Natur und Landschaft 44: 129-130. 
Jessel, B. (2012): Naturschutz zwischen Integration und Segregation - Wohin geht der Weg? Talk at 31st German day of nature conservation, Erfurt. URL http://www.deutscher-naturschutztag.de/fileadmin/user_upload/ Plenumsvortraege_PDFs/Plenumsvortrag_Jessel.pdf. Retrieved 10/15/2014.

Kati, V.; Devillers, P.; Dufrêne, M.; Legakis, A.; Vokou, D. \& Lebrun, P. (2004): Testing the value of six taxonomic groups as biodiversity indicators at a local scale. Conservation Biology 18: 667-675.

Kier, G.; Mutke, J.; Dinerstein, E.; Ricketts, T. H.; Kuper, W.; Kreft, H. \& BARThlotт, W. (2005): Global patterns of plant diversity and floristic knowledge. Journal of Biogeography 32: 1107-1116.

KolB, A. (2008): Habitat fragmentation reduces plant fitness by disturbing pollination and modifying response to herbivory. Biological Conservation 141: 2540-2549.

Korsch, H.; Westhus, W. \& ZüNDORF, H.-J. (2002): Verbreitungsatlas der Farn- und Blütenpflanzen Thüringens. Weissdorn-Verlag, Jena. 419 pp.

KowariK, I. (1988): Zum menschlichen Einfluß auf Flora und Vegetation: Theoretische Konzepte und ein Quantifizierungsansatz am Beispiel von Berlin (West). Techn. Univ., Berlin. 280 pp.

KOWARIK, I. (1990): Some responses of flora and vegetation to urbanization in Central Europe. In: Suкopp, H.; Hejný, S. \& Kowarik, I. (eds.) Urban ecology, SPB Academic Publishing, Den Hague, Netherlands. pp. 45-74.

Krause, B. \& Culmsee, H. (2013): The significance of habitat continuity and current management on the compositional and functional diversity of grasslands in the uplands of Lower Saxony, Germany. Flora 208: 299-311.

Krause, B.; Culmsee, H.; Wesche, K.; Bergmeier, E. \& Leuschner, C. (2011): Habitat loss of floodplain meadows in north Germany since the 1950s. Biodiversity and Conservation 20: 2347-2364.

Krauss, J.; Bommarco, R.; Guardiola, M.; Heikkinen, R. K.; Helm, A.; Kuussaari, M.; Lindborg, R.; Ockinger, E.; Partel, M.; Pino, J.; Poyry, J.; RaAtikainen, K. M.; Sang, A.; Stefanescu, C.; Teder, T.; Zobel, M. \& Steffan-Dewenter, I. (2010): Habitat fragmentation causes immediate and time-delayed biodiversity loss at different trophic levels. Ecology Letters 13: 597-605.

Kreft, H. \& Jetz, W. (2007): Global patterns and determinants of vascular plant diversity. Proceedings of the National Academy of Sciences of the United States of America 104: 5925-5930. 
KüHN, I.; BRANDL, R. \& Klotz, S. (2004): The flora of German cities is naturally species rich. Evolutionary Ecology Research 6: 749-764.

KüHN, I.; BrandL, R.; MaY, R. \& KLOTZ, S. (2003): Plant distribution patterns in Germany - Will aliens match natives? Feddes Repertorium 114: 559-573.

Kumar, S.; Stohlgren, T. J. \& Chong, G. W. (2006): Spatial heterogeneity influences native and nonnative plant species richness. Ecology 87: 3186-3199.

Landuyt, W. v.; VAnhecke, L. \& Brosens, D. (2012): Florabank1: a grid-based database on vascular plant distribution in the northern part of Belgium (Flanders and the Brussels Capital region). PhytoKeys 12: 59-67.

LIIRA, J. \& SEPP, T. (2009): Indicators of structural and habitat natural quality in boreo-nemoral forests along the management gradient. Annales Botanici Fennici 46: 308-325.

LIPPE, M. v. D. \& KowARIK, I. (2008): Do cities export biodiversity? Traffic as dispersal vector across urban-rural gradients. Diversity and Distributions 14: 18-25.

Luoto, M. (2000): Modelling of rare plant species richness by landscape variables in an agriculture area in Finland. Plant Ecology 149: 157-168.

Machado, A. (2004): An index of naturalness. Journal for Nature Conservation 12: 95-110.

Maes, D.; Bauwens, D.; Bruyn, L. D.; Anselin, A.; Vermeersch, G.; van Landuyt, W.; KNIJF, G. D. \& Gilbert, M. (2005): Species richness coincidence: conservation strategies based on predictive modelling. Biodiversity and Conservation 14: 13451364.

Maskell, L. C.; Smart, S. M.; Bullock, J. M.; Thompson, K. E. N. \& Stevens, C. Y. J. (2010): Nitrogen deposition causes widespread loss of species richness in British habitats. Global Change Biology 16: 671-679.

McGarigal, K. (2014): Fragstats help. URL http://www.umass.edu/landeco/ research/fragstats/documents/fragstats.help.4.2.pdf. Retrieved 03/02/2014.

McKinneY, M. L. (2002): Urbanization, biodiversity and conservation. BioScience 52: 883-890.

MCKinney, M. L. (2006): Urbanization as a major cause of biotic homogenization. Biological Conservation 127: 247-260.

MerRIAM-Webster (2014): Merriam-Webster Online: Dictionary and Thesaurus. URL http://www.merriam-webster.com. Retrieved 10/26/2014. 
Meyer, S.; Wesche, K.; Krause, B. \& Leuschner, C. (2013): Dramatic losses of specialist arable plants in Central Germany since the 1950s /60s - a cross-regional analysis. Diversity and Distributions 19: 1175-1187.

Moilanen, A. \& Nieminen, M. (2002): Simple connectivity measures in spatial ecology. Ecology 83: 1131-1145.

Moser, B.; Zechmeister, H. G.; Plutzar, C.; Sauberer, N.; Wrbka, T. \& Grabherr, G. (2002): Landscape patch shape complexity as an effective measure for plant species richness in rural landscapes. Landscape Ecology 17: 657-669.

Moser, D.; Dullinger, S.; Englisch, T.; Niklfeld, H.; Plutzar, C.; Sauberer, N.; Zechmeister, H. G. \& GrabHerR, G. (2005): Environmental determinants of vascular plant species richness in the Austrian Alps. Journal of Biogeography 32: 1117-1127.

Myers, N.; Mittermeier, R. A.; Mittermeier, C. G.; Fonseca, G. A. B. DA \& Kent, J. (2000): Biodiversity hotspots for conservation priorities. Nature 403: 853-858.

NetPhyD \& BfN (2013): Verbreitungsatlas der Farn- und Blütenpflanzen Deutschlands. Landwirtschaftsverlag Münster, Münster. 912 pp.

Plachter, H. (1991): Naturschutz, vol. 1563. Fischer, Stuttgart. 463 pp.

Pompe, S.; Hanspach, J.; Badeck, F.; Klotz, S.; Thuiller, W. \& Kühn, I. (2008): Climate and land use change impacts on plant distributions in Germany. Biology Letters 4: 564-567.

RaAtikainen, K. M.; HeikKinen, R. K. \& Luoto, M. (2009): Relative importance of habitat area, connectivity, management and local factors for vascular plants: spring ephemerals in boreal semi-natural grasslands. Biodiversity and Conservation 18: 1067-1085.

RICKLEFS, R. E. (2004): A comprehensive framework of global patterns in biodiversity. Ecology Letters 7: 1-15.

Rodrigues, A. S. L.; Andelman, S. J.; Bakarr, M. I.; Boitani, L.; Brooks, T. M.; Cowling, R. M.; Fishrool, L. D.; Da Fonseca, G. A. B.; Gaston, K. J.; Hoffmann, M.; Long, J. S.; Marquet, P. A.; Pilgrim, J. D.; Pressey, R. L.; Schipper, J.; Sechrest, W.; Stuart, S. N.; Underhill, L. G.; Waller, R. W.; Watts, M. E. \& YAN, X. (2004): Effectiveness of the global protected area network in representing species diversity. Nature 428: 640-643.

Rodriguez-Loinaz, G.; Amezaga, I. \& Onaindia, M. (2012): Does forest fragmentation affect the same way all growth-forms? Journal of Environmental Management 94: 125-131. 
Roth, T.; Kohli, L.; Rihm, B. \& Achermann, B. (2013): Nitrogen deposition is negatively related to species richness and species composition of vascular plants and bryophytes in Swiss mountain grassland. Agriculture, Ecosystems $\mathcal{E}$ Environment 178: 121-126.

Roy, D. B.; Hill, M. O. \& Rothery, P. (1999): Effects of urban land cover on the local species pool in Britain. Ecography 22: 507-515.

Schetter, T. A.; Walters, T. L. \& Root, K. V. (2013): A multi-scale spatial analysis of native and exotic plant species richness within a mixed-disturbance oak savanna landscape. Environmental Management 52: 581-594.

Schindler, S.; Wehrden, H. v.; Poirazidis, K.; WrbKa, T. \& Kati, V. (2013): Multiscale performance of landscape metrics as indicators of species richness of plants, insects and vertebrates. Ecological Indicators 31: 41-48.

Schupp, D.; Behm-Berkelmann, K.; Herrmann, T.; Pilgrim, B. \& Schacherer, A. (2001): Arten brauchen Daten - Erfassung von Tier- und Pflanzenarten in Niedersachsen. Informationsdienst Naturschutz Niedersachsen 5: 209-240.

Scott, J. M.; Davis, F. W.; McGhie, R. G.; Wright, R. G.; Groves, C. \& Estes, J. (2001): Nature reserved: Do they capture the full range of America's biological diversity? Ecological Applications 11: 999-1007.

Secretariat of THE CBD (2005): Handbook of the Convention on Biological Diversity: Including its Cartagena protocol on biosafety. URL http://www.cbd.int/ handbook.

SeCRETARIAT OF THE CBD (2006): Global biodiversity outlook 2. URL http://www. cbd.int/gbo2.

Secretariat of the CBD (2014): List of Parties. URL http://www.cbd.int/ information/parties.shtml. Retrieved 09/23/2014.

Statistische Ämter des Bundes und DeR LÄNder (2012): Flächennutzung. URL http:/ /www.statistik-portal.de/statistik-portal/de_jb09_jahrtabf1.asp.

Steinhardt, U.; Herzog, F.; Lausch, A.; Müller, E. \& Lehmann, S. (1999): Hemeroby index for landscape monitoring and evaluation. In: PYKH, Y. A.; НyATT, D. E. \& LENZ, R. J. (eds.) Environmental indices systems analysis approach, EOLSS, Advances in sustainable development. pp. 237-257.

Stevens, C. J.; Duprè, C.; Dorland, E.; Gaudnik, C.; Gowing, D. J. G.; Bleeker, A.; Diekmann, M.; Alard, D.; Bobbink, R.; Fowler, D.; Corcket, E.; Mountford, J. O.; VandviK, V.; Aarrestad, P. A.; Muller, S. \& Dise, N. B. (2010): Nitrogen deposition threatens species richness of grasslands across Europe. Environmental Pollution 158: 2940-2945. 
Stoate, C.; Boatman, N.; Borralho, R.; Carvalho, C. R.; Snoo, G. R. D. \& Eden, P. (2001): Ecological impacts of arable intensification in Europe. Journal of Environmental Management 63: 337-365.

Storkey, J.; Meyer, S.; Still, K. S. \& Leuschner, C. (2012): The impact of agricultural intensification and land-use change on the European arable flora. Proceedings of the Royal Society B - Biological Sciences 279: 1421-1429.

Suкopr, H. (1976): Dynamik und Konstanz in der Flora der Bundesrepublik Deutschland. Schriftenreihe für Vegetationskunde 10: 9-27.

Sukopp, H. \& StaRfinger, U. (1999): Disturbance in urban ecosystems. In: WalkeR, L. R. (ed.) Ecosystems of disturbed ground, Elsevier, Amsterdam. pp. 397-412.

The Secretariat of the CBD (2002): Global Strategy for Plant Conservation. Montreal.

Thomas, C. D.; Cameron, A.; Green, R. E.; Bakkenes, M.; Beaumont, L. J.; Collingham, Y. C.; Erasmus, B. F. N.; Siqueira, M. F. D.; Grainger, A.; Hannah, L.; Hughes, L.; Huntley, B.; JaArsveld, A. S. v.; Midgley, G. F.; Miles, L.; OrtegaHuerta, M. A.; Peterson, A. T.; Phillips, O. L. \& Williams, S. E. (2004): Extinction risk from climate change. Nature 427: 145-148.

Thuiller, W.; Lavorel, S.; Araújo, M. B.; Sykes, M. T. \& Prentice, I. C. (2005): Climate change threats to plant diversity in Europe. PNAS 102: 8245-8250.

Tilman, D.; Fargione, J.; Wolff, B.; D'Antonio, C.; Dobson, A.; Howarth, R.; Schindler, D.; Schlesinger, W. H.; Simberloff, D. \& Swackhamer, D. (2001): Forecasting agriculturally driven global environmental change. Science 292: 281284.

TscharntKe, T.; Klein, A. M.; Kruess, A.; Steffan-Dewenter, I. \& Thies, C. (2005): Landscape perspectives on agricultural intensification and biodiversity - ecosystem service management. Ecology Letters 8: 857-874.

Tscharntke, T.; Tylianakis, J. M.; Rand, T. A.; Didham, R. K.; Fahrig, L.; Batáry, P.; Bengtsson, J.; Clough, Y.; Crist, T. O.; Dormann, C. F.; Ewers, R. M.; Fründ, J.; Holt, R. D.; Holzschuh, A.; Klein, A. M.; Kleijn, D.; Kremen, C.; Landis, D. A.; Laurance, W.; Lindenmayer, D.; Scherber, C.; Sodhi, N.; Steffan-Dewenter, I.; Thies, C.; van der Putten, W. H \& Westphal, C. (2012): Landscape moderation of biodiversity patterns and processes - eight hypotheses. Biological reviews of the Cambridge Philosophical Society 87: 661-685.

UBA (Federal Environment Agency) (2014): Flächensparen - Böden und Landschaften erhalten. URL http://www.umweltbundesamt.de/themen/ 
boden-landwirtschaft/flaechensparen-boeden-landschaften-erhalten. Retrieved $09 / 16 / 2014$.

UNECE (United Nations Economic Commission for Europe) (1979): Convention on Long-range Transboundary Air Pollution. URL http://www. unece.org/fileadmin/DAM/env/lrtap/full\%20text/1979.CLRTAP.e.pdf. Retrieved $09 / 13 / 2014$.

UNEP (United Nations Environment Programme) (2014): Planning and management of lakes and reservoirs: An integrated approach to eutrophication: Chapter 5: Economic aspects of eutrophication. URL http://www.unep.or.jp/ietc/ publications/techpublications/techpub-11/5-3-1.asp. Retrieved 09/15/2014.

Vetaas, O. R. \& Ferrer-Castán, D. (2008): Patterns of woody plant species richness in the Iberian Peninsula: environmental range and spatial scale. Journal of Biogeography 35: 1863-1878.

WALZ, U. \& STEIN, C. (2014): Indicators of hemeroby for the monitoring of landscapes in Germany. Journal for Nature Conservation 22: 279-289.

WANG, Z.; FAnG, J.; TANG, Z. \& Lin, X. (2011): Patterns, determinants and models of woody plant diversity in China. Proceedings of the Royal Society B - Biological Sciences 278: 2122-2132.

WaniA, A.; KüHN, I. \& KLOTZ, S. (2006): Plant richness patterns in agricultural and urban landscapes in Central Germany - spatial gradients of species richness. Landscape and Urban Planning 75: 97-110.

White, P. J. T. \& KerR, J. T. (2007): Human impacts on environment-diversity relationships: evidence for biotic homogenization from butterfly species richness patterns. Global Ecology and Biogeography 16: 290-299.

WiENS, J. J. (2011): The causes of species richness patterns across space, time, and clades and the role of "ecological limits". The Quarterly Review of Biology 86: 75-96.

Winter, S.; Fischer, H. S. \& Fischer, A. (2010a): Relative quantitative reference approach for naturalness assessments of forests. Forest Ecology and Management 259: 1624-1632.

Winter, S.; JANTSCH, M. \& RAAB, B. (2010b): Ermittlung von Important Plant Areas in Deutschland. Natur und Landschaft 85: 105-110.

Wohlgemuth, T. (1998): Modelling floristic species richness on a regional scale: a case study in Switzerland. Biodiversity and Conservation 7: 159-177. 
WrbKa, T.; Erb, K.-H.; Schulz, N. B.; Peterseil, J.; Hahn, C. \& Haberl, H. (2004): Linking pattern and process in cultural landscapes. An empirical study based on spatially explicit indicators. Land Use Policy 21: 289-306.

Yamaura, Y.; Kawahara, T.; IIdA, S. \& OzaKI, K. (2008): Relative importance of the area and shape of patches to the diversity of multiple taxa. Conservation biology: the journal of the Society for Conservation Biology 22: 1513-1522.

ZAJAC, A. \& ZAJAC, M. (2001): Distribution atlas of vascular plants in Poland. Kraków. 714 pp.

Zebisch, M.; Grothmann, T.; Schröter, D.; Hasse, C.; Fritsch, U. \& Cramer, W. (2005): Climate change in Germany: Vulnerability and adaption strategies of climate-sensitive sectors: Summary. Research Report. 205 pp.

Zimbres, B.; Furtado, M. M.; Jácomo, A. T. A.; Silveira, L.; SollmanN, R.; TôRRes, N. M.; Machado, R. B. \& Marinho-Filho, J. (2013): The impact of habitat fragmentation on the ecology of xenarthrans (Mammalia) in the Brazilian Cerrado. Landscape Ecology 28: 259-269. 
Description of data 


\subsection{Study region}

The study region comprises the German federal states of Lower Saxony and Bremen. Lower Saxony encompasses an area of $47.624 \mathrm{~km}^{2}$ whereas Bremen as the smallest federal state of Germany has a size of only $404 \mathrm{~km}^{2}$. As landscape formation history and natural environmental configuration, colonisation history and current land use vary widely across the state, Lower Saxony harbours a high number of different habitats. The area may roughly be subdivided into three regions, the coastal, the lowland and the upland area (Fig. 2.1), that largely differ in their characteristics.

The coastal area is dominated by marine deposits from the Holocene (HeunisCH et al., 2007). It comprises the Wadden Sea, its islands and salt marshes, the estuaries of the three largest rivers of Lower Saxony (Ems, Weser and Elbe) as well as impoldered tidal wetlands. It shows an oceanic climate with mean annual precipitation rates of about 700-900 mm and mean annual temperatures of c. $9^{\circ} \mathrm{C}$. Main soil types occurring in this area are Fluvisols, Leptosols and Gleysols (NLFB, 1997 $)$. The costal area is predominantly covered by grasslands with a proportion of $60 \%$ whereas cropland areas contribute $20 \%$ and forests merely $1 \%$.

The lowland area was formed during the Pleistocene and harbours various types of landscapes ranging from intensively managed agricultural landscapes in the western part to more wooded landscapes in the eastern part. From West to East, the climate changes from oceanic (annual precipitation rate c. $900 \mathrm{~mm}$ ) to suboceanicsubcontinental (annual precipitation rate c. $550 \mathrm{~mm}$ ). Mean annual temperatures in this area fluctuate around $8^{\circ} \mathrm{C}$. The climatic gradient is reflected in the soil characteristics: In the West the dominating soil types are those affected by seepage water, water logging and mass transfer (Histosols, Podzols); in the East primarily Cambisols and Leptosols are found. The area is dominated by croplands that cover about $40 \%$ of this region; forests and grasslands are present at proportions of about $20 \%$ each.

The upland area is a very heterogeneous region that is dominated by deposits from the Meso- and Palaeozoic (Heunisch et al., 2007). Due to its diverse topography, climatic conditions vary strongly. The uplands are dominated by aeolian silt as well as clay, sand and lime stone deposits (NLFB, 1997). Luvisols and Cambisols are the most common soil types in this region. Mean annual temperatures range between $8-9^{\circ} \mathrm{C}$. Mean annual precipitation rates vary between $650-750 \mathrm{~mm}$ in the lower parts of the area and $800-900 \mathrm{~mm}$ in the elevated parts. The Harz mountains at the South-western border of Lower Saxony feature very specific climatic conditions: The most elevated parts (up to $971 \mathrm{~m}$ a.s.l.) experience a montane climate with an annual mean precipitation rate of up to $1,500 \mathrm{~mm}$ and annual mean temperatures of only $7^{\circ} \mathrm{C}$ (NLFB, 1997). The Harz region is dominated by base poor silicate bedrock as well as argillaceous shale and greywacke. Main soil types occurring in this area are Cambisols and Podzols (NLFB, 1997). Out of the three regions, the upland area features the high-

\footnotetext{
${ }^{1}$ Translation of German soil types follows IUSS WorkING Group WRB (2006).
} 
est proportion of forests (c. $30 \%$ ); grasslands only prevail in about $9 \%$ of the area whereas croplands dominate c. $45 \%$ of the region.

The study region was subdivided into 1762 grid cells (Messtischblatt-Quadranten) with a size of about $5.5 \mathrm{~km} \times 5.5 \mathrm{~km}$ (1/4 of a topographical map, scale $1: 25,000$; Fig. 2.1). Out of these 1386 grid cells are fully covered by land, 353 grid cells are located on the edge and 23 grid cells are fully covered by sea. For a more detailed study (see chapter 4, Schmiedel et al., 2013), the lowland area of the state was overlaid with a more detailed grid of 15.729 cells with a size of about $1 \mathrm{~km} \times 2 \mathrm{~km}$ (Minutenfelder, $1 / 60$ of a topographical map, scale $1: 25,000$ ).

\subsection{Data and data preparation}

Data available for the study region included information on the distribution of vascular plant species, data on environmental variables as well as land-use types (Table 2.1). The following paragraphs give a detailed overview of these data.

Species data were analysed using MS Access (Microsoft Corporation, 2010) and ArcGIS-ArcInfo 9.2 (ESRI INC., 2007). ATKIS data, areal information as well as environmental variables - unless stated otherwise - were analysed using ArcGIS-ArcInfo 9.2, Hawth's Tools (Beyer, 2004) and Fragstats 3.3 (McGarigal \& Cushman, 2002).

\subsubsection{Species data}

Occurrence data of all vascular plant species of Lower Saxony and Bremen were made available as an MS Access database by the vascular plant survey of Lower Saxony (Niedersächsisches Pflanzenartenerfassungsprogramm, NLWKN; Table 2.1). The data base (last update 15 December 2008) comprises 2,395,045 entries and covers the time span from 1993-2003 (first and second period of the state-wide mapping; third period is still underway). Out of all taxa listed in the data base only the $1879^{2}$ non-erratic ones were considered ${ }^{3}$. Occurrence data were available on the grid basis mentioned in the previous chapter.

Prior to the analyses, species were aggregated on the highest taxonomical level found in the data base, i. e. subspecies were assigned to the species level and species to the respective aggregate found in the data base ${ }^{4}$. This aggregation affected 213 taxa (see Table A.3 in Appendix A for an overview). Thus, in total, 1666 taxa remained in the analysis out of which 72 were occurring in grid cells fully covered by sea or located on the edge of the study area only ${ }^{5}$ (cp. section 2.1) and thus were excluded

\footnotetext{
${ }^{2}$ Due to errors in the database that were detected just after the first article (Schmiedel et al., 2011) was published, the total number of species considered in this article was 1819 taxa only.

${ }^{3}$ In total, 2708 taxa are listed in the floristic list of the data base out of which 829 are extinct, erratic or do not occur.

${ }^{4}$ The exception being the first article (ScHMIEDEL et al., 2011) which did not apply any aggregation.

${ }^{5}$ Hereafter referred to as 'edge only' species.
} 
for the analyses conducted in Schmiedel \& Culmsee (submitted) and Schmiedel et al. (submitted).

For the analyses conducted in context of the present thesis, the species listed in the data base were aggregated into sub-groups using the residence and threat status and habitat preferences of the species, respectively (Table 2.2).

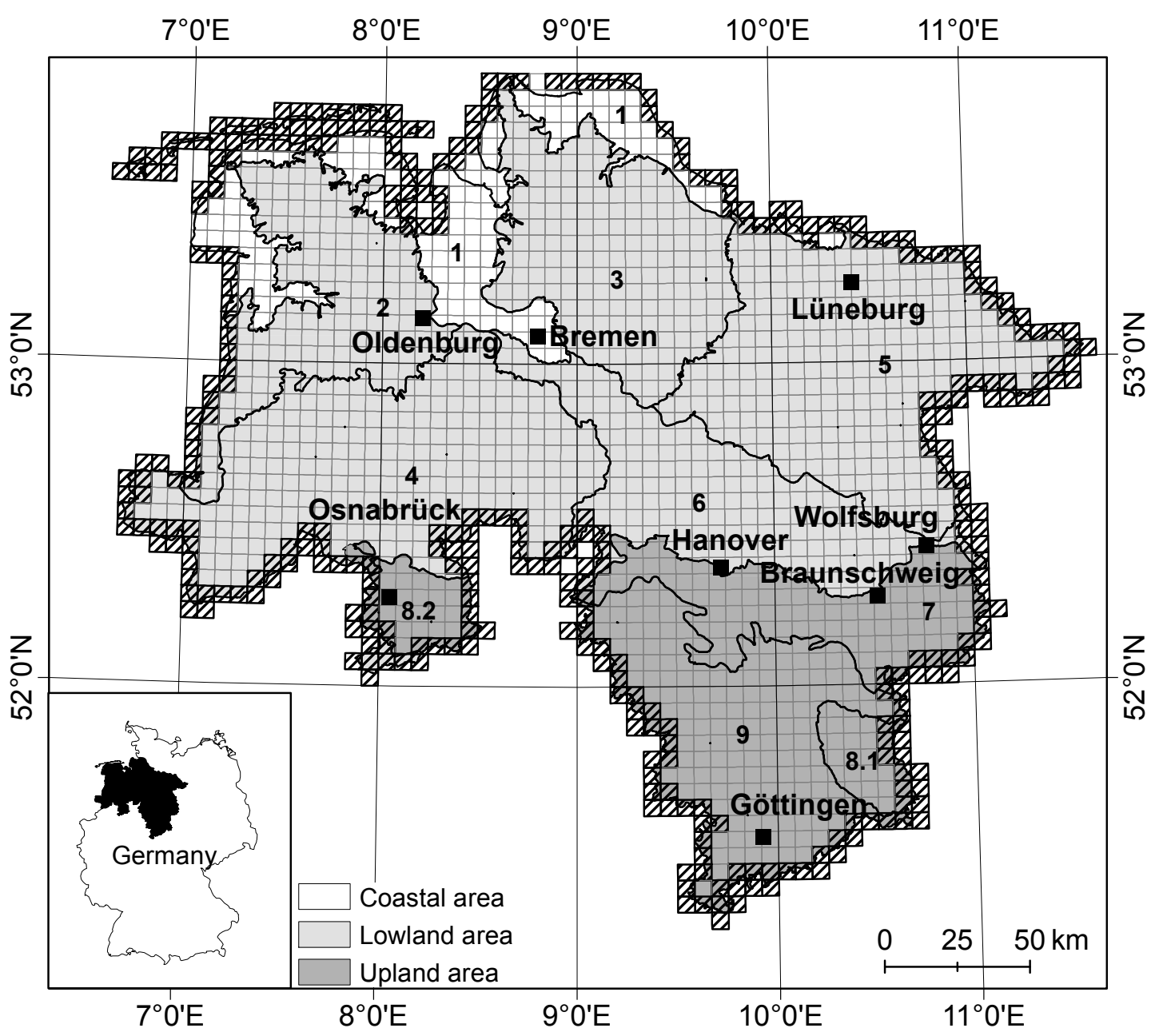

Figure 2.1: The study area encompassing the federal states of Lower Saxony and Bremen, with its three natural landscape regions (coastal, lowland and upland area). The region can be further subdivided into 10 bio-geographical regions $(1=$ Wadden Sea and marshes, $2=$ East Frisian and Oldenburg moraine lowlands, $3=$ Stade moraine lowlands, $4=$ Ems, Hunte and Dümmer moraine lowlands, $5=$ Luneburg Heath and Wendland, $6=$ Weser and Aller plains, $7=$ Old moraine loess landscapes, $8.1=$ Uplands of Osnabrück, $8.2=$ Uplands of the Weser and Leine valleys, $9=$ Harz mountains.) Plant occurrence data from the plant survey of Lower Saxony were available for 1762 quadrants of the topographical map (scale 1:25,000, i. e. about $5.5 \mathrm{~km} \times 5.5 \mathrm{~km}$ in size). The area of hatched quadrants has only been partly inventoried, because they are either only partially covered by land area or are situated in neighbouring federal states. 
The residence status of species was determined by using the information provided by the data base itself and information from KLOTz et al. (2002): Native species were distinguished from archaeophytes (species that became naturalised prior to the $16^{\text {th }}$ century) and neophytes (species that became naturalised in the $16^{\text {th }}$ century or later) (see Table 2.2). Information on the threat status of species was derived from the Red List of Lower Saxony and Bremen (GARve, 2004): Threatened species (Red List status $1,2,3$, G and R) were distinguished from non-threatened species (Red List status V and ${ }^{*}$ ) (see Table 2.2).

Information on habitat preference, i. e. the linkage to urban and forest habitats were taken from KLOTZ \& KüHN (2002), BFN (2013) and SCHMIDT et al. (2011), respectively (Table 2.2). Urban species were defined as those species with an urbanity indicator value of 4 (urbanophilic) and 5 (moderately urbanophilic). Forest species are those species categorised as to occur in closed forests only (category 1.1, ScHMIDT et al., 2011) as well as forest edges and clearings (category 1.2, Sснмidt et al., 2011). Information on Ellenberg Indicator Values (EIV) for nutrients (N) were taken from BFN (2013). Those species having a $\mathrm{N}$ value of 1 to 3 were defined as species of nutrientpoor habitats, those with a $\mathrm{N}$ value of 7 to 9 as species of nutrient-rich habitats (Table 2.2).

Table A.2 in Appendix A lists all species, along with their residence and threat status as well as their habitat preferences and furthermore denotes the 'edge only' species.

Pivot tables in MS Access were used to compute species richness per grid cell. One query was conducted for each species group (Table 2.2).

\subsubsection{ATKIS data}

Information on the extent and spatial configuration of different land use types were derived from the ATKIS Digital Landscape Model (DLM, LGN, 2008) of Lower Saxony and Bremen. Overall, the DLM differentiates c. 90 different 'ATKIS object types'. These were combined into 8 different land cover types (LCTs), i. e. industrial, urban and traffic areas, croplands, forests, grasslands, water bodies and "other LCTs" as listed in Table 2.3. Linear elements represented as polylines in the DLM (roads, rivers) were buffered by using either standard values (roads, FGSV, 1996, see. Table A.5 in Appendix A) or information provided by the dataset itself (rivers) in order to convert them into polygons (Hawth's Analysis Tools, BEYER, 2004). Subsequently, they were combined with the other polygon features to a non-overlapping feature layer using ArcGIS-ArcInfo 9.2 (ESRI INC., 2007).

Information on average altitude, inclination and exposure per grid cell were derived from the ATKIS Digital Elevation Model (DEM, LGN, 2008). The DEM data were further used to calculate the Mean Heat Load Index (McCune \& KEON, 2002, eq. 3) per grid cell by using the ArcScript 'Heat load index' (PARKs, 2004) in ArcView GIS 3.3 (ESRI INC., 2002). 


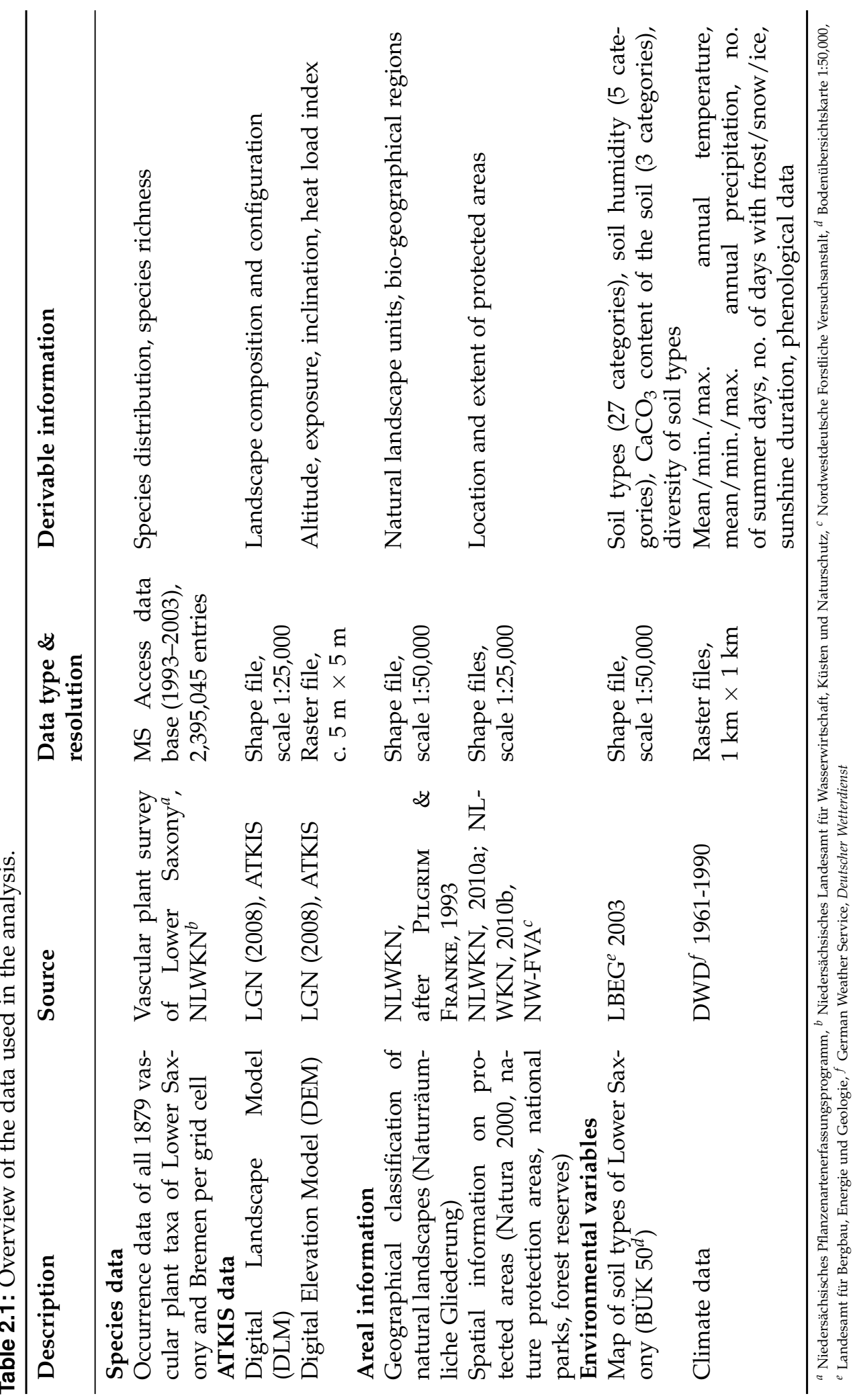




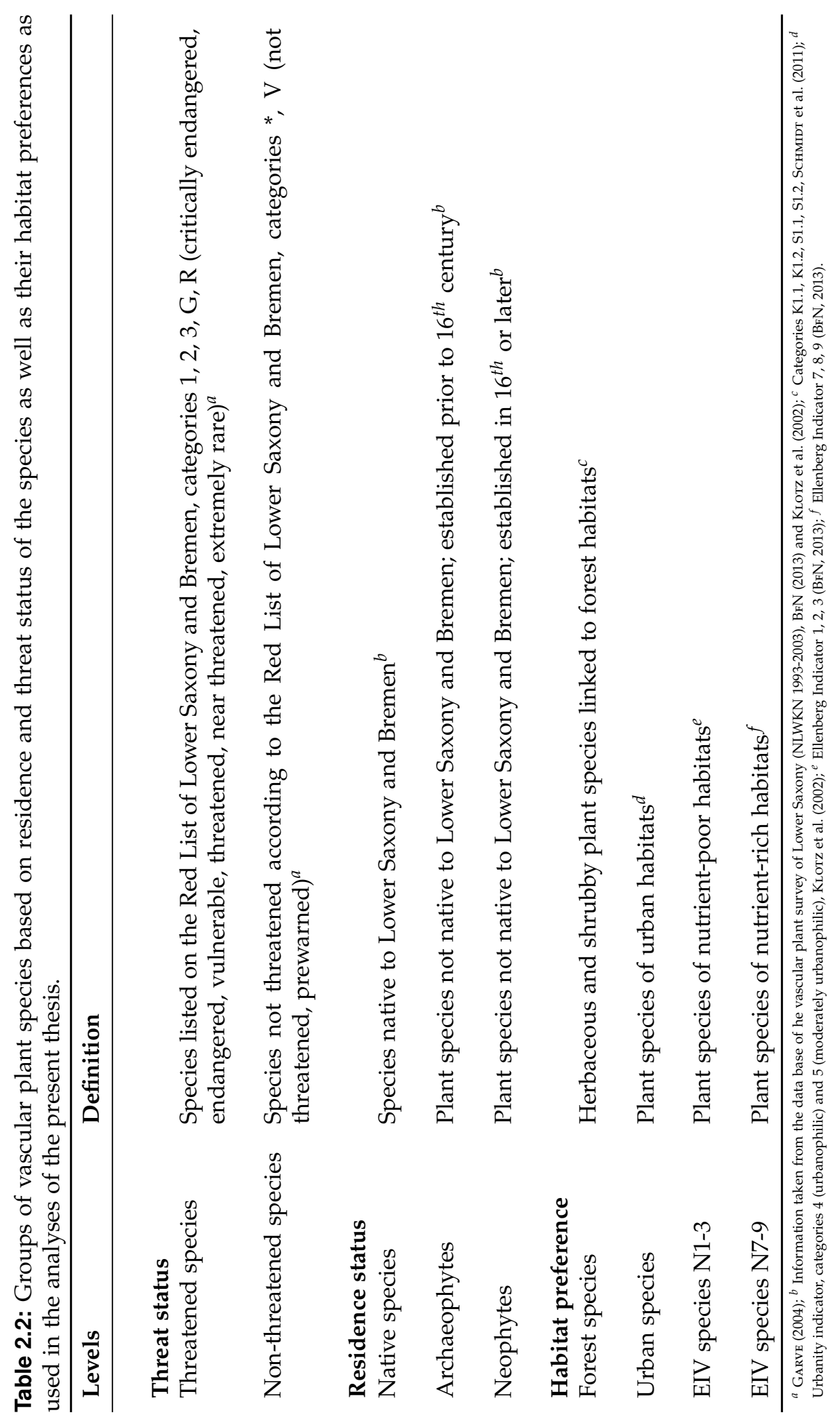




\subsubsection{Areal information}

Information on bio-geographic regions and the location and extent of protected areas were obtained from shape files provided by the NLWKN and the NW-FVA (Table 2.1).

The geographical classification of natural landscapes of Lower Saxony (PILGRIM \& FRANKE, 1993) defines the borders of the natural landscape units of the state. Roughly, the state area can be divided into three regions (coastal, lowland and upland area, cp. section 2.1) which themselves can be further divided into 10 bio-geographical regions and 82 natural landscape units (see Table A.1 in Appendix A). Because the grid cells in some cases were placed on the border between two or several regions/units the following rule was applied: Each grid cell was defined to belong to that region/unit that holds the highest proportion of area of the respective grid cell.

Information on protected areas included the location and extent of Natura 2000 sites, nature protected areas (Naturschutzgebiete, NSG), national parks as well as forest reserves (Naturwaldreservate) (Table 2.1).

\subsubsection{Environmental variables}

Soils Information on soil types, soil humidity, $\mathrm{CaCO}_{3}$ content as well as the diversity of soil types were obtained from the map of soil types of Lower Saxony (BÜK 50, scale 1:50,000, vector data; Table 2.1). For all calculations the vector data were transformed into raster data of a pixel size of $50 \mathrm{~m} \times 50 \mathrm{~m}$.

BÜK 50 lists 126 soil types that were grouped into 27 soil type categories (see Table A.4 in Appendix A). Based on these categories, the diversity of soil types was calculated using Fragstats 3.3 (MCGARIGAL \& Cushman, 2002).

Soil humidity in BÜK 50 is arranged in 11 levels ranging from arid to wet/open water conditions. An additional $12^{\text {th }}$ level includes all soils influenced by backwater. The 12 levels were grouped into 5 categories: "dry" (BÜK 50, soil moisture levels 0-3), "mesic" (soil moisture levels 4-6), "moist" (soil moisture levels 7-9), "wet" (soil moisture levels 10-11) and "influenced by backwater" (soil moisture level 12). Finally, the surface ratio of each soil moisture category was calculated per grid cell.

The $\mathrm{CaCO}_{3}$ content of the soil in $\mathrm{BÜK} 50$ is arranged in six levels ranging from soils with no $\mathrm{CaCO}_{3}$ content (BÜK 50, level $\mathrm{c} 0$ ) to soils with a high $\mathrm{CaCO}_{3}$ content (level c5). These levels were grouped into three categories: "free from $\mathrm{CaCO}_{3}$ " (BÜK 50 $\mathrm{CaCO}_{3}$ content level $\left.\mathrm{c} 0, \mathrm{c} 1\right)$, "medium $\mathrm{CaCO}_{3}$ content" (c2, c3) and "high $\mathrm{CaCO}_{3}$ content" ( $c 4, c 5)$. Then, the surface ratio of each $\mathrm{CaCO}_{3}$ category was calculated per grid cell.

Climate Information on regional climate were obtained from climate data of the German Weather service (Deutscher Wetterdienst, DWD; Table 2.1). Several different climatic parameters were derivable, i. e. mean, minimum and maximum annual temperature and precipitation, the number of summer days and days with frost, snow 
Table 2.3: Land cover types (LCTs) derived from ATKIS-DLM. For more detailed information on the included ATKIS object types see Table A.6 in Appendix A.

\begin{tabular}{|c|c|}
\hline LCT & Description \\
\hline Industrial areas & $\begin{array}{l}\text { Areas used for (non agricultural) production (e. g. supply and disposal } \\
\text { facilities, exhibition centers, mining areas etc.) }\end{array}$ \\
\hline Urban areas & $\begin{array}{l}\text { Areas used for housing and recreation (e.g. residential areas, parks, } \\
\text { campgrounds etc.) }\end{array}$ \\
\hline Traffic areas & Areas used for transport (roads, railway lines, airports, etc.) \\
\hline Croplands & Areas used for agricultural production \\
\hline Forests & Forested areas \\
\hline Grasslands & Grassland areas \\
\hline Water bodies & Standing and flowing waters (e. g. lakes, streams, channels, springs) \\
\hline Others & $\begin{array}{l}\text { Areas not belonging to any of the above mentioned LCTs (e. g. tree rows, } \\
\text { bogs, islands) }\end{array}$ \\
\hline
\end{tabular}

and ice as well as the sunshine duration. Furthermore, phenological data, e.g. the time of snowdrop (Galanthus nivalis) flowering, apple blossom etc. were available. All parameters were calculated as averages per grid cell.

\subsubsection{Landscape metrics}

Landscape metrics were used to quantify the composition and configuration of the landscape in the study area. These metrics are based on the patch-corridor-matrix concept by FORMAN (1995) which states that landscapes are composed of structural elements: the patches, the corridors and the matrix. The arrangement of these elements in the landscape creates a particular landscape pattern or patch mosaic (LANG \& BlaschKe, 2007). The term 'patch' in this context describes more or less homogeneous, non-linear landscape elements that differ from the surrounding landscape (LeItão et al., 2006). The matrix is defined by its proportion of the landscape ( $\geq 50 \%)$, its connectivity as well as its control on landscape dynamics.

Landscape metrics that evolved in the 1980s and 90s (LANG \& BLASCHKE, 2007) facilitate the description of patterns of a landscape with regard to its composition and configuration on the patch, class as well as landscape level. These indices describe and quantify the spatial structure of patches, classes and the landscape, respectively (LeItÃo et al., 2006). They provide the opportunity to measure geometric properties of landscape elements and their relative position and distribution in the landscape (Leitão et al., 2006). On the patch level, the geometric properties of individual patches can be assessed, whereas on the class level patches of the same type (class; in this study named land cover types, LCTs) are aggregated. On the landscape level the configuration of different types of classes is considered (LANG \& BLASCHKE, 2007).

All landscape metrics were calculated using Fragstats 3.3 (McGarigal \& CusHMAN, 2002). Because the software is limited to the use of raster data, the DLM vector 
data were converted to raster format with a resolution of $5 \mathrm{~m} \times 5 \mathrm{~m}$ using ArcGISArcInfo 9.2 (ESRI INC., 2007). A higher resolution could not be chosen because of hardware limitations. Because landscape metrics had to be assessed separately for each of the 1762 grid cells (see section 2.1) the raster data set was cut into 1762 separate rasters using Hawth's Tools (BEYER, 2004) in ArcGIS. In the following these 1762 tiles were considered as separate 'landscapes'. Landscape metrics were calculated on class and landscape level, respectively. The indices that were calculated describe the landscape composition (e.g. patch sizes, patch numbers) and configuration, i.e. the interactions and correlations between the landscape elements (e. g. expressed in terms of patch shape, complexity, fragmentation). The following paragraphs name and describe the landscape metrics used to quantify the composition and configuration of the landscape. Naming of the indices follows the nomenclature used in Fragstats 3.3 (McGarigal et al., 2002).

\section{Landscape metrics quantifying landscape composition}

Class level The landscape composition within the 1762 grid cells on the class level was assessed using the indices Total Class Area (CA) and Number of Patches (NP; Table 2.4). CA for each landscape reports the percentage of area of the particular classes (McGarigal \& Cushman, 2002). NP counts the number of patches of each class under observation. The NP index together with CA represents a simple measure to quantify fragmentation of the classes in the landscape: If a class is characterised by high values of CA as well as NP the area belonging to the class is highly fragmented.

Landscape level The landscape composition within the 1762 grid cells on the landscape level was assessed using the indices Patch Richness (PR), Shannon's Diversity Index (SHDI) and Shannon's Evenness Index (SHEI; Table 2.4). PR addresses the number of classes in the landscape and thus is an important basic measure for landscape diversity. If besides the pure number of classes in the landscape also their proportions shall be considered, the Shannon's Diversity Index is the method of choice: If the landscape is composed of one patch only, the value of SHDI equals zero. The more patch types are present and the more homogeneously distributed they are, the larger the value of SHDI becomes. SHEI, in turn, is a measure of the evenness of the distribution of patch types/classes (McGarigal \& Cushman, 2002). The index can take values between zero and one: If a landscape consists of only one patch type, values of SHEI will be zero. If several LCTs are present, the value of SHEI increases the more evenly distributed they are.

Table 2.4 lists all landscape metrics used to assess landscape composition, their range of values as well as their units. 
Table 2.4: Landscape metrics used to assess landscape composition with respect to their range of values, units and basis of computation (after MCGARIGAL \& CUSHMAN, 2002). LCT = land cover type; $\uparrow=$ increase in value; $\mathrm{n} / \mathrm{a}=$ not available.

\begin{tabular}{llrr}
\hline $\begin{array}{l}\text { Landscape } \\
\text { parameter }\end{array}$ & $\begin{array}{l}\text { Landscape } \\
\text { metric }\end{array}$ & Range of values & Unit $\begin{array}{l}\text { Basis of } \\
\text { computation }\end{array}$ \\
\hline
\end{tabular}

\section{Class level}

\begin{tabular}{|c|c|c|c|c|}
\hline Total class area & Total Class Area, CA & $\mathrm{CA}>0$ & ha & All LCTs \\
\hline \multirow[t]{2}{*}{$\begin{array}{l}\text { Patch numbers/ } \\
\text { Landscape } \\
\text { diversity }\end{array}$} & $\begin{array}{l}\text { Number of Patches, } \\
\text { NP }\end{array}$ & $\mathrm{NP} \geq 1$ & $\mathrm{n} / \mathrm{a}$ & $\begin{array}{l}\text { grassland } \\
\text { cropland, } \\
\text { forest }\end{array}$ \\
\hline & & $\begin{array}{l}\mathrm{NP}=1 \text {, if the landscape } \\
\text { consists of one single patch }\end{array}$ & & \\
\hline \multicolumn{5}{|l|}{ Landscape level } \\
\hline $\begin{array}{lr}\text { Number } & \text { of } \\
\text { patch } & \text { types } \\
\text { (classes) } & \end{array}$ & Patch Richness, PR & $\mathrm{PR}>0$ & $\mathrm{n} / \mathrm{a}$ & All LCTs \\
\hline \multirow[t]{2}{*}{$\begin{array}{l}\text { Landscape } \\
\text { diversity }\end{array}$} & $\begin{array}{l}\text { Shannon's Diversity } \\
\text { Index, SHDI }\end{array}$ & SHDI $\geq 0$ & $\mathrm{n} / \mathrm{a}$ & All LCTs \\
\hline & & $\begin{array}{l}\text { SHDI }=0 \text {, if the landscape } \\
\text { consists of one patch/class } \\
\text { only } \\
\text { SHDI } \uparrow \text {, if the number } \\
\text { of patch types/classes in- } \\
\text { creases and/or the propor- } \\
\text { tions of different classes is } \\
\text { becoming more uniformly } \\
\text { distributed }\end{array}$ & & \\
\hline \multirow[t]{2}{*}{$\begin{array}{l}\text { Uniform distri- } \\
\text { bution of LCTs }\end{array}$} & $\begin{array}{l}\text { Shannon's Evenness } \\
\text { Index, SHEI }\end{array}$ & $0 \leq \mathrm{SHEI} \leq 1$ & $\mathrm{n} / \mathrm{a}$ & All LCTs \\
\hline & & $\begin{array}{l}\text { SHEI }=0 \text {, if the landscape } \\
\text { consists of one patch/class } \\
\text { only } \\
\text { SHEI } \uparrow \text {, if the proportion of } \\
\text { the patches of all classes } \\
\text { are becoming more equal } \\
\text { SHEI }=1 \text {, if the patches } \\
\text { of all classes are present at } \\
\text { the same proportions }\end{array}$ & & \\
\hline
\end{tabular}




\section{Landscape metrics quantifying landscape configuration}

Class level Landscape configuration on the class level was assessed only for selected LCTs, i. e. forest, grassland and cropland areas. For these LCTs several configuration indices, namely the Area-weighted Mean Patch Area Distribution (AREA_AM), the Areaweighted Mean Shape Index (SHAPE_AM), the Total Edge Index (TE), the Mean Proximity Index (PROX_MN), the Total Core Area Index (TCA) as well as the Area-weighted Mean Core Area Distribution (CORE_AM) were calculated (Table 2.5). In the present study metrics based on area-weighted mean (AM) patch characteristics were favoured over those describing the average $(\mathrm{MN})$ patch characteristics because the former provide a more landscape-centric instead of patch-centric perspective and thus better reflect the average conditions of a randomly chosen pixel in the landscape (MCGARIGAL, 2014).

AREA_AM calculates the area-weighted mean size of all patches of one class. Thus, the index allows to discriminate between LCTs present at a large vs. small scale (McGarigal \& Cushman, 2002).

SHAPE_AM quantifies the complexity of patches. In raster data the simplest shape is a square; the more complex a patch becomes, i.e. the more it deviates from the shape of a square, the stronger the value of the Shape Index increases.

PROX_MN quantifies the isolation or rather connectivity of the patches of one class. For the calculation of the index a 'search radius' has to be defined; patches within this distance are regarded as connected. The search radius in the study was set to $100 \mathrm{~m}$. If a patch has no neighbours of the same patch type within this distance the Proximity Index is 0; the index increases as the neighbourhood of the patch is increasingly occupied by patches of the same type; the closer these patches become and the less scattered they occur, the larger the value of the index becomes (McGARIGAL, 2014).

TE sums up the length of the edges of all patches belonging to one LCT (LANG \& BLASCHKE, 2007). A high value of TE can - depending on the quality of the LCT under consideration - either be an indicator of a high structural diversity or identify a high degree of landscape fragmentation (LANG \& BLASCHKE, 2007).

TCA sums up the core areas of all patches belonging to one class. The core area is defined as the area within a specified core area distance, i.e. that area that is not influence by edge effects (McGarigal \& CUSHMAN, 2002; LANG \& BLASCHKE, 2007). The core area of patches, different from patch size, is influenced by the shape of the patches. Thus, a patch can be potentially large enough to support particular species but because of its shape can be influenced by edge effects to such a large extent that the habitat becomes unsuitable for species (McGarigal \& Cushman, 2002). For the calculation of TCA the 'edge depth' has to be defined. In the present study TCA was calculated for forests only; edge depth was defined as being $100 \mathrm{~m}$ (cp. MURCIA, 1995; Harper et al., 2005; Hennenberg et al., 2005; Wei \& Hoganson, 2005; Hennenberg et al., 2008a,b). CORE_AM resembles TCA but measures the average core area of all patches belonging to one class. 
Table 2.5: Landscape metrics used to assess landscape configuration with respect to their range of values, units and basis of computation (after MCGARIGAL \& CUSHMAN, 2002). FGs = fragmentation geometries. $\mathrm{LCT}=$ land cover type; $\uparrow=$ increase in value; $\mathrm{n} / \mathrm{a}=$ not available.

\begin{tabular}{llcl}
\hline $\begin{array}{l}\text { Landscape } \\
\text { parameter }\end{array}$ & Landscape metric & Range of values & $\begin{array}{l}\text { Unit } \\
\begin{array}{l}\text { Basis of com- } \\
\text { putation }\end{array}\end{array}$ \\
\hline
\end{tabular}

\section{Class level}

Patch complexity Shape Index Distribution, SHAPE_AM

Mean patch size

Patch Area Distribution, AREA_AM

Total Core Area Total Core Area TCA

Mean core area

Core Area Distribution, CORE_AM

Total edge length Total Edge, TE

Patch isolation
Proximity PROX_AM
SHAPE_AM $\geq 1$ SHAPE_AM $=1$, if the patches are maximally compact (square) SHAPE_AM $\uparrow$, if the shape of patches becomes more irregular

$$
\text { AREA_AM > } 0
$$

TCA $\geq 0$

$\mathrm{TCA}=0$, if each location of all patches belonging tot he class lays within the specified edge depth

CORE_AM $>0$

$\mathrm{TE} \geq 0$

\section{PROX $\geq 0$}

PROX $=0$, if a patch has no neighbours of the same patch type within the specified search radius n/a grassland, for-
est, cropland

ha grassland, forest, cropland ha forest

ha forest

m grassland, forest, cropland

$\mathrm{n} / \mathrm{a}$ grassland, forest, cropland

\section{Landscape level}

Complexity of Shape Index Distribupatches tion, SHAPE_AM
SHAPE_AM $\geq 1$ SHAPE_AM $=1$, if the patches are maximally compact (quadrat) SHAPE_AM $\uparrow$, if the shape of patches becomes more irregular

Mean patch size Patch Area Distribution, AREA_AM

Total edge length Total Edge, TE Landscape frag- Effective Mesh Size, mentation
AREA_MN $>0$

$\mathrm{TE} \geq 0$

$2.5 \times 10^{-3}$

MESH $\leq$ size of the grid cell $\mathrm{n} / \mathrm{a} \quad$ all LCTs

ha all LCTs

$\mathrm{m} \quad$ all LCTs

ha FGs \& remaining landscape

\footnotetext{
${ }^{a}$ Area of one single pixel of the raster (pixel $5 \mathrm{~m} \times 5 \mathrm{~m}=25 \mathrm{~m}^{2}=2.5 \times 10^{-3} \mathrm{ha}$ ).
} 
Landscape level Landscape configuration on the landscape level was assessed using the Area-weighted Mean Shape Index (SHAPE_AM), the Area-weighted Mean Patch Area Distribution (AREA_AM) as well as the Effective Mesh Size Index (MESH; later following JAEgER et al. (2006) also named $m_{\text {eff }}$ ) (Table 2.5).

As explained for the class level, SHAPE_AM quantifies the complexity of patches. On the landscape level this complexity is not averaged over the patches of one class but over all patches being present in the landscape. The same applies to AREA_AM that on the landscape level calculates the area-weighted mean size of all patches in the landscape and, in doing so, provides a measure of the overall fragmentation of LCTs.

MESH measures the fragmentation of a landscape and is frequently used in many studies quantifying landscape fragmentation (e. g. JAEGER et al., 2001; WALZ, 2005; EsSWEIN \& SCHWARZ-Von RAUMER, 2006; Moser et al., 2007; JAEger et al., 2008; Girvetz et al., 2008; LI et al., 2010; EEA, 2011). The index denotes the size of the patches when the landscape is divided into $S$ areas (each of the same size) with the same degree of landscape division as obtained for the observed cumulative area distribution (McGarigal, 2014).

Table 2.5 lists all landscape metrics used to assess landscape configuration, their range of values as well as their units. 


\section{References}

Beyer, H. L. (2004): Hawth's Analysis Tools for ArcGIS. URL http://www. spatialecology.com/index.php.

BfN (Bundesamt für Naturschutz) (2013): FloraWeb: Daten und Informationen zu Wildpflanzen und zur Vegetation Deutschlands. URL http://www.floraweb.de. Retrieved 07/01/2013.

DWD (1961-1990): Gridded data sets of long-term area averages in Germany: Resolution $1 \mathrm{~km}^{2}$ : Updated 05 May 2009.

EEA (European Environment Agency) (2011): Landscape fragmentation in Europe: Joint EEA-FOEN report, EEA Report, vol. 2. URL http://www.eea.europa. $\mathrm{eu} /$ publications/landscape-fragmentation-in-europe. Retrieved 05/15/2013.

ESRI INC. (2002): ArcGIS-ArcView 3.3.

ESRI INC. (2007): ArcGis-ArcInfo 9.2.

Esswein, H. \& Schwarz-von Raumer, H.-G. (2006): Darstellung und Analyse der Landschaftszerschneidung in Bayern. Endbericht im Auftrag des Bayerischen Landesamtes für Umweltschutz. URL http://www.lfu.bayern.de/natur/ landschaftszerschneidung/unzerschnittene_raeume/doc/bericht_lz_bz_06.pdf.

FGSV (ForschUNGSGESELlSCHAFT FÜR STRASSEN- UND VERKEHRSWESEN) (1996): Richtlinien für die Anlage von Straßen, Teil: Querschnitte (RAS-Q 96).

Forman, R. T. T. (1995): Land mosaics: The ecology of landscapes and regions. Cambridge Univ. Press, Cambridge. 632 pp.

GARve, E. (2004): Rote Liste und Florenliste der Farn- und Blütenpflanzen in Niedersachsen und Bremen. Informationsdienst Naturschutz Niedersachsen 24: 1-76.

Girvetz, E. H.; Thorne, J. H.; Berry, A. M. \& JAeger, J. A. G. (2008): Integration of landscape fragmentation analysis into regional planning: A statewide multi-scale case study from California, USA. Landscape and Urban Planning 86: 205-218.

Harper, K. A.; Macdonald, S. E.; Burton, P. J.; Chen, J. Q.; Brosofske, K. D.; SaunDers, S. C.; Euskirchen, E. S.; Roberts, D.; Jaiteh, M. S. \& Esseen, P. A. (2005): Edge influence on forest structure and composition in fragmented landscapes. Conservation Biology 19: 768-782.

HennenberG, K. J.; Goetze, D.; Kouame, L.; Orthmann, B. \& Porembski, S. (2005): Border and ecotone detection by vegetation composition along forest-savanna transects in Ivory Coast. Journal of Vegetation Science 16: 301-310. 
HennenberG, K. J.; Goetze, D.; Szarzynski, J.; Orthmann, B.; Reineking, B.; SteINKe, I. \& PoRembski, S. (2008a): Detection of seasonal variability in microclimatic borders and ecotones between forest and savanna. Basic and Applied Ecology 9: 275-285.

Hennenberg, K. J.; Orthmann, B.; Steinke, I. \& Porembski, S. (2008b): Core area analysis at semi-deciduous forest islands in the Comoé National Park (Ivory Coast). Biodiversity and Conservation 17: 2787-2797.

Heunisch, C.; Caspers, G.; Elbracht, J.; Langer, A.; Röhling, H.-G.; Schwarz, C. \& STREIF, H. (2007): Erdgeschichte von Niedersachsen: Geologie und Landschaftsentwicklung. GeoBerichte 6: 3-85.

IUSS WorkING GRoup WRB (2006): World reference base for soil resources 2006: A framework for international classification, correlation and communication, vol. 103. FAO, Rome. 128 pp.

Jaeger, J.; Esswein, H. \& Schwarz-von Raumer, H.-G. (2006): Measuring landscape fragmentation with the Effective Mesh Size $m_{e f f}$. Brochure. URL http://www.fragmentation.de/English/Publications/publications-Dateien/ documents/faltblatt_engl\%20.pdf. Retrieved 03/16/2014.

Jaeger, J. A. G.; Bertiller, R.; Schwick, C.; Müller, K.; Steinmeier, C.; Ewald, K. C. \& Ghazoul, J. (2008): Implementing landscape fragmentation as an indicator in the Swiss Monitoring System of Sustainable Development (MONET). Journal of Environmental Management 88: 737-751.

Jaeger, J. A. G.; Esswein, H.; Schwarz-von Raumer, H.-G. \& Müller, M. (2001): Landschaftszerschneidung in Baden-Württemberg. Naturschutz und Landschaftsplanung 33: 1-13.

KLOtZ, S. \& KüHN, I. (2002): Soziologische Bindung der Arten. Schriftenreihe für Vegetationskunde 38: 273-281.

KLOTZ, S.; KÜHN, I. \& DURKA, W. (2002): BIOLFLOR - Eine Datenbank zu biologischökologischen Merkmalen der Gefäßpflanzen in Deutschland. Schriftenreihe für Vegetationskunde 38: 1-334. URL http://www.biolflor.de.

LANG, S. \& BlaschKe, T. (2007): Landschaftsanalyse mit GIS. Ulmer, Stuttgart. 404 pp.

LBEG (LAndesamt für Bergbau, Energie und Geologie) (2003): Digitale Bodenkundliche Übersichtskarte 1: 50000 (BÜK50).

Leitão, A. B.; Miller, J. N.; Ahern, J. \& McGarigal, K. (2006): Measuring landscapes: A planner's handbook. Island Press, Washington. 245 pp. 
LGN (LANDESVERMESSUNG UND GeObasisinformation Niedersachsen) (2008): Amtliches Topographisch-Kartographisches InformationsSystem (ATKIS): Updated 17 December 2008.

Li, T.; Shilling, F.; Thorne, J. H.; Li, F.; Schott, H.; Boynton, R. \& Berry, A. M. (2010): Fragmentation of China's landscape by roads and urban areas. Landscape Ecology 25: 839-853.

McCune, B. \& Keon, D. (2002): Equations for potential annual direct incident radiation and heat load. Journal of Vegetation Science 13: 603-606.

McGarigal, K. (2014): Fragstats help. URL http://www.umass.edu/landeco/ research/fragstats/documents/fragstats.help.4.2.pdf. Retrieved 03/02/2014.

McGarigal, K. \& Cushman, S. A. (2002): Comparative evaluation of experimental approaches to the study of habitat fragmentation effects. Ecological Applications 12: 335-345.

McGarigal, K.; Cushman, S. A.; Neel, M. C. \& Ene, E. (2002): FRAGSTATS: Spatial pattern analysis program for categorical maps. Version 3. URL www.umass.edu/ landeco/research/fragstats/fragstats.html.

Microsoft Corporation (2010): Microsoft Access 2010.

Moser, B.; Jaeger, J. A. G.; TAppeiner, U.; Tasser, E. \& Eiselt, B. (2007): Modification of the effective mesh size for measuring landscape fragmentation to solve the boundary problem. Landscape Ecology 22: 447-459.

Murcia, C. (1995): Edge effects in fragmented forests - implications for conservation. Trends in Ecology E Evolution 10: 58-62.

NLfB (Niedersächsisches LANDESAMt FÜr Bodenforschung) (1997): Böden in Niedersachsen, Teil 1: Bodeneigenschaften, Bodennutzung und Bodenschutz. URL http://www.lbeg.de/extras/nlfbook/html/nds_main.htm. Retrieved 07/09/2010.

NLWKN (NIEDERSÄCHSISCHER LANDESBETRIEB FÜR WASSERWIRTSCHAFT, Küsten UND NATURschutz) (2010a): NATURA 2000: Europäische Vogelschutzgebiete und gemeldete FFH-Gebiete in Niedersachsen. URL http: //www.umwelt.niedersachsen.de/portal/live.php?navigation_id=2540\&article_ id=9124\&_psmand=10. Retrieved 09/10/2010.

NLWKN (NIEDERSÄCHSISCHER LANDESBETRIEB FÜR WASSERWIRTSCHAFT, KÜSTEN UND Naturschutz) (2010b): Naturschutzrechtlich besonders geschützte Teile von Natur und Landschaft in Niedersachsen. URL http:/ / www.umwelt.niedersachsen. de/portal/live.php?navigation_id=2543\&article_id=9065\&_psmand=10. Retrieved 03/30/2010. 
PARKs, S. (2004): ArcScript Heat Load Index. URL http://arcscripts.esri.com/details. asp?dbid=13368. Retrieved 12/18/2010.

Pilgrim, B. \& Franke, R. (1993): Kartographische Arbeitsgrundlage für faunistische und floristische Erfassungen. Naturschutz und Landschaftspflege in Niedersachsen A/5. Niedersächsisches Landesamt für Ökologie.

Schmidt, M.; KRIEbitzsch, W.-U. \& Ewald, J. (2011): Waldartenlisten der Farn- und Blütenpflanzen, Moose und Flechten Deutschlands. BfN-Skripten 299: 1-111.

Schmiedel, I.; Bergmeier, E. \& Culmsee, H. (submitted): Plant species richness patterns along a gradient of landscape modification intensity in Lower Saxony, Germany .

Schmiedel, I. \& Culmsee, H. (submitted): The influence of landscape fragmentation, expressed by the 'effective mesh size index', on regional patterns of vascular plant species richness in Lower Saxony, Germany .

Schmiedel, I.; Schacherer, A.; Hauck, M.; Schmidt, M. \& Culmsee, H. (2011): Verbreitungsmuster der Farn- und Blütenpflanzen in Niedersachsen und Bremen unter Berücksichtigung ihres Einbürgerungsstatus und ihrer Gefährdungssituation. Tuexenia 31: 211-226.

Schmiedel, I.; Schmidt, M.; Schacherer, A. \& Culmsee, H. (2013): Die Effektivität von Schutzgebieten für die Erhaltung seltener und gefährdeter Gefäßpflanzenarten: Eine Untersuchung im niedersächsischen Tiefland. Naturschutz und Landschaftsplanung 45: 45-52.

Walz, U. (2005): Landschaftszerschneidung in Grenzräumen - Sachsen und die Sächsisch-Böhmische Schweiz. GAIA 14: 171-174.

WeI, Y. \& Hoganson, H. M. (2005): Landscape impacts from valuing core area in national forest planning. Forest Ecology and Management 218: 89-106. 


\section{Patterns of vascular plant species $\mathcal{H}$ richness at the landscape scale}

Schmiedel, I.; Schacherer, A.; Hauck, M.; Schmidt, M. \& Culmsee, H. (2011): Verbreitungsmuster der Farn- und Blütenpflanzen in Niedersachsen und Bremen unter Berücksichtigung ihres Einbürgerungsstatus und ihrer Gefährdungssituation. Tuexenia 31: 211-226. 


\section{Summary}

The present study analyses the richness patterns of different groups of vascular plant species for the federal states of Lower Saxony and Bremen. For this purpose, all 1819 established species occurring in the two states as well as different sub-groups of species were considered. Sub-groups were defined by using information on residence (1509 native species, 160 archaeophytes, 145 established neophytes) and threat status (643 threatened vs. 1176 non-threatened species) of the species, respectively. Because of their contrasting demands regarding their habitats it was assumed that species belonging to the various groups would occupy different ecological niches in the landscape; therefore - on a large scale - their centres of diversity were assumed to be spatially segregated from one another.

Data on species occurrences were obtained from the floristic mapping of the two states (plant survey of Lower Saxony, NLWKN 1982-2003) and were available on a grid basis $\left(1,762\right.$ grid cells of c. $\left.30 \mathrm{~km}^{2}\right)$. The study aimed at answering the following questions: 1.) Which patterns of vascular plant species richness are observable in Lower Saxony and Bremen and the three natural landscape regions, respectively? 2.) Do the different plant species (sub-)groups show richness patterns that are distinguishable from each other and how much do their richness centers overlap? 3.) In which areas do threatened species show highest species numbers and how do these hotspots differ from that of non-threatened species?

Data were analysed by calculating the number of species per grid cell for each of the species groups mentioned above. Species richness per grid cell was then displayed using a geographical information system (GIS). To test if the centres of diversity of the species groups were overlapping, the pairwise non-parametric Spearman's rank correlation coefficient $\rho$ was calculated.

Species richness of all groups was found to be heterogeneously distributed across the states with an observable increase of species numbers from north to south and - to some extent - also from west to east. The spatial distribution of the richness centres of the different plant species groups were largely correlated with each other but also showed some interesting divergences. Considering the total number of established species occurring in the study area an aggregated distribution pattern was observable: As site conditions in the Quaternary coastal and lowland areas are relatively homogenous these regions - with exception of the major stream valleys of the Weser, Aller and Elbe - were found to be relatively species poor. In contrast, the upland area with its heterogeneous site conditions, generally hosted a high overall species richness. The large group of native species showed a distribution very similar to that of the total number of species. Archaeophytes were concentrated in the coastal area of Bremen, the Weser and Aller plains, and the loess landscapes of the lowland area, as well as smaller cities. High richness of established neophytes was mainly found in congested urban areas. Richness centres of threatened species were spatially highly differentiated: at the coast only the islands in the North Sea repre- 
sented small hotspots, while in the lowlands relatively large hotspots were situated in the Wendland, the Luneburg Heath and the Elbe-Weser triangle north of Bremen. In the uplands, the area around Göttingen, the uplands of the Weser and Leine valleys and the margins of the Harz Mountain represented well defined hotspots of Red List species. 


\section{Zusammenfassung}

Voraussetzung für die Entwicklung von Schutzstrategien für den Pflanzenartenschutz ist die Kenntnis über die Verteilung der Zentren der Artenvielfalt im Raum. Je nach Einbürgerungsstatus und Gefährdungssituation kommt verschiedenen Artengruppen dabei eine unterschiedliche Bedeutung $\mathrm{zu}$. In der vorliegenden Studie werden für die Gesamtfläche der Bundesländer Niedersachsen und Bremen die im Niedersächsischen Pflanzenarten-Erfassungsprogramm (1982-2003) auf Messtischblatt-Quadranten-Ebene erhobenen Verbreitungsdaten von Gefäßpflanzensippen unter Berücksichtigung der Gesamtflorenliste (1.819 Sippen), ihres Einbürgerungsstatus (1.509 Indigene, 160 Archäophyten, 145 etablierte Neophyten) und ihrer Gefährdungssituation (ungefährdete und gefährdete Arten; davon 643 Sippen mit Rote-Liste-Status 1, 2, 3, G oder R) ausgewertet. Auf Basis der Gesamtliste ergibt sich eine inhomogene Verteilung der Sippendichte im Gesamtuntersuchungsraum, wobei die standörtlich relativ homogene Küste sowie das Tiefland - mit Ausnahme der großen Stromtäler (Weser, Aller, Elbe) - relativ artenarm sind und das standörtlich sehr heterogene Hügel- und Bergland grundsätzlich die höchsten Sippendichten aufweist. Unter Berücksichtigung des Einbürgerungsstatus zeigen die Archäophyten jeweils die größten Überschneidungsbereiche $\mathrm{zu}$ den Indigenen und etablierten Neophyten. Die Verbreitungsmuster der großen Gruppe der Indigenen ähneln denen der Gesamtliste, während sich die Archäophyten auf den Bremer Küstenraum, das Weser-Aller-Flachland, die Börden und das südliche Weser-Leine-Bergland konzentrieren. Die Zentren der Sippenvielfalt der etablierten Neophyten liegen vor allem in städtischen Ballungsräumen und erscheinen oftmals sehr punktuell. Die Rote-Liste-Arten sind in der Mehrzahl indigen (91\%), 8\% von ihnen sind Archäo-, nur 1\% Neophyten. Ihre Diversitätszentren sind außerordentlich differenziert: An der Küste gehören nur die isoliert liegenden Nordsee-Inseln dazu, während im Tiefland das Wendland, die Lüneburger Heide und das Elbe-WeserDreieck großflächige Diversitätszentren aufweisen. Im Hügel- und Bergland finden sich vor allem im Raum Göttingen, dem Weserbergland und am Harzrand gut abgegrenzte Zentren der Rote-Liste-Artendiversität. Viele dieser bedrohten Sippen sind vermutlich Spezialisten, die an natürliche oder naturnahe Habitate angepasst und somit nur in den wenigen Landschaftsbereichen anzutreffen sind, die die entsprechenden Habitatbedingungen bieten. 


\section{Abstract}

Distribution patterns of vascular plant taxa in the federal states of Lower Saxony and Bremen, Germany, with respect to their naturalisation and threat status

Detailed knowledge about the spatial distribution of richness centres is a prerequisite for developing national strategies for the conservation of vascular plant species. Thereby, different groups of plant taxa may have different relevance depending on their naturalisation and threat status. In this study, we investigate the spatial distribution of plant species richness centres in the area of the federal states of Lower Saxony and Bremen based on data from the plant survey of Lower Saxony (Niedersächsisches Pflanzenarten-Erfassungsprogramm). Analyses include: 1.) The complete floristic list (1819 taxa) 2.) three groups of taxa defined by their naturalisation status (1509 indigenous taxa, 160 archaeophytes, 145 established neophytes), 3.) two groups of taxa defined by their threat situation (unthreatened versus vulnerable taxa, including 643 taxa which have been red listed with status 1 , 2,3 , G or R). Based on the complete floristic list, a clumped distribution pattern was observed. Site conditions in the Quaternary coastal and lowland areas are relatively homogenous and, with exception of the major stream valleys of the Weser, Aller and Elbe, relatively species poor, while the upland area, with its heterogeneous site conditions, shows the overall highest taxonomic richness. The large group of indigenous taxa shows a distribution very similar to that of the complete floristic list. Archaeophytes are concentrated in the coastal area of Bremen, the Weser and Aller plains, and the Old moraine loess landscapes, as well as smaller cities. High phytodiversity of established neophytes is mainly found in congested urban areas and several other small-scale centres of diversity. Red List species are mainly indigenous (91\%), $8 \%$ are archaeophytes and only $1 \%$ are neophytes. Their centres of phytodiversity are spatially highly differentiated: at the coast only the islands in the North Sea are small hotspots, while in the lowlands relatively large hotspots are situated in the Wendland, the Luneburg Heath and the Elbe-Weser triangle north of Bremen. In the uplands, the area around Göttingen, the uplands of the Weser and Leine valleys and the Harz mountain margins are well defined hotspots of Red List taxa. Many of these endangered species are presumably specialists adapted and limited to natural and semi-natural habitats.

Keywords: Archaeophytes, plant diversity, phytodiversity, hotspots, neophytes, Red Lists, richness centres. 


\section{Einleitung}

Das Verbreitungsmuster von Pflanzenarten im Raum wird durch deren Ausbreitungsmodi, ihr Konkurrenz- und Anpassungsverhalten, Wechselwirkungen mit der abiotischen Umwelt, durch evolutionäre und erdgeschichtliche, aber auch aktuelle, meist durch den Menschen verursachte Prozesse bestimmt (HAeuplen, 1974). Landschaftsräume mit hoher struktureller und Habitat-Heterogenität, die sich aus einem Mosaik von künstlich geschaffenen Habitaten und Resten naturnaher Vegetation zusammensetzen, weisen oftmals eine sehr hohe Pflanzenartenvielfalt auf (Deutschewitz et al., 2003). Insbesondere in städtischen Ballungszentren liegen die Artenzahlen meist höher als in dem umgebenden ländlichen Raum (HAEupler, 1974; WANIA et al., 2006; LIPPE \& KOWARIK, 2008).

Für die Erfassung von Zentren der Artenvielfalt auf Landschaftsebene kommt verschiedenen Artengruppen je nach Einbürgerungsstatus und Gefährdungssituation eine unterschiedliche Bedeutung zu. Insbesondere Diversitätszentren von indigenen und/oder gefährdeten Pflanzensippen ("Hotspots") sind für den Artenschutz von Bedeutung (Globale Strategie zur Erhaltung der Pflanzen, SeKretariat der CBD, 2007), während solche von gebietsfremden Arten, insbesondere von invasiven Neophyten, auf eine Gefährdung von heimischen Arten, ökologischen Kreisläufen und des bestehenden Landschaftsbildes hinweisen können (Huво et al., 2007). Gefährdete Arten stammen fast ausschließlich aus den Gruppen der Indigenen und Archäophyten (Definition bei WAGENITZ, 2003). In der mitteleuropäischen Landschaft sind die Hauptursachen für ihre Gefährdung die Intensivierung der Landnutzung und ihre Folgen (Eutrophierung, Entwässerung, Aufgabe extensiver Bewirtschaftungsweisen) sowie der gestiegene Flächenbedarf der Bevölkerung (GARve, 2004). Etablierte Neophyten sind dagegen unter Einfluss des Menschen seit ca. 1500 n. Chr. in ein Gebiet eingewandert und - nach zeitlichen und populationsbiologischen Etablierungskriterien (Schnittler \& Ludwig, 1996) - fester Bestandteil der Flora geworden. Aufgrund der kontrastierenden Habitatansprüche der verschiedenen Artengruppen ist anzunehmen, dass sie wie die heimischen Arten unterschiedliche Nischen in der Landschaft besetzen, so dass bei großräumiger Betrachtung ihre Zentren der Artenvielfalt räumlich getrennt sein sollten.

In dieser Studie werden für die Bundesländer Niedersachsen und Bremen die Verbreitungsmuster verschiedener Gefäßpflanzengruppen untersucht: Es werden die Gesamtheit der Arten sowie weitere, nach ihrem Einbürgerungsstatus (Indigene, Archäophyten und etablierte Neophyten) bzw. ihrer Gefährdungssituation (gefährdete und ungefährdete Arten) definierte Artengruppen betrachtet. Die Analyse umfasst die von 1982 bis 2003 erhobenen Funddaten von Sippen der Roten Liste und Florenliste der Farn- und Blütenpflanzen Niedersachsens und Bremens (GARVE, 2004) und basiert auf der floristischen Kartierung der beiden Bundesländer nach der Methodik des Niedersächsischen Pflanzenarten-Erfassungsprogramms (SCHACHERER, 2001). Dieses wird von der niedersächsischen Fachbehörde für Naturschutz (NLWKN) durchge- 
führt und liefert auf Ebene von Messtischblatt-Quadranten flächendeckende Informationen über die Funde aller Gefäßpflanzensippen.

Die vorliegende Studie ist im Rahmen des von der Deutschen Bundesstiftung Umwelt geförderten Projektes "Identifizierung von Indikatorartengruppen für ein Biodiversitäts-Monitoring zur Bewertung von Grünland- und Waldlebensräumen" entstanden. Sie stellt eine grundlegende Auswertung des Pflanzenartendatensatzes dar, auf der weitere, speziellere Untersuchungen aufbauen. Auf Grundlage der Pflanzenartendaten sollen folgende Fragestellungen untersucht werden:

1. Welche Diversitätsmuster sind im gesamten Gebiet bzw. in den drei Naturräumen Küste, Tiefland sowie Hügel- und Bergland auf Grundlage der Gesamtliste der Farn- und Blütenpflanzen erkennbar?

2. Ergeben sich für die verschiedenen Gruppen je nach Einbürgerungsstatus unterschiedliche Muster der Phytodiversität und wie stark überschneiden sich die Zentren ihrer Diversität?

3. Wo befinden sich die Zentren der Sippenvielfalt der Rote-Liste-Arten (Hotspots im Sinne von Schmitt \& Haeupler, 2009) und wie unterscheiden sie sich von den Diversitätszentren der ungefährdeten Sippen?

\section{Material und Methoden}

\section{Untersuchungsgebiet}

Das Untersuchungsgebiet umfasst Niedersachsen und Bremen, wobei Niedersachsen mit einer Größe von $47.624 \mathrm{~km}^{2}$ das zweitgrößte, Bremen mit einer Größe von $404 \mathrm{~km}^{2}$ das kleinste Bundesland Deutschlands ist (Niedersachsen, 2008). Das Gebiet wird in drei Großregionen unterteilt: die Küste, das Tiefland und das Hügel- und Bergland (Abb. 3.1).

Die einzelnen Regionen unterscheiden sich stark in ihrer Naturraumausstattung: Die Küste wird geologisch vor allem durch marine Ablagerungen aus dem Holozän bestimmt (Heunisch et al., 2007). Ihr Klima ist ozeanisch geprägt, der mittlere Jahresniederschlag beträgt $700-900 \mathrm{~mm}$, die mittlere Jahrestemperatur liegt bei durchschnittlich $9^{\circ} \mathrm{C}$. Vorherrschende Bodentypen sind Marschen, Syroseme, podsolige Ranker und Gleye (NLFB, 1997). Die Küstenregion wird dominiert von Grünländern, die etwa $57 \%$ der Region einnehmen. Während Ackerflächen hier einen Anteil von $22 \%$ besitzen, sind lediglich $1 \%$ Wälder vorhanden.

Das niedersächsische Tiefland wird von pleistozänen Ablagerungen dominiert (Heunisch et al., 2007). Von West nach Ost bringt ein Kontinentalitätsgradient Jahresniederschlagsmittel von $800 \mathrm{~mm}$ im Westen bis etwa $550 \mathrm{~mm}$ im Osten sowie Jahresmitteltemperaturen von $8.0-8.5^{\circ} \mathrm{C}$ mit sich. Entlang dieses Klimagradienten sind unterschiedliche Böden ausgebildet: Während im ozeanischen Westen von Sickerwasserbildung, Vernässung und Stoffverlagerung geprägte Böden (Moorböden, Podsole) vorherrschen, finden sich im subkontinentalen Osten vor allem Braunerden 
und Ranker (NLfB, 1997). Das Tiefland wird dominiert von Ackerflächen (41\%), Wälder und Grünländer nehmen jeweils etwa knapp 1/4 der Region ein (21 bzw. $22 \%)$.

Das standörtlich sehr heterogene Hügel- und Bergland wird vom Meso- und Paläozoikum (Heunisch et al., 2007) dominiert und weist aufgrund der stark variierenden Topographie besonders unterschiedliche klimatische Verhältnisse auf. Das Bergvorland ist geprägt von Löss- und Sandlössablagerungen sowie Ton-, Sand- und Kalkgesteinen (NLFB, 1997). Neben Parabraunerden und Braunerden kommen auch Übergänge zu Pseudogleyen vor (Bördenvorland). In der Lössbörde treten großflächig Schwarz- und Parabraunerden auf. Die Jahresdurchschnittstemperatur der tieferen Lagen (Becken) liegt $1-2^{\circ} \mathrm{C}$ über der der Höhenzüge; die mittleren Jahresniederschläge sind auf den Höhenzügen deutlich höher $(800-900 \mathrm{~mm})$ als in den Beckenlagen (650-750 mm). Der Harz im Südosten Niedersachsens nimmt eine Sonderstellung ein: Der Hochharz (in Niedersachsen bis $971 \mathrm{~m}$ ü. NN) weist mittlere Jahresniederschläge von bis zu $1.500 \mathrm{~mm}$ und eine Jahresmitteltemperatur von nur $7^{\circ} \mathrm{C}$ sowie lange, schneereiche Winter auf und ist somit (hoch-)montan geprägt (NLFB, 1997). Sowohl im Hochharz als auch im Oberharz, der submontane bis montane Klimaverhältnisse aufweist, kommen basenarme Silikatgesteine sowie Tonschiefer und Grauwacken vor. Vorherrschende Bodentypen sind hier Braunerden und Podsole (NLFB, 1997). Die Region weist von den drei Großregionen den höchsten prozentualen Waldanteil auf (32\%). Grünländer nehmen hier nur knapp 9\% der Fläche ein, während $45 \%$ von Ackerflächen geprägt sind.

\section{Untersuchungsraster}

Das Untersuchungsgebiet ist auf Grundlage der Topographischen Karte 1:25.000 in ein Raster von Messtischblatt-Quadranten (MTB-Quadranten, im Folgenden nur Quadranten genannt) mit einer Größe von etwa $5.5 \mathrm{~km} \times 5.5 \mathrm{~km}$ gegliedert. Für die vorliegenden Analysen wurden 1.762 Quadranten berücksichtigt, von diesen liegen 1.386 vollständig im Untersuchungsgebiet und sind gänzlich von Land bedeckt (Vollquadranten), weitere 376 liegen teilweise oder vollständig im Meer oder teilweise an der Grenze zu anderen Bundesländern (Randquadranten). Es wurden 255 Quadranten der Küste, 1.101 Quadranten dem Tiefland und 406 Quadranten dem Hügel- und Bergland zugeordnet. Dabei wurden Quadranten im Grenzgebiet zweier Regionen jeweils der Region zugeordnet, die den größeren Flächenanteil hatte.

\section{Pflanzenartendaten}

Die Gesamtliste der Farn- und Blütenpflanzen in Niedersachsen und Bremen richtet sich nach GARVE (2004). Verbreitungsangaben auf Quadranten-Ebene wurden der Datenbank des Niedersächsischen Pflanzenarten-Erfassungsprogramms entnommen (NLWKN 1982-2003, Stand 15.12.2008). Eine Sammlung von Artenverbreitungskar- 


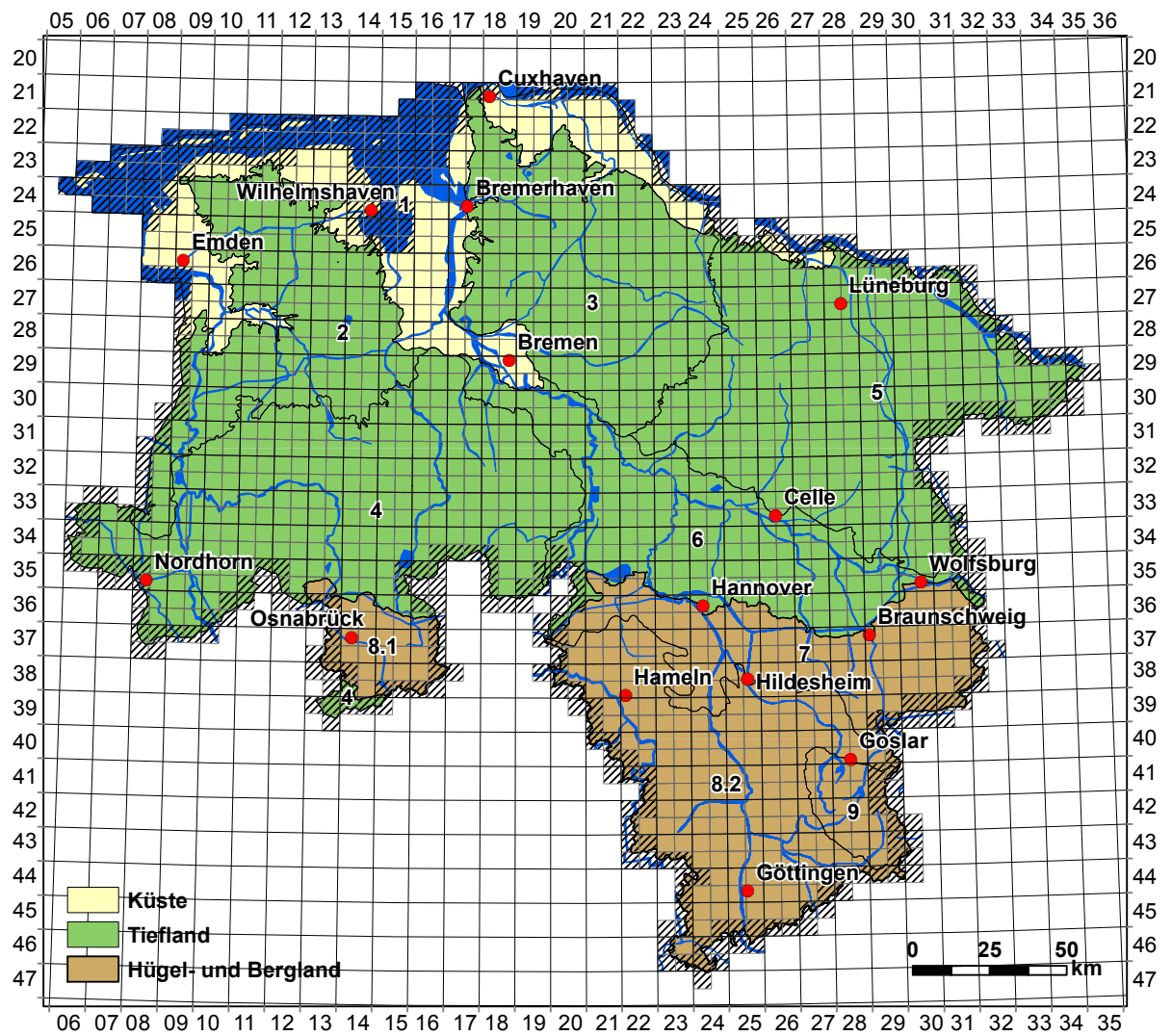

Abbildung 3.1: Das Untersuchungsgebiet umfasst die Gesamtfläche der Bundesländer Niedersachsen und Bremen und wird in drei naturräumliche Großregionen unterteilt: die Küste, das Tiefland und das Hügel-/Bergland. Eine weitere Untergliederung erfolgt in zehn naturräumliche Hauptregionen ( 1 = Watten und Marschen, 2 = Ostfriesisch-Oldenburgische Geest, 3 = Stader Geest, 4 = Ems-Hunte-Geest und Dümmer-Geestniederung, $5=$ Lüneburger Heide und Wendland, 6 = Weser-Aller-Flachland, 7 = Börden, 8.1 = Osnabrücker Hügelland, 8.2 = Weser- und Leinebergland, 9 = Harz). Die 1.762 Messtischblatt-Quadranten sind grundlegende Kartiereinheit des Niedersächsischen Pflanzenarten-Erfassungsprogramms (Zahlenangaben am Rand entsprechen der TK25-Basisblatt-Nummer). Schraffierte Quadranten wurden auf einer kleineren Fläche floristisch erfasst, da sie teilweise oder gänzlich im Meer bzw. in angrenzenden Bundesländern liegen.

Figure 3.1: The study area the federal states of Lower Saxony and Bremen, with its three natural landscape regions (Küste, coast; Tiefland, lowlands; Hügel- und Bergland, uplands). The region can be further divided into ten natural regions $(1=$ Wadden Sea and marshes, $2=$ East Frisian and Oldenburg moraine lowlands, $3=$ Stade moraine lowlands, $4=$ Ems, Hunte and Dümmer moraine lowlands, $5=$ Luneburg Heath and Wendland, $6=$ Weser and Aller plains, $7=$ Old moraine loess landscapes, $8.1=$ Uplands of Osnabrück, $8.2=$ Uplands of the Weser and Leine valleys, $9=$ Harz mountains.) Plant occurrence data from the plant survey of Lower Saxony were available for 1762 quadrants of the topographical map (scale 1:25,000, i. e. about $5.5 \times 5.5 \mathrm{~km}$ in size, frame numbers equal ordnance map numbers). The area of hatched quadrants has only been partly inventoried, because they are either only partially covered by land area or are situated in neighbouring federal states. 
ten mit Angaben zur Funddichte wurde von GARve (2007) veröffentlicht. Eine frühere Auswertung der Anzahl der Rote-Liste-Arten je Quadrant erfolgte durch GARve (1994).

Der Pflanzenartendatensatz wurde nach folgenden Kriterien gruppiert:

1. Gesamtliste: alle Sippen der Farn- und Blütenpflanzen mit floristischem Normalstatus, d. h. Indigene, Archäophyten und etablierte Neophyten, unter Ausschluss von unbeständigen und synanthropen Vorkommen sowie Sippen, die in Niedersachsen und Bremen ausgestorben oder verschollen sind (1.819 Sippen mit Einträgen in der Datenbank).

2. Einbürgerungsstatus: Indigene (1.509 Sippen), Archäophyten (160 Sippen) bzw. etablierte Neophyten (145 Sippen) nach der von GARve (2004) verwendeten Definition. Der Einbürgerungsstatus der Sippen wurde der Niedersächsischen Florenliste entnommen, wobei der Etablierungsstatus der Neophyten von GARve (2004) übernommen wurde. Die Unterscheidung von Indigenen und Archäophyten erfolgte durch Abgleich mit der BIOLFLOR-Datenbank (KLOTz et al., 2002). Für fünf der den Indigenen/Archäophyten zugeordneten Sippen konnte kein Einbürgerungsstatus festgestellt werden (Panicum riparium, Persicaria mitis, Potamogeton $\mathrm{x}$ undulatus, Rumex $\mathrm{x}$ pratensis, Silene x hampeana).

3. Gefährdungssituation: ungefährdete Sippen inklusive der Arten der Vorwarnliste (1.176 Sippen) bzw. gefährdete Sippen (643 Sippen mit Rote-Liste-Status 1, 2, 3, G oder R), wobei sich der Gefährdungsstatus auf das Gesamtgebiet Niedersachsen und Bremen bezieht (GARVE, 2004).

\section{Datenanalyse}

Für jede der untersuchten Gruppen wurde die Sippenzahl je Raumeinheit (Quadrant) erfasst und mithilfe eines Geographischen Informationssystems dargestellt (ArcGISArcInfo 9.2; ESRI INC., 2007). Bei der Visualisierung der Verbreitungsmuster wurden natürliche Grenzwerte verwendet, wobei jeweils fünf Sippenzahl-Klassen gebildet wurden. In den Abbildungen wurden negative Abweichungen vom Mittelwert mit abgestuften Grautönen und die Klassen um bzw. oberhalb des Mittelwerts in Farbabstufungen dargestellt.

Zusätzlich wurden die mittleren und maximalen Sippenzahlen je Gruppe und Quadrant für Niedersachsen/Bremen bzw. die einzelnen Regionen Küste, Tiefland und Hügel- und Bergland ermittelt. Räumliche Autokorrelationen von Quadranten mit hohen Sippenzahlen (Klumpungen der Diversitätszentren) wurden als Moran's Index berechnet, wobei Werte gegen -1 auf eine starke Streuung und Werte gegen +1 auf eine starke Klumpung von Quadranten mit hoher Artenvielfalt im Raum hinweisen.

Für die nach ihrem floristischen Status (Indigene, Archäophyten, etablierte Neophyten) bzw. ihrem Gefährdungsstatus (ungefährdete Arten, Rote-Liste-Arten) gruppierten Sippen wurde jeweils für Paare dieser drei bzw. zwei Gruppen mittels des Rangkorrelationskoeffzienten $\rho$ nach Spearman für nicht normalverteilte Daten 
(Shapiro-Wilk Normality-Tests) mit der R-Software (R Development CoRe TeAm, 2012) überprüft, ob ihre Diversitätswerte (Zentren der Sippen-Diversität) miteinander korreliert sind. Der Koeffizient $\rho$ kann Werte zwischen -1 und +1 annehmen, je nachdem, ob eine negative, positive bzw. - bei einer Annäherung gegen Null - keine Korrelation vorhanden ist.

\section{Ergebnisse}

\section{Muster in der Phytodiversität der Gefäßpflanzen: Sippenzahlen auf Basis der gesamten Florenliste}

Von den insgesamt 1.819 in Niedersachsen und Bremen vorkommenden Gefäßpflanzensippen mit floristischem Normalstatus kommen im Mittel etwa ein Viertel (435 Sippen) je Quadrant vor (Tab. 3.1). Maximal wurden 747 Sippen innerhalb eines Quadranten gezählt. Tabelle 3.2 listet die fünf Quadranten mit den höchsten Sippenzahlen auf Basis der gesamten Florenliste auf. Die sippenreichsten Quadranten kommen verstreut vor. Phytodiversitätszentren lassen sich im südlichen und östlichen Niedersachsen sowie nordwestlich von Bremen identifizieren (Abb. 3.2). Vor allem im nördlichen und westlichen Niedersachsen ist die Gefäßpflanzendichte gering. Die deutliche räumliche Konzentration von Diversitätszentren kann auch durch die starke räumliche Autokorrelation belegt werden (Tab. 3.3).

Vergleicht man die Gefäßpflanzenvielfalt der drei naturräumlichen Großregionen, so weist das Berg- und Hügelland sowohl die höchste absolute als auch die höchste mittlere Sippenzahl je Quadrant auf (Tab. 3.1). Die Sippenzahl ist in allen Quadranten relativ gleichmäßig hoch, eine Ausnahme stellt hier nur der Solling dar. Das Tiefland nimmt eine Mittelstellung ein: Die mittlere Sippenzahl je Quadrant entspricht in etwa dem für die Gesamtfläche ermittelten Durchschnitt. Während das westliche Tiefland relativ artenarm ist, finden sich Zentren der Sippen-Vielfalt vor allem im Weser-AllerFlachland - insbesondere die Flusstäler von Aller, Leine und Weser zeichnen sich deutlich ab -, in der Lüneburger Heide und im Wendland. An der Küste kommen im Mittel nur 306 Sippen je Quadrant vor, so dass die Sippenzahl wesentlich unter dem Landesdurchschnitt liegt. Lediglich im Raum Bremen und auf den Inseln sind relativ hohe Sippenzahlen je Quadrant zu verzeichnen. 


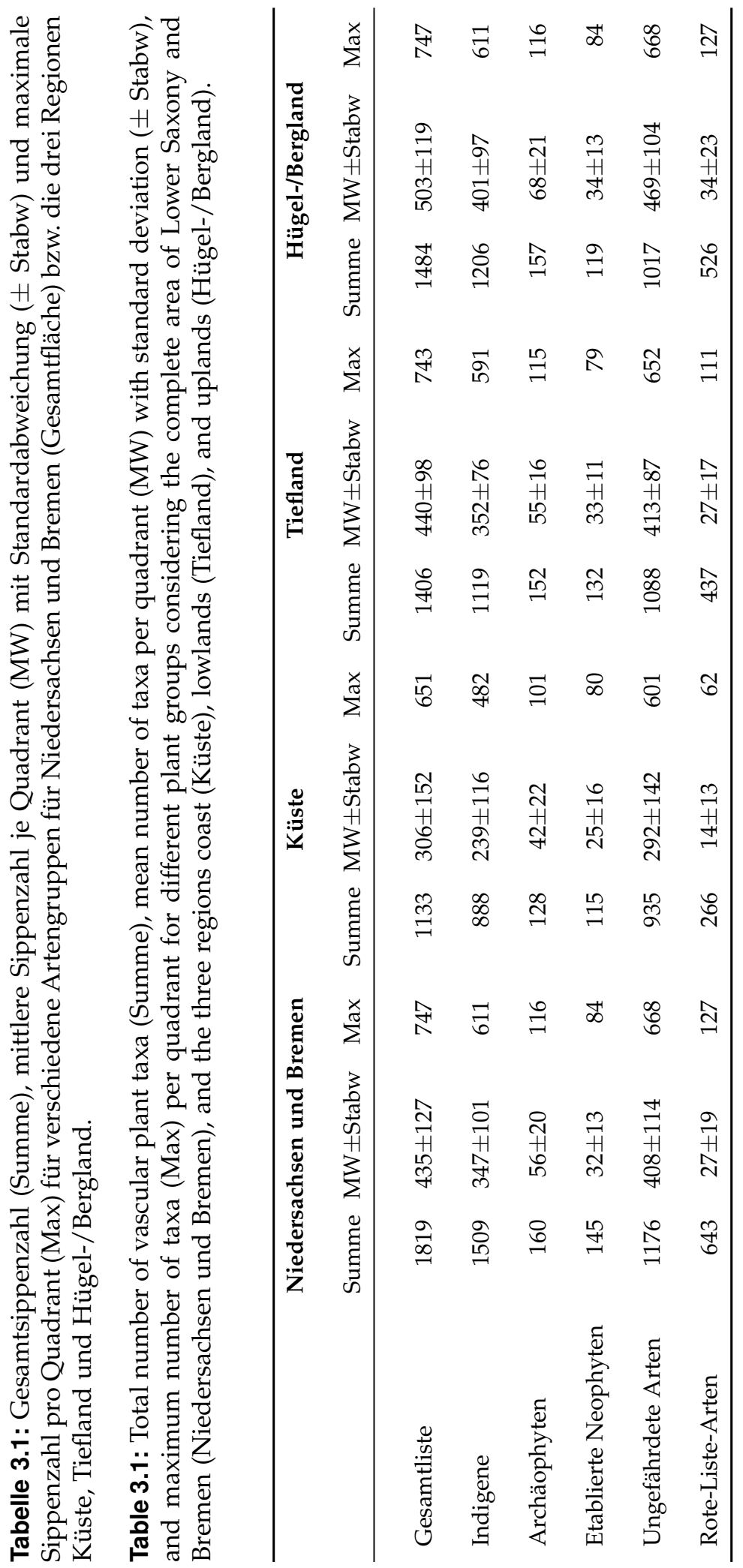




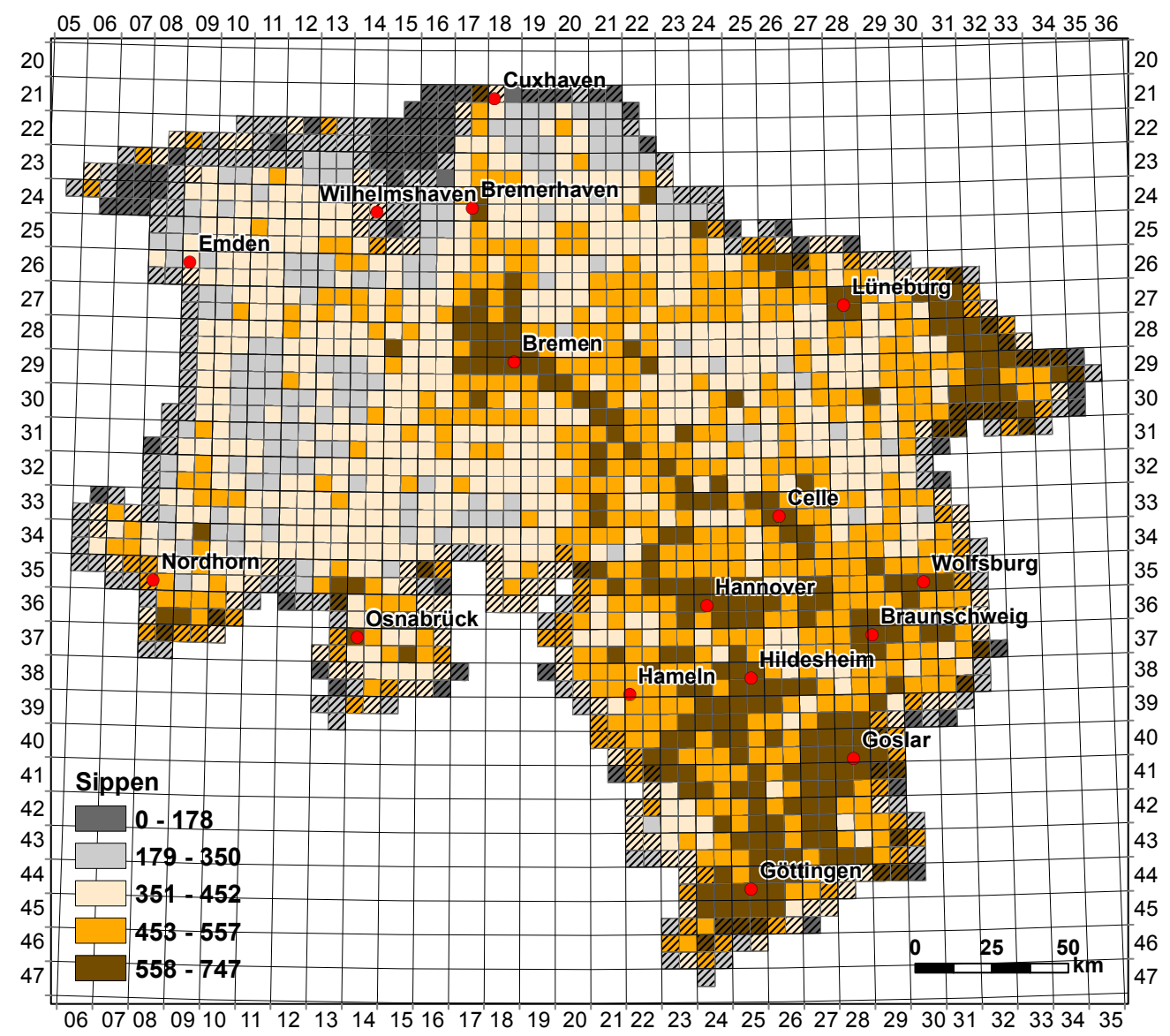

Abbildung 3.2: Anzahl der in Niedersachsen und Bremen vorkommenden Gefäßpflanzensippen mit floristischem Normalstatus je Quadrant basierend auf der Gesamtliste (1.819 Sippen).

Figure 3.2: Number of taxa (excluding erratic and synanthropic occurences) per quadrant in Lower Saxony and Bremen, considering the complete list of 1819 vascular plant taxa.

\section{Muster in der Phytodiversität der Gefäßpflanzen unter Berücksichtigung ihres Einbürgerungsstatus}

Die absolute Zahl der Indigenen ist mit 1.509 Sippen um ein Zehnfaches höher als die der Archäophyten (160 Arten) und der etablierten Neophyten (145 Arten) (Tab. 3.1). Dementsprechend unterscheiden sich auch die mittleren Sippenzahlen zwischen den Gruppen mit durchschnittlich 347 Indigenen, 56 Archäophyten und 32 etablierten Neophyten je Quadrant. An der Küste kommen in allen Gruppen weniger Sippen vor als im Tiefland bzw. Hügel- und Bergland, wobei letztere Region - jeweils nur mit geringem Unterschied zum Tiefland - etwas artenreicher ist. Lediglich die absolute Zahl der etablierten Neophyten ist im Tiefland höher als in den übrigen Regionen. 
Tabelle 3.2: Auflistung der jeweils fünf sippenreichsten Quadranten je Artengruppe . Quadrant $=$ Nummer das Quadranten, Sippen = Anzahl der Sippen innerhalb des Quadranten, Ort $=$ Innerhalb des Quadranten gelegene Stadt/ Ortschaft.

Table 3.2: A list of the five quadrants with the highest number of taxa per species group. Quadrant $=$ Number of the quadrant, Sippen $=$ Number of taxa inside the quadrant, Ort $=$ City/Town located inside the quadrant.

\begin{tabular}{|c|c|c|c|c|c|c|}
\hline Artengruppe & & 1 & 2 & 3 & 4 & 5 \\
\hline Gesamtliste & $\begin{array}{l}\text { Quadrant } \\
\text { Sippen } \\
\text { Ort }\end{array}$ & $\begin{array}{c}4028 / 3 \\
747 \\
\text { Langelsheim }\end{array}$ & $\begin{array}{c}2832 / 1 \\
743 \\
\text { Hitzacker }\end{array}$ & $\begin{array}{c}3729 / 2 \\
736 \\
\text { Weddel }\end{array}$ & $\begin{array}{c}4129 / 1 \\
727 \\
\text { Bad Harzburg }\end{array}$ & $\begin{array}{l}3032 / 3 \\
726 \\
\text { Luckau }\end{array}$ \\
\hline Indigene & $\begin{array}{l}\text { Quadrant } \\
\text { Sippen } \\
\text { Ort }\end{array}$ & $\begin{array}{c}4429 / 2 \\
611 \\
\text { Walkenried }\end{array}$ & $\begin{array}{c}4028 / 3 \\
598 \\
\text { Langelsheim }\end{array}$ & $\begin{array}{l}3032 / 3 \\
591 \\
\text { Luckau }\end{array}$ & $\begin{array}{c}4129 / 1 \\
590 \\
\text { Bad Harzburg }\end{array}$ & $\begin{array}{c}2832 / 1 \\
586 \\
\text { Hitzacker }\end{array}$ \\
\hline Archäophyten & $\begin{array}{l}\text { Quadrant } \\
\text { Sippen } \\
\text { Ort }\end{array}$ & $\begin{array}{c}3624 / 4 \\
116 \\
\text { Laatzen }\end{array}$ & $\begin{array}{c}3624 / 1 \\
115 \\
\text { Hannover }\end{array}$ & $\begin{array}{c}3625 / 1 \\
114 \\
\text { Misburg }\end{array}$ & $\begin{array}{c}3729 / 1 \\
114 \\
\text { Braunschweig }\end{array}$ & $\begin{array}{c}3825 / 2 \\
108 \\
\text { Hildesheim }\end{array}$ \\
\hline $\begin{array}{l}\text { Etablierte } \\
\text { Neophyten }\end{array}$ & $\begin{array}{l}\text { Quadrant } \\
\text { Sippen } \\
\text { Ort }\end{array}$ & $\begin{array}{c}3729 / 1 \\
84 \\
\text { Braunschweig }\end{array}$ & $\begin{array}{c}2818 / 4 \\
80 \\
\text { Gröpelingen }\end{array}$ & $\begin{array}{c}2918 / 2 \\
80 \\
\text { Bremen }\end{array}$ & $\begin{array}{c}3624 / 1 \\
79 \\
\text { Hannover }\end{array}$ & $\begin{array}{c}2818 / 3 \\
76 \\
\text { Grambke }\end{array}$ \\
\hline $\begin{array}{l}\text { Ungefährdete } \\
\text { Arten }\end{array}$ & $\begin{array}{l}\text { Quadrant } \\
\text { Sippen } \\
\text { Ort }\end{array}$ & $\begin{array}{c}3729 / 1 \\
\quad 668 \\
\text { Braunschweig }\end{array}$ & $\begin{array}{c}3729 / 2 \\
663 \\
\text { Weddel }\end{array}$ & $\begin{array}{c}4129 / 1 \\
655 \\
\text { Bad } \\
\text { Harzburg }\end{array}$ & $\begin{array}{c}4028 / 3 \\
654 \\
\text { Langelsheim }\end{array}$ & $\begin{array}{l}3032 / 3 \\
652 \\
\text { Luckau }\end{array}$ \\
\hline $\begin{array}{l}\text { Rote-Liste- } \\
\text { Arten }\end{array}$ & $\begin{array}{l}\text { Quadrant } \\
\text { Sippen } \\
\text { Ort }\end{array}$ & $\begin{array}{c}4429 / 2 \\
127 \\
\text { Walkenried }\end{array}$ & $\begin{array}{l}2934 / 4 \\
111 \\
\text { Gartow }\end{array}$ & $\begin{array}{c}2832 / 1 \\
110 \\
\text { Hitzacker }\end{array}$ & $\begin{array}{c}4429 / 1 \\
109 \\
\text { Bad Sachsa }\end{array}$ & $\begin{array}{c}2731 / 4 \\
108 \\
\text { Glienitz }\end{array}$ \\
\hline
\end{tabular}

Die Phytodiversitätskarte der indigenen Sippen (Abb. 3.3) weist starke Parallelen zur vorher besprochenen Darstellung der Sippen der gesamten Florenliste (Abb. 3.2) auf. Im Vergleich der drei Regionen ist die Sippenzahl je Quadrant im Hügel- und Bergland meist überdurchschnittlich hoch. Lediglich im Solling und in den Börden entsprechen die Sippenzahlen etwa dem Durchschnitt. Im Tiefland sind insbesondere weite Teile der Ems-Hunte-Geest und Dümmer-Geestniederung sehr artenarm. Das Wendland, Teile der Stader Geest und des Weser-Aller-Flachlandes - insbesondere wieder das Aller- und Leinetal - sind dagegen ausgesprochene Diversitätszentren. Mit Ausnahme des Raumes Bremen ist die Küstenregion nahezu flächendeckend arm an Indigenen. Die fünf sippenreichsten Quadranten (Tab. 3.2) sind anders gelagert als die der Gesamtliste, weisen jedoch wie diese ebenfalls eine recht zerstreute Verteilung auf. 
Tabelle 3.3: Moran's Index als Maß für die räumliche Autokorrelation der Phytodiversitätszentren für die verschiedenen Gruppen.

Table 3.3: Moran's Index as a measure for spatial autocorrelation of plant richness centres considering all plant groups.

\begin{tabular}{lccc}
\hline Artengruppe & Moran's Index & Z-Wert & p-Wert \\
\hline Gesamtliste & 0,138 & 148,68 & 0,01 \\
$\quad$ Region Küste & 0,157 & 26,04 & 0,01 \\
$\quad$ Region Tiefland & 0,110 & 75,25 & 0,01 \\
$\quad$ Region Hügel-/Bergland & 0,062 & 18,30 & 0,01 \\
& & & \\
Indigene & 0,133 & 143,18 & 0,01 \\
Archäophyten & 0,166 & 178,18 & 0,01 \\
Etablierte Neophyten & 0,090 & 97,69 & 0,01 \\
Ungefährdete Arten & 0,142 & 152,39 & 0,01 \\
Rote-Liste-Arten & 0,097 & 104,77 & 0,01 \\
\hline
\end{tabular}

Ein differenzierteres Muster zeigt die Phytodiversitätskarte der Archäophyten (Abb. 3.4). Hier sind insbesondere der Bremer Küstenraum, das Weser-AllerFlachland, die Börden sowie das südliche Weser-Leine-Bergland ausgesprochene Phytodiversitätszentren. Diese überschneiden sich stark mit denen der indigenen Sippen (Tab. 3.4), treten aber noch stärker räumlich konzentriert auf (Tab. 3.3). Dies zeigt sich auch bei der Betrachtung der fünf sippenreichsten Quadranten (Tab. 3.2): Die drei artenreichsten Quadranten konzentrieren sich im Raum Hannover, weiterhin ergeben sich hohe Sippenzahlen um Braunschweig und Hildesheim.

Die Phytodiversitätskarte der etablierten Neophyten (Abb. 3.5) zeigt zum einen eine großflächige Konzentration im Bremer Küstenraum, dem Weser-Aller-Flachland und den Börden, zum anderen aber auch eine Vielzahl von zum Teil sehr isolierten Einzelquadranten mit überdurchschnittlich hohen Sippenzahlen. Dabei sind städtische Ballungsräume (Großstädte $\geq 100.000$ Einwohnern; HeineberG, 2006) grundsätzlich Zentren der Neophyten-Diversität. Diese starke Streuung von großflächigen und punktuellen Zentren der Neophyten-Diversität führt zu einem relativ geringen räumlichen Klumpungseffekt (Tab. 3.3). Dies spiegelt sich auch in der zerstreuten Verteilung der fünf sippenreichsten Quadranten wider (Tab. 3.2). Gleichzeitig ist jedoch der räumliche Überschneidungsbereich zwischen Archäophyten und etablierten Neophyten sehr groß und auch größer als der zwischen Indigenen und Archäophyten bzw. Indigenen und Neophyten (Tab. 3.4).

\section{Muster in der Phytodiversität der Gefäßpflanzen unter Berücksichtigung ihrer Gefährdungssituation}

Etwa ein Drittel der Gefäßpflanzen der Gesamtflorenliste (634 Sippen) sind als selten oder gefährdet eingestuft (Tab. 3.1). Für diese Gruppe werden im Hügel- und Berg- 
Tabelle 3.4: Ähnlichkeiten in den Verbreitungsschwerpunkten der Gruppen nach Einbürgerungsstatus bzw. Gefährdungssituation. Ergebnisse der Berechnung des Rangkorrelationskoeffzienten $\rho$ nach Spearman. ${ }^{* * *}$, signifikant auf dem Niveau $\mathrm{p} \leq 0,001$.

Table 3.4: Similarities in the distribution of species groups sorted by naturalization status (indigenous, archaeophytes, established neophytes) and threat situation (non-threatened vs. Red List taxa). Results based on the calculation of Spearman's Rank Correlation Coefficient $\rho{ }^{* * *}$, significant with $\mathrm{p} \leq 0.001$.

\begin{tabular}{lrc}
\hline & Spearmans $\rho$ & p-Wert \\
\hline Indigene - Archäophyten & $0,744^{* * *}$ & $<0,001$ \\
Indigene - etablierte Neophyten & $0,681^{* * *}$ & $<0,001$ \\
Archäophyten - etablierte Neophyten & $0,805^{* * *}$ & $<0,001$ \\
Rote-Liste-Arten - ungefährdete Sippen & $0,691^{* * *}$ & $<0,001$ \\
\hline
\end{tabular}

land sowohl die höchsten absoluten als auch mittleren Zahlen pro Quadrant erreicht. Die Mehrzahl der Rote-Liste-Arten gehört der Gruppe der Indigenen an, jedoch sind auch fast ein Drittel der Archäophyten Rote-Liste-Arten, während nur $6 \%$ der Neophyten als gefährdet eingestuft werden (Tab. 3.5).

Die räumlichen Phytodiversitätsmuster der ungefährdeten Sippen (Abb. 3.6) weichen von denen der Rote-Liste-Arten (Abb. 3.7) stark ab, wobei der Überschneidungsbereich aber dennoch relativ hoch bleibt (Tab. 3.4). Insgesamt sind die Rote-ListeArten stärker auf mehrere, kleinflächigere Raumeinheiten verstreut als die stark zusammenhängenden Zentren der ungefährdeten Arten (Tab. 3.3). In der insgesamt eher artenarmen Küstenregion bilden nur die Nordsee-Inseln isolierte Hotspots der RoteListe-Arten (zum Hotspot-Begriff siehe Schmitt \& HAEupler, 2009). Im Tiefland ist die Überlagerung von Verbreitungsmustern der ungefährdeten und gefährdeten Sippen relativ hoch, wobei insbesondere das Wendland (vgl. Tab. 3.2), die Lüneburger Heide und das Elbe-Weser-Dreieck nördlich von Bremen Hotspots der Rote-ListeArten sind. Während im Hügel- und Bergland die Anzahl der ungefährdeten Sippen fast überall überdurchschnittlich hoch ist, weisen die Rote-Liste-Arten dagegen stark abgegrenzte Hotspots im Raum Göttingen, dem Weserbergland und am Harzrand (vgl. Tab. 3.2) auf.

\section{Diskussion}

Die räumliche Verteilung der Pflanzensippenvielfalt in Niedersachsen und Bremen weist für alle betrachteten Gruppen sowohl von Nord nach Süd als auch in gewissem Maße von West nach Ost eine Zunahme in der Sippendichte auf. Dabei ist das Hügel- und Bergland grundsätzlich die Region mit der höchsten Sippendichte. Eine Ausnahme bilden hier nur die Neophyten. 


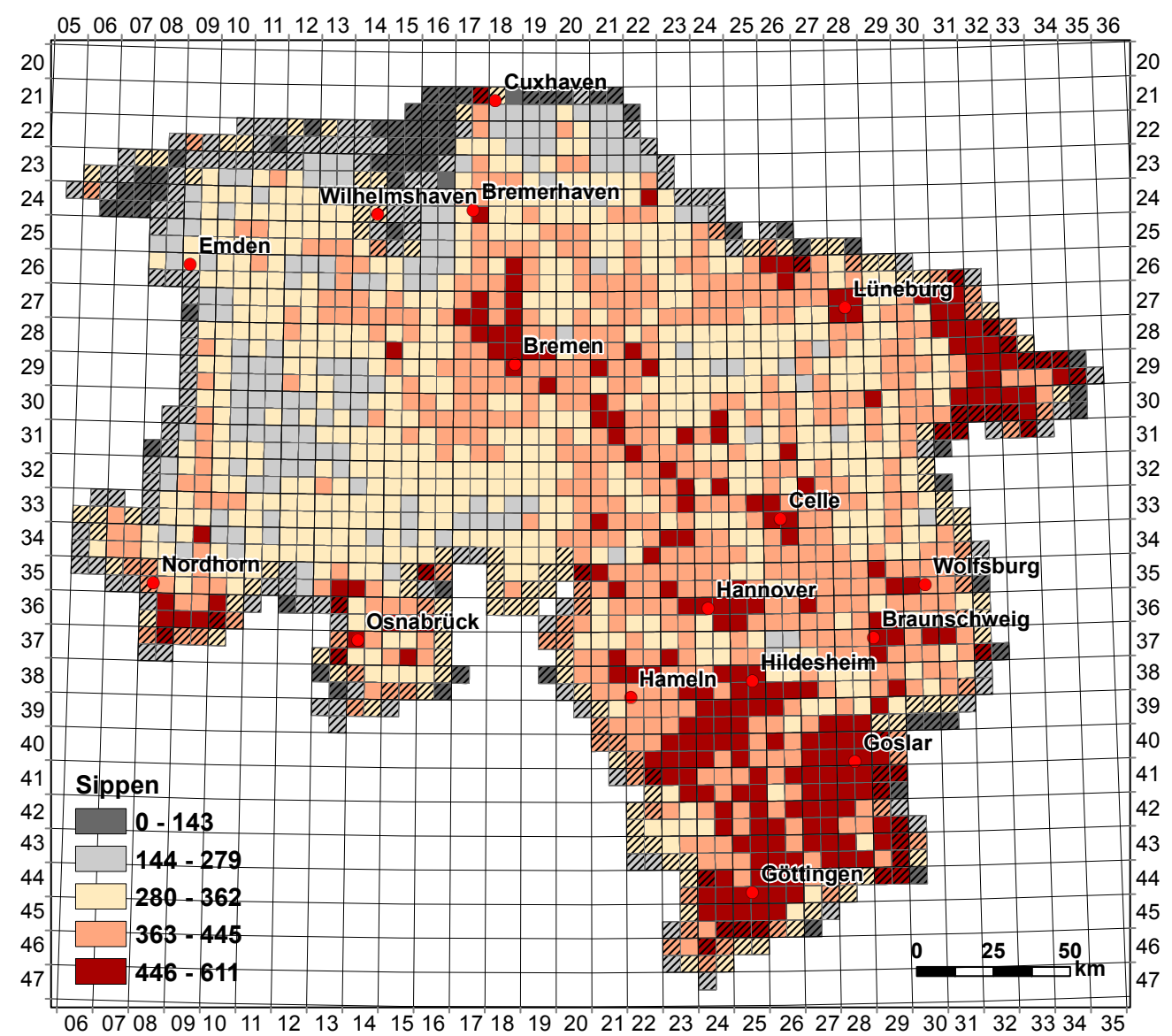

Abbildung 3.3: Anzahl der in Niedersachsen und Bremen vorkommenden 1.509 indigenen Gefäßpflanzensippen.

Figure 3.3: Number of taxa per quadrant in Lower Saxony and Bremen, considering 1509 indigenous vascular plant taxa.

Die relative Artenarmut des norddeutschen Tieflandes ist sicherlich u. a. dadurch bedingt, dass die landschaftliche Heterogenität (Klima, Relief, Gesteine, Böden) des Hügel- und Berglandes von den Lössbörden bis in den Hochharz wesentlich höher ist als die des relativ uniform vom Quartär geprägten Tieflandes. Die kleinräumigen ökologischen Gradienten dürften somit zusätzlich sowohl eine höhere absolute Sippenzahl als auch höhere Sippendichten im südlichen Niedersachsen bedingen. Das insbesondere im Tiefland ausgeprägte Ost-West-Gefälle verläuft parallel zum klimatischen subkontinental-ozeanischen Gradienten. Insbesondere im nördlichen Harzvorland und im Wendland liegen die westlichen Verbreitungsgrenzen vieler Gefäßpflanzenarten, die Vorposten einer östlich der Elbe reicheren, subkontinental getönten Flora sind. 


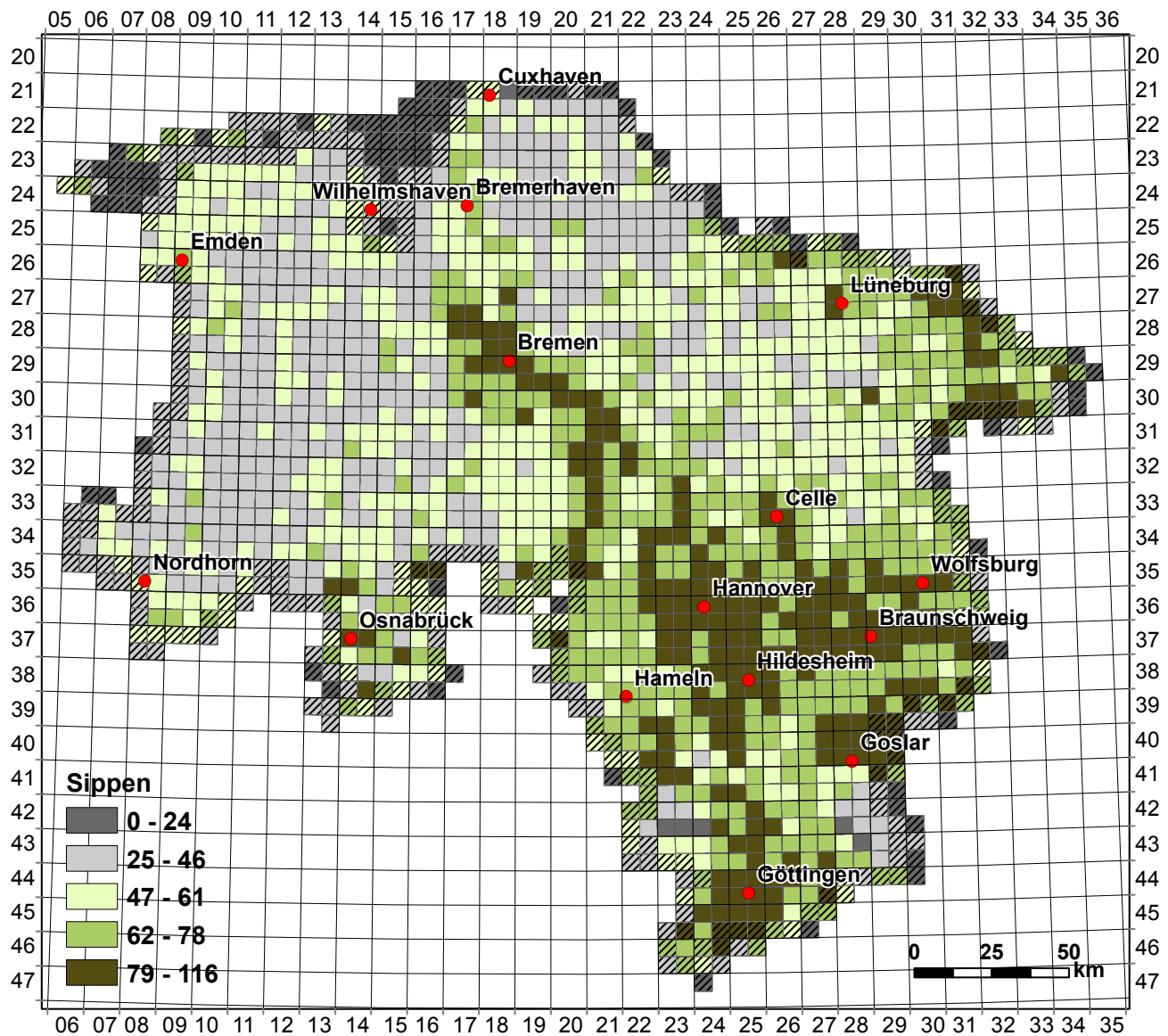

Abbildung 3.4: Sippenzahl je Quadrant der in Niedersachsen und Bremen vorkommenden 160 Archäophyten.

Figure 3.4: Number of taxa per quadrant in Lower Saxony and Bremen, considering 160 archaeophytes.

Die Verbreitungsmuster der Artengruppen nach Einbürgerungsstatus weisen große räumliche Überschneidungen auf. Während die Lage der Diversitätszentren von Indigenen und etablierten Neophyten im Tiefland viele Parallelen aufweist, sind sie im Hügel- und Bergland überwiegend negativ korreliert. KüHN et al. (2003) zeigten in einer deutschlandweiten Analyse, dass die geologische Heterogenität eines Gebietes der wichtigste Faktor für die Sippenzahlen sowohl der Einheimischen als auch der Gebietsfremden ist. PYšEK et al. (2002) erklärten die positive Korrelation von Indigenen und Neophyten in Naturschutzgebieten Tschechiens mit einer hohen Habitatdiversität in den entsprechenden Gebieten, die dazu führt, dass die beiden Gruppen nicht unbedingt miteinander in Konkurrenz stehen müssen. Dies mag auch für die positiv korrelierenden Vorkommen im niedersächsischen Tiefland zutreffen. Gerade in den Gebieten des Hügel- und Berglandes, in denen große, zusammenhängen- 


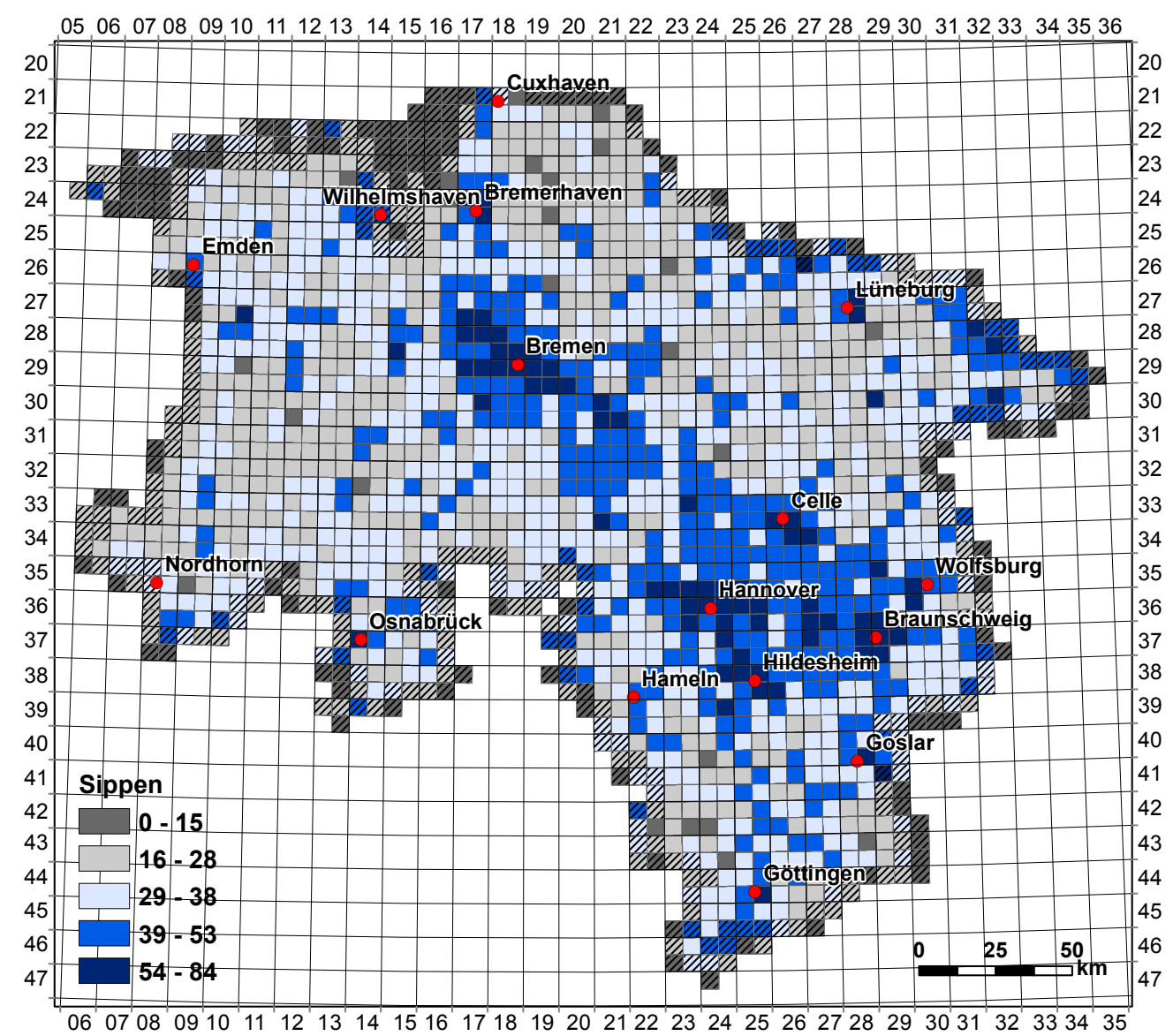

Abbildung 3.5: Sippenzahl je Quadrant der in Niedersachsen und Bremen vorkommenden 145 etablierten Neophyten.

Figure 3.5: Number of taxa per quadrant in Lower Saxony and Bremen, considering 145 established neophytes.

de Zentren der Sippendiversität der Indigenen und besonders geringe NeophytenDichten auftreten (Weserbergland, Göttinger Wald, Harz), sind jedoch auch noch besonders große, relativ zusammenhängende Waldflächen vorhanden. Natürliche und naturnahe Wälder, die in Mitteleuropa flächendeckend die potentiell natürliche Vegetationsformation darstellen (BoHN et al., 2000/2003), sind ein Refugium für eine Vielzahl der heimischen Arten. Dagegen ist die Anzahl der Neophyten in Wäldern geringer als im Offenland. Weniger als ein Drittel der in Niedersachsen etablierten Neophyten kommen nach ScHMIDT et al. (2003) regelmäßig in Wäldern vor. Ihre Schwerpunkte in bewaldeten Landschaften liegen auf Kahlschlagflächen und in bachund wegbegleitenden Vegetationstypen (vgl. CHYTRÝ et al., 2005).

Besonders hoch ist die Übereinstimmung der Verbreitung der Archäophyten mit der von etablierten Neophyten. Gemeinsam ist beiden Artengruppen, dass sie im 
Tabelle 3.5: Zahl der Gefäßpflanzensippen nach ihrem Einbürgerungsstatus (Indigene, Archäophyten und Neophyten) und ihrer jeweiligen Gefährdungssituation (ungefährdete und Rote-Liste-Arten) auf Grundlage der Gesamtflorenliste für Niedersachsen und Bremen.

Table 3.5: Vascular plant taxa richness sorted by naturalization status (indigenous, archaeophytes, established neophytes) and threat situation (non-threatened vs. Red List taxa) based on the complete floristic list of Lower Saxony and Bremen.

\begin{tabular}{lrrr}
\hline & Gesamtliste & Ungefährdete Sippen & Rote-Liste-Arten \\
\hline Indigene & 1509 & 925 & 584 \\
Archäophyten & 160 & 111 & 49 \\
Etablierte Neophyten & 145 & 136 & 9 \\
Sippen ohne floristischen Sta- & 5 & 4 & 1 \\
tus & & & \\
\hline
\end{tabular}

Gefolge des Menschen nach Mitteleuropa eingewandert sind. Die meisten Archäophyten sind in Acker-, Grünland- und Ruderalvegetation angesiedelt. Sie sind damit zum Teil wichtige Bestandteile von historisch bedeutsamen, heute gefährdeten Biotoptypen, die relativ kleinflächig in eine ansonsten intensiv genutzte Kulturlandschaft eingebettet sind. Dies zeigt sich auch an der Vielzahl der als gefährdet eingestuften Archäophyten. Der von KüHN et al. (2003) festgestellte hohe Anteil von Archäophyten in Lössgebieten stimmt in der vorliegenden Auswertung mit einem Schwerpunkt der Vorkommen dieser Artengruppe in den Lössbörden überein. Letztere sind seit langem besiedelte und heute intensiv genutzte Agrar- und Industrielandschaften, in denen sich auch Neophyten großflächig etablieren konnten.

Urbane Ballungsräume weisen in Niedersachsen und Bremen generell eine hohe Artenzahl auf. Dies stimmt mit den Ergebnissen anderer Untersuchungen, etwa denen von Haeupler (1974), Wania et al. (2006) und Lippe \& KowariK (2008) überein, die feststellten, dass urbane Lebensräume generell eine höhere Pflanzenartenzahl aufweisen als ländliche Gebiete gleicher Größe. Sie führten dies auf die hohe Landschaftsheterogenität in den vielfältig genutzten Räumen zurück, die in der Regel ein kleinräumiges Mosaik von standörtlich sehr heterogenen naturnahen und künstlich geschaffenen Lebensräumen darstellen und somit einer Vielzahl von Pflanzenarten als Wuchsorte dienen können. KüHN et al. (2003) stellten außerdem fest, dass die Flora deutscher Städte natürlicherweise artenreich ist. Sie begründeten dies damit, dass Städte oftmals in von vorneherein sehr artenreichen Landschaftsbereichen entstanden sind.

Besonders evident ist der Zusammenhang zwischen der Diversität und der Urbanität der untersuchten Raumeinheiten für die Neophyten. Zu ähnlichen Ergebnissen kamen auch andere Studien, die sich mit der Verbreitung dieser Artengruppe beschäftigen (PYšEK, 1998; Roy et al., 1999; Honnay et al., 2003; Chytrý et al., 2005; MASKelL et al., 2006; Bотнам et al., 2009; Nobis et al., 2009). Maskell et al. (2006) wiesen für den ländlichen Raum Großbritanniens nach, dass nichtheimische Arten sich vor allem 


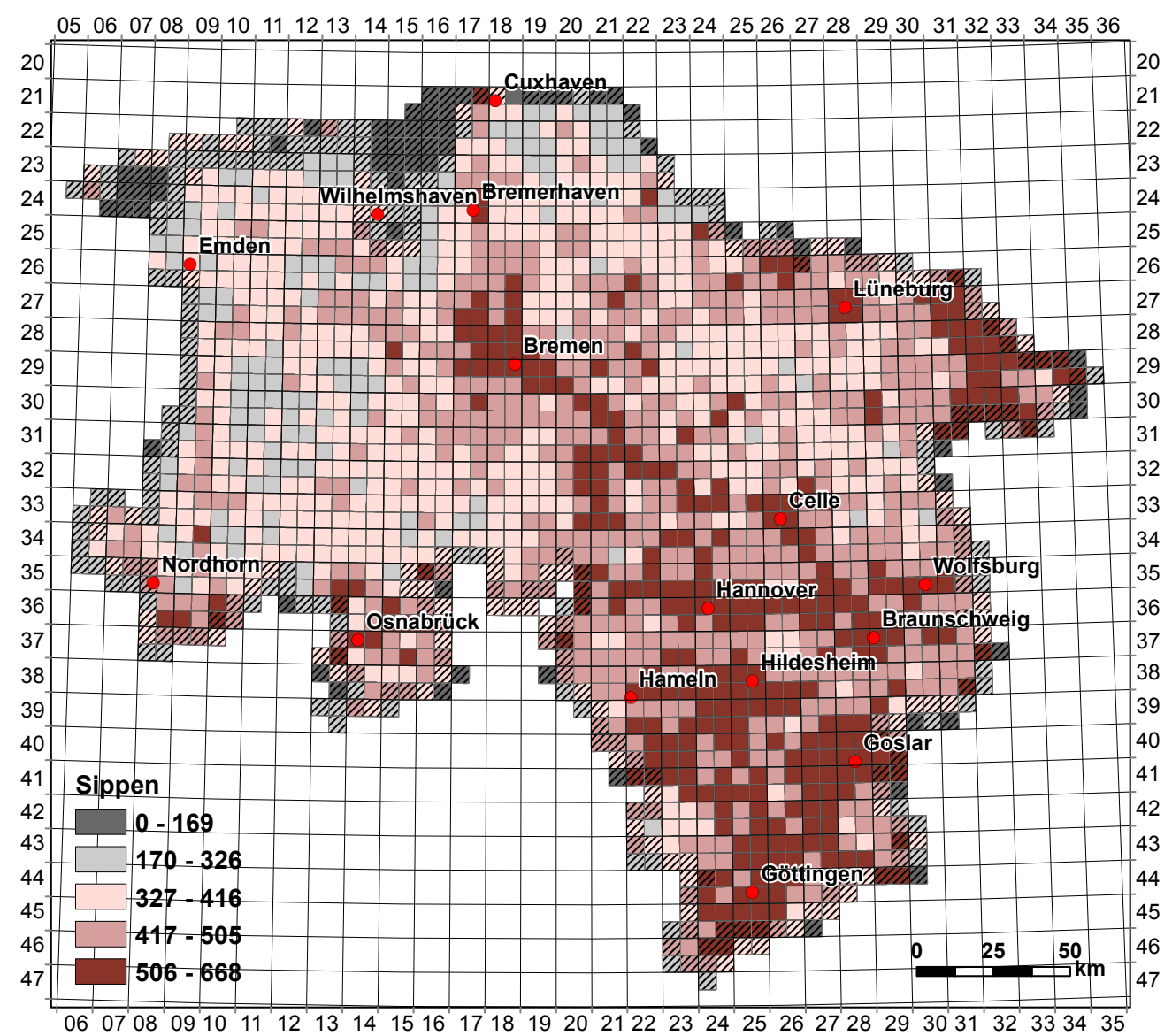

Abbildung 3.6: Anzahl der in Niedersachsen und Bremen je Quadrant vorkommenden 1176 ungefährdeten Gefäßpflanzensippen.

Figure 3.6: Number of taxa per quadrant in Lower Saxony and Bremen, considering 1176 invulnerable vascular plant taxa.

in vom Menschen geprägten Habitaten etablierten. Bотнам et al. (2009) untersuchten in Großbritannien innerhalb von zwei verschiedenen Untersuchungszeiträumen (1987-1988 und 2003-2004) die Bindung von indigenen Pflanzenarten, Archäophyten und Neophyten an verschiedene Landbedeckungsklassen. Sie stellten fest, dass Neophyten in beiden Zeitabschnitten stark an urbane Landschaftsbereiche gebunden waren, wohingegen die Archäophyten nur im ersten Untersuchungszeitraum stark mit urbanen Lebensräumen assoziiert waren. In geringerem Maße als für die Neophyten ist für die Archäophyten auch in unserer Studie ein Zusammenhang zwischen Urbanität und Artenreichtum zu erkennen. Diese Gruppe zeigt relativ kleinflächig ausgeprägte Diversitätszentren im Umfeld vieler niedersächsischer Städte. Aufgrund der Großräumigkeit der zugrundeliegenden Raumeinheiten konnte hier nicht untersucht werden, ob der Artenreichtum der Archäophyten, wie PYšEK (1998) bemerkt, 


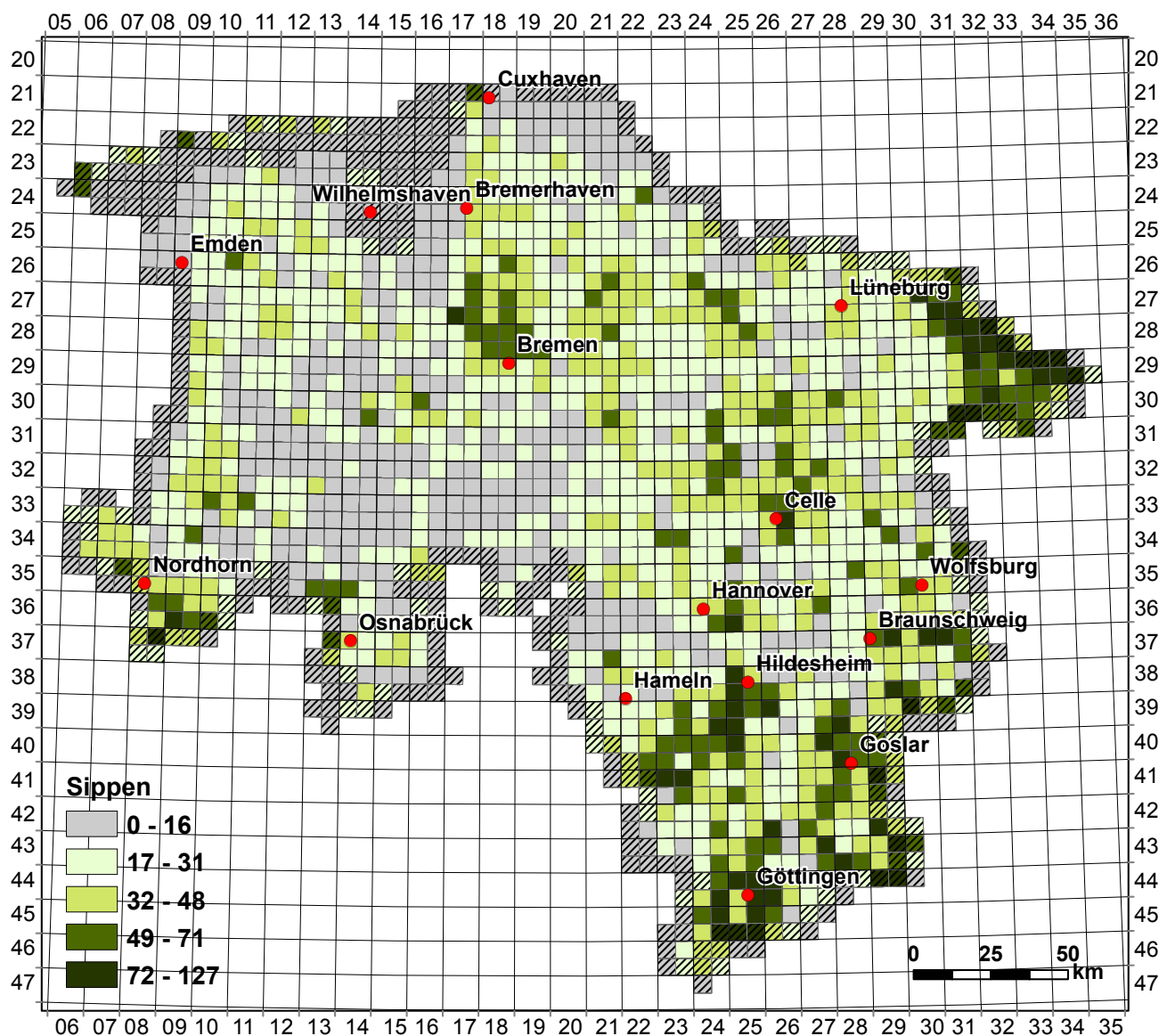

Abbildung 3.7: Anzahl der 643 seltenen und gefährdeten Gefäßpflanzensippen Niedersachsens und Bremens (Rote Liste-Status 1, 2, 3, G, R) je Quadrant.

Figure 3.7: Number of taxa per quadrant in Lower Saxony and Bremen, considering 643 rare and endangered vascular plant taxa (Red List status 1, 2, 3, G, R).

aufgrund ihrer Anpassung an ländliche Räume vor allem auch in kleineren Städten erhöht ist.

Haeupler \& Vogel (1999) sowie Haeupler (2000) betonen, dass die Musteranalyse von Rote-Liste-Artenzahlen gut geeignet ist, die 'echten' Hotspots der Gefäßpflanzendiversität aufzudecken. Für Niedersachsen und Bremen weisen die gefährdeten Arten ein wesentlich differenzierteres und eher punktuelles Verbreitungsmuster auf als die anderen betrachteten Artengruppen. Die Hotspots der Rote-Liste-Arten korrelieren in vielen Fällen mit denen der anderen Artengruppen, liegen jedoch nicht unmittelbar in den städtischen Ballungszentren. Bei den Rote-Liste-Arten handelt es sich fast ausschließlich um Indigene bzw. Archäophyten, wobei diese Arten oft Spezialisten sind, die an natürliche oder naturnahe Habitate angepasst sind. Eine besonders starke Überschneidung von Raumeinheiten mit hoher Dichte sowohl gefährdeter Arten 
als auch von Neophyten ergeben sich in Niedersachsen für das Wendland, die Umgebung von Göttingen, Hildesheim und Goslar sowie die Region nördlich von Bremen. Es kann jedoch davon ausgegangen werden, dass die Hauptursachen der Gefährdung von Pflanzenarten in der Intensivierung der Landnutzung und ihren Folgen (Eutrophierung, Entwässerung, Aufgabe von Extensivflächen) sowie dem gesteigerten Flächenbedarf der Bevölkerung liegen und nicht durch die Konkurrenz der Neophyten bedingt sind (GARvE, 2004).

\section{Danksagung}

Die Untersuchungen wurden im Rahmen des von der Deutschen Bundesstiftung Umwelt geförderten Projektes "Identifizierung von Indikatorartengruppen für ein Biodiversitäts-Monitoring zur Bewertung von Grünland- und Waldlebensräumen" (DBU 26752/33-0) durchgeführt. Besonderer Dank gilt den ehrenamtlichen Melderinnen und Meldern des Niedersächsischen Pflanzenarten-Erfassungsprogramms, ohne die wir das umfangreiche Wissen über die Verbreitung und Gefährdungssituation der Gefäßpflanzen in Niedersachsen und Bremen nicht hätten. Wir danken Prof. Dr. Henning Haeupler und einem anonymen Gutachter für wertvolle Anregungen zur Verbesserung einer früheren Version unseres Manuskripts. 


\section{Literaturverzeichnis}

Bohn, U.; Neuhäusl, R.; Gollub, G.; Hettwer, C.; Neuhäuslove, Z.; Schlüter, H. \& Weber, H. (2000/2003): Karte der natürlichen Vegetation Europas - Maßstab 1:2.500.000. Landwirtschaftsverlag, Münster.

Botham, M. S.; Rothery, P.; Hulme, P. E.; Hill, M. O.; Preston, C. D. \& Roy, D. B. (2009): Do urban areas act as foci for the spread of alien plant species? An assessment of temporal trends in the UK. Diversity and Distributions 15: 338-345.

Chytrú, M.; PYŠEK, P.; TichÝ, L.; Knollova, I. \& DAnihelKa, J. (2005): Invasions by alien plants in the Czech Republic: a quantitative assessment across habitats. Preslia 77: 339-354.

Deutschewitz, K.; Lausch, A.; KüHN, I. \& KLOtZ, S. (2003): Native and alien plant species richness in relation to spatial heterogeneity on a regional scale in Germany. Global Ecology and Biogeography 12: 299-311.

ESRI INC. (2007): ArcGis-ArcInfo 9.2.

GARVE, E. (1994): Atlas der gefährdeten Farn- und Blütenpflanzen in Niedersachsen und Bremen: Kartierung 1982 - 1992. Naturschutz und Landschaftspflege in Niedersachsen 30: 1-895.

GARve, E. (2004): Rote Liste und Florenliste der Farn- und Blütenpflanzen in Niedersachsen und Bremen. Informationsdienst Naturschutz Niedersachsen 24: 1-76.

GARVE, E. (2007): Verbreitungsatlas der Farn- und Blütenpflanzen in Niedersachsen und Bremen. Naturschutz und Landschaftspflege in Niedersachsen 43: 1-507.

Haeupler, H. (1974): Statistische Auswertung von Punktrasterkarten der Gefäßpflanzenflora Süd-Niedersachsens. Scripta Geobotanica 8: 141 S.

Haeupler, H. (2000): Die ungleiche Verteilung der Artenvielfalt innerhalb Deutschlands - Eine Antwort. Floristische Rundbriefe 34: 17-24.

Haeupler, H. \& Vogel, A. (1999): Plant diversity in Germany: a second review. Acta Botanica Fennica 162: 55-59.

Heineberg, H. (2006): Stadtgeographie. Schöningh, Paderborn. 3. Aufl., 481 S.

Heunisch, C.; Caspers, G.; Elbracht, J.; Langer, A.; Röhling, H.-G.; Schwarz, C. \& StreIf, H. (2007): Erdgeschichte von Niedersachsen: Geologie und Landschaftsentwicklung. GeoBerichte 6: 3-85.

Honnay, O.; Piessens, K.; Landuyt, W. v.; Hermy, M. \& Gulinck, H. (2003): Satellite based land use and landscape complexity indices as predictors for regional plant species diversity. Landscape and Urban Planning 63: 241-250. 
Hubo, C.; Jumpertz, E.; Krott, M.; Nockemann, N.; Steinmann, A. \& Bräuer, I. (2007): Grundlagen für die Entwicklung einer nationalen Strategie gegen invasive gebietsfremde Arten. BfN-Skripten 213: $370 \mathrm{~S}$.

Klotz, S.; KüHN, I. \& DuRKA, W. (2002): BIOLFLOR - Eine Datenbank zu biologischökologischen Merkmalen der Gefäßpflanzen in Deutschland. Schriftenreihe für Vegetationskunde 38: 1-334. URL http://www.biolflor.de.

KüHN, I.; BRANDL, R.; MaY, R. \& Klotz, S. (2003): Plant distribution patterns in Germany - Will aliens match natives? Feddes Repertorium 114: 559-573.

LIPPE, M. v. D. \& KowARIK, I. (2008): Do cities export biodiversity? Traffic as dispersal vector across urban-rural gradients. Diversity and Distributions 14: 18-25.

Maskell, L. C.; Firbank, L. G.; Thompson, K.; Bullock, J. M. \& Smart, S. M. (2006): Interactions between non-native plant species and the floristic composition of common habitats. Journal of Ecology 94: 1052-1060.

Niedersachsen (2008): Zahlen und Fakten. Niedersachsen im Vergleich: Ländervergleich Fläche. URL http:/ /www.niedersachsen.de. Abgerufen am 15.12.2010.

NLFB (Niedersächsisches Landesamt für Bodenforschung) (1997): Böden in Niedersachsen, Teil 1: Bodeneigenschaften, Bodennutzung und Bodenschutz. URL http://www.lbeg.de/extras/nlfbook/html/nds_main.htm. Abgerufen am 09.07.2010.

Nobis, M. P.; Jaeger, J. A. G. \& ZimmermanN, N. E. (2009): Neophyte species richness at the landscape scale under urban sprawl and climate warming. Diversity and Distributions 15: 928-939.

PYŠEK, P. (1998): Alien and native species in Central European urban floras: a quantitative comparison. Journal of Biogeography 25: 155-163.

PyšeK, P.; JarošíK, V. \& Kucera, T. (2002): Patterns of invasion in temperate nature reserves. Biological Conservation 104: 13-24.

R Development Core Team (2012): R: A language and environment for statistical computing. URL http://www.R-project.org.

Roy, D. B.; Hill, M. O. \& Rothery, P. (1999): Effects of urban land cover on the local species pool in Britain. Ecography 22: 507-515.

Schacherer, A. (2001): Das Niedersächsische Pflanzenarten-Erfassungsprogramm. Informationsdienst Naturschutz Niedersachsen 21 (5/01 Supplement Pflanzen): 1-20.

Schmidt, M.; Ewald, J.; Fischer, A.; Oheimb, G. v.; Kriebitzsch, W.-U. \& EllenberG, H. (2003): Liste der Waldgefäßpflanzen Deutschlands. Mitteilungen der Bundesforschungsanstalt für Forst- und Holzwirtschaft 212: 1-35. 
Schmitt, T. \& Haeupler, H. (2009): Hot Spots der Phytodiversität in Deutschland. Geographische Rundschau 61: 18-25.

Schnittler, M. \& Ludwig, L. (1996): Zur Methodik der Erstellung Roter Listen. Schriftenreihe für Vegetationskunde 28: 709-739.

SeKretariat Der CBD (2007): Globale Strategie zur Erhaltung der Pflanzen: Übersetzung der englischen Originalfassung von April 2002. 15 S.

Wagenitz, G. (2003): Wörterbuch der Botanik. Spektrum Akademischer Verlag, Heidelberg. 2. Aufl., $552 \mathrm{~S}$.

WaniA, A.; KüHN, I. \& KLOTZ, S. (2006): Plant richness patterns in agricultural and urban landscapes in Central Germany - spatial gradients of species richness. Landscape and Urban Planning 75: 97-110. 


\section{The effectiveness of protected areas for the conservation of threatened vascular plant species}

Schmiedel, I.; Schmidt, M.; Schacherer, A. \& Culmsee, H. (2013): Die Effektivität von Schutzgebieten für die Erhaltung seltener und gefährdeter Gefäßpplanzenarten. Eine Untersuchung im niedersächsischen Tiefland. Naturschutz und Landschaftsplanung 45: 45-52. 


\section{Summary}

The present study tests the effectiveness of protected areas for the conservation of rare and threatened vascular plant species. For this purpose, the lowland area of the federal state of Lower Saxony, Germany with its 41 geographical sub-regions served as a model region.

The analysis was conducted for nature protected areas ${ }^{1}$ and Natura 2000 sites that represent the most important and extensive conservation areas in the lowlands. Information on the occurrences of Red List (RL) species were obtained from the floristic mapping of the state (plant survey of Lower Saxony, NLWKN 1982-2003). In addition to the total number of RL species, those species linked to forest habitats ${ }^{2}$ (forest affinity categories 1.1 and 1.2, SснміDT et al., 2011) were considered in the analysis. All data were available on the basis of a regular grid of 15.729 grid cells with a size of $207 \pm 2$ ha. The study aimed at answering the following research questions: 1.) What patterns of diversity can be observed for the two species groups? 2.) Are occurrences of threatened species covered by nature protected areas? 3.) Does the designation of Natura 2000 sites improve the situation? 4.) Are the results obtained for RL species transferable to RL forest species?

The correlation between the extent of protected areas and the number of RL species per grid cell was analysed using Generalised Linear Models (GLM) with Poisson distribution. The calculation was performed separately for each of the 41 geographical sub-regions. The same kind of analysis was repeated for RL forest species.

Richness of the rare and threatened vascular plant species in the lowlands of Lower Saxony showed a distinct pattern. Centres of species richness (hotspots) were identified that should be considered as priority areas for nature conservation. Hotspots of RL species were mainly located in the Wendland area, the Westmünsterland and the Wesermünder Geest, those of RL forest species in the Oldenburger Geest. In contrast, large parts of the western lowlands as e. g. the Hunte-Leda-Moorniederung, the Bersenbrücker Land as well as the Bourtanger Moor were found to be very speciespoor. With the Ostmünsterland and the Rahden-Diepenauer Geest being an exception, hotspots of RL species were well covered by protected areas. In contrast, richness centres of RL forest species were effectively protected in only c. $60 \%$ of the geographical sub-regions. However, the number of species in those regions where no positive correlation between the distribution of species richness and the extent of protected areas could be found was generally very low. By including Natura 2000 sites the proportion of well-protected hotspots increased to an effective protection in $95 \%$ of the geographical sub-regions for RL species and $81 \%$ for RL forest species.

The diverging results obtained for the two species groups suggest that it is reasonable to not only focus on the total number of RL species representing a wide

\footnotetext{
${ }^{1}$ German: Naturschutzgebiete

${ }^{2}$ Below named 'RL forest species'.
} 
ecological amplitude as it has been done in most studies dealing with the effectiveness of protected areas for species conservation. Instead it is worthwhile to extent the analysis to species groups that represent more narrow habitat requirements. 


\section{Abstracts}

Die Einrichtung von Schutzgebieten ist ein zentrales Instrument des Artenschutzes. Am Beispiel der 41 naturräumlichen Regionen des niedersächsischen Tieflands wird ein Verfahren zur Überprüfung der Effektivität von Naturschutz- (NSG) und FFHGebieten (Fauna Flora Habitat) für den Schutz seltener und gefährdeter Gefäßpflanzenarten (Rote-Liste-Arten $=$ RL-Arten) vorgestellt. Grundlage sind die Daten des Niedersächsischen Pflanzenarten-Erfassungsprogramms.

In $85 \%$ der naturräumlichen Regionen ist die Gesamtzahl der RL-Arten durch NSG effektiv geschützt; für die an den Lebensraum Wald gebundenen RL-Arten hingegen trifft das nur in 61 \% der naturräumlichen Regionen zu. Die Ausweitung des Schutzgebietssystems durch FFH-Gebiete verbessert die Schutzsituation der RL-Arten insgesamt wie auch die der RL-Waldarten erheblich.

Der vorgestellte Ansatz ermöglicht die Identifizierung von Zentren der Artenvielfalt (Hotspots), die in Hinsicht auf ihren Schutz und ggf. gezielte konservierende Pflegemaßnahmen besondere Beachtung finden sollten.

\section{Effectiveness of protection areas for the conservation of rare and endangered vascular plant species - An investigation in the lowlands of Lower Saxony, Germany}

The establishment of protected areas is a central tool in species conservation. Using the 41 geographical regions of the Lowlands of Lower Saxony, Germany, as a model region, a method to test the effectiveness of nature protected areas and Natura 2000 sites for the conservation of rare and endangered vascular plant species (RL, Red List species) is proposed. RL species are effectively protected by nature protected areas in $85 \%$ of the geographical regions; for RL species linked to forest habitats this is the case only in $61 \%$ of the geographical regions. The extension of the protected area system to include Natura 2000 sites greatly improves the situation.

The approach allows the identification of regions with high species richness of endangered plants (hotspots) that should be particularly considered for conservation and management actions. 


\section{Einleitung}

Die Einrichtung von Schutzgebieten stellt ein zentrales Instrument von Strategien zur Verringerung von Biodiversitätsverlusten dar (MEA, 2005; GASTON et al., 2006; BMU, 2007). In diesem Zusammenhang fordert die 'Globale Strategie zur Erhaltung der Pflanzen' (GSPC, CBD-COP6, Entscheidung VI/9, SEKRETARIaT der CBD, 2007) einen effektiven in-situ-Schutz, durch den dem weiteren Rückgang von gefährdeten Pflanzenarten entgegengewirkt werden soll. Ob die für den Pflanzenartenschutz bedeutsamen Flächen auch tatsächlich durch Schutzgebiete abgedeckt sind, wurde bisher für größere Räume kaum untersucht. Für Deutschland fehlen Studien, die die Wirksamkeit der bestehenden Schutzgebietskulisse analysieren (ENGEL et al., 2012), und auch auf internationaler Ebene sind nur wenige Untersuchungen zur Effektivität von Schutzgebieten für den Artenschutz bekannt (DimitraKopoulos et al., 2004; Deguise \& KerR, 2006; JACKson et al., 2009).

Naturschutzgebiete (NSG) und Fauna-Flora-Habitat-Gebiete (FFH-Gebiete) des europäischen Netzwerks Natura 2000 sind in Deutschland die flächenmäßig bedeutsamsten Schutzgebietskategorien (BFN, 2008). Die Ausweisung von NSG kann aus wissenschaftlichen, naturgeschichtlichen und/oder landeskundlichen Gründen sowie wegen der Seltenheit, besonderen Eigenart oder hervorragenden Schönheit der Gebiete erfolgen ( $\$ 23$ BNatSchG, 2009). Der Artenschutz ist also nicht unbedingt explizites Kriterium für die Schutzgebietsausweisung. Im Rahmen der FFH-Richtlinie (Richtlinie 92/43/EWG) werden Schutzgebiete dagegen vorrangig nach Kriterien des Habitat- und Artenschutzes ausgewiesen (SsymANK, 1998). Hierbei wurden allerdings vielfach bestehende Schutzgebiete, insbesondere NSG, eingeschlossen. Für beide Schutzgebietskategorien gibt es keine allgemeingültigen Schutzbestimmungen - diese müssen durch Schutzgebietsverordnungen (NSG) bzw. Managementpläne (FFH-Gebiete) festgelegt werden.

Die vorliegende Studie untersucht die Effektivität von Schutzgebietssystemen (NSG und FFH-Gebiete) für die Erhaltung von seltenen und gefährdeten Gefäßpflanzen im niedersächsischen Tiefland. Dabei werden zunächst alle im niedersächsischen Tiefland vorkommenden Gefäßpflanzenarten der Roten Liste Niedersachsens betrachtet (490 Sippen; Rote Liste-Status der Tiefland-Region 1, 2, 3, G, R; GARVE, 2004; im Folgenden RL-Arten genannt).

Um feststellen zu können, ob die für die RL-Arten der Gesamtliste erhaltenen Ergebnisse gleichermaßen für einen Teil der an einen bestimmten Lebensraum gebundenen RL-Arten gelten, werden die Analysen beispielhaft für die seltenen und gefährdeten Waldarten (nach ScHMIDT et al., 2011; 60 Sippen; im Folgenden RL-Waldarten genannt) wiederholt, da die Datenlage zu dieser Artengruppe sehr gut ist und Waldlebensräume eine große ökologische Bedeutung besitzen (ML, 2004). Wälder, insbesondere solche mit langer Habitatkontinuität, nehmen im nordwestdeutschen Tiefland im Vergleich zum nordostdeutschen Tiefland und vielen deutschen Mittelgebirgsregionen einen sehr geringen Flächenanteil ein (GLASER \& HAUKE, 2004; SCHMIDT 
et al., 2009). Hinsichtlich der Nutzungsgeschichte und Größe der Wälder sowie ihres Fragmentierungsgrades bestehen zwischen den 41 naturräumlichen Regionen des Tieflands große Unterschiede, die sich sowohl in der Ausstattung mit seltenen und gefährdeten Waldgefäßpflanzenarten als auch in der Schutzgebietskulisse widerspiegeln sollten.

Die NSG und FFH-Gebiete im niedersächsischen Tiefland sind sehr unterschiedlichen Alters. Während die NSG teilweise bereits seit über 90 Jahren bestehen (PoHL, 1999), wurden die FFH-Gebiete als Teile des Natura-2000-Schutzgebietssystems erst seit 1997 an die EU gemeldet (NMUK, 2010).

Folgenden Fragestellungen wird nachgegangen:

1. Wie ist die Artenvielfalt der betrachteten Artengruppen im niedersächsischen Tiefland verteilt?

2. Inwieweit sind die Vorkommen der RL-Arten durch NSG abgedeckt?

3. Hat sich die Schutzgebietsabdeckung durch die Ausweisung von FFH-Gebieten verbessert?

4. Sind die für die RL-Arten der Gesamtliste erhaltenen Ergebnisse auf die RLWaldarten übertragbar?

\section{Methoden}

\section{Untersuchungsgebiet}

Die Untersuchungen beziehen sich auf das niedersächsische Tiefland (Abb. 4.1), das sich in fünf naturräumliche Großregionen mit 41 naturräumlichen Regionen (NR) gliedert (MeYnen \& Schmithüsen, 1953-1962, modifiziert nach PilgRim \& FRANKE, 1993). Das Untersuchungsgebiet (UG) ist durch geringe Reliefunterschiede geprägt und von pleistozänen Ablagerungen dominiert (Heunisch et al., 2007). Von West nach Ost besteht ein Kontinentalitätsgradient, der sich in mittleren Jahresniederschlags- sowie Temperaturgradienten ausdrückt und sich auch auf die Bodenzusammensetzung auswirkt (NLFB, 1997).

Die Ems-Hunte-Geest und Dümmer-Geestniederung im Südwesten des UG ist in ihrem nördlichen Teil von ausgedehnten, vielfach von Flugsanden oder Sandlöss bedeckten Grundmoränenplatten geprägt. Ihr Südteil wird von Talsandflächen, großflächigen Mooren und kleineren Grundmoränenplatten dominiert, die z. T. von Endmoränenzügen überragt werden. Der Waldanteil liegt bei $17 \%$, geschützte Waldbereiche nehmen $1 \%$ ein. Die Ostfriesisch-Oldenburgische Geest im Nordwesten liegt auf Grundmoränenplatten und wird intensiv landwirtschaftlich genutzt; Wälder haben einen Flächenanteil von 7\%. Das Weser-Aller-Flachland mit den Urstromtälern von Aller und Weser und den sich südlich anschließenden flachwelligen Moränenlandschaften liegt im Südosten des UG an der Grenze zum Hügel- und Bergland und hat mit $21 \%$ den zweithöchsten Waldanteil im Tiefland. Die im Norden gelegene 
Stader Geest ist durch Grundmoränen, Geesten sowie moorreiche Flussniederungen geprägt. Sie setzt sich aus kleinräumigen Acker-, Grünland-, Wald- und Moorgebieten zusammen. Wälder nehmen knapp $15 \%$ der Region ein. In Lüneburger Heide und Wendland im östlichen Teil des UG überwiegen sandige Grund- und Endmoränen. Die Region wird von Äckern, Wäldern und den größten Sandheiden Niedersachsens geprägt und hat mit 39\% den höchsten Waldanteil der Großregionen des UG.

\section{Gefäßpflanzendaten}

Die Verbreitungsdaten der im UG als selten oder gefährdet eingestuften Gefäßpflanzensippen (RL-Status der Tiefland-Region 1, 2, 3, G, R; GARVE, 2004) wurden der Datenbank des Niedersächsischen Pflanzenarten-Erfassungsprogramms entnommen (NLWKN 1982-2003, Stand 15.12.2008). Es gingen 490 Sippen (468 Arten, 14 Subspezies, 8 Aggregate) in die Analyse ein. Die Sippen wurden jeweils auf der höchsten in der Datenbank aufgeführten taxonomischen Ebene zusammengefasst. Die Analyse erfolgte auf Minutenfeld-Ebene (15 Minutenfelder pro Messtischblatt-Quadrant; insgesamt 15.729 Minutenfelder mit einer mittleren Größe von $207 \pm 2$ ha). Für die Teilanalyse der an den Wald gebundenen RL-Arten wurden aus der Gesamtliste diejenigen Sträucher und krautigen RL-Arten extrahiert, die im geschlossenen Wald sowie an Waldrändern und auf Waldverlichtungen vorkommen (Waldbindungskategorien 1.1, 1.2, SснмidT et al., 2011). Der Gruppe gehörten 60 Sippen (59 Arten, 1 Aggregat) an. Die Verbreitungsmuster der Arten wurden als Artenzahl pro Minutenfeld berechnet und in einem Geografischen Informationssystem (ESRI INC., 2007) visualisiert. Dabei wurden natürliche Grenzwerte mit jeweils fünf Klassen verwendet.

\section{Schutzgebietssysteme}

Die Ausdehnung und Lage der NSG und FFH-Gebiete wurden digitalen Daten entnommen (NLWKN, 2010a; NLWKN, 2010b, Stand: 31.12.2009). Für die Teilanalyse der an den Wald gebundenen RL-Arten wurden die digitalen Schutzgebietsdaten mit dem ATKIS-DLM-Layer Wald (LGN, Hannover) verschnitten. In die Analyse wurden zum einen durch NSG, zum anderen zusätzlich auch die durch FFH-Gebiete geschützten (Wald-)Flächen einbezogen und ihr Flächenanteil je Minutenfeld berechnet.

\section{Effektivitäts-Analyse}

Der Zusammenhang zwischen der Ausdehnung der Schutzgebiete (NSG bzw. NSG und FFH-Gebiete) bzw. der geschützten Waldflächen (durch NSG bzw. NSG und FFH-Gebiete geschützte Waldflächen) und der Anzahl von RL-Arten bzw. RLWaldarten pro Minutenfeld wurde mit Generalisierten Linearen Modellen mit Poisson-Verteilung untersucht. Es wurden einzelne Analysen für jede der 41 naturräumlichen Regionen berechnet. Die Schutzgebietsfläche je Minutenfeld war dabei 
die erklärende, die Artenzahl die abhängige Variable. Die Datenanalyse erfolgte mit dem Statistikprogramm R (R Development Core Team, 2012).

\section{Habitatbindung der RL-Waldarten}

Für die weitere Interpretation wurde die Habitatbindung der RL-Waldarten gemäß FloraWeb-Datenbank (BFN, 2010; Haupt- und Schwerpunktvorkommen) und GARVE (1994) ermittelt (bis zu drei Mehrfachnennungen je Art).

\section{Ergebnisse}

\section{Verbreitung der RL-Arten}

Im UG wurden insgesamt 490 RL-Arten nachgewiesen. Die absolute Artenzahl variierte zwischen den Großregionen stark. Während sie maximal bei 454 RLArten lag (Lüneburger Heide und Wendland), kamen im Minimum in der Ostfriesisch-Oldenburgischen Geest nur 255 Arten vor (Tab. 4.1). Die räumliche Verteilung von RLHotspots war deutlich inhomogen (Abb. 4.2). Maximal kamen pro Minutenfeld 86 Arten, im Mittel $5( \pm 6)$ Arten vor. Weniger als $1 \%$ der Minutenfelder wies mehr als 28 Arten auf, wobei die größte Dichte dieser Hotspots im Wendland lag (NR 860, 876). Rund 7\% der Minutenfelder waren sehr artenreich (15-28 Arten). Das westliche Tiefland war allgemein artenarm mit punktuell großflächigen Hotspots im Bentheimer Raum (NR 544, 580), im Emsgebiet (NR 586, 592, 604) und nordwestlich von Oldenburg (NR 602, 603). Im östlichen Tiefland war die Artendichte pro Minutenfeld tendenziell höher. Großflächige Hotspots lagen vor allem im Elbe-Weser-Dreieck (NR 631-633), in der Allerniederung (NR 626, 627), der Lüneburger Heide (NR 641, 642) und im Wendland. Die Teilmenge der an Waldhabitate gebundenen RL-Arten lag mit 60 Arten bei $12 \%$. Die Verbreitungsmuster der RL-Waldarten (Abb. 4.3) unterschieden sich stark von denen der Gesamtliste. Maximal kamen 22 Arten, im Mittel nur 0,5 ( \pm 1,3) Arten pro Minutenfeld vor (Tab. 4.1). In 76\% der Minutenfelder konnte keine Art nachgewiesen werden. Die Hotspots mit mehr als sieben Arten waren nur punktuell ausgebildet und umfassten weniger als 1\% der Minutenfelder. Räume mit höheren Artendichten waren die Oldenburger Geest (NR 603), das Elbe-Weser-Dreieck (NR 633, 634), das Uelzener und Bevenser Becken (NR 643) sowie die Burgdorf-Peiner Geestplatten und das Ostbraunschweigische Flachland (NR 623, 624). Das Wendland wie auch die im äußersten Westen des UG gelegenen Gebiete, die bei Betrachtung der Gesamtliste die höchsten RL-Artenzahlen aufwiesen, waren vergleichsweise arm an RL-Waldarten. 


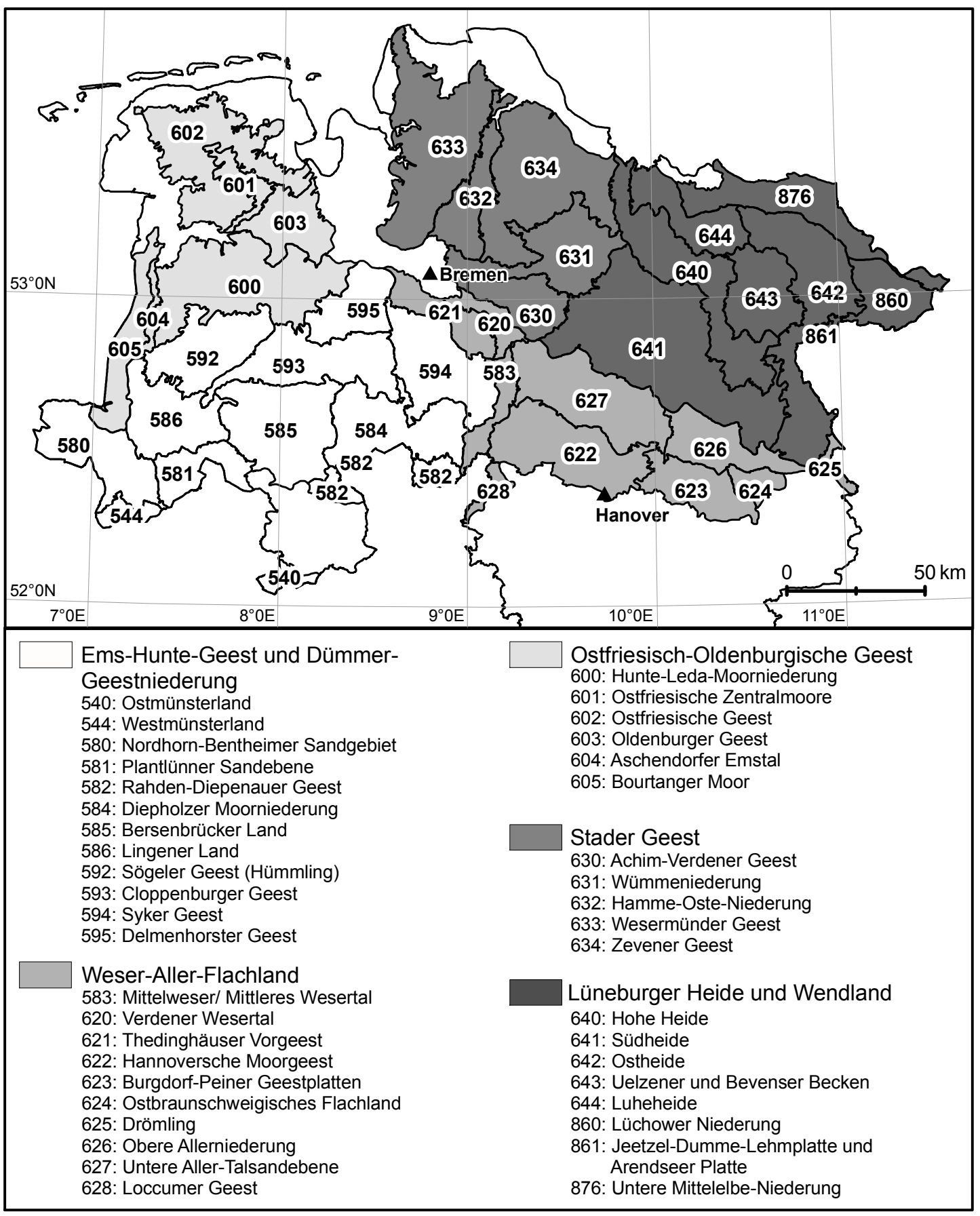

Abbildung 4.1: Das Untersuchungsgebiet des niedersächsischen Tieflandes mit seinen fünf naturräumlichen Großregionen und 41 naturräumlichen Haupteinheiten (MEYNEN \& ScHMITHÜSEN, 1953-1962, modifiziert nach PILGRIM \& FRANKe, 1993). Bezugssystem: Deutsches Hauptdreiecksnetz (DHDN, Gauss-Krüger, Zone 3). 


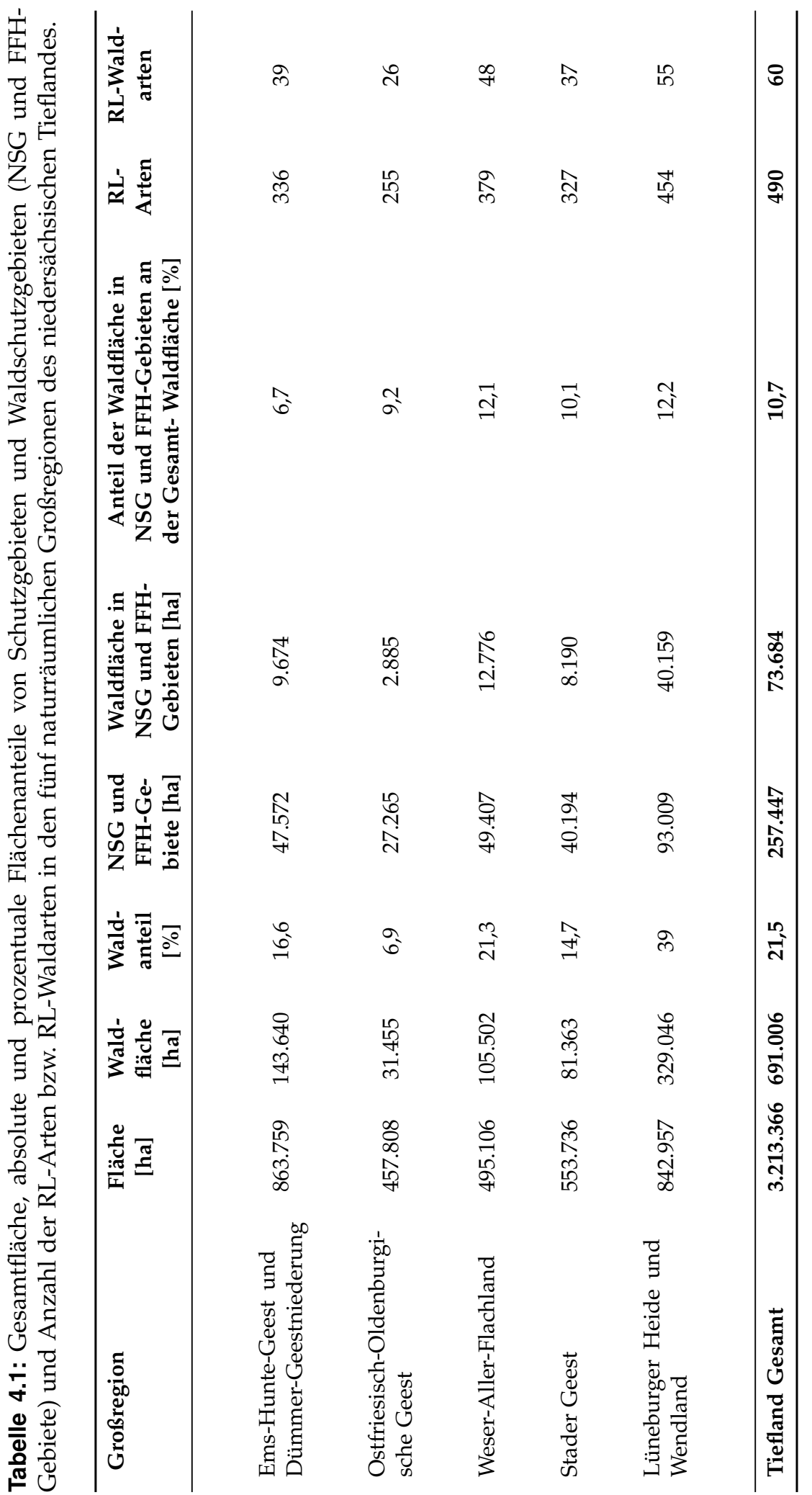




\section{Schutzgebietssysteme}

Im UG waren 530 NSG ausgewiesen, die insgesamt 140.439 ha $(4,4 \%)$ der Gesamtfläche einnahmen. Die 240 FFH-Gebiete erfassten mit 212.962 ha 6,6\% der Tieflandfläche. Durch eine starke Überlappung der Schutzgebietskategorien (Abb. 4.4) summierte sich die insgesamt von NSG und FFH-Gebieten abgedeckte Fläche im UG auf 257.445 ha (8\% der Gesamtfläche; Tab. 4.1). Durch NSG geschützte Wälder nahmen 41446 ha (1,3\% der Gesamtfläche), durch FFH-Gebiete geschützte Wälder 64.791 ha (2\%) ein. Der Anteil der insgesamt durch NSG und FFH-Gebiete geschützten Waldbereiche lag bei 2,3\%, der Anteil an der Waldfläche bei 10,7\% (Tab. 4.1).

\section{Effektivität von Schutzgebieten für den Artenschutz}

Die Vorkommen und Verbreitungsschwerpunkte der RL-Arten lagen zum überwiegenden Teil in Schutzgebieten (Tab. 4.2a). Die Effektivitätsanalyse zeigte für $85 \%$ der NR einen positiven Zusammenhang zwischen NSG- und RL-Arten-Vorkommen, in den übrigen NR bestand kein Zusammenhang. In der Unteren Mittelelbe-Niederung (NR 876), die eine außergewöhnlich hohe RL-Artenzahl und -dichte aufwies, lagen die Artvorkommen überwiegend außerhalb von NSG. Mit der Erweiterung der Schutzgebietskulisse um FFH-Gebiete (ca. 117.000 ha) erhöhte sich die Anzahl der NR mit Gebietsschutz auf 95\%. Dabei nahm z.B. die Schutzgebietsfläche in der Loccumer Geest von $<1 \%$ auf $18 \%$ zu (Abb. $4.5 \mathrm{a} / \mathrm{b}$ ), im RL-Hotspot Untere Mittelelbe-Niederung war sogar ein Anstieg von $<1 \%$ auf $24 \%$ zu verzeichnen. Lediglich in zwei naturräumlichen Regionen, in denen keine nennenswerte Veränderung der Schutzgebietsfläche stattfand, wurde auch weiterhin keine effektive Abdeckung der Artvorkommen durch Schutzgebiete erreicht.

Die Hotspots der RL-Waldarten waren deutlich schlechter durch Schutzgebiete abgedeckt als die der RL-Arten der Gesamtliste (Tab. 4.2b). Lediglich in $61 \%$ der NR lagen die Artvorkommen überwiegend in als NSG geschützten Wäldern. Durch die Ausweisung der FFH-Gebiete stieg der Anteil der NR mit einer guten Schutzgebietsabdeckung der Vorkommen der RL-Waldarten jedoch auf insgesamt $81 \%$. Markante Beispiele für diese Entwicklung sind die Oldenburger Geest (NR 603) und die Burgdorf-Peiner Geestplatten (NR 623). Hier sind die Minutenfelder mit besonders hohen Artendichten ( $>11$ RL-Waldarten) nicht durch NSG geschützt, wurden jedoch in die FFH-Gebietskulisse einbezogen (Tab. 4.2b, Abb. 4.5c/d).

Gegenüber der Schutzgebietsabdeckung der Vorkommen der RL-Arten insgesamt waren die Vorkommen der RL-Waldarten damit zwar deutlich weniger stark durch Schutzgebiete gesichert, allerdings waren die verbleibenden NR, in denen kein Zusammenhang von RL-Waldartenverteilung und Schutzgebieten erkennbar war, generell relativ artenarm. Eine Ausnahme bildete lediglich die Rahden-Diepenauer Geest (NR 582), in der sowohl die RL-Arten insgesamt als auch die mit einer vergleichsweise hohen Zahl vorkommenden RL-Waldarten auch nach der Ausweisung von FFH-Gebieten nicht ausreichend durch Schutzgebiete gesichert waren. 


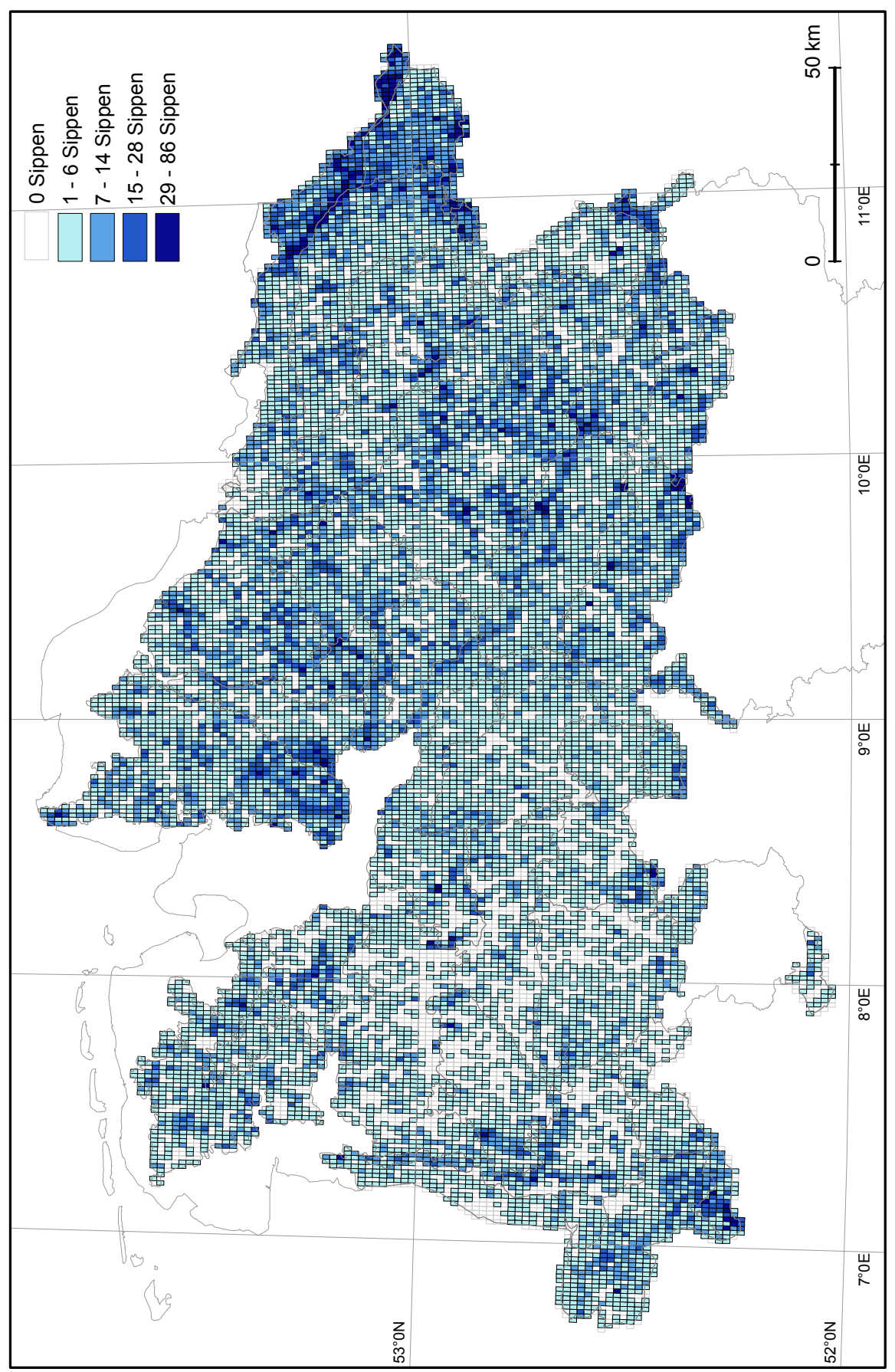

Abbildung 4.2: Anzahl der seltenen und gefährdeten Gefäßpflanzensippen pro Minutenfeld im niedersächsischen Tiefland. Insgesamt gingen 490 Sippen in die Berechnung ein. 


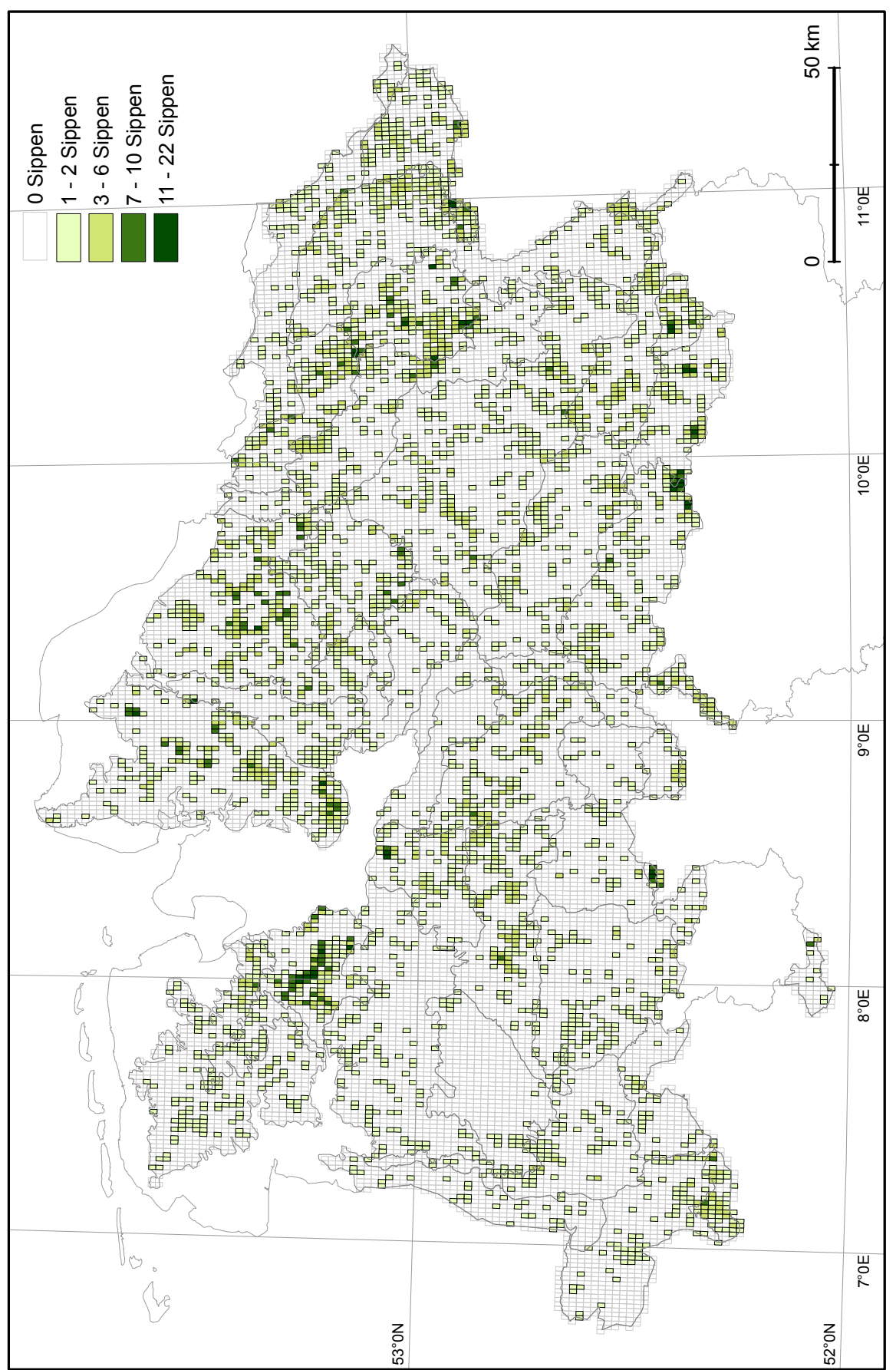

Abbildung 4.3: Anzahl der seltenen und gefährdeten Waldgefäßpflanzensippen pro Minutenfeld im niedersächsischen Tiefland. Insgesamt gingen 60 Sippen in die Berechnung ein. 


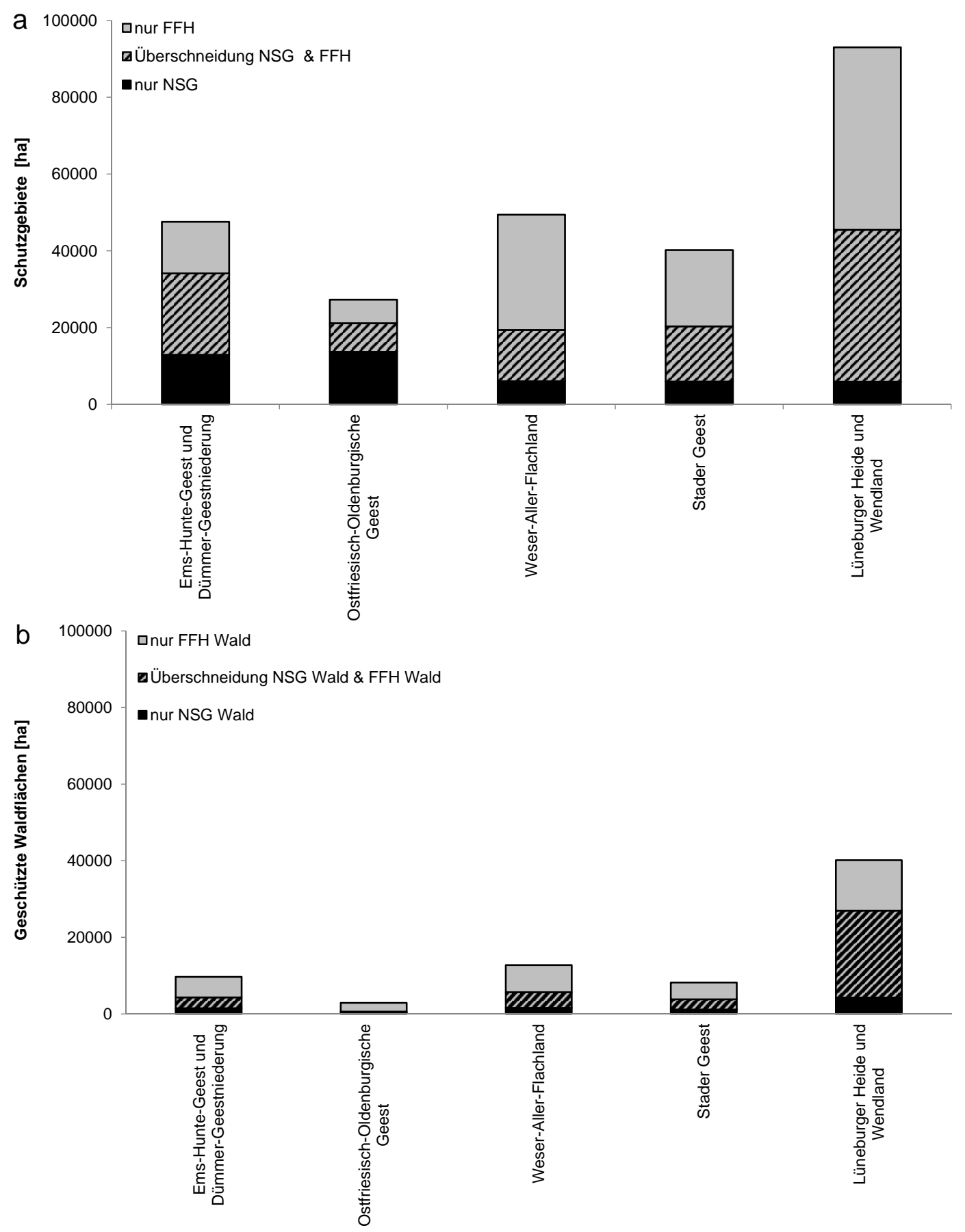

Abbildung 4.4: Schutzgebietsflächen (in Hektar) in den fünf Großregionen des niedersächsischen Tieflands unter Berücksichtigung der räumlichen Überlagerung von NSG und FFHGebieten. a) Gesamte Schutzgebietsfläche, b) Schutzgebiete in Wäldern. 


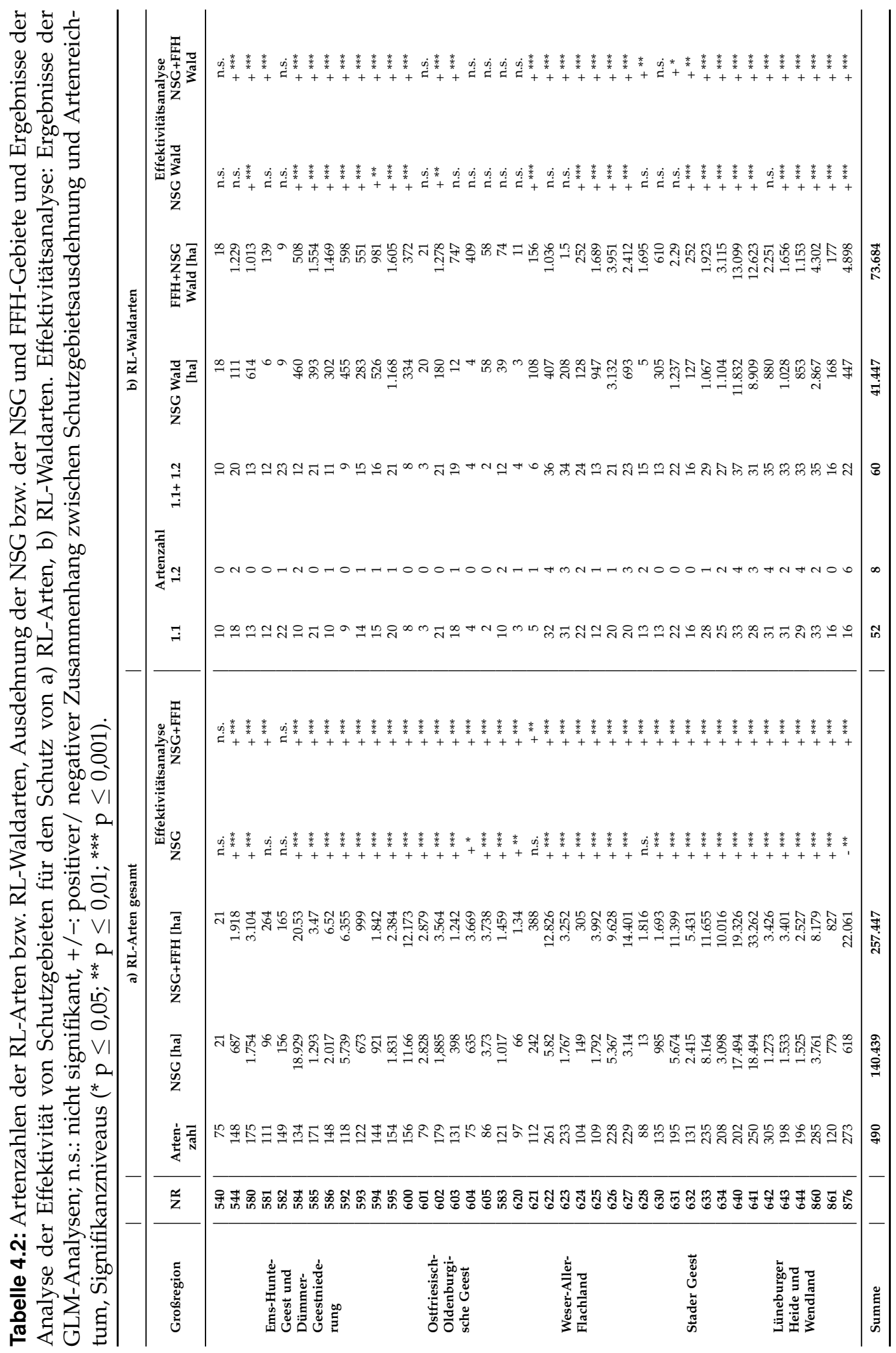



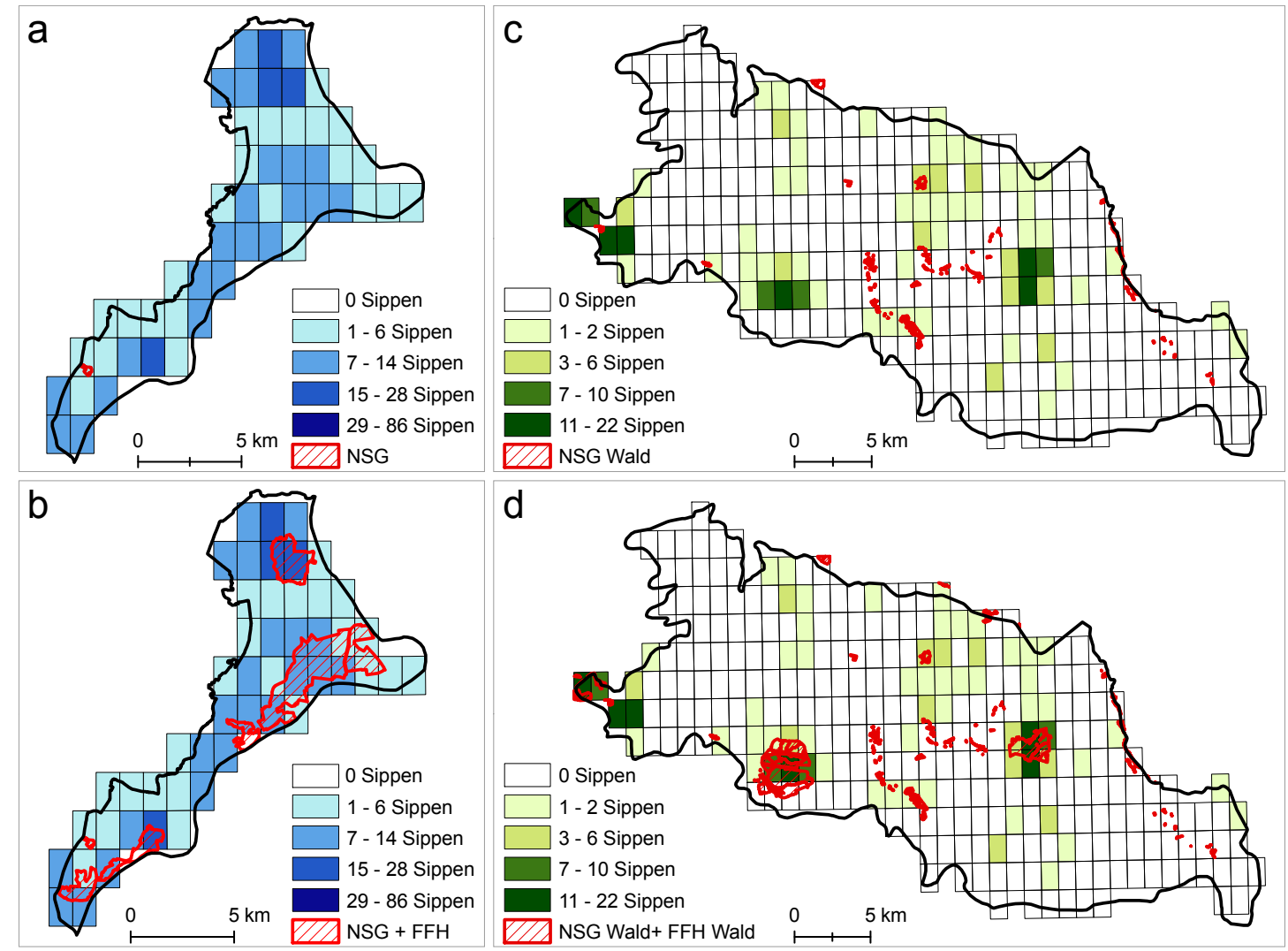

Abbildung 4.5: Beispiele für die Korrelation zwischen Schutzgebietsausdehnung und den Vorkommen der RL-Arten bzw. RL-Waldarten je Minutenfeld. In der Loccumer Geest (NR 628) überlagern sich die durch NSG geschützten Flächen und die Vorkommen der RL-Arten nicht (a), während sich unter Einbeziehung der FFH-Gebiete eine positive Korrelation zwischen Schutzgebietsausdehnung und den Vorkommen der RL-Arten zeigt (b). In den BurgdorfPeiner-Geestplatten (NR 623) sind die Vorkommen der RL-Waldarten nicht durch NSG abgedeckt (c), während sich unter Einbeziehung der FFH-Gebiete eine positive Korrelation zwischen Schutzgebietsausdehnung und den Vorkommen der RL-Waldarten zeigt (d).

\section{Diskussion}

Die Verbreitung der seltenen und gefährdeten Gefäßpflanzenarten im niedersächsischen Tiefland zeigt ein differenziertes Bild (vgl. auch ScHMIEDEL et al., 2011). Es lassen sich Zentren der Artenvielfalt identifizieren, die im Sinne einer "HotspotsStrategie" (Meyer et al., 2009) aus der landesweiten Perspektive als NaturschutzVorranggebiete angesehen werden müssen. Solche Hotspots finden sich vor allem im Wendland, im Westmünsterland und der Wesermünder Geest für RL-Arten bzw. der Oldenburger Geest für RL-Waldarten. Dagegen ist ein Großteil des westlichen Tieflands, wie etwa die Hunte-Leda-Moorniederung, das Bersenbrücker Land und das Bourtanger Moor, sehr artenarm. 
Die Vorkommen der RL-Arten insgesamt sind nur in wenigen Landschaftsräumen des UG nicht durch Schutzgebiete gesichert (z. B. Ostmünsterland, Rahden-Diepenauer Geest). Die Vorkommen der RL-Waldarten sind zwar in einer geringeren Zahl der naturräumlichen Regionen effektiv durch NSG und FFH-Gebiete geschützt, allerdings ist die Zahl der RL-Waldarten in den Naturräumen mit nur geringfügiger Schutzgebietsabdeckung auch sehr klein. Gründe hierfür sind in diesen Regionen im geringen Anteil alter Waldstandorte und im insgesamt niedrigen Waldanteil zu suchen.

Im Allgemeinen verbessert sich die Schutzsituation für beide betrachteten Artengruppen bei Berücksichtigung von FFH-Gebieten. Dies zeigt, dass die erst in jüngerer Zeit ausgewiesenen FFH-Gebiete im niedersächsischen Tiefland künftig neben den NSG bei entsprechender Maßnahmenplanung eine wichtige Rolle für den Schutz seltener und gefährdeter Gefäßpflanzenarten spielen könnten. In diesem Zusammenhang sollte geprüft werden, ob der durch die Untersuchung bestätigte effektive Gebietsschutz auch in allen Fällen einen effektiven Artenschutz impliziert (vgl. RoDRIGUES et al., 2004). Die Effektivität der Schutzgebiete für den Artenschutz ist in hohem Maße von den in den Schutzgebietsverordnungen bzw. Pflege- oder Managementplänen der Gebiete festgelegten Handlungsanweisungen abhängig. Insbesondere bei älteren NSG sind diese z. T. sehr unbestimmt formuliert (vgl. ENGEL et al., 2012) und für FFH-Gebiete derzeit noch in Arbeit. In vielen Fällen ist der Artenschutz zudem nicht vorrangiges Ziel bei der Schutzgebietsausweisung. Zukünftig sollten daher bei der Erarbeitung und Fortschreibung von Pflege- und Managementplänen in Schutzgebieten mit Hotspots zur dauerhaften Sicherung der bestehenden Populationen gezielt die Belange des Artenschutzes berücksichtigt werden.

$\mathrm{Zu}$ den Faktoren, die die Effektivität der Schutzgebiete einschränken können, zählen vor allem eine zu geringe Flächengröße der Gebiete bzw. ein hoher Grad an Isolierung: Sind die Schutzgebiete zu klein bzw. sind sie zu stark isoliert, können möglicherweise überlebensfähigen Populationen der schützenswerten Arten nicht erhalten werden (Deguise \& KeRR, 2006; JACKsON et al., 2009). Da ein Schutz größerer Flächen jedoch vielfach nicht umsetzbar ist, erscheint es in diesem Zusammenhang umso wichtiger, nicht allein auf die Wirkung von Schutzgebieten zu setzen, sondern im Rahmen einer "dualen Naturschutzstrategie" (JACKsON et al., 2009) auch Landschaftsbereiche außerhalb von Schutzgebieten so zu bewirtschaften, dass sie Populationen seltener und gefährdeter Arten nachhaltig als Lebensraum dienen können (DEGUISE \& KerR, 2006; OtтE et al., 2008). In diesem Zusammenhang können beispielsweise die durch Eigenbindung ausgewiesenen Waldschutzgebiete im niedersächsischen Landeswald eine wichtige Funktion erfüllen (NiEdERsächSISCHE LANDESFORSTEN, 2011).

Die für die Artengruppen voneinander abweichenden Ergebnisse zeigen, dass es sinnvoll ist, den Fokus nicht, wie in den bisher vorliegenden Studien zur Effektivität von Schutzgebieten (z. B. Deguise \& KerR, 2006; JACKson et al., 2009; VellaK et al., 2009), lediglich auf die Gesamtheit der seltenen und gefährdeten, eine große 


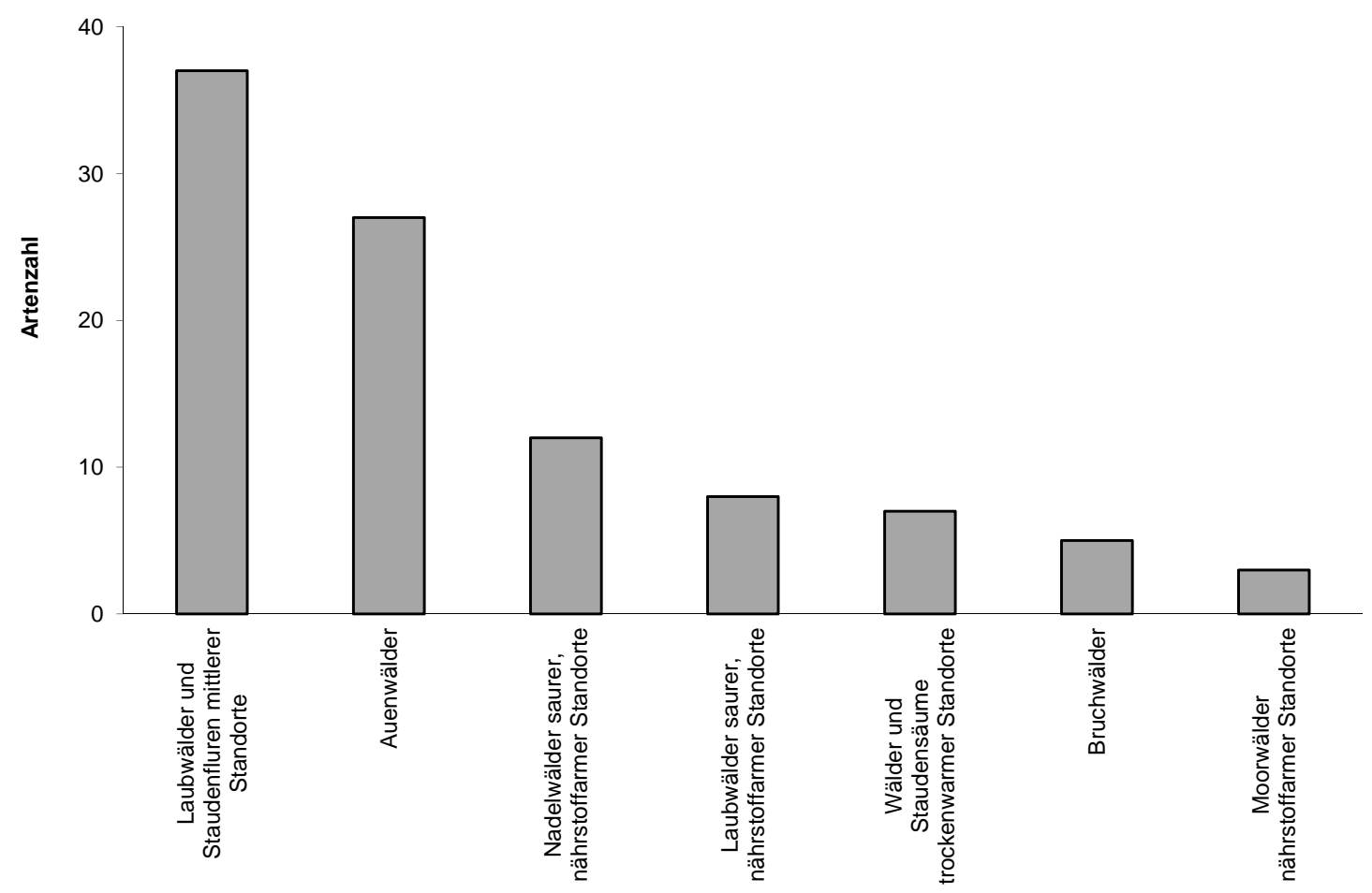

Abbildung 4.6: Habitatbindung der seltenen und gefährdeten Waldgefäßpflanzenarten des Tieflandes (bis zu drei Mehrfachzuordnungen pro Art).

standörtliche Amplitude repräsentierenden Gefäßpflanzenarten zu legen. Stattdessen kann die Analyse stärker differenziert und vergleichend auch solche Artengruppen einbezogen werden, die auf einen bestimmten Lebensraumtyp spezialisiert sind und somit eine geringere Standortamplitude aufweisen. Im vorliegenden Beispiel wurden hierfür die auf Wälder spezialisierten RL-Arten ausgewählt, da gerade Wäldern als der großflächig potenziell natürlichen Vegetation in Deutschland im Hinblick auf den Arten- wie auch den Prozessschutz eine große Bedeutung zukommt (BMU, 2007). Im niedersächsischen Tiefland zeigt ein Großteil der RL-Waldarten eine starke Habitatbindung an Laubwälder mittlerer Standorte (Waldmeister-Buchenwälder, StieleichenHainbuchenwälder) und Auenwälder (Abb. 4.6). Von den 60 RL-Waldarten waren 43 an einen dieser beiden Habitattypen gebunden.

Die RL-Gefäßpflanzenarten können, obwohl sie nur einen Baustein des Gesamtökosystems darstellen, aufgrund ihrer Sensibilität gegenüber äußeren Einflüssen als gute Indikatoren für intakte Ökosysteme und die an diese gebundenen Arten und Lebensgemeinschaften angesehen werden.

Die fortlaufend durch das Pflanzenarten-Erfassungsprogramm erhobenen Verbreitungsdaten der RL-Arten sind eine sehr gute Grundlage für ein Monitoring der Effektivität der Schutzgebiete im UG. Wiederholende Untersuchungen in den folgen- 
den Jahren werden zeigen, ob der in der vorliegenden Untersuchung belegte effektive Gebietsschutz langfristig auch zu einem effektiven Artenschutz führt.

\section{Fazit für die Praxis}

- Im niedersächsischen Tiefland liegen sowohl die Hotspots der Rote-Liste-Arten (RL-Arten) insgesamt als auch die der an Waldlebensräume gebundenen RLArten überwiegend in Schutzgebieten (NSG, FFH-Gebiete); die Ausweisung von FFH-Gebieten verbessert den Gebietsschutz für die RL-Arten in den meisten naturräumlichen Regionen erheblich. Wiederholungen der Untersuchungen werden zeigen müssen, ob der effektive Gebietsschutz auch zu einem effektiven Artenschutz führt.

- Bei der Erarbeitung und Fortschreibung von Pflege- und Managementplänen in Schutzgebieten mit Hotspots sollten künftig gezielt die Belange des Artenschutzes berücksichtigt werden, um die bestehenden Populationen von RL-Arten dauerhaft zu sichern.

- Die aufgezeigten Methoden der Hotspot-Analyse sollten stärker im Sinne einer "dualen Naturschutzstrategie" genutzt werden, um wichtige Populationen von RL-Arten auch außerhalb von Schutzgebieten durch nachhaltige Bewirtschaftungsformen zu erhalten.

- Rasterbasierte Verbreitungsdaten von RL-Arten sollten in regelmäßigen Zeitabständen aktualisiert werden, um sie als Grundlage eines Monitorings der Effektivität von Schutzgebieten für den Artenschutz einsetzen zu können.

\section{Dank}

Die Untersuchungen wurden dankenswerterweise durch die Deutsche Bundesstiftung Umwelt gefördert (DBU Az. 26752). 


\section{Literaturverzeichnis}

BfN (Bundesamt FÜR NATURSChutz) (2008): Daten zur Natur. Landwirtschaftsverlag, Münster. 368 S.

BfN (Bundesamt FÜr NATURschutz) (2010): FloraWeb: Daten und Informationen zu Wildpflanzen und zur Vegetation Deutschlands. URL http://www.floraweb.de. Abgerufen am 15.09.2010.

BMU (Bundesministerium FÜr UMWeLt, NATURSCHUtZ UND REAKTORSICHERHEIT) (2007): Nationale Strategie zur biologischen Vielfalt. 178 S.

Deguise, I. E. \& Kerr, J. T. (2006): Protected areas and prospects for endangered species conservation in Canada. Conservation Biology 20: 48-55.

Dimitrakopoulos, P. G.; Memtsas, D. \& Troumbis, A. Y. (2004): Questioning the effectiveness of the Natura 2000 Special Areas of Conservation strategy: the case of Crete. Global Ecology and Biogeography 13: 199-207.

Engel, F.; Wildmann, S. \& Meyer, P. (2012): Biodiversität im Wald braucht Planung: Was schützen wir und wie? Loccumer Protokolle 54: 119-136.

ESRI INC. (2007): ArcGis-ArcInfo 9.2.

GARVE, E. (1994): Atlas der gefährdeten Farn- und Blütenpflanzen in Niedersachsen und Bremen: Kartierung 1982 - 1992. Naturschutz und Landschaftspflege in Niedersachsen 30: 1-895.

GARVE, E. (2004): Rote Liste und Florenliste der Farn- und Blütenpflanzen in Niedersachsen und Bremen. Informationsdienst Naturschutz Niedersachsen 24: 1-76.

Gaston, K. J.; Charman, K.; Jackson, S. F.; Armsworth, P. R.; Bonn, A.; Briers, R. A.; Callaghan, C. S. Q.; Catchrole, R.; Hopkins, J.; Kunin, W. E.; Latham, J.; Opdam, P.; Stoneman R.; Stroud, D. A. \& Tratt, R. (2006): The ecological effectiveness of protected areas: The United Kingdom. Biological Conservation 132: 76-87.

Glaser, F. F. \& Hauke, U. (2004): Historisch alte Waldstandorte und Hudewälder in Deutschland: Ergebnisse bundesweiter Auswertungen. Angewandte Landschaftsökologie 61: 1-193.

Heunisch, C.; Caspers, G.; Elbracht, J.; Langer, A.; Röhling, H.-G.; Schwarz, C. \& STREIF, H. (2007): Erdgeschichte von Niedersachsen: Geologie und Landschaftsentwicklung. GeoBerichte 6: 3-85. 
JACKSON, S. F.; WALKER, K. \& GASTON, K. J. (2009): Relationship between distributions of threatened plants and protected areas in Britain. Biological Conservation 142: 1515-1522.

MEA (2005): Ecosystems and human well-being: Synthesis. Island Press, Washington. $137 \mathrm{~S}$.

Meyer, P.; Schmidt, M. \& Spellmann, H. (2009): Die Hotspots-Strategie - WaldNaturschutzkonzept auf landschaftsökologischer Grundlage. AFZ/ Der Wald 64: 822-824.

Meynen, E. \& Schmithüsen, J. (1953-1962): Handbuch der naturräumlichen Gliederung Deutschlands. Selbstverlag, Remagen. 1339 S.

ML (Niedersä̈HSISCHES Ministerium fÜR DEN LÄNDLICHEN RAUM ERNÄHRUNG LANDWIRTSChaft UND VerbRAUCherschutz) (2004): Der Wald in Niedersachsen: Ergebnisse der Bundeswaldinventur II. Aus dem Walde - Schriftenreihe Waldentwicklung in Niedersachsen 55: 1-43.

NiedersäChSIsche LANDESforsten (2011): Das LÖWE-Programm: 20 Jahre langfristige ökologische Waldentwicklung. URL http://www.landesforsten.de/ LOEWE-Langfristige-OEkologische-Waldentwicklung.20.0.html. Abgerufen am 26.07.2012.

NLFB (NiedersäChSISCHES LANDESAMt FÜR Bodenforschung) (1997): Böden in Niedersachsen, Teil 1: Bodeneigenschaften, Bodennutzung und Bodenschutz. URL http://www.lbeg.de/extras/nlfbook/html/nds_main.htm. Abgerufen am 09.07.2010.

NLWKN (NIEDERSÄCHSISCHER LANDESBETRIEB FÜR WASSERWIRTSCHAFT, KÜSTEN UND NATURschUTz) (2010a): NATURA 2000: Europäische Vogelschutzgebiete und gemeldete FFH-Gebiete in Niedersachsen. URL http:/ / www.umwelt.niedersachsen. de/portal/live.php?navigation_id=2540\&article_id=9124\&_psmand=10. Abgerufen am 10.09.2010.

NLWKN (NiEDERSÄCHSISCHER LANDESBETRIEb FÜR WASSERWIRTSCHAFT, KÜSTEN UND Naturschutz) (2010b): Naturschutzrechtlich besonders geschützte Teile von Natur und Landschaft in Niedersachsen. URL http://www.umwelt.niedersachsen. de/portal/live.php?navigation_id=2543\&article_id=9065\&_psmand=10. Abgerufen am 30.03.2010.

NMUK (Niedersächsisches Ministerium FÜR UMwelt ENERgIE UND KliMASchUTz) (2010): FFH-Richtlinie und gemeldete Gebiete. URL http: //www.mu.niedersachsen.de/live/live.php?navigation_id=2421\&article_id= 8632\&_psmand=10\&mode=print. Abgerufen am 20.09.2010. 
Otte, A.; Reger, B.; Simmering, D. \& Waldhardt, R. (2008): Prognose der Veränderungen von Phytodiversität in Agrarlandschaften. Berichte der Reinhold-TüxenGesellschaft 20: 67-89.

Pilgrim, B. \& Franke, R. (1993): Kartographische Arbeitsgrundlage für faunistische und floristische Erfassungen. Naturschutz und Landschaftspflege in Niedersachsen A/5. Niedersächsisches Landesamt für Ökologie.

PoHL, D. (1999): Die ältesten Naturschutzgebiete in Niedersachsen - eine chronologische Zusammenstellung. Informationsdienst Naturschutz Niedersachsen 3: 163-169.

R Development Core Team (2012): R: A language and environment for statistical computing. URL http://www.R-project.org.

Rodrigues, A. S. L.; Andelman, S. J.; Bakarr, M. I.; Boitani, L.; Brooks, T. M.; Cowling, R. M.; Fishrool, L. D.; Da Fonseca, G. A. B.; Gaston, K. J.; Hoffmann, M.; Long, J. S.; Marquet, P. A.; Pilgrim, J. D.; Pressey, R. L.; Schipper, J.; Sechrest, W.; Stuart, S. N.; Underhill, L. G.; Waller, R. W.; Watts, M. E. \& Yan, X. (2004): Effectiveness of the global protected area network in representing species diversity. Nature 428: 640-643.

Schmidt, M.; KRIEbitzsch, W.-U. \& EwAld, J. (2011): Waldartenlisten der Farn- und Blütenpflanzen, Moose und Flechten Deutschlands. BfN-Skripten 299: 1-111.

Schmidt, M.; Meyer, P.; PaAr, U. \& Evers, J. (2009): Bedeutung der Habitatkontinuität für die Artenzusammensetzung und -vielfalt der Waldvegetation. Forstarchiv 80: 195-202.

Schmiedel, I.; Schacherer, A.; Hauck, M.; Schmidt, M. \& Culmsee, H. (2011): Verbreitungsmuster der Farn- und Blütenpflanzen in Niedersachsen und Bremen unter Berücksichtigung ihres Einbürgerungsstatus und ihrer Gefährdungssituation. Tuexenia 31: 211-226.

SeKRETARIAT DER CBD (2007): Globale Strategie zur Erhaltung der Pflanzen: Übersetzung der englischen Originalfassung von April 2002. 15 S.

Ssymank, A. (1998): Das europäische Schutzgebietssystem NATURA 2000. Schriftenreihe für Landschaftspflege und Naturschutz 53: 1-560.

Vellak, A.; Tuvi, E.-L.; Reier, Ü.; Kalamees, R.; Roosaluste, E.; Zobel, M. \& Pärtel, M. (2009): Past and present effectiveness of protected areas for conservation of naturally and anthropogenically rare plant species. Conservation Biology 23: 750757. 


\section{The influence of landscape frag- mentation on richness patterns of vascular plants}

Schmiedel, I. \& Culmsee, H.: The influence of landscape fragmentation, expressed by the 'Effective Mesh Size Index', on regional patterns of vascular plant species richness in Lower Saxony, Germany. Submitted to journal. 


\section{Abstract}

Landscape fragmentation has been identified as a major threat to biodiversity worldwide. Several landscape metrics have been developed to quantify the extent of fragmentation, of which the Effective Mesh Size Index $\left(m_{e f f}\right)$ is one of the most widely used. However, its relevance for biodiversity has been rarely tested. We analysed the explanatory power of $m_{e f f}$ for richness patterns of different groups of vascular plant species (all species, and species groups by residence and threat status) in Lower Saxony, Germany, by using a grid of 1386 landscape units. Since we assumed species richness to be influenced by abiotic conditions and spatial autocorrelation, we used variation partitioning to separate the effects of these variables from that of fragmentation. We tested five types of $m_{e f f}$ based on various fragmentation geometries of which two were identified as relevant for species richness.

We found that $m_{\text {eff }}$ had a large effect on richness of neophytes and a slightly smaller effect on that of archaeophytes whilst the richness of native species was only slightly affected, and threatened species were not affected. All species groups, except threatened species, showed a negative correlation with $m_{e f f}$, i. e. richness was highest in highly fragmented and lowest in less fragmented grid cells.

We conclude that $m_{\text {eff }}$ is a meaningful tool to explain richness patterns of vascular plant diversity, if relevant fragmentation geometries are chosen. Our approach may therefore help future studies to determine correct fragmentation geometries to use with $m_{\text {eff }}$ and may facilitate the unravelling of fragmentation impacts on the landscape-scale.

\section{Keywords}

Alien plant species, archaeophytes, native plant species, naturalisation, neophytes, PCNM, Red List, regional plant species richness patterns, variation partitioning, vascular plant diversity 


\section{Introduction}

The term 'landscape fragmentation' describes both the process of the subdivision of a large continuous patch into smaller and more isolated fragments, as well as the state of a landscape (Forman, 1995). As soon as the effect of fragmentation on the biota is concerned, frequently also the term 'habitat fragmentation' is applied.

Besides general habitat loss and decline in habitat quality, fragmentation has been identified as a major threat to biodiversity worldwide (JAEgER, 2000; Young \& Clarke, 2000; Honnay et al., 2005; KuUssaAri et al., 2009; Krauss et al., 2010). In Central Europe, habitat change accelerated in course of urbanisation and industrialisation in the late 19th and early 20th century (TSCHARNTKE et al., 2005; KraUse \& CulmseE, 2013) and has been reinforced by the advent of the common agricultural policy of the EU since the 1950s (StOATE et al., 2001; KRAUSE et al., 2011; LeUsCHNER et al., 2013). However, landscape development in Central Europe currently does not appear to be following a clear trend, and we observe complex and diverse landscapes as a result of multi-layered historical and current land use practices (Vos \& MEEKEs, 1999; Culmsee, 2013). This, in turn, results in differences in the fragmentation intensity between neighbouring landscapes and in a more or less strong gradient of fragmentation on the regional and supra-regional scales.

The analysis of landscape fragmentation is an important aspect of landscape ecological research (HAILA, 2002). Many different indices for the quantification of landscape fragmentation have been introduced, such as the Number of Undissected Areas, Bowens Landscape Dissection Index (Bowen \& Burgess, 1981), Splitting Index (JAEgER, 2000) and Effective Mesh Size (JAEGER, 2000). All of these indices provide a spatially differentiated assessment of the fragmentation of a region (JAEGER, 2000). By evaluating these four plus additional four indices, JAEgER (2000, p. 127) found that Effective Mesh Size $\left(m_{e f f}\right)$ is the most appropriate fragmentation measure due to its "mathematical characteristics and its intuitive interpretation". The index quantifies the probability that two randomly chosen points in a study area are connected (GIRVETz et al., 2008) and has frequently been used to quantify landscape fragmentation, e. g. in Europe (EEA, 2011), Germany (JAEger et al., 2001; Esswein \& SchWARZ-von RAUMER, 2004; Walz, 2005; Esswein \& Schwarz-von Raumer, 2006), Italy (Moser et al., 2007), Switzerland (JAEger et al., 2008), the USA (GIRvetz et al., 2008) and China (Li et al., 2010).

In Germany, $m_{\text {eff }}$ is one of two indicators used by the government to quantify landscape fragmentation for environmental reporting (BMU, 2007). However, despite its frequent application and the fact that the ecological relevance of $m_{\text {eff }}$ has been proposed by many researchers (JAEGER, 2000; JAEGER et al., 2008; GIRVETz et al., 2008), its effect on biodiversity has been rarely analysed (but see LI et al., 2010). STRAND et al. (2007) suggested that studies investigating the direct effect of landscape fragmentation (expressed in terms of fragmentation metrics) on biodiversity should be undertaken, because indicators that quantify landscape fragmentation are more useful if they can be directly linked to possible impacts on species diversity and distributions. 
A multitude of man-made landscape elements, including urban, industrial and traffic infrastructure, can cause landscape fragmentation, resulting in patchy landscapes with generally small remaining natural or semi-natural habitat fragments. Depending on the specific biological traits of the species, very different landscape elements, including (near-) natural ones, can be considered fragmentation elements (see GIRVETZ et al., 2008; EEA, 2011).

The fragmentation of habitats may result in increasing isolation of plant or animal subpopulations that inhabit the patches. Habitats colonised by different subpopulations of the same species are isolated if genetic exchange is constrained because the area between the patches is too large, or too impermeable, to be overcome by pollination or dispersal events (Oostermeijer et al., 1994; Poschlod et al., 1997). The loss of genetic variation, in turn, may hamper the species' ability to respond to changing environmental conditions (Booy et al., 2000) and the populations may thus become threatened with extinction. Numerous studies examined the effect of habitat fragmentation on plant and animal assemblages at the local and landscape scales (see for an overview Debinski \& Holt, 2000; Fahrig, 2003). Frequently, the size of and the isolation of or connectivity between patches were used as measure of fragmentation (e.g. Helm et al., 2006; Cousins et al., 2007; KolB, 2008; Zimbres et al., 2013), yet none of these studies applied the Effective Mesh Size Index.

The effects of landscape fragmentation on species richness may vary depending on the species groups considered (EwERs \& DidHAM, 2006; Rodriguez-LoinAz et al., 2012). Certain species, particularly those spreading along linear landscape elements or associated with man-made habitats, may benefit from landscape fragmentation (Deutschewitz et al., 2003; Honnay et al., 2003; Dark, 2004; Nobis et al., 2009; ВотHAM et al., 2009). In contrast, threatened species may be adversely affected by fragmentation events (EwERs \& DidHAM, 2006). However, especially species that exhibit long life cycles and small population sizes show delayed extinctions following habitat loss and fragmentation (KUUSSAARI et al., 2009; KRAUSs et al., 2010), which at first can hamper the detection of these negative effects. This phenomenon is known as 'extinction debt' (Tilman et al., 1994). However, the evidence for the existence of an extinction debt in vascular plants across Europe is ambiguous (reviewed in Cousins, 2009).

The overall objective of our study was to investigate whether the frequently used Effective Mesh Size Index $\left(m_{e f f}\right)$ is a suitable measure to explain vascular plant species richness on the landscape scale. We used the state of Lower Saxony, Germany, as a model region as it hosts a high diversity of landscapes differing in habitat composition and structural complexity. Therefore, we expected a long gradient in fragmentation across the study area. Furthermore, the area is particularly suitable due to the availability of extensive data collected in a state-wide mapping program on the distribution of vascular plants with a resolution of c. $30 \mathrm{~km}^{2}$ (NLWKN 1982-2003, GARVE, 2007). Thus, high quality data were available for a region that 
is more or less homogeneous in climate and bio-geographical history, so that we considered the same regional species pool throughout the studied landscapes. We used different subsets of fragmentation geometries (FGs) and also different subsets of the regional plant species pool, dependent on the residence and threat status of the species, in order to investigate the underlying patterns in plant species distributions as responses to different fragmenting landscape elements.

Specifically, we hypothesised that:

1. Landscape fragmentation, expressed as Effective Mesh Size $\left(m_{\text {eff }}\right)$, varies in space, which results in a gradient in the degree of fragmentation within the sample of landscape units used in this study.

2. The choice of fragmentation geometries that are used for the calculation of $m_{e f f}$ is of major importance for explaining plant species richness patterns on the landscape scale.

3. Plant species richness varies between landscape units along the fragmentation gradient, with the effect size differing between different groups of species dependent on their residence (natives, archaeophytes and neophytes) and threat status (threatened vs. non-threatened).

\section{Methods}

\section{Study area}

The study was carried out in Lower Saxony, Germany (c. $47,500 \mathrm{~km}^{2}$, Fig. 5.1). As a result of the variety in relief and bedrock, colonisation history and current land use are diverse across north-western Germany (BEHRE, 2008). Lower Saxony harbours a relatively large number of different habitats, which include natural coastal and forest-dominated landscapes, structured and homogeneous agricultural landscapes, and densely populated areas (GHARADJEDAGHI et al., 2004).

The coastal area in the north is dominated by Holocene marine deposits, while the lowlands were formed during the Pleistocene. The landscape of the uplands is shaped by Mesozoic and Palaeozoic bedrocks. From the coast in the north to the uplands in the southeast, elevation ranges from 0-971 $\mathrm{m}$ a.s.l.. The climate gradually changes eastwards from oceanic (mean annual precipitation up to $900 \mathrm{~mm}$ ) to suboceanicsubcontinental (c. $550 \mathrm{~mm}$; NLfB, 1997).

Following the grid system of topographical maps, the study area was subdivided into a regular grid of 1386 cells sized c. $5.5 \times 5.5 \mathrm{~km}^{2}\left(30 \mathrm{~km}^{2}\right.$; Fig. 5.1). In our analysis, we omitted the 353 border cells only partially occurring in the study area. 


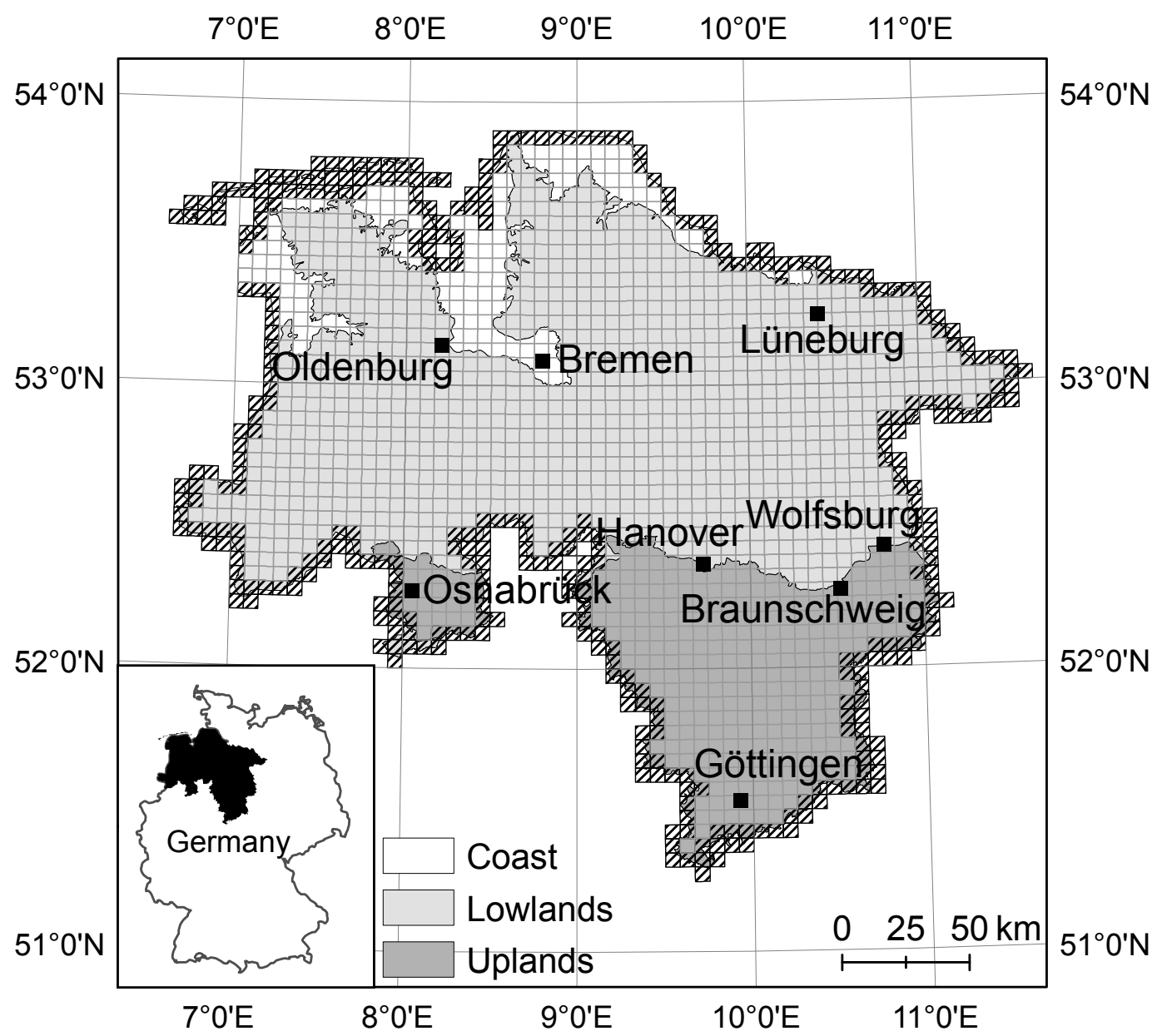

Figure 5.1: The state of Lower Saxony, Germany, with its three major landscape components: coast, lowlands and uplands. The 1386 grid cells (size c. $5.5 \times 5.5 \mathrm{~km}^{2}$ or $30 \mathrm{~km}^{2}$ ) display the basic mapping units of the vascular plant survey program and represent the landscape units for which landscape fragmentation was assessed. Hatched grid cells were not considered in this study, because they are not completely terrestrial or they are situated at the state's borders and thus information on plant occurrences were incomplete. Maps with Transverse Mercator projection, Germany Zone 3 ("Deutsches Hauptdreiecksnetz").

\section{Plant species distribution data}

Species occurrences were derived from the data base of the plant survey of the state of Lower Saxony (NLWKN, Hanover, Germany; period I from 1982 to 1992; period II from 1993 to 2003; period III from 2004 onwards). As the third fieldwork period is still underway, we used only data from 1982-2003 (GARVE, 2007; data base updated on 15 December 2008). The data base holds information about the presence and distribution of 2708 vascular plant species occurring in Lower Saxony (GARvE, 2007). 
Table 5.1: Number of vascular plant species in Lower Saxony, Germany, grouped by their residence and threat status.

\begin{tabular}{|c|c|c|c|c|c|}
\hline & & \multicolumn{3}{|c|}{ Threat sta tus } & \multirow[t]{2}{*}{$\begin{array}{l}\text { Sum of resi- } \\
\text { dence status }\end{array}$} \\
\hline \multirow{6}{*}{$\begin{array}{l}\text { Residence } \\
\text { status }\end{array}$} & & Non-threatened & Threatened & Unspecified & \\
\hline & Native species & 703 & 482 & 112 & 1297 \\
\hline & Archaeophytes & 103 & 46 & 0 & 149 \\
\hline & Neophytes & 134 & 9 & 0 & 143 \\
\hline & Unspecified & 4 & 1 & 0 & 5 \\
\hline & Sum of threat status & 944 & 538 & 112 & 1594 \\
\hline
\end{tabular}

Species were assigned to different groups according to their residence and threat status (Table 5.1). In the designation of the residence status of the plant species we followed KLOTz et al. (2002). Native species were distinguished from archaeophytes and neophytes. Within the non-native species, archaeophytes became established prior to and neophytes in the $16^{\text {th }}$ century or later. In the designation of threat status we followed GARve (2004), whereby threatened species (including the Red List categories 1, $2,3, \mathrm{G}$ and R) were distinguished from non-threatened species. Five species could not be assigned to a certain floristic status, while 112 species lacked information about their threat status (hereafter 'not specified' species). Subspecies were assigned to the species level and species to the respective aggregate found in the data base. In total, 1594 species remained that were considered in the analysis.

For each species group, species richness (based on presence/absence data) was calculated for each of the 1386 grid cells. Distribution patterns of the selected species groups were described in detail by SCHMIEDEL et al. (2011).

\section{Quantification of landscape fragmentation}

Landscape fragmentation analysis was conducted with the 1386 grid cells used by the plant species monitoring program (Fig. 5.1). Each grid cell was considered a landscape unit. Landscape elements were derived from a digital landscape model (DLM) of Lower Saxony, scale 1:25,000 (LGN, 2008), and summarised to eight major habitat types that represent suitable habitat formations for vascular plants: forest, grassland, cropland, water bodies, urban, industrial and traffic areas, and other habitats (e.g. islands, tree rows, bogs). Linear elements represented in the DLM as polylines (roads, rivers) were buffered by using either standard values (roads; FGSV, 1996) or information provided by the dataset itself (rivers) in order to convert them to polygons. Data were converted by using Hawth's Analysis Tools (BEYER, 2004) and combined with the other polygon features to a non-overlapping feature layer by using ArcGISArcInfo 9.2 (ESRI INC., 2007). 
Habitat types that were assumed to increase fragmentation of habitats suitable for different groups of vascular plant species were classified into five types of fragmentation geometries (FG1-FG5; Table 5.2). Starting with FG1, fragmenting landscape elements were successively added to the following FGs. FG1 included the most artificial land cover classes of traffic and urban-industrial areas. FG2 additionally included water bodies. Similar FGs have frequently been used in fragmentation studies and will thus allow for comparisons. The subsequent FG3-FG5 were built by adding further land cover classes (FG3: croplands, FG4: grasslands, FG5: other habitats) to the former FGs, thus setting a gradient of decreasing human impact (Fig. 5.2). FG5 included all landscape elements except forests which were rated as (semi-)natural landscape elements, because forests represent the potential natural vegetation of temperate Europe (BoHN \& NeuHäUsL, 2000/2003).

Landscape fragmentation was quantified by the Effective Mesh Size Index $\left(m_{\text {eff }}\right)$. The index denotes the size of patches when the landscape is divided into $S$ areas (each of the same size) with the same degree of landscape division as obtained for the observed cumulative area distribution (McGarigal, 2014). Thus $m_{\text {eff }}$ is an ecologically relevant metric that quantifies landscape fragmentation based on the probability that two randomly chosen points in a landscape unit are located in the same nonfragmented patch (JAEGER, 2000). The probability is multiplied by the total area of the landscape unit (in $\mathrm{m}^{2}$ ) and finally gives the value of $m_{\text {eff }}$. The Effective Mesh Size Index was calculated following McGARIGAL (2014) using the following formula:

$$
m_{e f f}=\frac{\sum_{j=1}^{n} a_{i j}^{2}}{A}\left(\frac{1}{10,000}\right)
$$

where $n$ is the number of patches, $a_{i j}$ is the size of patch $i j$ and $A$ is the total area of the landscape. Values of $m_{e f f}$ are given in hectares (ha) by using the factor of 1:10,000 to convert $\mathrm{m}^{2}$ into ha.

For the calculation of $m_{\text {eff }}$ based on different FGs, subsequently called MeshFG1MeshFG5 (Table 5.2), the FGs derived from the DLM vector data were transformed to raster format, and $m_{e f f}$ of the non-fragmented parts of the landscape was calculated for each grid cell using Fragstats 3.3 (McGarigal et al., 2002). We applied the CUT procedure proposed by JAEGER (2000), and not the CBC procedure as proposed by Moser et al. (2007), to obtain spatially explicit values for $m_{e f f}$ that could be correlated with the grid-based distribution data on plant species.

A Shapiro-Wilk normality test was performed on all $m_{\text {eff }}$ variables to test them for normal distribution with the $\mathrm{R}$ software, version 2.15.2 (R Development CoRe Team, 2012). Furthermore, a Wilcoxon signed-rank test was used to test for significant differences between the $m_{e f f}$ types. 

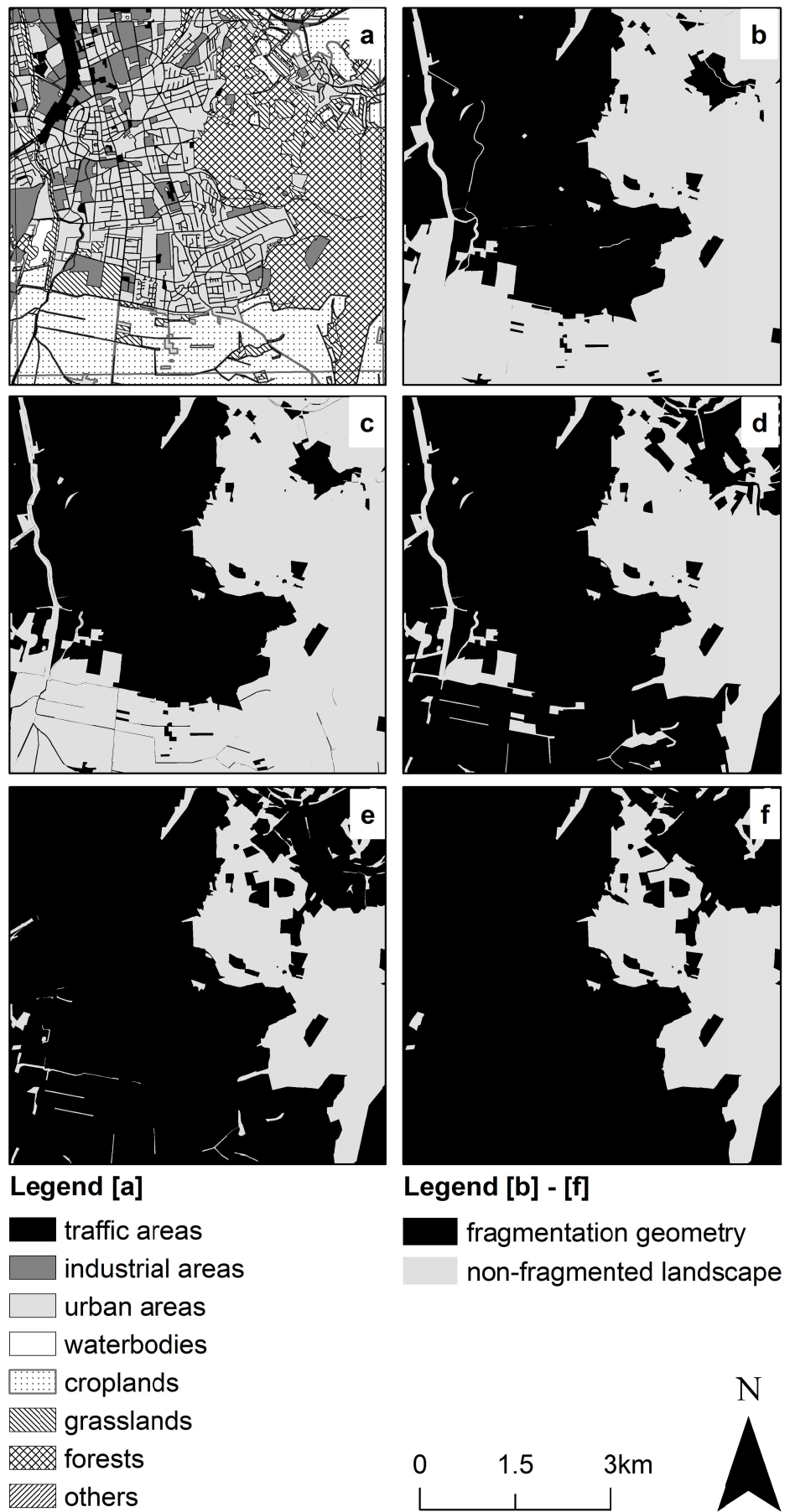

non-fragmented landscape

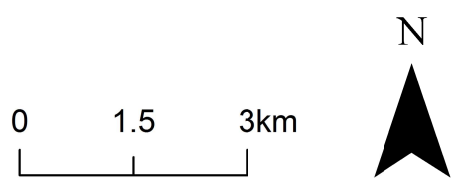

Figure 5.2: Example of one of 1386 landscape units in Lower Saxony, Germany (grid cell no. 44254 , Göttingen, sized c. $5.5 \times 5.5 \mathrm{~km}^{2}$ or $30 \mathrm{~km}^{2}$ ). (a) The digital landscape model (ATKISDLM, scale 1:25,000; LGN, 2008) was translated to five different fragmentation geometries, FG1-5, (b) FG1 (traffic and urban-industrial areas), (c) FG2 (FG1 + water bodies), (d) FG3 (FG2 + croplands), (e) FG4 (FG3 + grassland), (f) FG5 (all landscape elements except forested areas). 


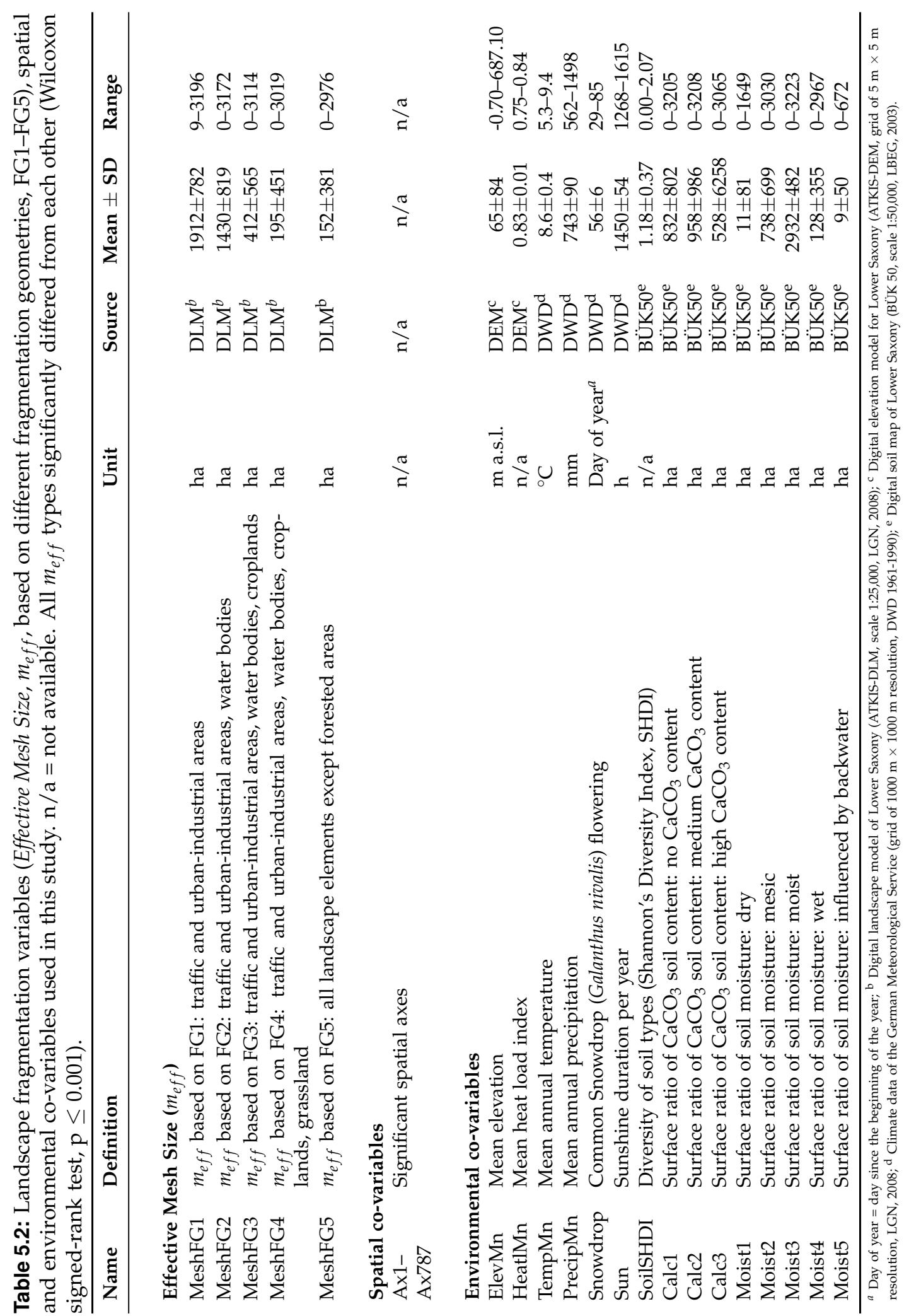




\section{Accounting for environmental co-variables and spatial autocorrelation}

We assumed that both landscape fragmentation and environmental factors influence species richness. Thus, to account for the explanatory value of the abiotic environment, supplementary environmental data were derived from various digital data sources (Table 5.2). Mean elevation was taken from the digital elevation model (DEM) of Lower Saxony (LGN, 2008). DEM data were further used to calculate mean heat load (McCune \& Keon, 2002, eq. 3) per grid cell by using the ArcScript 'Heat load index' (PARKs, 2004) in ArcView GIS 3.3 (ESRI INC., 2002). Information on soil moisture, $\mathrm{CaCO}_{3}$ content of soils and soil types were derived from the digital soil map of Lower Saxony (BÜK 50, LBEG, 2003). For each grid cell, the surface ratio of five soil moisture and three $\mathrm{CaCO}_{3}$ soil content classes were calculated. Based on the distribution and cover of 27 soil types, for each grid cell the Shannon Diversity Index (SHDI) was computed in Fragstats 3.3 after vector data were transformed to raster format. Information on climatic and phenological conditions (annual mean precipitation and temperature, start of snowdrop flowering) per grid cell were derived from digital data of the German Meteorological Service (DWD, 1961-1990) and the average of each variable was calculated per grid cell. The 15 environmental variables that were used in the analysis were uncorrelated (Spearman's rank correlation coefficient $(|\rho| \leq 0.7)$.

To account for spatial autocorrelation in the dataset, PCNM technique was applied using the following steps (cp. BorCARD \& LEgEndre, 2002; Borcard et al., 2004): (1) We constructed a matrix of Euclidean distances between the 1386 grid cells (landscape units) based on the $x$ - and y-coordinates (centroids) of the cells. (2) We defined a threshold (smallest distance keeping all cells connected, i. e. $8036 \mathrm{~m}$ ) below which the Euclidean distances were kept as measures and above which all distances were replaced by a value four times larger than the threshold (i. e. 32,144 m). (3) We computed the principal coordinates (correction of negative eigenvalues) of the modified matrix using PrCoord, version 1.0 (implemented in CANOCO 4.5, BRAAK \& ŠMILAUER, 2002), and (4) we reduced the number of the remaining 787 spatial variables to those that were significant for the richness of the species groups (Monte Carlo Permutation test under full model, 99,999 permutations; Holm-correction, $\mathrm{p} \leq 0.05$; LEGENDRE \& LEGENDRE, 2012).

\section{Statistical analysis}

In order to determine the explanatory power of $m_{e f f}$, the environmental factors and spatial axes for species richness patterns across the 1386 grid cells, variation partitioning was applied by using the 'varpart' function ('vegan' package, OKSANEN et al., 2012) in R (cp. Fig. 5.3). Separate analyses were conducted for each species group (Table 5.1) in combination with each type of $m_{\text {eff }}$ (Table 5.2). Species numbers of threatened species were square root transformed because they showed a 
skewed distribution. Significance of all testable fractions was tested using 'rda' and 'anova.cca' functions in 'vegan'.

To determine the direction of the relationship of species richness and the $m_{\text {eff }}$ types, Kendall's $\tau$ was calculated using R. This correlation analysis was performed for MeshFG1 and MeshFG2 only because variation partitioning showed that the other types of $m_{e f f}$ explained only a negligible proportion of the species richness of all groups.

\section{Results}

\section{Variation in Effective Mesh Size ( $m_{\text {eff }}$ ) based on different fragmentation geometries across landscapes}

Values of $m_{e f f}$ calculated for the 1386 landscape units (grid cells) across the study area showed large variation both in range between landscapes and between fragmentation geometries (Table 5.2). All $m_{\text {eff }}$ types (MeshFG1-MeshFG5) showed a large range from entirely fragmented $\left(m_{\text {eff }}=0 \mathrm{ha}\right)$ to entirely non-fragmented ( $m_{\text {eff }} \sim 3,000 \mathrm{ha}$ ) landscape units, the latter corresponding to the total size of a landscape unit of c. $30 \mathrm{~km}^{2}$. The mean value of $m_{e f f}$ was highest when only traffic and urban-industrial areas were used (MeshFG1, $m_{e f f}=1,912 \mathrm{ha} \pm 782 \mathrm{SD}$ ) indicating a relatively low fragmentation impact, and was lowest when all landscape elements with the exception of forests were used (MeshFG5, $m_{\text {eff }}=152$ ha \pm 381 SD). From MeshFG1 to MeshFG5, mean $m_{\text {eff }}$ gradually decreased when additional fragmentation geometries were successively included, whereas each of them had an additional impact as fragmenting landscape element against the previous (Wilcoxon signed-rank test, $\mathrm{p}<0.001$ for all pairs of MeshFG1-MeshFG5). None of the $m_{e f f}$ variables was normally distributed (Shapiro-Wilk normality test, $\mathrm{p} \leq 0.05$ ). Values of MeshFG1 showed a slightly rightskewed distribution, i. e. larger values of $m_{\text {eff }}$ were more frequent than small values (Fig. 5.4a), while values of $m_{e f f}$ for MeshFG2 were slightly left-skewed (Fig. 5.4b).

Figure 5.3: Fractions obtained from variation partitioning based on three variables (Venn diagram). Fractions [a] to [c] correspond to the pure effect of variables $a, b$ and $c$, fractions [d] to [f] correspond to the variation explained by variables $a$ and $b, b$ and $c$, and a and $\mathrm{c}$, respectively. Fraction [g] corresponds to the variation explained by all three variables together whereas [h] describes the unexplained variance (residuals).

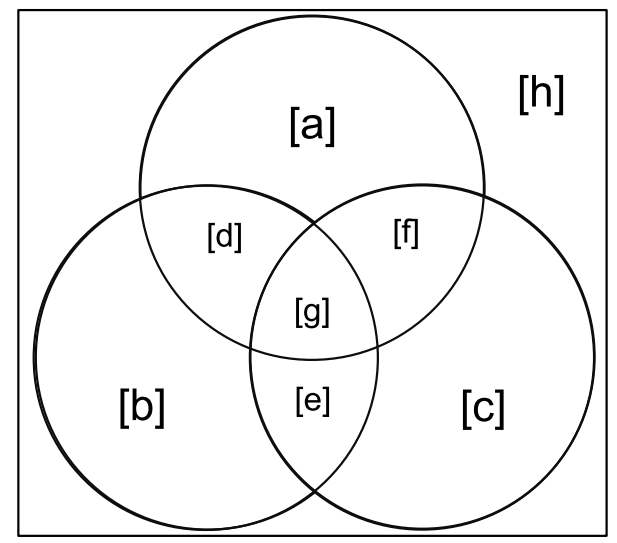


The remaining $m_{e f f}$ types (Fig. 5.4c-e) all showed a strongly left-skewed distribution with the majority of landscape units exhibiting a mesh size $<200$ ha.
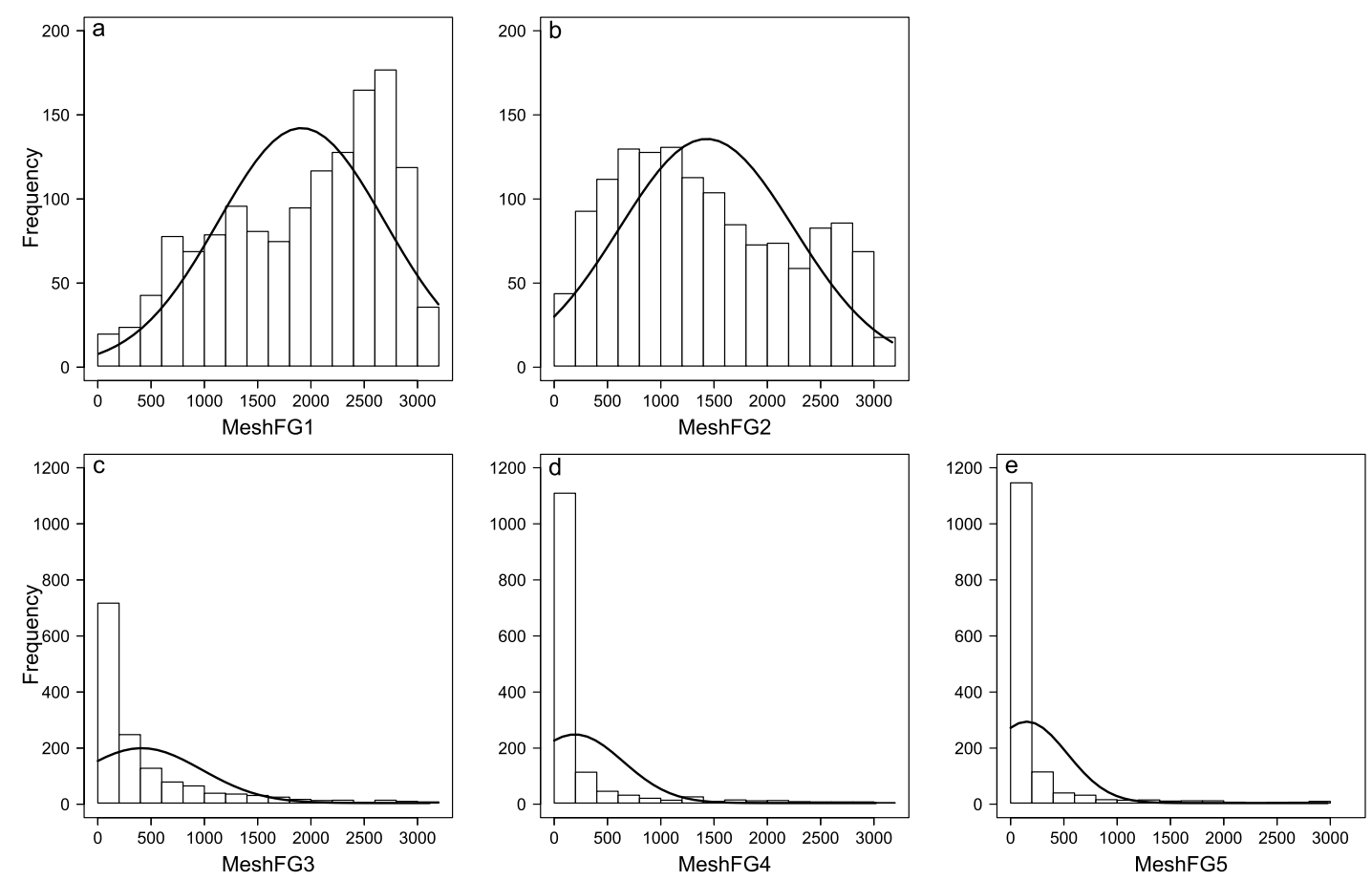

Figure 5.4: Frequency distribution of the five $m_{\text {eff }}$ types (a-e, MeshFG1-MeshFG5) for the sample of 1386 landscape units (grid cells) in Lower Saxony, Germany. Maximum value of $m_{\text {eff }}$ was c. 3,000 ha which corresponds to the size of a fully non-fragmented landscape unit of $30 \mathrm{~km}^{2}$. None of the distributions were normally distributed (Shapiro-Wilk normality test, $\mathrm{p} \leq 0.05)$.

\section{The explanatory power of Effective Mesh Size $\left(m_{\text {eff }}\right)$, based on different fragmentation geometries, versus other environmental factors for species richness-patterns on the landscape-scale}

Depending on fragmentation geometries (FGs) used for the calculation of $m_{e f f}$ and on the species group considered, the variation partitioning revealed large differences in the explanatory power of $m_{\text {eff }}$ versus other environmental factors for vascular plant species richness patterns on the landscape scale (Table 5.3).

Accounting for the total of 1594 plant species, environmental variables explained $14 \%( \pm 0 \mathrm{SD})$ and spatial variables $7 \%( \pm 0 \mathrm{SD})$ of the variance in species richness (Table 5.3a). The response to landscape fragmentation largely varied in dependence on the mesh size variable (MeshFG1-MeshFG5). While $m_{e f f}$ types that used only traffic, urban-industrial areas and water bodies had a considerable influence of $5 \%$ (MeshFG1) and $6 \%$ (MeshFG2), the other $m_{e f f}$ variables, which additionally included 
other man-made habitats such as agricultural areas and grasslands (MeshFG3 MeshFG5), influenced species richness by $<1 \%$. Similar patterns were observed across all species groups. On average, $52 \%( \pm 3 \mathrm{SD})$ of variation in all species remained unexplained, and similar figures were found in the other species groups, with a range of $42 \%( \pm 4 \mathrm{SD})$ of unexplained variation in archaeophytes (Table 5.3c) to $61 \%( \pm 6 \mathrm{SD})$ in threatened species (Table 5.3f).

When distinguishing species groups according to the residence status, the general patterns observed in all species were more or less repeated in the partition of the largest group of 1297 native species, but with slightly lower explanatory power of the $m_{e f f}$ variables (Table 5.3b). Spatial distribution patterns of natives were also relatively clustered (explanation of the spatial fraction was $8 \% \pm 0 \mathrm{SD}$ ). However, the smaller groups of archaeophytes (149 sp.) and neophytes (143 sp.) showed different patterns. Archaeophytes were more strongly related to landscape fragmentation (MeshFG1 and MeshFG2: $7 \%$ ) and especially to environmental conditions (26\% \pm 2 SD; Table $5.3 \mathrm{c})$, indicating a relatively narrow environmental niche width of these species. Neophytes were most strongly associated with fragmented landscapes (Table 5.3d). $M_{\text {eff }}$ explained a large proportion of species richness, namely 15\% (MeshFG1) and 16\% (MeshFG2), while the environmental factors were of lower importance $(10 \% \pm 2 \mathrm{SD})$. Spatial autocorrelation was generally low in both non-native groups ( $<2 \%$ of the total explained variation; Table 5.3c and d).

With respect to threat status, the large group of 944 non-threatened species followed the patterns observed in all species (Table 5.3e). In contrast, threatened species showed spatially distinguished incidence patterns (explained variation of spatial axes: $7 \% \pm 0 \mathrm{SD}$ ) and their occurrences were mostly determined by environmental variables $(19 \% \pm 1 \mathrm{SD})$, while they were not affected $\left(m_{\text {eff }}<1 \%\right)$ by landscape fragmentation (Table 5.3f).

With the exception of the threatened species group that showed no specific trend, all species groups were negatively correlated with MeshFG1 and MeshFG2 (Table 5.4), which means that species richness increased with increasing landscape fragmentation (i.e. small $m_{\text {eff }}$ values).

\section{Discussion}

\section{Variation in landscape fragmentation based on $\boldsymbol{m}_{\text {eff }}$}

The values of $m_{e f f}$ obtained for the 1386 landscape units in our study varied between 0 and $30 \mathrm{~km}^{2}$. Thus, we identified a large gradient from entirely fragmented to entirely non-fragmented landscape units. Because we applied the CUT procedure to calculate $m_{\text {eff }}$, maximum values of the index were restricted by the size of the study units. The latter also applies to other studies on $m_{\text {eff }}$ (see Table 5 for an overview), which used differently sized reference units for the calculation of the index. Therefore, conclusions from comparisons of our results with values of $m_{\text {eff }}$ obtained in these 
Table 5.3: Results of variation partitioning (explained variation) for vascular plant species richness in Lower Saxony, Germany, in the groups of (a) all species, (b) natives, (c) archaeophytes, (d) neophytes, (e) non-threatened species, and (f) threatened species (numbers given in brackets), in relation to landscape fragmentation expressed as Effective Mesh Size (MeshFG1MeshFG5) based on different sets of fragmentation geometries (FG1-FG5), spatial axes as measures for spatial autocorrelation and environmental factors (cp. Table 5.2). Significance was tested by Monte Carlo Permutation test (999 permutations) and refers to the partitions [a], [b] and [c] (cp. Fig. 5.3); significant results are given in bold letters; ${ }^{* *}, \mathrm{p} \leq 0.001$; ${ }^{* *}$ $\mathrm{p} \leq 0.01, *, \mathrm{p} \leq 0.05$.

\begin{tabular}{|c|c|c|c|c|c|}
\hline & MeshFG1 & MeshFG2 & MeshFG3 & MeshFG4 & MeshFG5 \\
\hline \multicolumn{6}{|l|}{ (a) All species (1594 sp.) } \\
\hline [a] $M_{\text {eff }}$ & $0.048^{* * *}$ & $0.061^{* * *}$ & $0.004 * *$ & $0.004 * *$ & $0.007 * * *$ \\
\hline [b] Spatial axes & $0.067 * * *$ & $0.067 * * *$ & $0.073 * * *$ & $0.071 * * *$ & $0.074 * * *$ \\
\hline [c] Environmental variables & $0.125 * * *$ & $0.145 * * *$ & $0.131 * * *$ & $0.139 * * *$ & $0.141 * * *$ \\
\hline Shared variation $[\mathrm{d}],[\mathrm{e}],[\mathrm{f}],[\mathrm{g}]$ & 0.264 & 0.242 & 0.252 & 0.245 & 0.241 \\
\hline [h] Residuals & 0.497 & 0.484 & 0.541 & 0.540 & 0.537 \\
\hline \multicolumn{6}{|l|}{ b) Natives (1297 sp.) } \\
\hline [a] $M_{\text {eff }}$ & $0.024 * * *$ & $0.037 * * *$ & 0.001 & $0.002 *$ & $0.005 * * *$ \\
\hline [b] Spatial axes & $0.073 * * *$ & $0.073 * * *$ & $0.08 * * *$ & $0.077 * * *$ & $0.079 * * *$ \\
\hline [c] Environmental variables & $0.121 * * *$ & $0.138 * * *$ & $0.123 * * *$ & $0.126 * * *$ & $0.129 * * *$ \\
\hline Shared variation $[\mathrm{d}],[\mathrm{e}],[\mathrm{f}],[\mathrm{g}]$ & 0.261 & 0.243 & 0.252 & 0.251 & 0.247 \\
\hline [h] Residuals & 0.521 & 0.509 & 0.544 & 0.543 & 0.540 \\
\hline \multicolumn{6}{|l|}{ (c) Archaeophytes (149 sp.) } \\
\hline [a] $M_{\text {eff }}$ & $0.067 * * *$ & $0.069 * * *$ & $0.013 * * *$ & $0.011 * * *$ & $0.011 * * *$ \\
\hline [b] Spatial axes & $0.011 * * *$ & $0.016 * * *$ & $0.018 * * *$ & $0.018 * * *$ & $0.019 * * *$ \\
\hline [c] Environmental variables & $0.245 * * *$ & $0.269 * * *$ & $0.247 * * *$ & $0.276 * * *$ & $0.274 * * *$ \\
\hline Shared variation $[\mathrm{d}],[\mathrm{e}],[\mathrm{f}],[\mathrm{g}]$ & 0.282 & 0.252 & 0.272 & 0.244 & 0.244 \\
\hline [h] Residuals & 0.397 & 0.394 & 0.450 & 0.453 & 0.452 \\
\hline \multicolumn{6}{|l|}{ (d) Neophytes (143 sp.) } \\
\hline [a] $M_{e f f}$ & $0.154 * * *$ & $0.158^{* * *}$ & $0.010 * * *$ & $0.005 * * *$ & $0.002 *$ \\
\hline [b] Spatial axes & $0.010 * * *$ & $0.016 * * *$ & $0.021 * * *$ & $0.022 * * *$ & $0.023 * * *$ \\
\hline [c] Environmental variables & $0.078 * * *$ & $0.078 * * *$ & $0.095 * * *$ & $0.115 * * *$ & $0.115 * * *$ \\
\hline Shared variation $[\mathrm{d}],[\mathrm{e}],[\mathrm{f}],[\mathrm{g}]$ & 0.152 & 0.147 & 0.124 & 0.103 & 0.103 \\
\hline [h] Residuals & 0.606 & 0.602 & 0.750 & 0.755 & 0.758 \\
\hline \multicolumn{6}{|c|}{ (e) Non-threatened species (944 sp.) } \\
\hline [a] $M_{\text {eff }}$ & $0.059 * * *$ & $0.069 * * *$ & $0.007 * * *$ & $0.006 * * *$ & $0.007 * * *$ \\
\hline [b] Spatial axes & $0.063 * * *$ & $0.064 * * *$ & $0.071 * * *$ & $0.068 * * *$ & $0.072 * * *$ \\
\hline [c] Environmental variables & $0.113 * * *$ & $0.135 * * *$ & $0.119 * * *$ & $0.13^{* * *}$ & $0.132 * * *$ \\
\hline Shared variation $[\mathrm{d}],[\mathrm{e}],[\mathrm{f}],[\mathrm{g}]$ & 0.275 & 0.252 & 0.262 & 0.252 & 0.248 \\
\hline [h] Residuals & 0.490 & 0.479 & 0.542 & 0.543 & 0.541 \\
\hline \multicolumn{6}{|l|}{ (f) Threatened species (538 sp.) } \\
\hline [a] $M_{\text {eff }}$ & $0.002 *$ & $0.008 * * *$ & $0.004 * *$ & 0.000 & 0.001 \\
\hline [b] Spatial axes & $0.066 * * *$ & $0.066^{* * *}$ & $0.068 * * *$ & $0.067 * * *$ & $0.066^{* * *}$ \\
\hline [c] Environmental variables & $0.191 * * *$ & $0.197 * * *$ & $0.180 * * *$ & $0.174 * * *$ & $0.185 * * *$ \\
\hline Shared variation $[\mathrm{d}],[\mathrm{e}],[\mathrm{f}],[\mathrm{g}]$ & 0.090 & 0.084 & 0.100 & 0.107 & 0.096 \\
\hline [h] Residuals & 0.650 & 0.644 & 0.648 & 0.652 & 0.651 \\
\hline
\end{tabular}


Table 5.4: Correlation of the $m_{e f f}$ types MeshFG1 and MeshFG2, respectively, with species richness of six species groups (all species, groups after naturalization or threat status) assessed for Lower Saxony, Germany, using Kendall's $\tau(\mathrm{p} \leq 0.05)$ as correlation coefficient.

\begin{tabular}{llrrr}
\hline$m_{\text {eff }}$ type & Species group & Kendall's $\tau$ & z-score & p-value \\
\hline \multirow{4}{*}{$\begin{array}{l}\text { MeshFG1 } \\
\text { max =3,196 ha, min }\end{array}$} & All species & -0.188 & -10.49 & $<2.2 \mathrm{e}-16$ \\
$=9.3 \mathrm{ha})$ & Natives & -0.137 & -7.62 & $2.61 \mathrm{E}-14$ \\
& Archaeophytes & -0.246 & -13.62 & $<2.2 \mathrm{e}-16$ \\
& Neophytes & -0.327 & -18.00 & $<2.2 \mathrm{e}-16$ \\
& Non-threatened species & -0.211 & -11.75 & $<2.2 \mathrm{e}-16$ \\
& Threatened species $^{a}$ & -0.015 & -0.84 & 0.4016 \\
& & & & \\
MeshFG2 & All taxa & -0.164 & -9.15 & $<2.2 \mathrm{e}-16$ \\
$(\mathrm{max}=3,172 \mathrm{ha}, \mathrm{min}$ & -0.109 & -6.04 & $1.55 \mathrm{E}-09$ \\
$=0$ ha) & Natives & -0.218 & -12.07 & $<2.2 \mathrm{e}-16$ \\
& Archaeophytes & -0.343 & -18.84 & $<2.2 \mathrm{e}-16$ \\
& Neophytes & -0.183 & -10.19 & $<2.2 \mathrm{e}-16$ \\
& Non-threatened species & -0.011 & -0.62 & 0.5364 \\
\hline
\end{tabular}

${ }^{a}$ Species richness of threatened species was square root transformed because it showed a skewed distribution.

studies have to be drawn with care. In contrast to the studies listed in Table 5, in our analysis, we applied units of equal size and thus the values of $m_{\text {eff }}$ may be compared more easily. However, as we used relatively small landscape units, the actual degree of fragmentation may be overestimated. Nevertheless, compared to the results of other studies on $m_{e f f}$ in Germany (Table 5, no. 3-5), all of which used differently sized study units, our findings showed a similarly large variation. Values of $m_{\text {eff }}$ obtained for Baden-Württemberg and Hesse ranged between 0 and c. $70 \mathrm{~km}^{2}$ (Jaeger et al., 2001; Esswein \& Schwarz-von Raumer, 2004; Table 5, no. 3 and 5). In Bavaria, the maximum mesh size was c. $300 \mathrm{~km}^{2}$, which was mainly a result of the high values of $m_{\text {eff }}$ obtained for largely non-fragmented study units situated at the foothills of the Alps (Esswein \& Schwarz-von Raumer, 2006; Table 5, no. 4). The influence of largely non-fragmented mountainous areas also becomes obvious if values of $m_{e f f}$ for Switzerland are considered: When large alpine areas were included into the calculation procedure, maximum values of $m_{e f f}$ exceeded $1,500 \mathrm{~km}^{2}$ (JAEGER et al., 2008; Table 5, no. 8). However, as soon as these uninhabitable areas were excluded from the computation process, the maximum values of $m_{e f f}$ dropped down to 300 to $700 \mathrm{~km}^{2}$.

The European Environment Agency (EEA, 2011; Table 5, no. 2) compared the $m_{\text {eff }}$ values of $28 \mathrm{EU}$ countries and came to the conclusion that Germany, following the Benelux countries, was one of the most fragmented countries in Europe. Thus, the relatively small values of $m_{e f f}$ obtained for most of our studied landscape units seem to be representative for most parts of Germany. In contrast, studies from outside densely populated Europe showed much larger maximum values of $m_{\text {eff }}$ (see Table 5, no. 1 and 9). For California, GIRvetz et al. (2008) who used fragmentation geometries 
similar to ours, and for China, LI et al. (2010) who calculated the Effective Mesh Size for countries and provinces, came up with maximum values of $m_{e f f}$ that exceeded these of our study a hundred- or even thousand-fold. However, even for these countries, the range of values of $m_{e f f}$ varied considerably: Whilst maximum values of $m_{e f f}$ in California reached c. 20,000 $\mathrm{km}^{2}$ (Table 5, no. 9, B.1), the smallest values obtained were equal to zero. Similarly, values of $m_{\text {eff }}$ for the 2,427 counties of China spanned a large range of c. $10 \mathrm{~km}^{2}$ (the most densely populated areas) to c. $680,000 \mathrm{~km}^{2}$ (the Tibetan plateau).

\section{The response of species richness patterns to $\boldsymbol{m}_{\text {eff }}$ as a function of the choice of fragmentation geometries}

Despite the fact that the FGs used in our analyses were nested, all types of $m_{\text {eff }}$ (MeshFG1-MeshFG5) significantly differed from each other and thus did not contain any redundant information. Nevertheless, when relating the values of $m_{\text {eff }}$ to species richness of the studied species groups, only MeshFG1 and MeshFG2 were found to be relevant. These $m_{\text {eff }}$ types are based on FGs that include urban-industrial and traffic areas as well as water bodies and have been frequently used in studies on landscape fragmentation (cp. Table 5). Thus, even without examining the relationship of $m_{\text {eff }}$ and species richness, many authors have chosen FGs that are meaningful for biodiversity. The explanatory power of MeshFG2 compared to that of MeshFG1 was slightly higher for all species groups. The inclusion of water bodies into the FGs used to calculate $m_{e f f}$ seems thus reasonable not only regarding animals, as proposed by GIRVETZ et al. (2008) and JAEGER et al. (2008), but also when distribution patterns of vascular plants are investigated. The remaining types of $m_{e f f}$ that included additional land cover classes supposed to have an added effect on the fragmentation of the landscape, did not explain (or only explained to a small extent) plant species richness patterns. We suppose that this was caused by the lack of a gradient of $m_{\text {eff }}$ in MeshFG3 - MeshFG5; for the majority of grid cells very low values of $m_{e f f}$ were reported and the frequency distribution thus was extremely skewed to the left. This results from a country-wide intensive alteration of landscapes in Lower Saxony in the course of human land use that left only very few (almost) completely forested landscapes. Similar results could possibly be obtained for other intensively used landscapes all over Europe. However, if landscapes with a larger amount of (semi)natural land cover classes are considered, the inclusion of agricultural areas (arable fields, managed grasslands) into $m_{\text {eff }}$ calculation may reveal a more differentiated picture and thus may be more suitable to explain plant species richness. In contrast to our results, GIRvETz et al. (2008) who investigated the effect of the inclusion of agricultural areas into the calculation of $m_{e f f}$ in California found these areas to only affect the degree of fragmentation in locations where extensive agricultural areas were present. In conclusion, the determination of relevant FGs is an important aspect when the relationship of landscape fragmentation and biodiversity is analysed. 


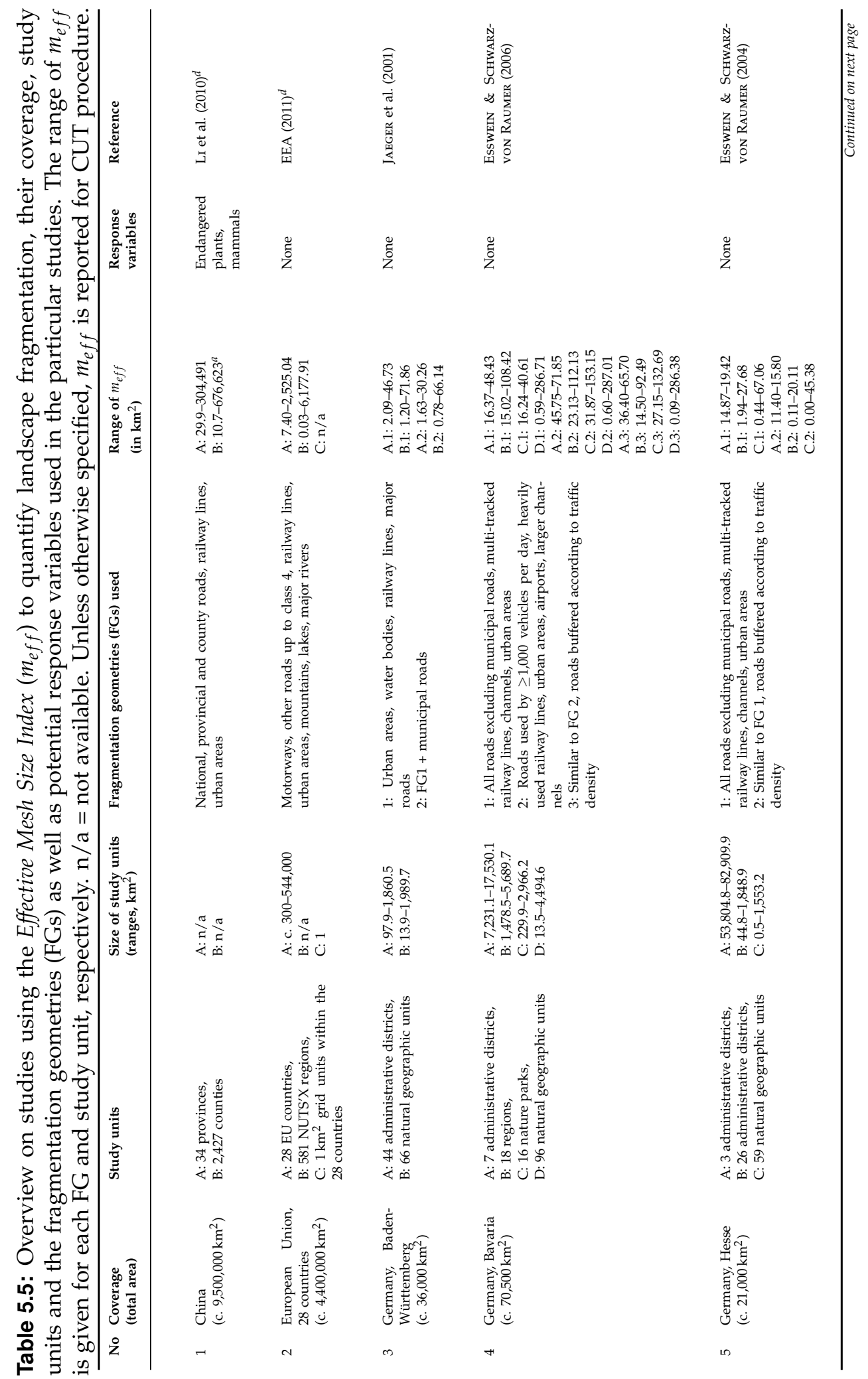




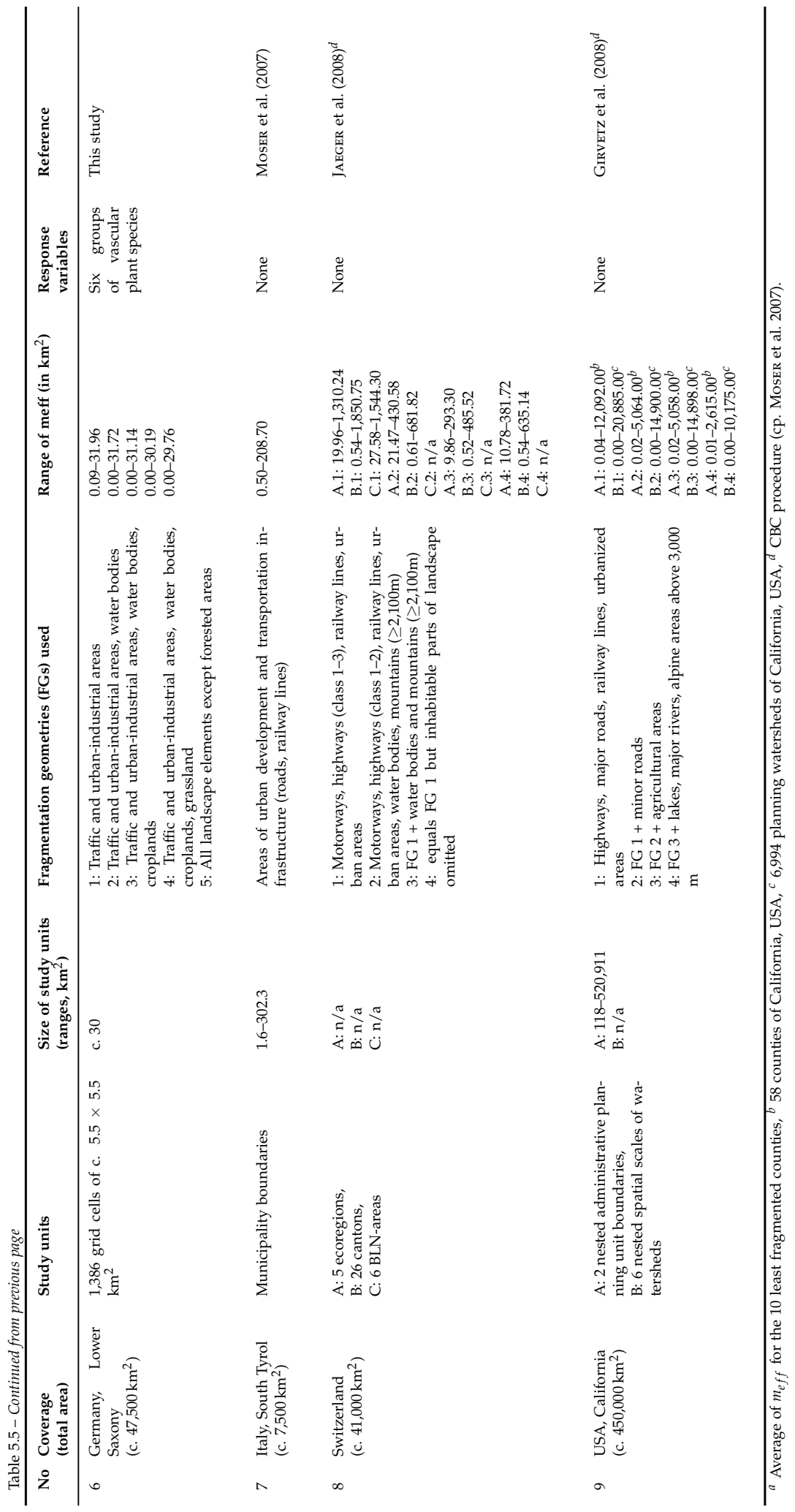




\section{Differentiating the effect of $\boldsymbol{m}_{\text {eff }}$ on species richness for selected plant groups}

The explanatory power of $m_{\text {eff }}$ compared to that of abiotic and spatial variables differed considerably among species groups. Surprisingly, species richness and landscape fragmentation were positively correlated for all species groups, with the exception of threatened species. Thus, species richness was highest in highly fragmented landscapes and was lowest in little fragmented landscapes.

For richness patterns of neophytes $m_{e f f}$ was an exceptionally important factor. That is not surprising, because alien species are known to be linked to urban habitats and linear landscape elements and are associated with human activities (e.g. PYšEK, 1998; Roy et al., 1999; Deutschewitz et al., 2003; Honnay et al., 2003; KüHN et al., 2003; LIPPE \& KowARIK, 2008). Archaeophytes, in contrast, are frequently found in rural environments (PYŠEK, 1998; DeutschewITZ et al., 2003), particularly in loess landscapes (KüHN et al., 2003) with a long colonization history (KüsteR, 1999). Today, most of these areas in our study area are intensively used and highly fragmented agricultural and/or industrial landscapes. The relation of archaeophyte richness with loess landscapes and major rivers in Lower Saxony was confirmed by SCHMIEDEL et al. (2011). As suggested by the high explanatory value of the environmental variables, areas of high archaeophyte richness are related to environmental conditions rather than to landscape fragmentation.

Only a very small proportion of native species richness was explained by $m_{\text {eff }}$, while environmental variables were more important. Even if weak, a negative relationship between $m_{e f f}$ and species richness was observed for this species group as well. This finding may be attributed to the fact that urban areas are commonly situated in naturally diverse ecoregions as shown by KüHN et al. (2004), who found that geological diversity is associated with areas of urbanisation in Germany. Furthermore, niche availability in urban areas is known to be rather high (DeUtschewitz et al., 2003; HonNAY et al., 2003; KüHN et al., 2004; WANIA et al., 2006; LIPPE \& KOWARIK, 2008). Possible adverse effects of fragmentation could therefore be masked.

Surprisingly, we found no effect of $m_{e f f}$ on threatened plant species richness patterns. Environmental conditions were better predictors of threatened plant species richness than was $m_{e f f}$. Similar to our results, Honnay et al. (2003), who studied the effect of different landscape indices as predictors for regional plant species richness, found the effect of landscape fragmentation on the richness of threatened species to be compensated by high landscape diversity. However, it seems that threatened species' occurrences are independent of the overall degree of landscape fragmentation (at least as long as it remains above a certain threshold). Threatened species are usually closely linked to ecologically valuable, often threatened habitat types. As discussed for Natura 2000 forest habitat types, characteristic forest species are limited in their dispersal ability and mostly linked to forests with long habitat continuity (CulmseE et al., 2014). At first, fragmentation may have only little effect on remnant 
populations as long as habitats do not vanish completely, yet the consequences of an extinction debt might be paid in the future (KuUsSAARI et al., 2009). However, the observed result may be an effect of the study scale being too coarse to reveal the relationship between landscape fragmentation and richness of threatened species (JACKSON \& FAHRIG, 2012). Further studies on different spatial scales are needed to provide evidence on the latter.

\section{Conclusions}

The assessment of landscape fragmentation and approaches for linking fragmented habitats by corridors are becoming increasingly important in nature conservation policy in Germany, as well as the rest of Europe (BFN, 2014; European Commission, 2011; BundesKabinetT, 2012). The EEA (2011, p. 58) even identified the analysis of the relationship between the levels of landscape fragmentation and biodiversity as "one of the most important areas for future research". In this context, measures of fragmentation are increasingly used to assess the status of a landscape (STRAND et al., 2007; BMU, 2010). The Effective Mesh Size $m_{e f f}$ is one of the most frequently applied indices in studies on landscape fragmentation. While many of these studies have pointed out the ecological relevance of fragmentation in general and $m_{e f f}$ in particular (JAEGER, 2000; JAEGER et al., 2008; GIRVETZ et al., 2008; LI et al., 2010), to our knowledge, until now no landscape-scale study has evaluated the impact of $m_{e f f}$ on species richness. By addressing this issue, our study has contributed to increasing the understanding of this relationship. We conclude that, as long as relevant fragmentation geometries for the landscapes under consideration are chosen, $m_{e f f}$ can act as a meaningful measure to explain vascular plant species richness patterns. Out of the five types of $m_{e f f}$ used in our study, two were found to be relevant for species richness, both having been used in previous studies (JAEGER et al., 2001; GIRVETZ et al., 2008; JAEGER et al., 2008). We further conclude that it is important to consider not only all vascular plant species, but also several smaller species groups. Species included in these groups should differ in their residence or threat status or in other characteristics in order to obtain more precise results on habitat requirements that facilitate interpretations.

Because fragmentation is a landscape-scale process, its relationship with biodiversity should accordingly be studies at this scale (McGARIGAL \& CUSHMAN, 2002; FAHRIG, 2003). However, when evaluating 100 and 135 fragmentation studies, respectively, McGarigal \& Cushman (2002) and FAHrig (2003) recognised that about $50 \%$ of all studies were actually conducted at the patch scale. This is obviously related to the practical difficulties arising from large-scale experiments. Thus, to be able to study the effects of landscape fragmentation on biodiversity, it is important to develop new methods for their assessment. Our approach avoids such difficulties by using readily available high-resolution data on land cover and the distribution of vas- 
cular plant species. As such data are becoming increasingly available in more and more countries (CULMSEE et al., 2014) and because computational power is rapidly increasing, our approach is easily extendable to other - even larger - study areas.

Further studies are needed to improve our understanding of the relationship of species richness and landscape fragmentation. The transferability of our results to other spatial scales should be tested, as well as the applicability of the method to less human-dominated landscapes. Presumably, effects of landscape fragmentation on species in highly transformed Central European landscapes differ from those in more natural landscapes. Thus, further studies are encouraged to bridge the gap between the assessment of landscape fragmentation, which is methodologically already advanced, and its relevance to species diversity, which has been rarely tested in the past.

\section{Acknowledgements}

Funding of the project "Identification of indicator species groups of grassland and forest habitats for biodiversity monitoring and evaluation" by the German Federal Foundation for the Environment, grant number DBU 26752, is gratefully acknowledged. We would like to thank all volunteers who reported vascular plant species occurrences in Lower Saxony, and the Lower Saxony Water Management, Coastal Defence and Nature Conservation Agency (NLWKN) for providing these data. We thank Laura Sutcliffe for linguistic revision. Petr Šmilauer (University of South Bohemia, Ceske Budejovice, Czech Republic) helpfully advised us how to use PCNM statistics in CANOCO. 


\section{References}

BEHRE, K.-E. (2008): Landschaftsgeschichte Norddeutschlands: Umwelt und Siedlung von der Steinzeit bis zur Gegenwart. Wachholtz, Neumünster. 300 pp.

Beyer, H. L. (2004): Hawth's Analysis Tools for ArcGIS. URL http://www. spatialecology.com/index.php.

BFN (Bundesamt FÜr NATURschutz) (2014): Zerschneidung - Wiedervernetzung. URL http://www.bfn.de/0306_zerschneidung.html. Retrieved 03/16/2014.

BMU (BUndesministerium FÜr UMWelt, NATURschutz UND REAKTORSicherHEIT) (2010): Indikatorenbericht 2010 zur Nationalen Strategie zur biologischen Vielfalt. URL http://www.bfn.de/fileadmin/MDB/documents/themen/ monitoring/Indikatorenbericht-2010_NBS_Web.pdf. Retrieved 07/15/2013.

BMU (Federal Ministry for the Environment, Nature Conservation AND Nuclear SAFETY) (2007): National strategy on biological diversity. URL http://www.bmub.bund.de/fileadmin/bmu-import/files/english/ pdf/application/pdf/broschuere_biolog_vielfalt_strategie_en_bf.pdf. Retrieved 07/01/2013.

Bohn, U. \& NeuhäUsL, R. (2000/2003): Map of the Natural Vegetation of Europe. Scale 1:2,500,000: Part 1: Explanatory text with CD-ROM; part 2: Legend. Landwirtschaftsverlag, Münster.

Booy, G.; Hendriks, R. J. J.; Smulders, M. J. M.; Groenendael, J. M. v. \& Vosman, B. (2000): Genetic diversity and the survival of populations. Plant Biology 2: 379-395.

Borcard, D. \& Legendre, P. (2002): All-scale spatial analysis of ecological data by means of principal coordinates of neighbour matrices. Ecological Modelling 153: 5168.

Borcard, D.; Legendre, P.; Avois-Jacquet, C. \& Tuomisto, H. (2004): Dissecting the spatial structure of ecological data at multiple scales. Ecology 85: 1826-1832.

Botham, M. S.; Rothery, P.; Hulme, P. E.; Hill, M. O.; Preston, C. D. \& Roy, D. B. (2009): Do urban areas act as foci for the spread of alien plant species? An assessment of temporal trends in the UK. Diversity and Distributions 15: 338-345.

Bowen, G. W. \& Burgess, R. L. (1981): A quantitative analysis of forest island pattern in selected Ohio landscapes. ORNL/TM7759. Oak Ridge National Laboratory, Oak Ridge, Tennessee. 122 pp.

BRAAK, J. F. C. T. \& Šmilauer, P. (2002): Canoco reference manual and CanoDraw for Windows user's guide: Software for canonical community ordination (version 4.5). Microcomputer Power, Ithaca. 500 pp. 
BundeSKabinetT (2012): Bundesprogramm Wiedervernetzung: Beschlossen vom Bundeskabinett am 29. Februar 2012. URL http:/ / www.bmub.bund.de/fileadmin/ bmu-import/files/pdfs/allgemein/application/pdf/bundesprogramm_ wiedervernetzung_bf.pdf. Retrieved 03/16/2014.

Cousins, S. A. O. (2009): Extinction debt in fragmented grasslands: paid or not? Journal of Vegetation Science 20: 3-7.

Cousins, S. A. O.; OHLson, H. \& ErIKsson, O. (2007): Effects of historical and present fragmentation on plant species diversity in semi-natural grasslands in Swedish rural landscapes. Landscape Ecology 22: 723-730.

Culmsee, H. (2013): Biodiversität in der mitteleuropäischen Kulturlandschaft. In: Siegesmund, S. \& SNethlage, R. (eds.) Naturstein in der Kulturlandschaft, Mitteldeutscher Verlag, Halle (Saale). pp. 30-38.

Culmsee, H.; Schmidt, M.; Schmiedel, I.; Schacherer, A.; Meyer, P. \& Leuschner, C. (2014): Predicting the distribution of forest habitat types using indicator species to facilitate systematic conservation planning. Ecological Indicators 37: 131-144.

DARK, S. J. (2004): The biogeography of invasive alien plants in California: an application of GIS and spatial regression analysis. Diversity and Distributions 10: 1-9.

Debinski, D. M. \& Holt, R. D. (2000): A survey and overview of habitat fragmentation experiments. Conservation Biology 14: 342-355.

Deutschewitz, K.; Lausch, A.; KüHN, I. \& Klotz, S. (2003): Native and alien plant species richness in relation to spatial heterogeneity on a regional scale in Germany. Global Ecology and Biogeography 12: 299-311.

DWD (1961-1990): Gridded data sets of long-term area averages in Germany: Resolution $1 \mathrm{~km}^{2}$ : Updated 05 May 2009.

EEA (European Environment Agency) (2011): Landscape fragmentation in Europe: Joint EEA-FOEN report, EEA Report, vol. 2. URL http://www.eea.europa. $\mathrm{eu} /$ publications/landscape-fragmentation-in-europe. Retrieved 05/15/2013.

ESRI INC. (2002): ArcGIS-ArcView 3.3.

ESRI INC. (2007): ArcGis-ArcInfo 9.2.

Esswein, H. \& Schwarz-von Raumer, H.-G. (2004): Analyse der Landschaftszerschneidung in Hessen. Endbericht im Auftrag des Hessischen Landesamtes für Umwelt und Geologie. URL http://www.hlug.de/fileadmin/dokumente/ nachhaltigkeit/landzerschneidung.pdf. Retrieved 05/10/2013. 
Esswein, H. \& Schwarz-von Raumer, H.-G. (2006): Darstellung und Analyse der Landschaftszerschneidung in Bayern. Endbericht im Auftrag des Bayerischen Landesamtes für Umweltschutz. URL http://www.lfu.bayern.de/natur/ landschaftszerschneidung/unzerschnittene_raeume/doc/bericht_lz_bz_06.pdf.

European Commission (2011): Our life insurance, our natural capital: an EU biodiversity strategy to 2020: Communication from the commission to the European parliament, the council, the economic and social committee and the committee of the regions. URL http://eur-lex.europa.eu/legal-content/EN/TXT/?uri=CELEX: 52011DC0244. Retrieved 05/16/2013.

EwERs, R. M. \& Didham, R. K. (2006): Confounding factors in the detection of species responses to habitat fragmentation. Biological Reviews 81: 117-142.

FAHRIG, L. (2003): Effects of habitat fragmentation on biodiversity. Annual Review of Ecology, Evolution, and Systematics 34: 487-515.

FGSV (ForschungSgeSELlschaft FÜr StRASSEN- UND VerKeHRSWESEN) (1996): Richtlinien für die Anlage von Straßen, Teil: Querschnitte (RAS-Q 96).

Forman, R. T. T. (1995): Land mosaics: The ecology of landscapes and regions. Cambridge Univ. Press, Cambridge. 632 pp.

GARve, E. (2004): Rote Liste und Florenliste der Farn- und Blütenpflanzen in Niedersachsen und Bremen. Informationsdienst Naturschutz Niedersachsen 24: 1-76.

GARVE, E. (2007): Verbreitungsatlas der Farn- und Blütenpflanzen in Niedersachsen und Bremen. Naturschutz und Landschaftspflege in Niedersachsen 43: 1-507.

Gharadjedaghi, B.; Heimann, R.; Lenz, K.; Martin, C.; Pieper, V.; Schulz, A.; Vahabzadeh, A.; FincK, P. \& Riecken, U. (2004): Verbreitung und Gefährdung schutzwürdiger Landschaften in Deutschland. Natur und Landschaft 79: 71-81.

Girvetz, E. H.; Thorne, J. H.; Berry, A. M. \& Jaeger, J. A. G. (2008): Integration of landscape fragmentation analysis into regional planning: A statewide multi-scale case study from California, USA. Landscape and Urban Planning 86: 205-218.

HaILA, Y. (2002): A conceptual genealogy of fragmentation research: From island biogeography to landscape ecology. Ecological Applications 12: 321-334.

Helm, A.; Hanski, I. \& PäRtel, M. (2006): Slow response of plant species richness to habitat loss and fragmentation. Ecology Letters 9: 72-77.

Honnay, O.; Jacquemyn, H.; Bossuyt, B. \& Hermy, M. (2005): Forest fragmentation effects on patch occupancy and population viability of herbaceous plant species. New Phytologist 166: 723-736. 
Honnay, O.; Piessens, K.; Landuyt, W. v.; Hermy, M. \& GulincK, H. (2003): Satellite based land use and landscape complexity indices as predictors for regional plant species diversity. Landscape and Urban Planning 63: 241-250.

JACKSON, H. B. \& FAHRIG, L. (2012): What size is a biologically relevant landscape? Landscape Ecology 27: 929-941.

JAEger, J. A. G. (2000): Landscape division, splitting index, and effective mesh size: New measures of landscape fragmentation. Landscape Ecology 15: 115-130.

Jaeger, J. A. G.; Bertiller, R.; Schwick, C.; Müller, K.; Steinmeier, C.; Ewald, K. C. \& GHazoul, J. (2008): Implementing landscape fragmentation as an indicator in the Swiss Monitoring System of Sustainable Development (MONET). Journal of Environmental Management 88: 737-751.

Jaeger, J. A. G.; Esswein, H.; Schwarz-von Raumer, H.-G. \& Müller, M. (2001): Landschaftszerschneidung in Baden-Württemberg. Naturschutz und Landschaftsplanung 33: 1-13.

KLOTZ, S.; KüHN, I. \& DuRKA, W. (2002): BIOLFLOR - Eine Datenbank zu biologischökologischen Merkmalen der Gefäßpflanzen in Deutschland. Schriftenreihe für Vegetationskunde 38: 1-334. URL http://www.biolflor.de.

KolB, A. (2008): Habitat fragmentation reduces plant fitness by disturbing pollination and modifying response to herbivory. Biological Conservation 141: 2540-2549.

Krause, B. \& Culmsee, H. (2013): The significance of habitat continuity and current management on the compositional and functional diversity of grasslands in the uplands of Lower Saxony, Germany. Flora 208: 299-311.

Krause, B.; Culmsee, H.; Wesche, K.; Bergmeier, E. \& Leuschner, C. (2011): Habitat loss of floodplain meadows in north Germany since the 1950s. Biodiversity and Conservation 20: 2347-2364.

Krauss, J.; Bommarco, R.; Guardiola, M.; Heikkinen, R. K.; Helm, A.; Kuussaari, M.; Lindborg, R.; Ockinger, E.; Partel, M.; Pino, J.; Poyry, J.; RaAtikainen, K. M.; Sang, A.; Stefanescu, C.; Teder, T.; Zobel, M. \& Steffan-Dewenter, I. (2010): Habitat fragmentation causes immediate and time-delayed biodiversity loss at different trophic levels. Ecology Letters 13: 597-605.

KüHN, I.; BRANDL, R. \& KLOTZ, S. (2004): The flora of German cities is naturally species rich. Evolutionary Ecology Research 6: 749-764.

KühN, I.; Brandl, R.; MaY, R. \& KLOTZ, S. (2003): Plant distribution patterns in Germany - Will aliens match natives? Feddes Repertorium 114: 559-573. 
Küster, H. (1999): Geschichte der Landschaft in Mitteleuropa: Von der Eiszeit bis zur Gegenwart. Beck, München. 423 pp.

Kuussaari, M.; Bommarco, R.; Heikkinen, R. K.; Helm, A.; Krauss, J.; Lindborg, R.; Ockinger, E.; Partel, M.; Pino, J.; Roda, F.; Stefanescu, C.; Teder, T.; Zobel, M. \& StefFan-Dewenter, I. (2009): Extinction debt: a challenge for biodiversity conservation. Trends in Ecology \& Evolution 24: 564-571.

LBEG (Landesamt für Bergbau, Energie und Geologie) (2003): Digitale Bodenkundliche Übersichtskarte 1: 50000 (BÜK50).

Legendre, P. \& Legendre, L. (2012): Numerical ecology. 3 edn. Elsevier, Amsterdam and Boston. 990 pp.

Leuschner, C.; Wesche, K.; Meyer, S.; Krause, B.; Steffen, K.; Becker, T. \& CulmSEE, H. (2013): Veränderungen und Verarmung in der Offenlandvegetation Norddeutschlands seit den 1950er Jahren: Wiederholungsaufnahmen in Äckern, Grünland und Fließgewässern. Berichte der Reinhold-Tüxen-Gesellschaft 25: 166-182.

LGN (LANDESVERMESSUNG UND GEOBASISINFORMATION NiEdERSACHSEN) (2008): Amtliches Topographisch-Kartographisches InformationsSystem (ATKIS): Updated 17 December 2008.

Li, T.; Shilling, F.; Thorne, J. H.; Li, F.; Schott, H.; Boynton, R. \& Berry, A. M. (2010): Fragmentation of China's landscape by roads and urban areas. Landscape Ecology 25: 839-853.

LIPPE, M. v. D. \& KowARIK, I. (2008): Do cities export biodiversity? Traffic as dispersal vector across urban-rural gradients. Diversity and Distributions 14: 18-25.

McCune, B. \& KeON, D. (2002): Equations for potential annual direct incident radiation and heat load. Journal of Vegetation Science 13: 603-606.

McGarigal, K. (2014): Fragstats help. URL http://www.umass.edu/landeco/ research/fragstats/documents/fragstats.help.4.2.pdf. Retrieved 03/02/2014.

McGarigal, K. \& Cushman, S. A. (2002): Comparative evaluation of experimental approaches to the study of habitat fragmentation effects. Ecological Applications 12: 335-345.

McGarigal, K.; Cushman, S. A.; Neel, M. C. \& Ene, E. (2002): FRAGSTATS: Spatial pattern analysis program for categorical maps. Version 3. URL www.umass.edu/ landeco/research/fragstats/fragstats.html.

Moser, B.; Jaeger, J. A. G.; Tappeiner, U.; Tasser, E. \& Eiselt, B. (2007): Modification of the effective mesh size for measuring landscape fragmentation to solve the boundary problem. Landscape Ecology 22: 447-459. 
NLfB (Niedersächsisches LANDESAMt FÜr Bodenforschung) (1997): Böden in Niedersachsen, Teil 1: Bodeneigenschaften, Bodennutzung und Bodenschutz. URL http:/ /www.lbeg.de/extras/nlfbook/html/nds_main.htm. Retrieved 07/09/2010.

Nobis, M. P.; Jaeger, J. A. G. \& Zimmermann, N. E. (2009): Neophyte species richness at the landscape scale under urban sprawl and climate warming. Diversity and Distributions 15: 928-939.

Oksanen, J.; Blanchet, F. G.; Kindt, R.; Legendre, P.; Minchin, P. R.; O'Hara, R. B.; Simpson, G. L.; Sólymos, P.; Henry, M.; Stevens, H. \& Wagner, H. (2012): vegan: Community Ecology Package. URL http://CRAN.R-project.org/package=vegan.

Oostermeijer, J. G. B.; Vaneijck, M. W. \& Dennijs, J. C. M. (1994): Offspring fitness in relation to population size and genetic variation in the rare perennial plant species Gentiana pneumonanthe (Gentianaceae). Oecologia 97: 289-296.

PARKs, S. (2004): ArcScript Heat Load Index. URL http://arcscripts.esri.com/details. asp?dbid=13368. Retrieved 12/18/2010.

Poschlod, P.; Bonn, S.; Kiefern, S.; Fischer, S.; Fuchs, A.; JAckel, A.-K.; LilienTHAL, D. \& TRÄNKLE, U. (1997): Die Ausbreitung von Pflanzenarten und populationen in Raum und Zeit am Beispiel der Kalkmagerrasen Mitteleuropas. Berichte der Reinhold-Tüxen-Gesellschaft 9: 139-157.

PYšEK, P. (1998): Alien and native species in Central European urban floras: a quantitative comparison. Journal of Biogeography 25: 155-163.

R Development Core Team (2012): R: A language and environment for statistical computing. URL http://www.R-project.org.

Rodriguez-Loinaz, G.; AmezagA, I. \& OnAindia, M. (2012): Does forest fragmentation affect the same way all growth-forms? Journal of Environmental Management 94: $125-131$.

Roy, D. B.; Hill, M. O. \& Rothery, P. (1999): Effects of urban land cover on the local species pool in Britain. Ecography 22: 507-515.

Schmiedel, I.; Schacherer, A.; Hauck, M.; Schmidt, M. \& Culmsee, H. (2011): Verbreitungsmuster der Farn- und Blütenpflanzen in Niedersachsen und Bremen unter Berücksichtigung ihres Einbürgerungsstatus und ihrer Gefährdungssituation. Tuexenia 31: 211-226.

Stoate, C.; Boatman, N.; Borralho, R.; Carvalho, C. R.; Snoo, G. R. D. \& Eden, P. (2001): Ecological impacts of arable intensification in Europe. Journal of Environmental Management 63: 337-365. 
Strand, H.; Höft, R.; Strittholt, J.; Miles, L.; Horning, N.; Fosnight, E. \& Turner, W. (2007): Sourcebook on remote sensing and biodiversity indicators. 203 pp.

Tilman, D.; May, R. M.; Lehman, C. L. \& NowaK, M. A. (1994): Habitat destruction and the extinction debt. Nature 371: 65-66.

TscharntKe, T.; Klein, A. M.; Kruess, A.; Steffan-Dewenter, I. \& Thies, C. (2005): Landscape perspectives on agricultural intensification and biodiversity - ecosystem service management. Ecology Letters 8: 857-874.

Vos, W. \& Meekes, H. (1999): Trends in European cultural landscape development: perspectives for a sustainable future. Landscape and Urban Planning 46: 3-14.

Walz, U. (2005): Landschaftszerschneidung in Grenzräumen - Sachsen und die Sächsisch-Böhmische Schweiz. GAIA 14: 171-174.

WANIA, A.; KÜHN, I. \& KLOTZ, S. (2006): Plant richness patterns in agricultural and urban landscapes in Central Germany - spatial gradients of species richness. Landscape and Urban Planning 75: 97-110.

Young, A. G. \& Clarke, G. M. (2000): Genetics, demography and viability of fragmented populations. Cambridge University Press, Cambridge. 460 pp.

Zimbres, B.; Furtado, M. M.; Jácomo, A. T. A.; Silveira, L.; Sollmann, R.; TôrRes, N. M.; Machado, R. B. \& Marinho-Filho, J. (2013): The impact of habitat fragmentation on the ecology of xenarthrans (Mammalia) in the Brazilian Cerrado. Landscape Ecology 28: 259-269. 



\section{Plant species richness along a gra- dient of landscape modification}

Schmiedel, I.; Bergmeier, E. \& Culmsee, H.: Plant species richness along a gradient of landscape modification in Lower Saxony, Germany. Submitted to journal. 


\section{Abstract}

Several concepts of landscape modification, landscape naturalness and hemeroby have been put forward to quantify the extent of human activities and impact on landscapes. However, despite numerous studies on the human influence on landscapes, the relationship between these concepts and biodiversity has rarely been tested. On a grid of 1386 lat/long cells (landscape units, c. $30 \mathrm{~km}^{2}$ ) we used simple information on land cover and easily measurable landscape metrics to identify a gradient of landscape modification for the model region of Lower Saxony, Germany. We linked the gradient of landscape modification intensity to richness patterns of eight groups of vascular plants that were aggregated using residence and threat status and habitat preferences of the species.

K-means cluster analysis revealed a gradient of six landscape modification levels ranging from highly-fragmented urban landscapes to little-fragmented landscapes with large proportions of forest. Richness of all species groups differed significantly along the gradient. For the total and native species pools, for forest, low-nutrient indicating and threatened plants richness peaked at both ends of the modification intensity gradient. For neophytes, urban plants and high-nutrient indicators, richness decreased along the gradient from urbanised to less modified landscapes.

Due to its simplicity and coarse scale, our approach may easily be applied to other study areas without comprehensive data on land cover. Furthermore, following proper interpretation by experts, it can be used to identify and preselect priority landscapes for nature conservation planning.

\section{Keywords}

Landscape metrics; diversity; hemeroby; landscape naturalness; neophytes; urbanisation 


\section{Introduction}

Over recent decades, tremendous man-induced changes in land cover, increasing landscape fragmentation, degradation and intensification have occurred in landscapes all over Europe (Bastian \& Bernhardt, 1993; Meeus, 1995; Stoate et al., 2001; MANDER et al., 2004; Foley et al., 2005; WESCHE et al., 2012). Such landscape modifications have already led to large losses in (semi-)natural vegetation, biodiversity and ecosystem functions (VEAC, 2010) and are expected to cause even more losses in the future (SALA et al., 2000). Researchers have developed ideas on 'landscape modification' (Forman \& Godron, 1986) and the related concepts of 'landscape naturalness' (MACHAdo, 2004; Litra \& SePp, 2009; Winter et al., 2010), 'hemeroby' (Sukopp, 1976; KowAriK, 1988; GrabHerr et al., 1998; STEINHARDt et al., 1999; WrbKa et al., 2004; Walz \& Stein, 2014), 'urbanity' (Hill et al., 2002; WrbKa et al., 2004) and 'degree of culturalness' (JANSEN et al., 2009) that are used to quantify the degree of modification a landscape has experienced. For plant species, qualifying indices are available that are based on the concepts of hemeroby (i. e., estimating the degree of human influence on the plant's environment) or urbanity (i.e., strength of association with urban environments) (Jalas, 1955; KLOTZ \& KüHN, 2002). Despite the vast number of studies exploring concepts of landscape modification or investigating human influences on biodiversity, the relationship between the two has rarely been tested directly at large scales (but see NABE-NiELSEN et al., 2010 for animal populations). Systematic studies on biodiversity patterns in modified landscapes are, however, urgently needed to assess overall effects of human impact on biodiversity and to support landscape-scale conservation planning (DAILY, 2001; FISCHER \& LINDENMAYER, 2007).

There are numerous approaches to quantify the magnitude of deviation from the natural state of a landscape. While some authors have based their assessment of the landscape status solely on land cover data (STEINHARDT et al., 1999), others used multiple parameters to describe the degree of hemeroby (GRABHERR et al., 1998; WINTER et al., 2010; WALz \& STEIN, 2014) or naturalness (LIIRA \& SEPP, 2009; WINTER et al., 2010) of a landscape. Most of these studies were conducted for forest habitats, but WALz \& STEIN (2014) developed an indicator of hemeroby applicable to the whole of Germany. The authors used high-resolution data on land cover and spatial information on the potential natural vegetation to derive the degree of hemeroby. They assigned the land cover categories to seven levels of hemeroby and calculated landscape hemeroby for various reference units.

Most studies on the hemeroby, naturalness or modification of a landscape or biotope are dependent on extensive field data and/or other detailed information. For many countries, such comprehensive data are not available. To avoid this problem, land cover data (either directly taken from existing digital landscape models or derived from remote sensing approaches) might prove suitable for the assessment of landscape modification and its relation to biodiversity. STEINHARdT et al. (1999, p. 2) considered data on land use/land cover as the "interface between the natural 
conditions of the landscape and the influence of man". These data are "attainable within a reasonable time and with reasonable effort" (as demanded by MACHADO, 2004, p. 100). Various landscape metrics based on land cover data have been used to describe and quantify changes in landscape patterns and the composition and configuration of landscapes (LUCK \& WU, 2002; YU \& NG, 2006; Olsen et al., 2007). Landscape parameters found to decrease when landscape modification increases are the amount of (semi-)natural land cover types (henceforth LCTs), as well as the average sizes of habitat patches (Forman \& Godron, 1986; Miller et al., 1997; Moser et al., 2002). As patch sizes decrease, habitats are subdivided into smaller fragments, patch numbers increase and core area sizes decrease. These processes may reduce the connectivity of patches, increase isolation between suitable habitats and enhance edge effects (Harper et al., 2005; Mascarua Lopez et al., 2006). Depending on the quality of the LCT under consideration, such landscape changes can either be interpreted as increasing the structural diversity of the landscape or increasing its subdivision (LANG \& BLASCHKE, 2007).

Several studies have investigated the relationship between some of the above mentioned parameters and biota. Kerr \& Deguise (2004) and Fischer \& LindenMayer (2007) reported that native species benefit from the presence of natural vegetation and suggested that a decrease in the amount and size of natural habitat patches (i. e., an increase in the fragmentation of the habitat) provokes a decrease in native species richness. In contrast, many alien species are associated with human activities and commonly linked to urban habitats and to linear landscape elements. They may thus benefit from the modification of the landscape (e. g. Deutschewitz et al., 2003; HonNAY et al., 2003; KüHN et al., 2003). Generally, high habitat heterogeneity was found to be linked to large numbers of both native and alien species (DEUTSCHEwITz et al., 2003).

As Forman \& GodRon (1986, p. 286) pointed out, it is "neither possible nor useful" to separately consider the various types of human influence when evaluating the status of a landscape. Instead, the combined effects of all human influences visible at the landscape scale should be taken into account. In doing so, landscapes can be arranged along a 'landscape modification gradient'. In their well-known text on landscape ecology, Forman \& Godron (1986) proposed a 5-level concept on landscape modification ranging from natural to urban landscapes. Inspired by this idea, our aim was to analogously identify a gradient of landscape modification intensity based on simple information on land cover and easily measurable landscape metrics. We aimed to link the modification gradient to richness patterns of vascular plant species that were available as presence data on latitude/longitude grid cells covering the complete study area. We used LCTs that captured large proportions of the landscape and were easy to identify using remote sensing approaches. In contrast to WALz \& STEIN (2014), our analysis does not make any a priori statements about the naturalness or hemeroby of the LCTs under consideration. In the analysis, we 
used the total number of vascular plant species reported for the study area as well as seven sub-groups of species aggregated using their residence and threat status as well as information on habitat preferences.

We hypothesised:

- Based on landscape metrics used to assess the composition and configuration of a landscape, clusters of landscapes with similar characteristics can be differentiated. These can be placed along a gradient of landscape modification, as proposed by Forman \& GODRON (1986), spanning from (semi-)natural (mostly forested) to urbanised landscapes.

- The landscape clusters are meaningful for vascular plant species richness, i.e., species numbers vary significantly along the gradient of landscape modification intensity.

- Species numbers of different vascular plant species groups show different patterns along the gradient of landscape modification intensity.

\section{Material \& Methods}

\section{Study area}

The study was carried out in the German federal state of Lower Saxony (c. 47,500 km², Fig. 6.1a). As landscape formation history and natural environmental configuration, colonisation history and current land use vary widely across the state, Lower Saxony harbours a large number of different habitat types.

The coastal area in the north of Lower Saxony was formed in the Holocene. It comprises the Wadden Sea, its islands and salt marshes, the estuaries of the three largest rivers of Lower Saxony (Ems, Weser and Elbe) as well as impoldered former tidal wetlands. The latter are today mainly covered by grasslands, croplands and urban areas (DRACHENFELS, 2010).

The lowlands were formed during the Pleistocene and harbour various types of landscapes, ranging from intensively managed agricultural landscapes in the west to wooded landscapes in the east.

The uplands in the south have Mesozoic and Palaeozoic bedrock and display high landscape heterogeneity. The landscape of the less elevated parts of this area is characterised by a mosaic of croplands, grasslands and various forests, while the higher elevations in the southeast (Harz Mountains) are largely covered by forests.

The largest cities of Lower Saxony are Hanover and Braunschweig, both situated at the border of the lowland and upland areas, followed by Oldenburg in the northern lowlands and Osnabrück in the western uplands.

From the coast in the north to the Harz Mountains in the southeast, elevation ranges from 0-971 $\mathrm{m}$ a.s.l.. From the northwest to the east, the climate gradually changes 
from oceanic (mean annual precipitation up to $900 \mathrm{~mm}$ ) to suboceanic-subcontinental (c. $550 \mathrm{~mm}$; NLfB, 1997).

Lower Saxony was selected as model region because of its high landscape diversity and because the area is covered by a completed mapping program on the distribution of vascular plants with a resolution of c. $30 \mathrm{~km}^{2}$ (GARVE, 2007).

\section{Landscape composition and configuration}

Following the grid system of the topographical maps, the study area was subdivided into a regular grid of 1739 cells sized c. $5.5 \times 5.5 \mathrm{~km}^{2}\left(30 \mathrm{~km}^{2}\right.$; Fig. $\left.6.1 \mathrm{a}\right)$. The 353 grid cells situated at the border of the state that only partially covered the study area and a further three grid cells completely covered by water bodies were omitted. Thus, a total of 1383 grid cells remained in the analysis; in the following each of these will be treated as a landscape unit.

Landscape composition and configuration were derived from a digital landscape model (DLM; LGN, 2008) of Lower Saxony. The DLM vector data were categorised in eight LCTs (traffic, urban and industrial areas, water bodies, forests, grasslands, croplands, and an additional class that summarizes all remaining landscape elements) and then transformed to raster format with a resolution of $25 \mathrm{~m}^{2}$. Thirty different landscape metrics (Table 6) were calculated for each of the 1383 landscape units using Fragstats 3.3 (McGARIGAL et al., 2002). Ten of these indices describe landscape composition; the remaining 20 indices depict different aspects of landscape configuration.

We calculated the proportion of each LCT per landscape unit (Percentage of Landscape, PLAND). The diversity at the landscape level was assessed using the Shannon Diversity Index (SHDI). The latter takes into account all LCTs present in the landscape as well as their extent and is especially sensitive to the presence of rare LCTs (McGarigal et al., 2002).

The Effective Mesh Size Index (MESH) was used as an indicator to quantify the degree of fragmentation of a landscape unit. For its calculation, landscape elements that can act as barriers in the landscape (fragmentation geometries) have to be designated. In a previous analysis (SCHMIEDEL \& CUlMSEE, submitted), urban, industrial and traffic areas as well as water bodies were identified as suitable fragmentation geometries for the prediction of plant species richness. Thus, these LCTs were also used in the present study.

To capture average patch sizes, the Area-weighted Mean Patch Size Index (AREA_AM) was assessed. Together with the Number of Patches (NP), it supports assumptions about the fragmentation of a LCT and thus adds information to the general trend that can be derived from MESH. Patch complexity was evaluated using the Areaweighted Mean Shape Index (SHAPE_AM). Both, AREA_AM as well as SHAPE_AM were calculated on the landscape scale (i.e., averaged over all LCTs) as well as on the LCT level for forest, grassland and cropland areas, respectively; the number of patches was calculated for forest, grassland and cropland areas only. 


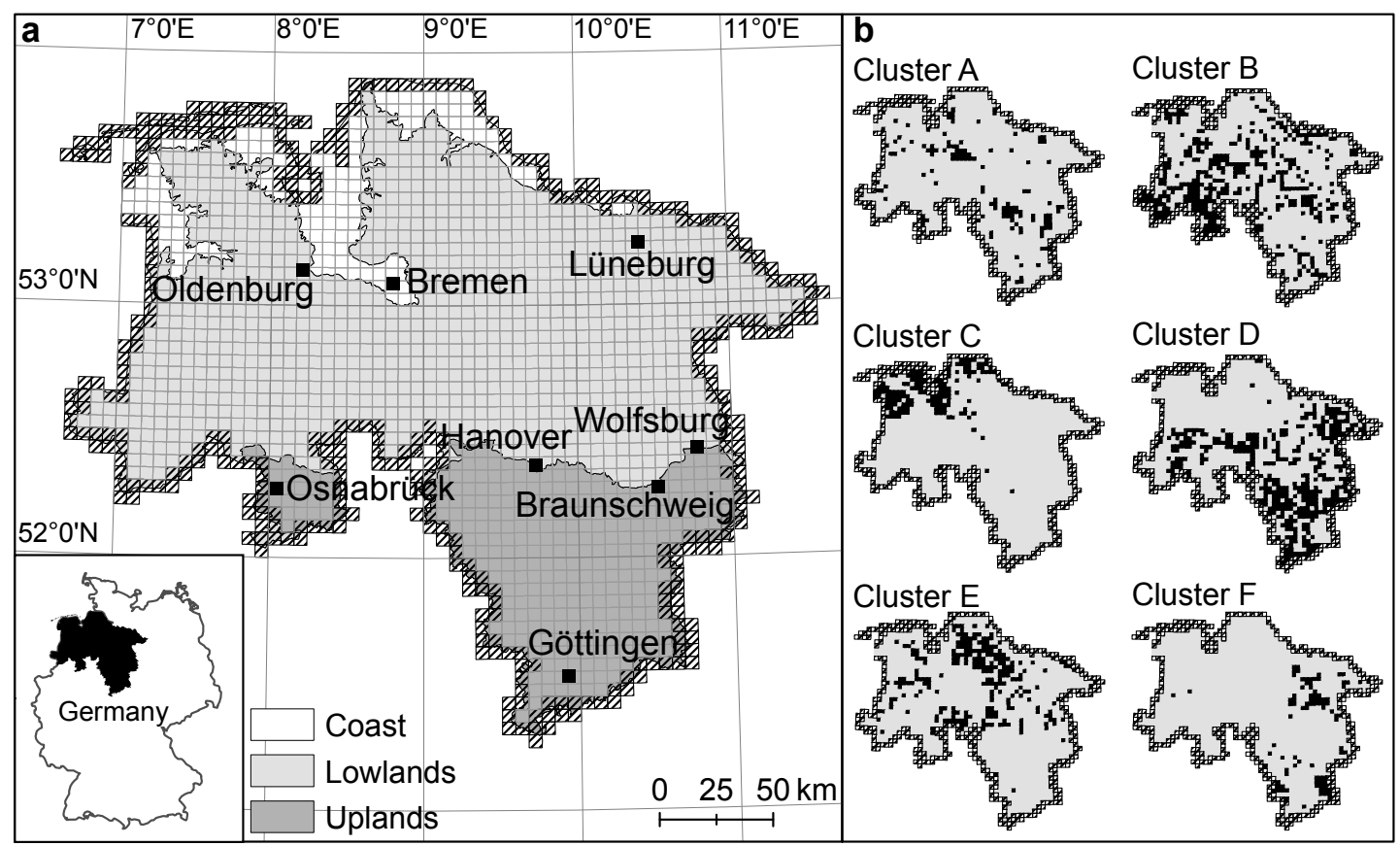

Figure 6.1: (a) The state of Lower Saxony, Germany, with its three major landscape components: coast, lowlands and uplands. The 1383 landscape units (size c. $5.5 \times 5.5 \mathrm{~km}^{2}$ or $30 \mathrm{~km}^{2}$ ) display the basic mapping units of the vascular plant survey program and represent the landscape units used in this analysis. Hatched grid cells were not considered in the analysis. (b) Distribution of landscape units included in clusters A to F in Lower Saxony. Maps with Transverse Mercator projection, Germany Zone 3 ("Deutsches Hauptdreiecksnetz").

Edge length was assessed using the Total Edge Index (TE) that sums up all edges a particular LCT shares with other LCTs.

As a measure for the isolation or rather connectivity of the patches of a LCT the Mean Proximity Index (PROX_MN) was assessed. It is calculated by considering size and distance of all neighbouring patches of the same type; the distance within patches that should be regarded as 'connected' has to be specified by the user ('search radius'; i.e., $100 \mathrm{~m}$ in the present analysis). It should be noted that only landscape connectivity and not habitat and ecological connectivity was assessed (cp. FIsCHER \& LINDENMAYER, 2007). TE and PROX_MN were calculated on the LCT level for forest, grassland and cropland areas, respectively.

For the LCT 'forest' the Total Core Area Index (TCA) and the Mean Core Area Index (CORE_MN) were assessed. The former measures the total amount of undisturbed core habitat after a user-specified edge buffer (i. e., $100 \mathrm{~m}$ in the present analysis) is eliminated (McGARIGAL et al., 2002). Analogously, CORE_MN quantifies the average amount of undisturbed core habitat in the landscape. 


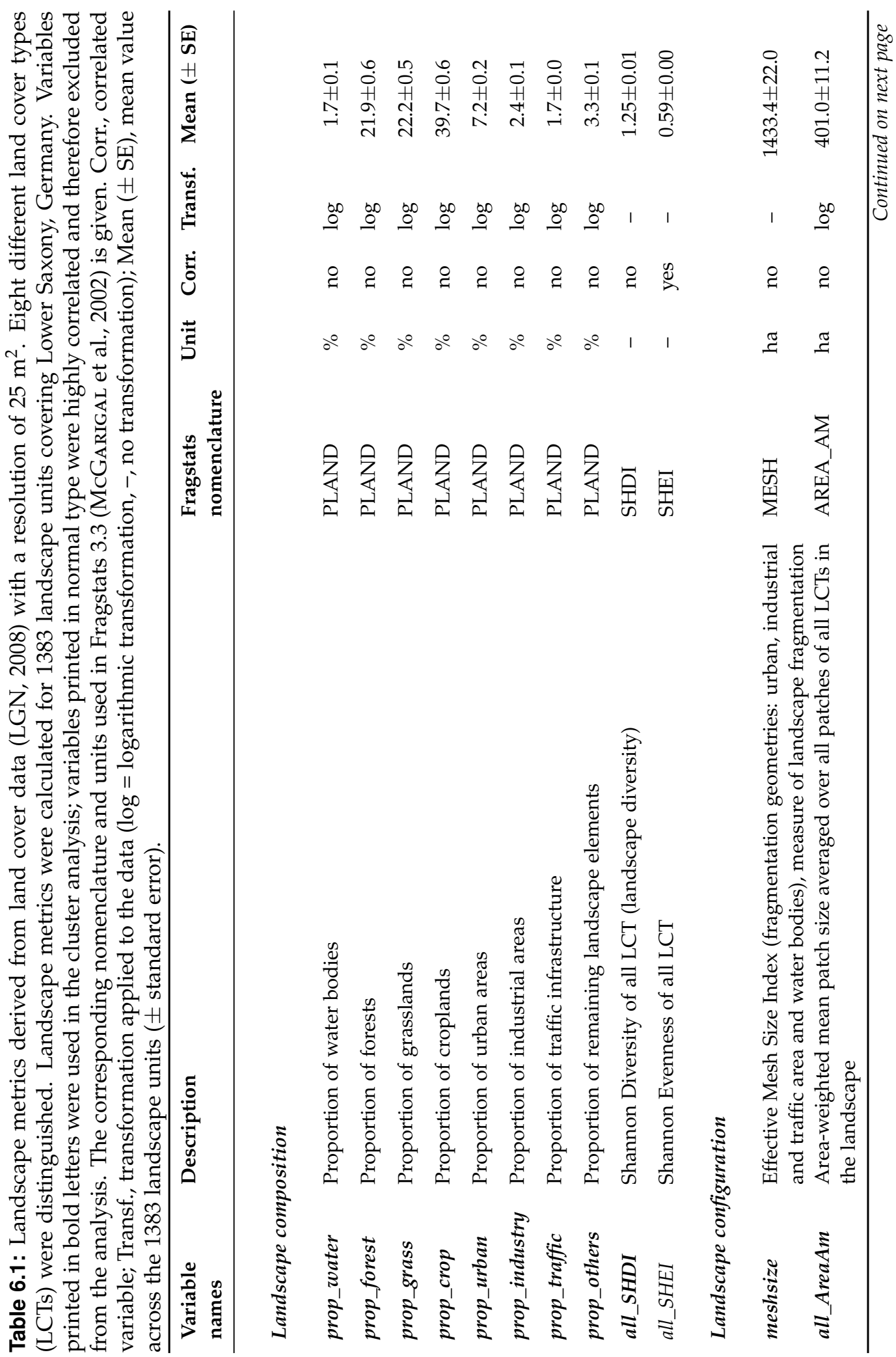




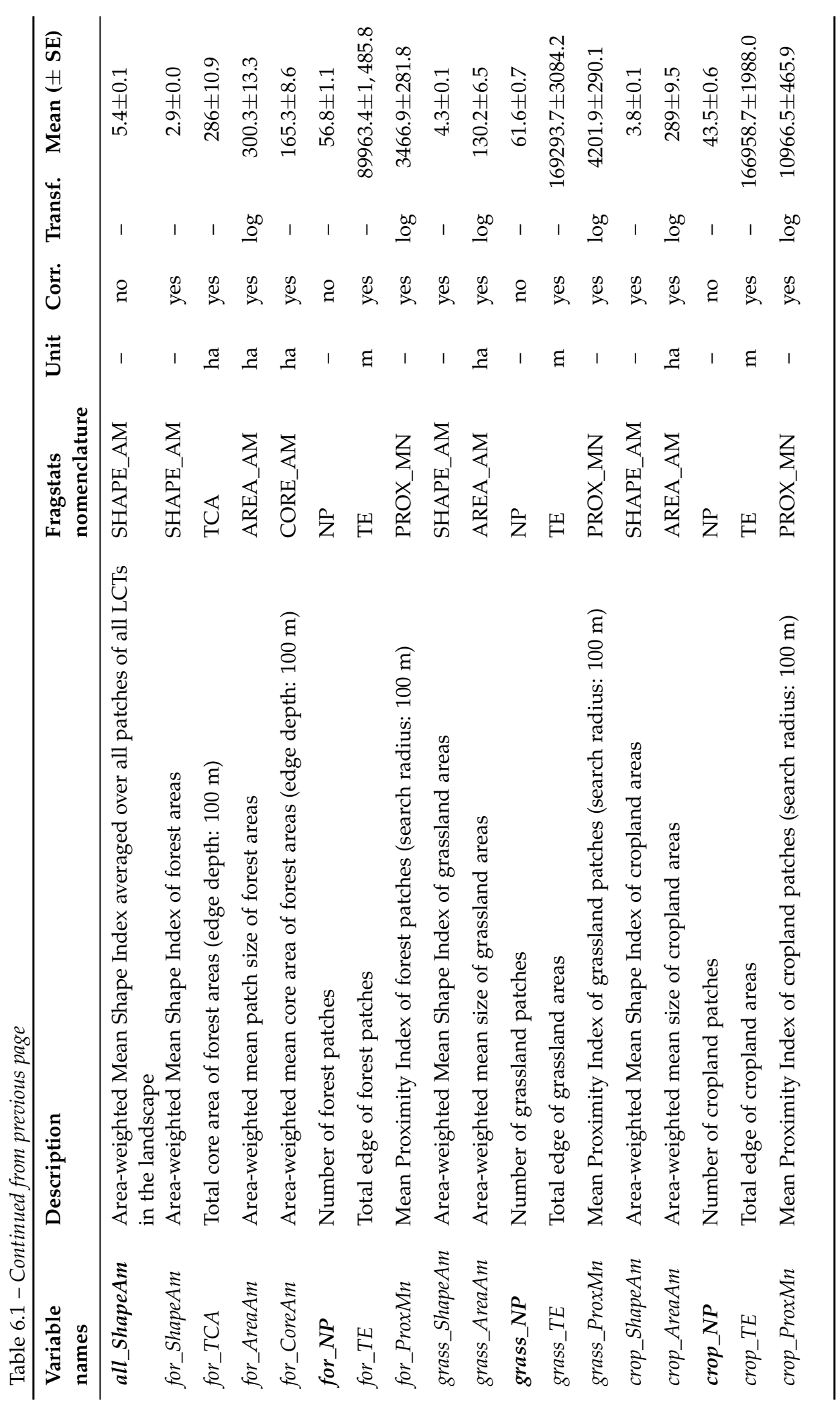


To improve statistical normality, the proportions of LCTs as well as the values obtained for AREA_AM and PROX_MN were log-transformed prior to the analysis. All landscape metrics were standardised to z-scores, because the range of values of the different indices strongly differed.

Landscape metrics were tested for correlation amongst each other using Spearman's rank correlation coefficient $\rho$. Highly correlated variables (Spearman's $\rho>$ $|0.7|$; i. e., 15 variables, see Table 6) were excluded from the further analysis, whereas the simple landscape composition metrics were preferred over the more complex indices of landscape configuration. In total, 15 landscape metrics remained in the analysis (Table 6).

\section{Cluster analysis}

Based on the 15 standardised landscape metrics, k-means clustering was applied to pool those landscape units with a similar landscape composition and configuration. The k-means analysis groups entities according to the similarity of their attributes. It was performed using the 'kmeansruns' function from 'fpc' package (HenNING, 2014) in $R$ version 2.15.2 ( $R$ Development Core Team, 2012). The function initialises the $\mathrm{k}$-means algorithm several times using different starting points. The number of runs as well as the number of iterations was set to 100. The function estimates the optimal number of clusters by considering the average silhouette width criterion. The latter provides a measure of how well each landscape unit lies within the cluster it was assigned to. The index ranges between +1 (well clustered) and -1 (poorly clustered) (Brock et al., 2008). The number of clusters to be compared was set to a minimum of 5 and a maximum of 20 clusters.

To determine the main gradient underlying the clusters, a PCA on the standardised variables was performed in $\mathrm{R}$ (function 'prcomp' in the 'stats' package). Clusters were sorted by the variable with most influence on the data set (i.e., prop_urban).

\section{Species numbers per cluster}

Plant species records were obtained from the data base of the vascular plant survey program of Lower Saxony (NLWKN 1982-2003) that holds information about the presence/absence of all vascular plant species occurring in the aforementioned $30 \mathrm{~km}^{2}$ landscape units (GARVE, 2007).

In the analysis, we used the total number of 1594 vascular plant species reported for the study area as well as seven sub-groups of species aggregated according to their residence status, their threat status and their association with particular habitats (Table 6.2). Threatened species were identified using the Red List of Lower Saxony (GARVE, 2004). Native species were distinguished from established neophytes (KLOTZ et al., 2002). Forest species (SснмIDT et al., 2011) were selected because they are habitat specialists that usually do not occur outside of forests. Among the eight LCTs 
Table 6.2: Total number of vascular plant species of Lower Saxony, Germany, and the number of species of seven sub-groups distinguished by residence status, threat status and habitat preferences. Species groups were overlapping to some extent (cp. Table A.8 in Appendix A).

\begin{tabular}{|c|c|c|}
\hline No. Species group & Definition & $\begin{array}{l}\text { Numbers } \\
\text { of species }\end{array}$ \\
\hline
\end{tabular}

Total vascular plant flora

$1 \quad$ All species

Residence status

2 Native species

3 Established neophytes
Total number of plant species recorded for Lower Saxony ${ }^{a, b}$

1594

Species native to Lower Saxony ${ }^{b, c}$

Species not native to Lower Saxony, established 16th century or later ${ }^{b, c}$

\section{Threat status}

4 Threatened species

Red-listed species (threat status 1, 2, 3, G, R) ${ }^{d}$

Habitat preferences

5 Forest species

6 Urban species

7 EIV species N 1-3

8 EIV species N 7-9
Species linked to forest habitats ${ }^{e}$ Species linked to urban habitats $f$ Species of nutrient-poor sites ${ }^{g} \quad 474$ Species of nutrient-rich sites ${ }^{h} \quad 306$

${ }^{a}$ NLWKN (1982-2003); ${ }^{b}$ GARVE (2007); ${ }^{c}$ KLotz et al. (2002); ${ }^{d}$ GARVE (2004); ${ }^{e}$ Categories 1.1 and 1.2, SCHMIDT et al. (2011); $f$ "Urbanity", categories 4 and 5, KLOTZ et al. (2002); 8 Ellenberg indicator value for nutrients (N) 1-3, BFN (2013); ${ }^{h}$ Ellenberg indicator value for nutrients (N) 7-9, BFN (2013).

of our analysis, we considered forests representing areas least influenced by humans. Following KLOTz et al. (2002), a group of urban species linked to settlements was defined (urbanity index 4 and 5). As eutrophication is one of the most important threats for temperate European ecosystems (DISE, 2011), two further species groups were assembled based on their association with nutrient-rich (Ellenberg Indicator Values, EIV N 7-9) and nutrient-poor habitats (EIV N 1-3), respectively (BFN, 2013). Species groups were overlapping to some extent (see Table A.8 in Appendix A). Subspecies were pooled to the corresponding species and species to the relevant aggregate of species if these were present in the data base.

Mean numbers of the total number of species reported for Lower Saxony and of the seven sub-groups were calculated per cluster. To test for significant differences between species numbers of the different clusters, a non-parametric Kruskal-Wallis test was performed in R. Multiple comparisons were made using the 'pgirmess' package (Giraudoux, 2013) at a 5\% significance level. 


\section{Results}

\section{Clusters of landscape modification intensity}

K-means clustering of the 1383 landscape units resulted in six clusters of varying size. The smallest cluster (F) comprised 90 and the largest cluster (D) 389 landscape units (Table 6.3). The average silhouette width value of 0.16 obtained for our six clusters indicated a higher similarity within clusters than between neighbouring clusters. Although this value was relatively low, the clusters were nevertheless well interpretable.

The results of PCA (Table 6.4) suggested that the most important landscape metric was the proportion of urban areas (prop_urban). Further variables highly correlated with PCA axis 1 were the Effective Mesh Size Index (meshsize) and the Area-Weighted Mean Patch Size Index averaged over all patches of all LCTs in the landscape units (all_AreaAm).

The six clusters showed the following features (see Table 6.3):

Cluster A contained only 131 landscape units (9\% of the total number of investigated landscape units) and was characterised by an extremely high landscape fragmentation (very low meshsize, low area-weighted mean patch size). Compared to the other clusters, the patches in this cluster showed very high landscape diversity (all_SHDI). Most parts of these landscapes were made up by urban-industrial and traffic areas, thus the landscape units included mainly large cities (Fig. 6.1b).

Cluster B, the second largest cluster in the analysis (28\% of the landscapes units), was characterised by medium to high landscape fragmentation (low meshsize, low area-weighted mean patch size) and medium to high landscape diversity. All LCTs occurred at medium proportions. Forests, grasslands and croplands were each represented by a large number of patches suggesting relatively small but numerous patches (i.e., highly fragmented). Landscape units of this cluster were dispersed all over Lower Saxony (Fig. 6.1b).

Cluster C, representing $11 \%$ of the landscape units, was characterised by numerous grassland patches, very large proportions of grassland and water bodies and low to very low proportions of forest and cropland. Landscape diversity as well as landscape fragmentation occurred at medium magnitude. The complexity of patches in the landscape was very high. Landscape units of this cluster were - with only few exceptions - mainly located in coastal and subcoastal areas and in the north-western lowlands (Fig. 6.1b).

Cluster D, the largest cluster in the analysis (28\% of the landscapes units), was dominated by croplands that on average covered more than $55 \%$ of the landscape. Forests occurred at medium proportions, while the proportions of urban-industrial and traffic areas as well as grasslands were small. The degree of fragmentation and landscape diversity were generally medium to low. Landscape units of this cluster were mainly distributed in the uplands and the eastern and central lowlands (Fig. 6.1b). 


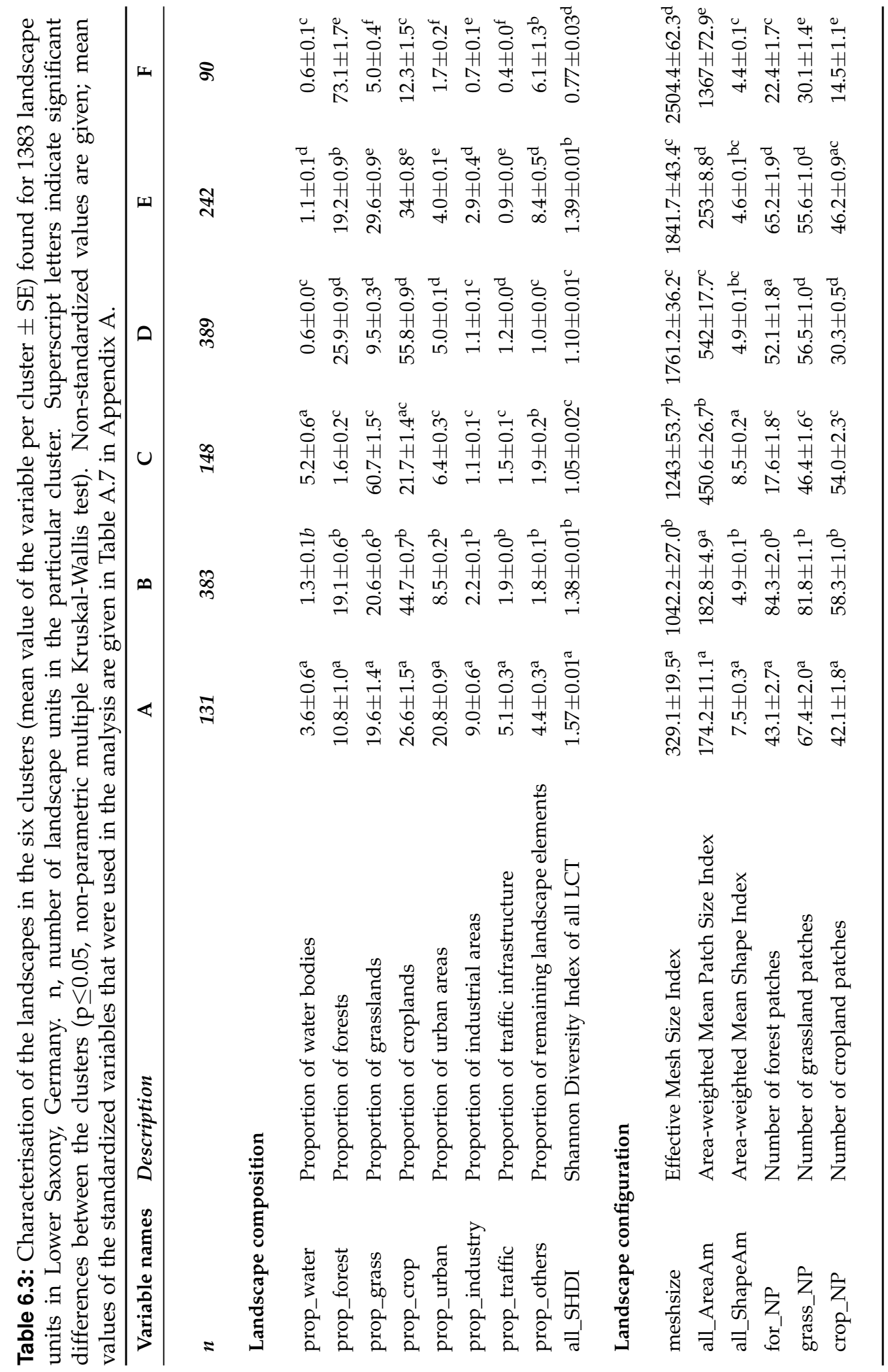


Cluster E, including $17 \%$ of the landscape units, was characterised by a mosaic of croplands (c. $35 \%$ ), grasslands (c. 30\%), forests (c. 20\%) and landscape elements subsumed under "others" (c. 9\%). Urban-industrial and traffic areas were present at small proportions. The degree of fragmentation and landscape diversity were generally medium to low. Landscape units of this cluster were - with one exception - all located in the lowlands (Fig. 6.1b).

Cluster $\mathbf{F}$, the smallest cluster in the analysis, containing 90 grid cells ( $7 \%$ of the landscapes units), was characterised by large proportions of forests and small proportion of all other LCTs. The values obtained for the Effective Mesh Size Index and area-weighted mean patch sizes were very high, thus the landscape fragmentation was low. Landscape diversity was very low. The number of grassland and cropland but also of forest patches was small. The latter in combination with the large proportion of forests suggested the occurrence of large coherent forests. These had a highly complex shape. The landscape units of this cluster were mainly situated in the Harz and Solling Mountains in the southeast of Lower Saxony as well as in the Luneburg Heath, which is part of the lowlands (Fig. 6.1b).

\section{Species richness patterns along the landscape modification gradient}

In all species groups, species richness differed significantly between the clusters (Fig. 6.2, Table A.9 in Appendix A). Richness of the total number of species reported for Lower Saxony decreased from the strongly modified landscape of cluster A to the grassland-dominated landscape of cluster $C$ and then increased to clusters $D, E$ and F. For the latter clusters, species numbers were highest in the cropland-dominated cluster D, followed by cluster $\mathrm{F}$ that was characterised by large proportions of forests (Fig. 6.2).

Corresponding patterns could be observed for the species groups of native, redlisted, forest and EIV species N1-3. While native and forest species followed the pattern of all species, the trend observed for Red List species and those species linked to nutrient-poor habitats showed a 'V shape' with the highest species numbers reported for clusters A (strongly modified landscape) and F (forest-dominated landscape) and the lowest for cluster C (grassland-dominated landscape).

The peak of species numbers in the strongly modified landscape of cluster A was repeated by the remaining species groups (neophytes, urban and EIV species N79), but, in contrast to the other groups, species richness more or less gradually decreased along the cluster gradient. This downward trend was only disrupted by the cropland-dominated cluster D that showed slightly higher species numbers than the surrounding clusters (Fig. 6.2). 
a) All species

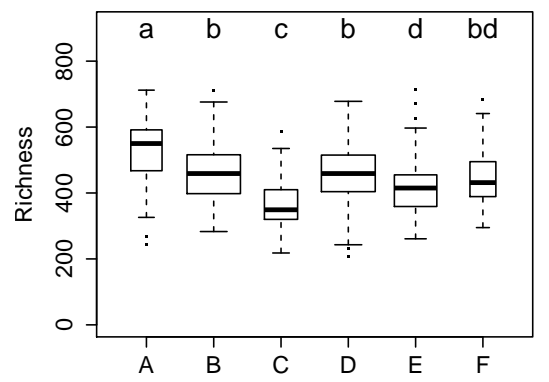

c) Established neophytes

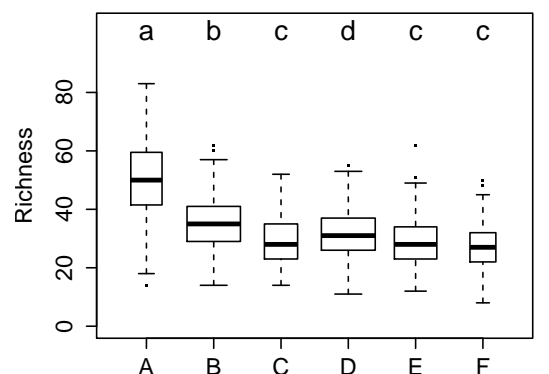

e) Urban species

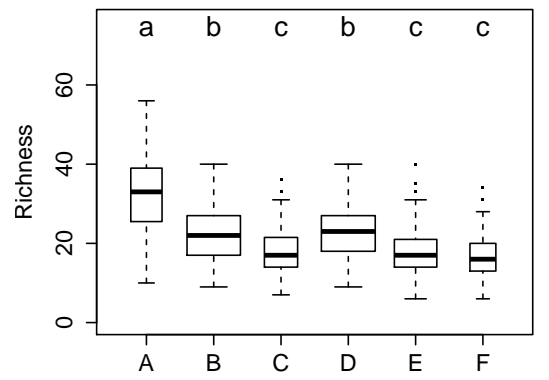

g) EIV species N 7-9

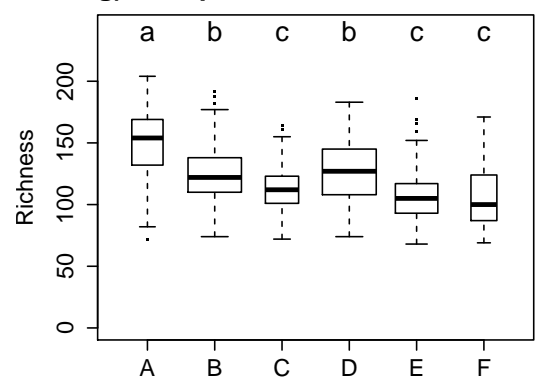

b) Threatened species

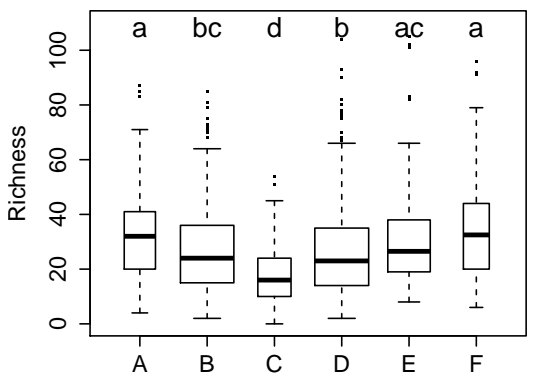

d) Native species

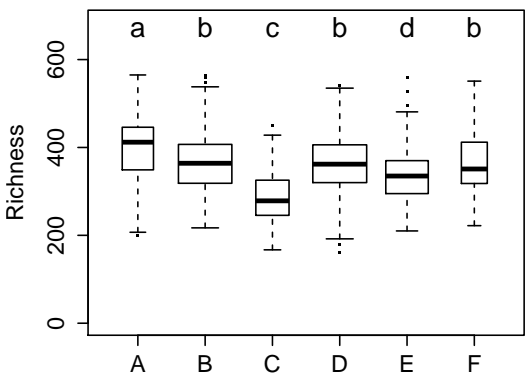

f) Forest species

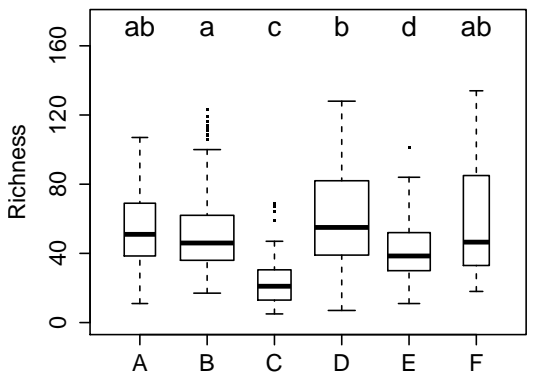

h) EIV species $\mathrm{N}$ 1-3

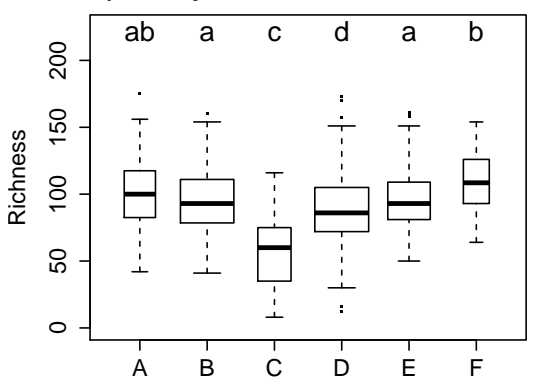

Figure 6.2: Differences in vascular plant species richness between different clusters resulting from k-means cluster analysis of 1383 landscape units (each c. $30 \mathrm{~km}^{2}$ ) in Lower Saxony, Germany (see also Table A.9 in Appendix A): (a) The total number of species recorded in Lower Saxony, (b) threatened species, (c) established neophytes, (d) native species, (e) urban species, (f) forest species, (g) species linked to nutrient-rich habitats (EIV species N 7-9) and (h) species linked to nutrient-poor habitats (EIV species N 1-3). Boxes are drawn with widths proportional to the square-roots of the number of observations in the groups; different letters indicate significant differences between clusters ( $\mathrm{p} \leq 0.05$, non-parametric Kruskal-Wallis test). 
Table 6.4: Scores of the first ordination axis of a PCA using the standardized landscape metrics calculated for 1383 landscape units in Lower Saxony, Germany. The proportion of urban areas (prop_urban) was found to be the most important variable to describe the underlying gradient, thus clusters were sorted using this variable.

\begin{tabular}{lr}
\hline Landscape metric & Scores PCA Axis 1 \\
\hline prop_urban & 0.37 \\
prop_traffic & 0.35 \\
all_SHDI & 0.35 \\
prop_industry & 0.29 \\
crop_NP & 0.27 \\
grass_NP & 0.26 \\
prop_grass & 0.21 \\
prop_water & 0.20 \\
all_ShapeAm & 0.11 \\
for_NP & 0.09 \\
prop_others & 0.09 \\
prop_crop & 0.04 \\
prop_forest & -0.20 \\
meshsize & -0.34 \\
all_AreaAm & -0.36 \\
\hline
\end{tabular}

\section{Discussion}

\section{Levels of landscape modification}

Our analysis revealed a gradient of landscape modification ranging from highly-fragmented and urbanised (cluster A) to little-fragmented landscapes with a large proportion of forests (cluster F). If one compares the particular clusters with the levels of landscape modification proposed by ForMAN \& GODRON (1986), cluster A is an 'urban landscape' as the landscape units of this cluster are dominated by artificial, man-made elements (Table 6.5). In contrast, cluster F resembles the 'managed landscape' of ForMAN \& Godron (1986) (Table 6.5). The landscape units of this cluster were chiefly covered by forests and hosted only a very small proportion of settlements. As Lower Saxony has a very long colonization history that affected and transformed all natural habitats such as primeval forest, bogs and marshland, no 'natural landscapes' according to FORMAN \& GODRON (1986) can nowadays be found.

In cluster B, all LCTs occurred at medium proportions and landscape fragmentation was quite high. The definition of 'suburban landscapes' by Forman \& GoDRON (1986) fits reasonably well to this cluster even if the landscape units were in several cases, but not necessarily, situated close to larger cities and, in the strict sense, are not 'suburbs'. Nevertheless, the authors define 'suburban landscapes' as "a town and country area with a heterogeneous, patchy mixture of residential areas, commercial centres, cropland, managed vegetation, and natural areas" (FormAN \& GoDron, 1986, p. 286), which is a good description of the situation found in the landscape units of this cluster. 
The naming of the following three clusters is more difficult. Cluster $\mathrm{C}$ was mainly characterised by a very large proportion of grasslands and water bodies. It dominates the coastal area as well as the north-western parts of the lowlands. Its landscape is highly transformed; most natural elements, i. e., marshlands and peat bogs that had been widespread in the past have been transformed by peat digging and drainage (BEHRE, 2008). The grasslands resulting from these landscape transformations are intensively managed and interspersed with drainage ditches. Cluster D, dominated by cropland, represents those parts of the study area with intensive agricultural use. Croplands prevailed in the flatter areas while ridges were usually covered by forests. The landscape of cluster E showed a patchy mosaic of cropland, grassland and forest with relatively few settlements. All three clusters correspond to the 'cultivated landscape' of Forman \& Godron (1986). This shows that the simple 5-level concept of 'landscape modification' proposed by FORMAN \& GODRON (1986) does not adequately differentiate our clusters when it comes to cultivated landscapes. The 10-level concept of landscape naturalness of MACHADO (2004) that allows for a more explicit differentiation seems to be more appropriate in our context. Following MaCHAdo (2004), the cultivated landscapes of our study may be thus better characterised as highly intervened (cluster C) to cultural assisted systems (cluster E; Table 6.5).

\section{Species richness patterns along the landscape modification gradient}

The clusters of landscape modification intensity found in this study were highly relevant for species richness patterns. Plant species numbers in all groups significantly differed between clusters. Species richness of the total number of species reported for Lower Saxony was found to peak in urban (cluster A) followed by suburban (cluster B) and managed landscapes (cluster F) whereas species richness was lowest in highly intervened landscapes (cluster $\mathrm{C}$ ). This trend was repeated by two of the species sub-groups (native and forest species) whereas species richness of low-nutrient and threatened plants followed a 'V shaped' trend with highest species numbers in urban and managed and lowest in highly intervened landscapes. Species richness of the remaining three sub-groups (neophytes, urban and high-nutrient plants) followed a downward trend throughout from urban to managed landscapes. These results confirm our third hypothesis that species numbers of different plant species groups show different patterns along the gradient of landscape modification. The results illustrate that it is important to consider not only the entire species pool of an area but rather a number of sub-groups selected using different criteria (cp. KowARIK, 1990).

The general peak in species numbers in urban (cluster A) and suburban landscapes (cluster B) observed for all species groups is unsurprising. Several authors have already highlighted the high species diversity of urban areas (KOWARIK, 1995; MILLER et al., 1997; HonNAy et al., 2003), which is mainly due to the higher landscape/habitat diversity (HonNAY et al., 2003; KüHN et al., 2004) and facilitated plant immigration in urban areas (KOWARIK, 1995). In contrast, low species numbers in all groups were 
found for the highly intervened landscape of cluster $C$. This is most likely a result of the cluster's low habitat diversity with its large proportion of transformed and intensively managed grassland areas interspersed with only few other LCTs and a low overall species diversity. The elevated species numbers in cluster D (cultural assisted to highly intervened system) reported for almost all species groups might be due to the spatial arrangement of the landscape units belonging to this cluster: Many of the landscape units are situated in the upland area and the eastern part of the lowlands with an overall larger species pool largely due to higher landscape heterogeneity (cp. SCHMIEDEL et al., 2011). Neophytes and high-nutrient plants might furthermore benefit from the large amount of intensively managed agricultural areas. The richness of forest species is also elevated in cluster D, which at first glance is surprising. This pattern may be explained by the fact that woodland patches suitable for forest plants are extant and widespread where agricultural land use was never, or is no longer, profitable.

The results show that the occurrence of non-native and urban species as well as species associated with nutrient-rich habitats is related to landscapes strongly influenced by urbanisation, fragmentation and eutrophication. For neophytes several studies confirm this trend (e.g. KowARIK, 1990; RoY et al., 1999; KüHN et al., 2003). By contrast, the number of native, threatened and even low-nutrient species is high in urban areas but also on the opposite side of the gradient, i. e., in the managed landscape less influenced by man. This is due to the aforementioned increased habitat diversity in urban areas that was shown to support even vulnerable species (HonNAy et al., 2003) and, regarding the other side of the landscape gradient, to the fact that largely unmodified landscapes are favourable to native plants and low-nutrient indicators (KowARIK, 1990; FIsCHer \& LindeNMAYER, 2007). LENZEN et al. (2009) reported that richness of threatened species was negatively related to the amount of croplands and permanent pastures, whereas the amount of non-arable land and natural forests was positively related to threatened plant species richness. Interestingly, forest species and species linked to nutrient-poor habitats follow the same trend and show relatively high species numbers in urbanised and highly fragmented landscapes. As for forest plants, apart from remaining woodland patches, they may find refuge in urban woods and parks. Plants of nutrient-poor environments with low competitive ability are found in urban areas on gravelly and abandoned sites such as railway areas and industrial fallows. In accordance with our results, HonNay et al. (2003) found the number of low-nutrient indicator species to be positively correlated with the amount of built-up area but did not give an explanation for this. 


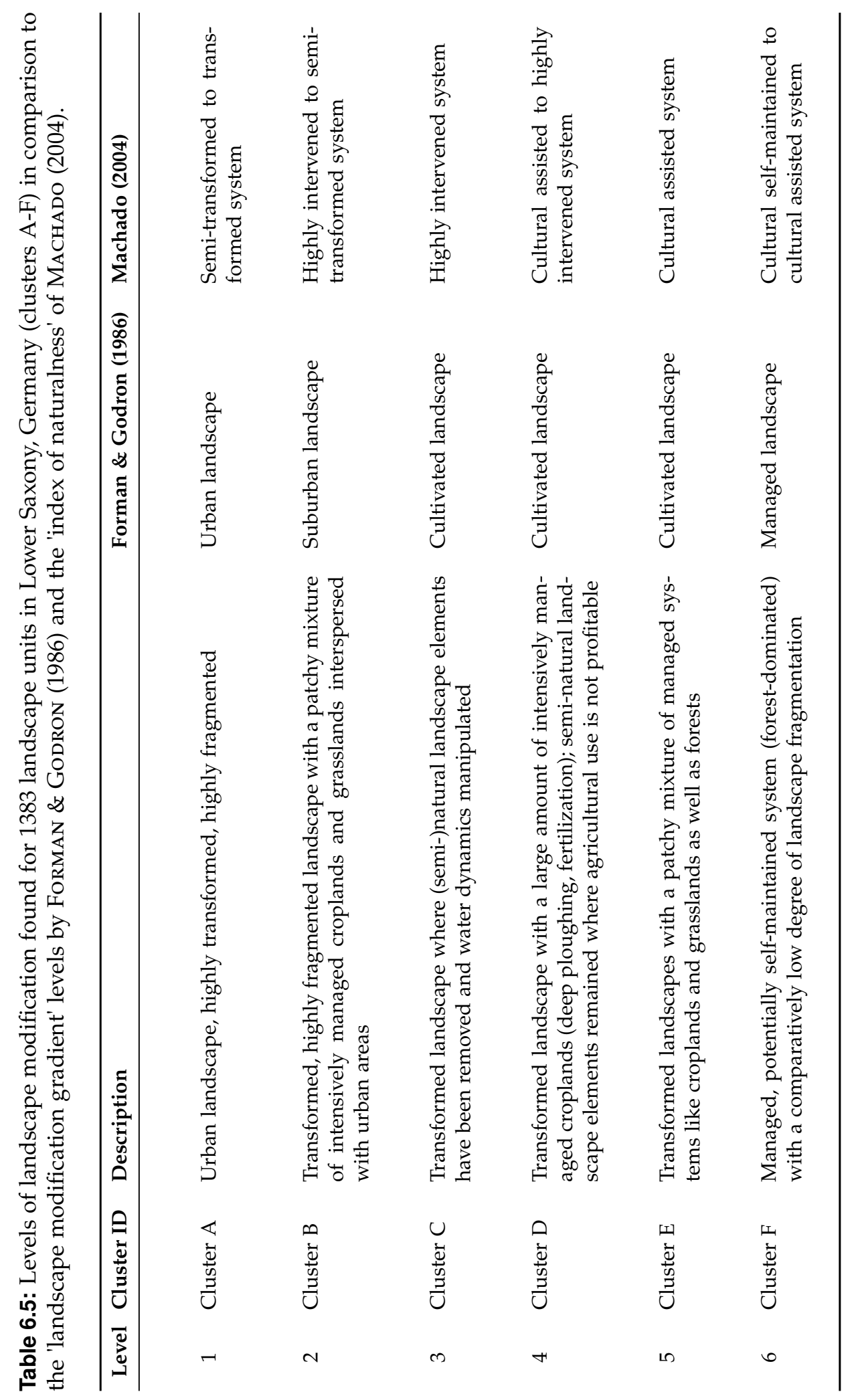




\section{Usefulness and transferability of the methodological approach}

To establish the levels of landscape modification, our approach simply applies information on landscape composition and configuration in combination with k-meansclustering. Thus, it involves neither a priori assumptions about the hemeroby or naturalness of the LCTs under consideration (as in WALZ \& STEIN, 2014) nor a subjective classification of levels of naturalness (as proposed by MACHADO, 2004). Therefore, the process of distinguishing levels of landscape modification in our concept is less susceptible to misinterpretation because only the results of the analysis and no intermediate steps need interpretation. As the levels of hemeroby of WALz \& STEIN (2014) were determined for the whole of Germany, including Lower Saxony, their results (especially those obtained for the $5 \mathrm{~km}$ grid) can be directly compared with those of our study. The comparison shows that the patterns derived from the two different approaches resemble each other. Both ways identify the human-dominated landscapes around the larger cities in contrast to the mainly forested areas in the southern and eastern parts of the state and designate areas under intense agricultural management.

When it comes to long-term monitoring of landscapes the concepts of WALz \& Stein (2014) and MAchado (2004) are superior to ours as their results may be easily compared for different time-steps as long as the same study units are used. In our approach, such results might not be directly comparable as changes in landscape composition and configuration will result in changes in the number and delimitation of clusters. Thus, our methodological approach may be applied when a 'snapshot' of the landscape status is needed and/or if due to a lack of more comprehensive information on landscape conditions more detailed assessments cannot be performed.

Similar to the concepts of WALz \& STEIN (2014) and MAchado (2004), our approach can easily be extended to other spatial scales. The ideal scale for an analysis can be selected that fits the particular research questions and/or the spatial resolution of the available base data. The resolution of our study was restricted by the size of the landscape units adopted by the plant survey of Lower Saxony. Higher resolution through smaller landscape units and more detailed differentiation of LCTs would increase the accuracy of the assessment. With enhancing differentiation of LCTs even small-scale habitats of potential importance for nature conservation (e.g. rocky outcrops, hedgerows) could be taken into account. Nevertheless, our study showed that interpretable results can be obtained with less detailed base data.

\section{Conclusions}

Despite the fact that our approach used simple landscape metrics and was conducted at a comparatively coarse scale of c. $30 \mathrm{~km}^{2}$, we were able to establish a 'gradient of landscape modification' that was shown to be meaningful for species richness. The gradient spans from highly fragmented, urbanised to less fragmented landscapes with a large proportion of forests. It was assessed without making a priori assump- 
tions about the hemeroby or naturalness of the LCTs under consideration. The simplicity of the approach makes it transferable to regions and countries for which there is no comprehensive information on landscape conditions. The methodology can easily be extended to other spatial scales. Those parts of the landscape which are most valuable for nature conservation can be identified and preselected. It should, however, be kept in mind that the coarse scale of the analysis leaves small-scale habitats of potential importance for nature conservation undetected. We could further show that it is important to employ a set of different species groups to avoid misleading conclusions drawn from richness patterns of a single possibly inadequate group. Suitable species groups to be considered should include indicator and threatened species important for nature conservation as well as species that might pose a threat to other species or ecosystems. As high-resolution data on recent land cover (for an overview see Manakos \& BRAUN, 2014) and the distribution of vascular plant species are becoming increasingly available in more and more countries (see Table 6 in CulMsEe et al., 2014) our approach is easily applicable to other large areas in Europe.

\section{Acknowledgement}

Funding of the project "Identification of indicator species groups of grassland and forest habitats for biodiversity monitoring and evaluation" by the German Federal Foundation for the Environment, grant number DBU 26752, is gratefully acknowledged. We would like to thank all volunteers who reported vascular plant species records in Lower Saxony, and the Lower Saxony Water Management, Coastal Defence and Nature Conservation Agency (NLWKN) for providing these data. We thank Laura Sutcliffe for linguistic revision. 


\section{References}

Bastian, O. \& Bernhardt, A. (1993): Anthropogenic landscape changes in Central Europe and the role of bioindication. Landscape Ecology 8: 139-151.

BEHRE, K.-E. (2008): Landschaftsgeschichte Norddeutschlands: Umwelt und Siedlung von der Steinzeit bis zur Gegenwart. Wachholtz, Neumünster. 300 pp.

BfN (Bundesamt für Naturschutz) (2013): FloraWeb: Daten und Informationen zu Wildpflanzen und zur Vegetation Deutschlands. URL http:/ / www.floraweb.de. Retrieved 07/01/2013.

Brock, G.; Pihur, V.; Datta, S. \& Datta, S. (2008): clValid: An R package for cluster validation. Journal of Statistical Software 25.

Culmsee, H.; Schmidt, M.; Schmiedel, I.; Schacherer, A.; Meyer, P. \& Leuschner, C. (2014): Predicting the distribution of forest habitat types using indicator species to facilitate systematic conservation planning. Ecological Indicators 37: 131-144.

DAILY, G. C. (2001): Ecological forecasts. Nature 411: 245.

Deutschewitz, K.; Lausch, A.; KühN, I. \& Klotz, S. (2003): Native and alien plant species richness in relation to spatial heterogeneity on a regional scale in Germany. Global Ecology and Biogeography 12: 299-311.

DisE, N. B. (2011): Nitrogen as a threat to European terrestrial biodiversity. In: SUTton, M. A.; Howard, C. M.; Erisman, J. W.; Billen, G.; Bleeker, A.; Grennfelt, P.; van Grinsven, H. \& Grizzetti, B. (eds.) The European nitrogen assessment, Cambridge University Press, Cambridge, UK and New York. pp. 463-494.

Drachenfels, O. v. (2010): Überarbeitung der Naturräumlichen Regionen Niedersachsens. Informationsdienst Naturschutz Niedersachsen 30: 249-252.

Fischer, J. \& LindenMAYeR, D. B. (2007): Landscape modification and habitat fragmentation: a synthesis. Global Ecology and Biogeography 16: 265-280.

Foley, J. A.; DeFries, R.; Asner, G. P.; Barford, C.; Bonan, G.; Carpenter, S. R.; Chapin, F. S.; Coe, M. T.; Daily, G. C.; Gibbs, H. K.; Helkowski, J. H.; Holloway, T.; Howard, E. A.; Kucharik, C. J.; Monfreda, C.; Patz, J. A.; Prentice, I. C.; Ramankutty, N. \& SNyder, P. K. (2005): Global consequences of land use. Science 309: 570-574.

Forman, R. T. T. \& Godron, M. (1986): Landscape ecology. John Wiley \& sons, New York. $644 \mathrm{pp}$.

GARVE, E. (2004): Rote Liste und Florenliste der Farn- und Blütenpflanzen in Niedersachsen und Bremen. Informationsdienst Naturschutz Niedersachsen 24: 1-76. 
GARVE, E. (2007): Verbreitungsatlas der Farn- und Blütenpflanzen in Niedersachsen und Bremen. Naturschutz und Landschaftspflege in Niedersachsen 43: 1-507.

Giraudoux, P. (2013): Package 'pgirmess': Data analysis in ecology. Version 1.5.8.

Grabherr, G.; Koch, G.; Kirchmeir, H. \& Reiter, K. (1998): Hemerobie österreichischer Waldökosysteme, vol. 17. Universitätsverlag Wagner, Innsbruck. 493 pp.

Harper, K. A.; Macdonald, S. E.; Burton, P. J.; Chen, J. Q.; Brosofske, K. D.; SaunDers, S. C.; EusKirchen, E. S.; Roberts, D.; JaIteh, M. S. \& EsSEen, P. A. (2005): Edge influence on forest structure and composition in fragmented landscapes. Conservation Biology 19: 768-782.

Henning, C. (2014): Package 'fpc': Flexible procedures for clustering. URL http: //cran.r-project.org/web/packages/fpc/index.html.

Hill, M. O.; Roy, D. B. \& Thompson, K. (2002): Hemeroby, urbanity and ruderality: bioindicators of disturbance and human impact. Journal of Applied Ecology 39: 708720.

Honnay, O.; Piessens, K.; Landuyt, W. v.; Hermy, M. \& Gulinck, H. (2003): Satellite based land use and landscape complexity indices as predictors for regional plant species diversity. Landscape and Urban Planning 63: 241-250.

Jalas, J. (1955): Hemerobe und hemerochore Pflanzenarten. Ein terminologischer Reformversuch. Acta Societatis pro Fauna et Flora Fennica 72: 1-15.

JAnsen, F.; Zerbe, S. \& Succow, M. (2009): Changes in landscape naturalness derived from a historical land register - a case study from NE Germany. Landscape Ecology 24: $185-198$.

Kerr, J. T. \& Deguise, I. E. (2004): Habitat loss and the limits to endangered species recovery. Ecology Letters 7: 1163-1169.

KLOTZ, S. \& KüHN, I. (2002): Indikatoren des anthropogenen Einflusses auf die Vegetation. Schriftenreihe für Vegetationskunde 38: 241-246.

KLOtZ, S.; KüHN, I. \& DuRKA, W. (2002): BIOLFLOR - Eine Datenbank zu biologischökologischen Merkmalen der Gefäßpflanzen in Deutschland. Schriftenreihe für Vegetationskunde 38: 1-334. URL http://www.biolflor.de.

KowARIK, I. (1988): Zum menschlichen Einfluß auf Flora und Vegetation: Theoretische Konzepte und ein Quantifizierungsansatz am Beispiel von Berlin (West). Techn. Univ., Berlin. 280 pp.

KowARIK, I. (1990): Some responses of flora and vegetation to urbanization in Central Europe. In: SuкорP, H.; HejnÝ, S. \& Kowarik, I. (eds.) Urban ecology, SPB Academic Publishing, Den Hague, Netherlands. pp. 45-74. 
KOWARIK, I. (1995): On the role of alien species in urban flora and vegetation. In: Pyšek, P.; Prach, K.; RejmaneK, M. \& Wade, M. (eds.) Plant invasions, SPB Academic Pub., Amsterdam. pp. 85-103.

KüHN, I.; BRAndL, R. \& Klotz, S. (2004): The flora of German cities is naturally species rich. Evolutionary Ecology Research 6: 749-764.

KühN, I.; Brandl, R.; MaY, R. \& KLOTZ, S. (2003): Plant distribution patterns in Germany - Will aliens match natives? Feddes Repertorium 114: 559-573.

LANG, S. \& BlaschKe, T. (2007): Landschaftsanalyse mit GIS. Ulmer, Stuttgart. 404 pp.

Lenzen, M.; Lane, A.; Widmer-Cooper, A. \& Williams, M. (2009): Effects of land use on threatened species. Conservation Biology 23: 294-306.

LGN (Landesvermessung und Geobasisinformation Niedersachsen) (2008): Amtliches Topographisch-Kartographisches InformationsSystem (ATKIS): Updated 17 December 2008.

LIIRA, J. \& SEPP, T. (2009): Indicators of structural and habitat natural quality in boreo-nemoral forests along the management gradient. Annales Botanici Fennici 46: 308-325.

LucK, M. \& WU, J. (2002): A gradient analysis of urban landscape pattern: a case study from the Phoenix metropolitan region, Arizona, USA. Landscape Ecology 17: 327-339.

Machado, A. (2004): An index of naturalness. Journal for Nature Conservation 12: 95-110.

ManaKos, I. \& Braun, M. (2014): Land use and land cover mapping in Europe: Practices \& trends, vol. 18. Springer, Dordrecht. 441 pp.

Mander, Ü.; Palang, H. \& Ihse, M. (2004): Development of European landscapes. Landscape and Urban Planning 67: 1-8.

Mascarua lopez, L. E.; Harper, K. A. \& Drapeau, P. (2006): Edge influence on forest structure in large forest remnants, cutblock separators, and riparian buffers in managed black spruce forests. Ecoscience 13: 226-233.

McGarigal, K.; Cushman, S. A.; Neel, M. C. \& Ene, E. (2002): FRAGSTATS: Spatial pattern analysis program for categorical maps. Version 3. URL www.umass.edu/ landeco/research/fragstats/fragstats.html.

Meeus, J. (1995): Pan-European landscapes. Landscape and Urban Planning 31: 57-79. 
Miller, J. N.; Brooks, R. P. \& Croonquist, M. J. (1997): Effects of landscape patterns on biotic communities. Landscape Ecology 12: 137-153.

Moser, B.; Zechmeister, H. G.; Plutzar, C.; Sauberer, N.; Wrbka, T. \& Grabherr, G. (2002): Landscape patch shape complexity as an effective measure for plant species richness in rural landscapes. Landscape Ecology 17: 657-669.

Nabe-Nielsen, J.; Sibly, R. M.; Forchhammer, M. C.; Forbes, V. E.; Topping, C. J. \& RANDS, S. (2010): The effects of landscape modifications on the long-term persistence of animal populations. PLoS ONE 5: e8932.

NLfB (Niedersächsisches LANdesAMt FÜr Bodenforschung) (1997): Böden in Niedersachsen, Teil 1: Bodeneigenschaften, Bodennutzung und Bodenschutz. URL http://www.lbeg.de/extras/nlfbook/html/nds_main.htm. Retrieved 07/09/2010.

Olsen, L. M.; Dale, V. H. \& Foster, T. (2007): Landscape patterns as indicators of ecological change at Fort Benning, Georgia, USA. Landscape and Urban Planning 79: 137-149.

R Development Core Team (2012): R: A language and environment for statistical computing. URL http://www.R-project.org.

Roy, D. B.; Hill, M. O. \& Rothery, P. (1999): Effects of urban land cover on the local species pool in Britain. Ecography 22: 507-515.

Sala, O. E.; Chapin, F. S.; Armesto, J. J.; Berlow, E.; Bloomfield, J.; Dirzo, R.; Huber-Sanwald, E.; Huenneke, L. F.; Jackson, R. B.; Kinzig, A.; Leemans, R.; Lodge, D. M.; Mooney, H. A.; Oesterheld, M.; Poff, N. L.; Sykes, M. T.; Walker, B. H.; WALKeR, M. \& WALL, D. H. (2000): Global biodiversity scenarios for the year 2100. Science 287: 1770-1774.

Schmidt, M.; Kriebitzsch, W.-U. \& Ewald, J. (2011): Waldartenlisten der Farn- und Blütenpflanzen, Moose und Flechten Deutschlands. BfN-Skripten 299: 1-111.

Schmiedel, I. \& Culmsee, H. (submitted): The influence of landscape fragmentation, expressed by the 'effective mesh size index', on regional patterns of vascular plant species richness in Lower Saxony, Germany .

Schmiedel, I.; Schacherer, A.; HaucK, M.; Schmidt, M. \& Culmsee, H. (2011): Verbreitungsmuster der Farn- und Blütenpflanzen in Niedersachsen und Bremen unter Berücksichtigung ihres Einbürgerungsstatus und ihrer Gefährdungssituation. Tuexenia 31: 211-226.

Steinhardt, U.; Herzog, F.; Lausch, A.; Müller, E. \& Lehmann, S. (1999): Hemeroby index for landscape monitoring and evaluation. In: РүKH, Y. A.; НүATT, D. E. \& LENZ, R. J. (eds.) Environmental indices systems analysis approach, EOLSS, Advances in sustainable development. pp. 237-257. 
Stoate, C.; Boatman, N.; Borralho, R.; Carvalho, C. R.; Snoo, G. R. D. \& Eden, P. (2001): Ecological impacts of arable intensification in Europe. Journal of Environmental Management 63: 337-365.

Sukopp, H. (1976): Dynamik und Konstanz in der Flora der Bundesrepublik Deutschland. Schriftenreihe für Vegetationskunde 10: 9-27.

VEAC (Victorian Environmental Assessment Council) (2010): Remnant native vegetation investigation: Discussion paper. URL http://www.veac.vic.gov.au/ documents/Discussion\%20Paper\%20-\%20Full\%20Report\%20RNV.pdf. Retrieved $01 / 11 / 2014$.

WALz, U. \& STEIN, C. (2014): Indicators of hemeroby for the monitoring of landscapes in Germany. Journal for Nature Conservation 22: 279-289.

Wesche, K.; Krause, B.; Culmsee, H. \& Leuschner, C. (2012): Fifty years of change in Central European grassland vegetation: Large losses in species richness and animal-pollinated plants. Biological Conservation 150: 76-85.

Winter, S.; Fischer, H. S. \& Fischer, A. (2010): Relative quantitative reference approach for naturalness assessments of forests. Forest Ecology and Management 259: 1624-1632.

Wrbka, T.; Erb, K.-H.; Schulz, N. B.; Peterseil, J.; Hahn, C. \& Haberl, H. (2004): Linking pattern and process in cultural landscapes. An empirical study based on spatially explicit indicators. Land Use Policy 21: 289-306.

Yu, X. \& NG, C. (2006): An integrated evaluation of landscape change using remote sensing and landscape metrics: a case study of Panyu, Guangzhou. International Journal of Remote Sensing 27: 1075-1092. 
Synthesis 
The analyses performed in the context of the present thesis aimed at improving the understanding of the relationship between species richness patterns and their underlying processes at the landscape scale with special emphasis on human influences. In particular, the analyses (a) identified and described the patterns of vascular plant species richness in the federal states of Lower Saxony and Bremen, Germany (chapter 3 ), (b) evaluated the adequacy of the protected area network for the protection of rare and threatened plant species in the lowland region of the study area (chapter 4) and (c) analysed the relationship between richness patterns and the human influence on the landscape (chapter 5 and 6).

The following sections recapitulate the key findings of the four main chapters of the thesis (section 7.1) and review the transferability of the methodologies applied in the thesis to other study regions (section 7.2). Furthermore, the limitations of landscape-level approaches are discussed (section 7.3). The chapter closes with concluding remarks and the open questions that build on this research (section 7.4).

\subsection{Key findings and implications}

\subsubsection{Distinct patterns of vascular plant species richness in Lower Saxony and Bremen}

The analysis presented in chapter 3 revealed a heterogeneous distribution of species richness across the study area for all six species groups considered in the study. Species numbers showed a remarkable increase from north to south and - to some extent - also from west to east. The spatial distribution of the centres of plant species richness of the different groups largely correlated with each other. However, also some interesting differences in these patterns were observed. Richness patterns of all species established in the study area largely corresponded to the patterns found for native and non-threatened species. An aggregated distribution with high overall species numbers were reported for the upland area and - with the exception of the major stream valleys of the Weser, Aller and Elbe - a much lower overall richness in the coastal and lowland area. Archaeophyte richness was particularly concentrated in the coastal area of Bremen, the Weser and Aller plains, and the loess landscapes of the lowland area ${ }^{1}$ whereas the richness of established neophytes was highest in urban centres.

Richness patterns of threatened plants - in contrast to the patterns of all other groups - were found to be spatially highly differentiated: at the coast only the islands in the North Sea represented small hotspots, while in the lowlands relatively large hotspots were situated in the Wendland, the Luneburg Heath and the Elbe-Weser triangle north of Bremen. In the uplands, the area around Göttingen, the uplands of

\footnotetext{
${ }^{1}$ German: Börden
} 
the Weser and Leine valleys and the margins of the Harz Mountains represented well defined hotspots of Red List species.

A general trend that was observed across all species groups was that grid cells inside which urban centres were situated were found to be particularly rich in species. This relationship was especially evident for neophytes but weak for threatened species.

The presented approach can support systematic conservation planning by identifying landscape units rich in species that should receive particular attention. However, in doing so not only the total number of species but also different groups of species should be considered (cp. subsection 7.1.4). Haeupler \& Vogel (1999) and Haeupler (2000) suggest that hotspots of threatened plant species are able to reveal the "real" hotspots of vascular plant species richness and thus provide a suitable species group to be used in the determination of landscape units of high conservation value (DUELLI \& OвRIST, 2003). Indeed, richness patterns of threatened species were found to be spatially much more differentiated than those of the other species groups considered in the analysis.

\subsubsection{Effective 'territorial' protection of hotspots of threatened plant species}

In contrast to the results of similar studies conducted for different regions of the world that identified numerous gaps in existing protected area networks (see e.g. Scott et al., 2001; Andelman \& Willig, 2003; Deguise \& KerR, 2006; Rodrigues et al., 2004), the analysis on the effectiveness of the protected area network for the conservation of threatened plant species in the lowland region of the study area confirmed a good overall coverage of the hotspots of threatened species. In particular, richness centres of the total number of threatened species were covered by nature protection areas in $85 \%$ of the 41 geographical sub-regions considered in the analysis. The proportion of well covered hotspots increased to an effective protection in $95 \%$ of the geographical regions when including Natura 2000 sites into the analysis. A repetition of the analyses for threatened species linked to forest habitats showed that richness centres of these species were effectively protected by nature protection areas in only $60 \%$ of the regions; an inclusion of Natura 2000 sites increased the proportion to $81 \%$. However, the proven 'territorial' protection of these hotspots will only be able to preserve viable populations of the species if the protected areas are managed in an appropriate way. For many of these areas, legal regulations controlling the management of the areas are expressed very vaguely ${ }^{2}$ or their development is still under way ${ }^{3}$. To effectively protect viable populations of species in the future, these regulations should be adjusted to be able to support the particular species of conservation concern. Repetitive studies in future years will then need to show if the effective 'territorial' protection is resulting in an effective species protection.

\footnotetext{
${ }^{2}$ This is especially the case for nature reserves (Naturschutzgebiete).

${ }^{3}$ This is mainly the case for Natura 2000 sites as these areas have been designated quite recently.
} 
As many protected areas are isolated and small in size their effectiveness in supporting species might be limited. Therefore, areas outside reserves should also be managed in a way that supports threatened species, i.e. a "dual conservation strategy" according to JACKSON et al. (2009) should be pursued.

The methodology applied in the study was shown to be suitable to evaluate the effective 'territorial' protection of hotspots of threatened species. By transferring it to other regions with little information on the adequacy of the protected area network, it can contribute to systematic conservation planning.

\subsubsection{Human impact matters: Plant species richness along gradients of landscape modification and fragmentation}

The relationship between species richness patterns and human activities at the landscape scale was studied by evaluating the influence of the degree of landscape modification in general and landscape fragmentation in particular. The following two subsections highlight the key findings of chapters 5 and 6 dealing with these topics.

The Effective Mesh Size Index as an ecologically meaningful measure of landscape fragmentation The analysis presented in chapter 5 of the thesis demonstrated that the Effective Mesh Size Index $\left(m_{e f f}\right)$ can act as an ecologically meaningful measure of landscape fragmentation as long as relevant fragmentation geometries are chosen. Landscape fragmentation expressed by $m_{e f f}$ in the study area was found to vary largely between landscape units and was identified to significantly affect the richness of almost all plant species groups considered in the analysis. The size of the effect differed considerably between the groups and was largest for neophytes followed by archaeophytes. In contrast, richness patterns of native species were only slightly affected whilst those of threatened species were not affected. Instead, the variation partitioning approach for the latter species groups clearly highlighted the importance of the effect of environmental factors.

All species groups, except threatened species, showed a negative correlation with $m_{\text {eff }}$, i. e. richness was highest in highly fragmented and lowest in least fragmented grid cells.

In the analysis, five different $m_{e f f}$ types calculated using different fragmentation geometries were tested. Out of these, only two were found to be significant in explaining species richness patterns, namely those that used urban-industrial areas, traffic infrastructure and water bodies as fragmenting elements.

The findings of the study support the untested assumption of several researchers (e. g. JAEger, 2000; JAEgER et al., 2008 and GiRvetz et al., 2008) that $m_{e f f}$ is an ecologically relevant measure of landscape fragmentation. The presented approach might help future studies to determine correct fragmentation geometries to use with $m_{\text {eff }}$ and might facilitate the unravelling of fragmentation impacts at the landscape scale. As $m_{\text {eff }}$ in Germany is regularly assessed as one of two national indicators of land- 
scape fragmentation in context of the National Strategy on Biodiversity the results obtained in the analysis are especially relevant as a contribution to future assessments.

Variation in species richness along a gradient of landscape modification Chapter 6 of this thesis applied simple information on land cover and easily measurable landscape metrics to determine a 6-level gradient of landscape modification spanning from highly fragmented urban landscapes to little fragmented ones with a large proportion of forests. Species richness of the eight groups of vascular plants considered in the analysis significantly differed along the gradient. For the entire and native species pool as well as for forest, low-nutrient indicating and threatened plants, species numbers peaked at the opposing ends of the modification gradient. For neophytes, urban plants and high-nutrient indicators, richness decreased along the gradient from high species numbers in urbanised to low numbers in less modified landscapes.

The study showed that concepts of landscape modification can be successfully linked to measures of species richness/diversity that were tested empirically here for the first time. This finding is of particular importance as concepts describing the human influence on the landscape in Germany are planned to be integrated into the nationwide monitoring system (WALz \& STEIN, 2014). In this context, knowledge on the actual relevance of such concepts for biodiversity might therefore further increase their usefulness.

The strength of the analysis is, that the assessment of the landscape modification gradient neither involved a priori assumptions about the hemeroby or naturalness of the land cover types under consideration (as in WALz \& STEIN, 2014) nor a subjective classification of levels of naturalness (as proposed by MACHADO, 2004). Therefore, the process of distinguishing levels of landscape modification is less susceptible to misinterpretation than the concepts of WALZ \& STEIN (2014) and Machado (2004) because only the results of the analysis and no intermediate steps need interpretation. Due to its coarse scale, the analytical approach is transferable to other regions and countries for which there is no comprehensive information on landscape conditions. Following interpretation by experts, the methodology developed in the study allows for the identification and preselection of those parts of the landscape that are most valuable for nature conservation.

\subsubsection{Overall key findings derived from the studies}

In addition to the individual key findings of the four studies presented above, two further overall findings of all the analyses conducted in the present thesis can be derived that will be discussed below.

Habitat heterogeneity as a key driver of species richness in urban areas? A general emerging trend that was observed in the conducted studies on landscape fragmen- 
tation and modification but also in the first study on species richness patterns, was the relationship between species richness and urban land cover: The latter together with the degree of landscape fragmentation which is usually tightly linked with the degree of urbanisation were both found to be positively associated with the richness of all species groups. The only exception being the richness of threatened species that was found to be independent of landscape fragmentation. Therefore no direct negative effects of human land use on species richness patterns could be identified. However, these results should not be used to conclude that intensive human land use increases the richness of species ${ }^{4}$. Instead, it seems that the elevated habitat heterogeneity of urban areas as proposed by several researchers (HAEUPLER, 1974; KüHN et al., 2003; WANIA et al., 2006; LIPPE \& KOWARIK, 2008) balances or even outbalances the assumed negative effect of landscape modification and fragmentation as long as suitable habitats are not completely removed (cp. HonnAy et al., 2003). Therefore it can be concluded that it is important to conserve the high habitat diversity of urban areas and to prevent their homogenisation.

Quality vs. quantity: avoiding drawing the wrong conclusions The analyses conducted in the framework of the thesis produced results that differed considerably between the various species groups examined in the individual studies. The richness of the groups showed different patterns according to the distribution in the study area, the coverage by protected areas and the relationship with landscape modification and fragmentation. These findings clearly emphasise the importance of considering not one single but rather an array of different species groups using various criteria. By doing so, not only quantitative but also qualitative aspects can be considered (cp. subsection 7.1.2) which are not biased to protecting mainly easily recorded or common species at the cost of a wider range of biodiversity (cp. KowARIK, 1990; Duelli \& Obrist, 2003; Schetter et al., 2013). In this context, for example, the residence or threat status of species ${ }^{5}$, their habitat preferences ${ }^{6}$ or a combination of these criteria ${ }^{7}$ can be used to assemble groups of species.

\subsection{Transferability of the methodological approach}

The analyses applied in the framework of the present thesis were conducted on a regional scale covering the area of Lower Saxony and Bremen. For this area detailed information on species occurrences and data on environmental conditions and landscape composition as well as configuration were available.

\footnotetext{
${ }^{4}$ Extensive management as applied in traditional cultural (pre-industrial) landscapes, in turn, is known to be beneficial for biodiversity (EEA, 2004; ANGELsTAM, 2006)

${ }^{5}$ As applied in Schmiedel et al. (2011, 2013, submitted) and Schmiedel \& CulmseE (submitted).

${ }^{6}$ As applied in Schmiedel et al. (2013) and Schmiedel et al. (submitted).

${ }^{7}$ As applied in SCHMIEDEL et al. (2013).
} 
If suitable base data are at hand, the methodological approaches applied in the present thesis can be transferred to other - even larger - areas. The most important (and often the rarest) data in this context are area-wide species records. The records need to be reported on a grid- or point-basis ${ }^{8}$ and have to be of high quality (cp. subsection 7.3). Additionally, data on land cover and the abiotic environment are needed.

Transferability to Germany and its federal states For most of the federal states of Germany, data comparable to those used in this thesis are available that would allow a transfer. High-resolution data on land cover and topography in this thesis were taken from ATKIS-DLM and ATKIS-DEM (see section 2.2) which are both available for the whole country. Climatic and soil data can be obtained in high resolution for every federal state from the German Weather Service and the regional authorities, respectively. Concerning species records, most federal states maintain their own mapping projects (see Table 7.1). The resolutions of these mapping approaches are similar to that used for Lower Saxony or even higher (e.g. c. $8 \mathrm{~km}^{2}$ grid cells for North Rhine-Westphalia, Saxony and Thuringia), the latter enabling even more detailed assessments. However, the completeness and reliability of the floristic datasets resulting from these projects would have to be tested before using them in further analyses.

With the distribution atlas of the vascular plants of Germany having been published recently (NeTPhyD \& BFN, 2013) reliable data on species occurrences are also available for the whole of Germany. Species data of this database are reported at the same resolution as the data for Lower Saxony (Messtischblatt-Quadranten, 1/4 of a topographical map). Therefore, the methodologies applied in this thesis are directly transferable to the whole country area.

Transferability to European countries Similarly to Germany, most countries in Europe maintain species mapping projects. Out of the projects listed in Table 7.1 for data acquisition only Poland uses a grid less precise than that utilised in this thesis. All other countries apply more or less equal or even higher resolutions than used in this study. As suggested for species data of the federal states of Germany, prior to an analysis, it needs to be checked whether the available information is reliable and complete.

Additional information on land cover ${ }^{9}$ and environmental conditions should be available for most European countries and therefore methods might be transferred to these areas as well.

The database of the Atlas Flora Europaea (Jalas \& SuOMInen, 1972-2013) that covers about $20 \%$ of the European flora (Finnish Museum of Natural History, 2014) pro-

\footnotetext{
${ }^{8}$ Point data can be transferred to grid-based data of varying resolutions.

${ }^{9}$ For an overview on existing data on land cover in Europe see MANAKOS \& BRAUN (2014).
} 


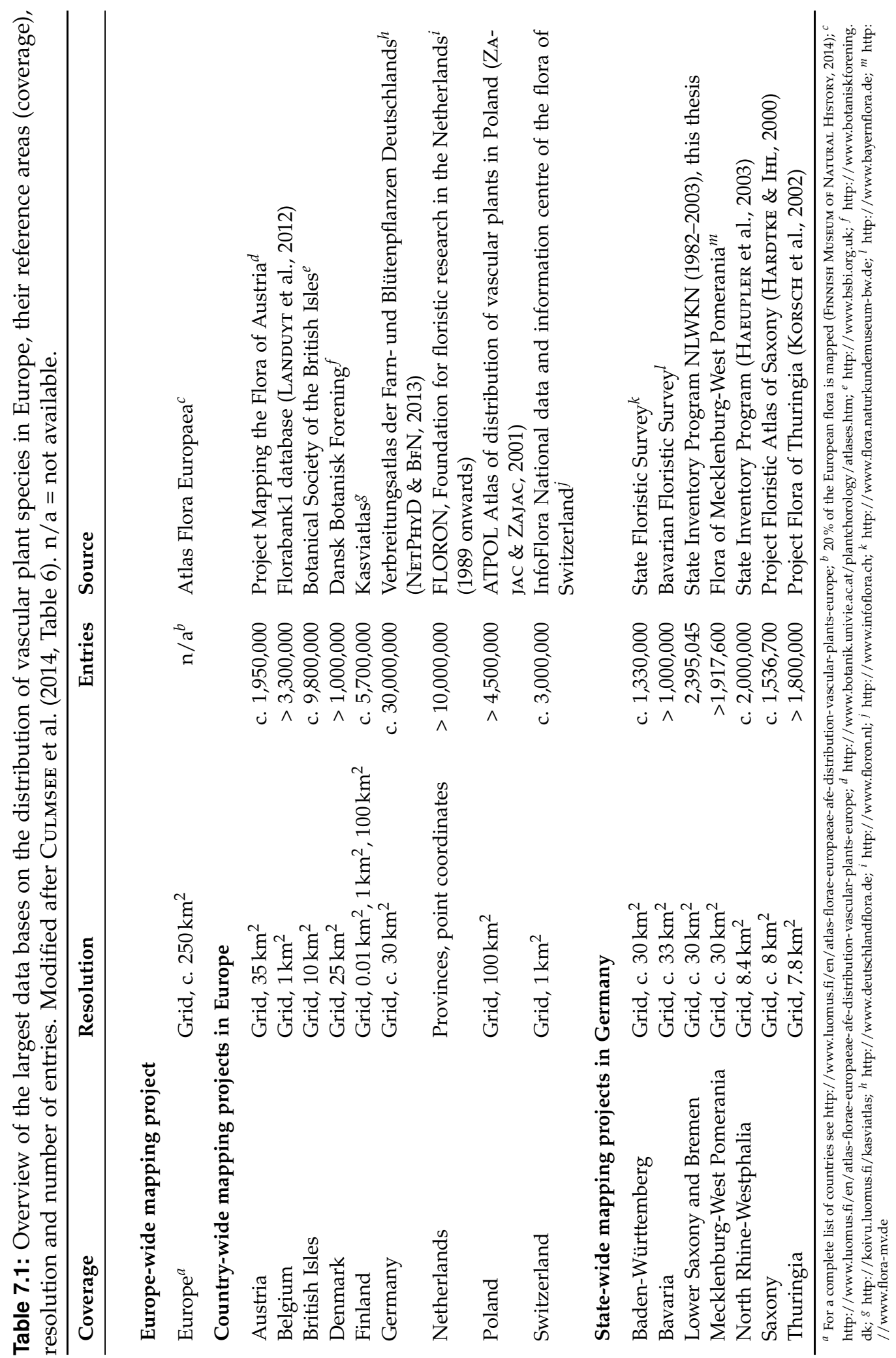


vides data on species distributions for the whole of Europe on a grid of c. $250 \mathrm{~km}^{2}$, i. e. a resolution much coarser than that used in the present thesis. Moreover, highresolution data on land cover for the whole area of Europe are not as easy to obtain as for the particular countries. However, information on land cover on this scale could be derived from more coarse-scaled data available for the overall area. 'Corine Land Cover 2006' (EEA, 2013) in this context provides a remote-sensing-derived raster data set on land cover with a pixel size of $100 \mathrm{~m} \times 100 \mathrm{~m}$ that can be used for research purposes free of charge. Information on topography and climatic conditions available on a resolution of $1 \mathrm{~km} \times 1 \mathrm{~km}$ can be derived from Worldclim data (Hijmans et al., 2013). Therefore, potentially suitable data are available that could form the basis of analyses similar to that applied in this thesis. Because of the large differences in data resolution the results obtained from such analyses would probably, however, vary largely from those obtained at the regional scale.

Transferability to countries outside Europe The availability of high-resolution plant records for countries outside Europe is limited. However, for some areas, e.g. the state of California, USA (The Calflora Database, 2014) grid-based species records are acquirable. For other states of the USA (e. g. Alabama, New York, Florida, see ALabama Herbarium Consortium, 2014; New York Flora Association, 2014; UniVERSITY OF Florida, INSTITUTE For Systematic Botany, 2014) as well as for China (YANG et al., 2013) such information are not available on a grid basis but on countylevel only.

Information on species records from other countries unfortunately are very hard to obtain. The transferability of the methods applied in this thesis to areas outside of Europe is therefore limited.

\subsection{Limitations of landscape-level approaches}

Even if frequently shown to be very powerful, landscape-level approaches are subjected to several inaccuracies arising from the scale chosen for the particular analysis, the inclusion of biased base data and inaccuracies provoked by generalisations needed to perform the analyses. Gotelli et al. (2009) suggest that a large number of potential sources of error are linked with each data layer included in the analysis.

\subsubsection{Bias due to generalisations, data resolutions and spatial scale}

The representation of 'real world' conditions and patterns in 'computer space' is always subject to necessary generalisations (i.e. delimitation and discretisation processes) that help to pool the vast amount of available information. It is important that the magnitude of generalisation applied to the data should match the processes under consideration (LANG \& BLASCHKE, 2007). The ATKIS-DLM data used in the present thesis represent a good example for generalisations: The DLM defines hard 
boundaries along more or less homogeneous landscape elements that in most cases can not fully reflect the real conditions, as the boundaries between many land cover types (especially natural habitats) are usually more continuous. Because the latter cannot be represented cartographically and furthermore would provoke problems in data analysis, the hard boundaries are accepted as the best approximation of reality (Green \& Hartley, 2000).

Generalisations affect both the spatial and the thematic resolution of the data. The latter describes the way in which the geo-spatial data are categorised ${ }^{10}$. The spatial resolution of an analysis, in turn, is described by the extent of the study area as well as the grain size ${ }^{11}$. It was shown to affect all levels of data, i.e. species, land cover and environmental data.

In this context, several studies have shown that patterns of species richness/diversity change with the scale of observation or analysis (PALMER \& WHITE, 1994; He et al., 2002; Kallimanis et al., 2008) and therefore "areas of high species richness at one scale may appear of low species richness at the other scale" (KALLIMANIS et al., 2008, p. 148). This particular behaviour is attributable to the fact that species richness is non-additive when aggregated across different spatial scales ( $\mathrm{He}$ et al., 2002).

Most studies conducted at the landscape scale make use of land cover data. Usually, landscape metrics in this context are used to quantify the composition and configuration of a landscape unit. As these indices are assumed to be strongly scale-dependent, a lot of attention has focussed on the behaviour of the metrics across changing thematic and spatial resolutions. Within these studies, the thematic resolution was found to largely affect the outcome of most landscape metrics (BAILEY et al., 2007; BUYANTUYEV \& WU, 2007). Related to this, the usefulness of the indices in describing landscape characteristics at varying thematic resolutions was found to largely depend on resolution: Whilst some indices are more useful at a coarser thematic resolution others are more suitable at finer scales (BAILEY et al., 2007). Similarly, only few landscape metrics have been identified to behave consistently across different spatial resolutions (Turner et al., 1989; SAURA \& MARTinez-Millán, 2001; Wu, 2004). In this context, TURNer et al. (1989) identified an increase in grain size to be responsible for the loss of rare and small-scale land cover types in the landscape. ScHINDLER et al. (2013) studied the performance of landscape metrics as indicators of species richness of various taxonomic groups across multiple scales. They found the effects of landscape structure expressed in terms of landscape metrics on species richness to strongly depend on the spatial scale of the analysis.

Finally, spatial scale also plays a role in the analysis of environmental conditions. According to SIEFERT et al. (2012) both the extent and grain size may influence the amount of variance observed in a given environmental factor. Due to the spatial au-

\footnotetext{
${ }^{10} \mathrm{cp}$. subsection 2.2.2 for the categorisation of DLM data applied in the present study
}

${ }^{11}$ i. e. the size of the smallest unit of observation, (PALMER \& White, 1994; Turner, 2005) 
tocorrelation observed in most environmental variables, their variance is supposed to increase when the extent of the study area is enlarged (WIENS, 1989; SIEFERT et al., 2012). However, the size of this effect largely varies between different environmental variables. SIEFERT et al. (2012) distinguish coarse- and fine-grained variables. The former include e. g. climate data which vary across broader scales, the latter are represented by e.g. edaphic variables that may show a large variation even within a small area.

The aforementioned examples clearly illustrate the importance of scale. However, the knowledge of this scale-dependence of landscape-level approaches can only rarely be included in research as the spatial extent and grain of an analysis are usually determined by the availability of the base data.

\subsubsection{Bias due to data format}

"Yes, raster is faster, but raster is vaster, and vector just seems more corrector."

C. Dana Tomlin

For environmental assessments using GIS, both vector and raster data are commonly used (WADE et al., 2003). Usually, vector data are referred to as allowing high levels of cartographic accuracy ${ }^{12}$ (WYATT \& RALPHS, 2003). In contrast, raster data are supposed to be less precise than vector data as it is difficult to "represent objects with a sufficient level of cartographic precision using raster methods, since the resolution of the raster grid needs to be prohibitively high to capture an equivalent level of detail" (WyatT \& RAlPhs, 2003, p. 49). Nevertheless, raster data are often used in all kinds of studies because in many cases they represent the only data source available and are faster to process (LANG \& BlASCHKE, 2007). For the present thesis, several data sources were available in raster format (e.g. DEM and climate data, see Table 2.1). Furthermore, some vector data (DLM) had to be converted to raster format as the software used for the subsequent analysis (Fragstats 3.3, McGarigal \& CUsHMAN, 2002) was limited to this type of data. In transforming the data, an additional bias is introduced because a balance between resolution and file size has to be found: If pixel size is too large, the data set will suffer from cartographic imprecision, whilst a very high resolution will generate a file too large for computation (WYATT \& RALPHS, 2003). In the present thesis the resolution of the transformed data was set to a pixel size of $25 \mathrm{~m}^{2}$. This value was chosen because of hardware limitations (cp. subsection 2.2.5). However, for the analyses conducted in context of this thesis this resolution should be sufficient to capture the overall characteristics of the landscape.

However, the pixel size of a raster not only affects the precision of the data set but can also directly influence the calculation of landscape metrics (McGARIGAL \& Cushman, 2002; NeEl et al., 2004). Indices that include the complexity of patches are particularly affected because of the step-wise structure of the patch margins that

\footnotetext{
${ }^{12}$ However, this attribute still depends on the accuracy of data acquisition.
} 
results in the overestimation of patch complexity and edge length (LEITÃo et al., 2006). In the present study this is especially true for the Total Edge as well as the Shape Index utilised in chapter 6 . The latter suffers from one further limitation: Because of the raster format the simplest shape is set to be a square even though in reality circular patches have the smallest perimeter-area relationship and thus experience fewer edge effects.

\subsubsection{Bias in species records}

In species record data the main problem is the reliability of these data (HAEUPLER, 2000; Honnay et al., 2003; DieKmann et al., 2008). Frequently, data on species occurrences are incomplete because some areas are better inventoried than others (BARTHLOTT et al., 1996). This problem apparently may provoke misleading results. Thus, it is highly important to check data on species records prior to subsequent analyses. The database utilised in the present thesis was carefully checked for its quality by the responsible authority (SCHACHERER, 2001), so that the bias related to this particular source of error should be of minor concern.

\subsection{Concluding remarks and outlook}

Although landscape-level approaches suffer from the "dilemma of indicating complexity with simple measures" (Duelli \& OBRist, 2003, p. 95) and are subject to several limitations (cp. section 7.3), they are a powerful tool to disentangle the underlying causes of species richness patterns. As many, if not most, of the processes affecting biodiversity take place at the landscape level obviously they should also be measured at this scale (McGarigal \& CUSHMAN, 2002; FAHrig, 2003). However, experimental approaches on such a large scale are rarely possible (WiLlig et al., 2003; Gotelli et al., 2009), thus statistical approaches making use of existing data sources represent the only feasible method to approach this complex topic.

By applying landscape-level approaches, the present thesis showed that patterns of vascular plant species richness in Lower Saxony and Bremen are largely influenced by anthropogenically-induced land use patterns. However, also the importance of the abiotic environment in shaping species richness patterns was revealed. All analyses were conducted using the data base of the vascular plant survey of Lower Saxony that - due to its high quality and comprehensiveness - in this context provided a solid basis for these assessments.

As already highlighted in the key findings, the individual results obtained from the four studies each contribute to landscape and systematic conservation planning by facilitating the identification and preselection of landscape units of high conservation value and concern. However, by merging the knowledge derived from the studies, an even stronger comprehensive picture of the conservation value of the landscape units can be obtained. In doing so, landscape units of high conservation value, e. g. those 
hosting a large number of species but have experienced a low degree of landscape modification and fragmentation, can be identified. In contrast, landscape units of high conservation concern that are species-rich despite being under high pressure from human land use, can be located. It is then possible to assess the protection status of these located landscape units by using the methodology applied in chapter 4 and determine if further conservation measures ought to be taken.

Arising from the discussed limitations and uncertainties connected to landscape-level approaches (cp. subsection 7.3), many open research questions remain that should be examined in more detail in further studies. As the transferability of the methodologies applied in this thesis is high (cp. section 7.2), it is possible to conduct analogue studies that will further increase the understanding of the factors influencing species richness patterns at the landscape scale. The most important and interesting research questions that should be addressed by future research approaches are:

1. How do the results change if the analyses are conducted at different spatial scales (changing grain size, changing spatial extent)?

2. Are the results also observed in other regions and particularly in more natural landscapes with shorter history of cultivation?

3. How do the results change if not species richness but species composition is used as the response variable in the analyses?

Progress in terms of the first research question could be made by using point-based species records, as these can be summarised into grid data of varying resolutions. For the study region of the present thesis such data could become available in the near future as the third survey period of the vascular plant survey of Lower Saxony (2004-2014) is currently assessing point-based species occurrence data.

The second research question could be addressed by transferring the methodologies of this thesis to other federal states of Germany or even other Central European countries. To test if the results are reproducible even in landscapes with a less extensive history of cultivation, a transfer to areas outside of Central Europe, e. g. to one of the northern European countries is needed. The 'Kasviatlas' of Finland (see Table 7.1) in this context could form a suitable basis for such a continuative analysis.

Finally, the third question would be particularly interesting to examine as several researchers (HILlEBRAND et al., 2008; CROWDER et al., 2010; TSCHARNTKE et al., 2012) have suggested that changes in species composition and evenness might have even larger effects on an ecosystem than changes in species richness. However, grid-based data at higher resolution than that utilised in the present thesis would probably need to be used for such an analysis as preliminary analyses conducted in context of this thesis did not return interpretable results.

By approaching these research questions, further progress will be made in the disentanglement of the "high real-world complexity shaping [species richness] patterns" (TsCHARNTKE et al., 2012, p. 678). 


\section{References}

Alabama Herbarium Consortium (2014): Alabama Plant Atlas. URL http:/ /www. floraofalabama.org.

Andelman, S. J. \& Willig, M. R. (2003): Present patterns and future prospects for biodiversity in the Western Hemisphere. Ecology Letters 6: 818-824.

Angelstam, P. (2006): Maintaining cultural and natural biodiversity in Europe's economic centre and periphery. In: Agnoletti, M. (ed.) The conservation of cultural landscapes, CABI, Wallingford. pp. 125-143.

Bailey, D.; Herzog, F.; Augenstein, I.; Aviron, S.; Billeter, R.; Szerencsits, E. \& BAUDRY, J. (2007): Thematic resolution matters: Indicators of landscape pattern for European agro-ecosystems. Ecological Indicators 7: 692-709.

Barthlott, W.; Lauer, W. \& Placke, A. (1996): Global distribution of species diversity in vascular plants: Towards a world map of phytodiversity. Erdkunde 50: 317-327.

Buyantuyev, A. \& Wu, J. (2007): Effects of thematic resolution on landscape pattern analysis. Landscape Ecology 22: 7-13.

Crowder, D. W.; Northfield, T. D.; Strand, M. R. \& Snyder, W. E. (2010): Organic agriculture promotes evenness and natural pest control. Nature 466: 109-112.

Culmsee, H.; Schmidt, M.; Schmiedel, I.; Schacherer, A.; Meyer, P. \& Leuschner, C. (2014): Predicting the distribution of forest habitat types using indicator species to facilitate systematic conservation planning. Ecological Indicators 37: 131-144.

Deguise, I. E. \& Kerr, J. T. (2006): Protected areas and prospects for endangered species conservation in Canada. Conservation Biology 20: 48-55.

Diekmann, M.; Dupré, C.; Kolb, A. \& Metzing, D. (2008): Forest vascular plants as indicators of plant species richness: A data analysis of a flora atlas from northwestern Germany. Plant Biosystems 142: 584-593.

Duelli, P. \& OвRist, M. K. (2003): Biodiversity indicators: the choice of values and measures. Agriculture, Ecosystems \& Environment 98: 87-98.

EEA (European Environment Agency) (2004): High nature value farmland: Characteristics, trends, and policy challenges, EEA Report, vol. 1. EEA, Copenhagen. 26 pp.

EEA (European Environment Agency) (2013): Corine Land Cover 2006 raster data: Version $17(12 / 2013)$ - Raster data on land cover for the 
CLC2006 inventory. URL http://www.eea.europa.eu/data-and-maps/data/ corine-land-cover-2006-raster-3. Retrieved 08/26/2014.

FAHRIG, L. (2003): Effects of habitat fragmentation on biodiversity. Annual Review of Ecology, Evolution, and Systematics 34: 487-515.

Finnish Museum of Natural History (2014): The database of Atlas Florae Europaeae. URL http://www.luomus.fi/en/database-atlas-florae-europaeae. Retrieved 09/24/2014.

Girvetz, E. H.; Thorne, J. H.; Berry, A. M. \& Jaeger, J. A. G. (2008): Integration of landscape fragmentation analysis into regional planning: A statewide multi-scale case study from California, USA. Landscape and Urban Planning 86: 205-218.

Gotelli, N. J.; Anderson, M. J.; Arita, H. T.; ChaO, A.; Colwell, R. K.; Connolly, S. R.; Currie, D. J.; Dunn, R. R.; Graves, G. R.; Green, J. L.; Grytnes, J.-A.; Jiang, Y.-H.; Jetz, W.; Lyons, K. S.; McCain, C. M.; Magurran, A. E.; RahbeK, C.; Rangel, T. F. L. V. B.; Soberón, J.; Webb, C. O. \& Willig, M. R. (2009): Patterns and causes of species richness: a general simulation model for macroecology. Ecology Letters 12: 873-886.

Green, R. D. \& Hartley, S. (2000): Integrating photointerpretation and GIS for vegetation mapping: Some Issues of Error. In: Alexander, R. W. \& Millington, C. A. (eds.) Vegetation mapping: From Patch to Planet, John Wiley, Chichester. pp. 103-134.

Haeupler, H. (1974): Statistische Auswertung von Punktrasterkarten der Gefäßpflanzenflora Süd-Niedersachsens. Scripta Geobotanica 8: 1-141.

Haeupler, H. (2000): Die ungleiche Verteilung der Artenvielfalt innerhalb Deutschlands - Eine Antwort. Floristische Rundbriefe 34: 17-24.

Haeupler, H.; Jagel. A. \& Schumacher, W. (2003): Verbreitungsatlas der Farn- und Blütenpflanzen in Nordrhein-Westfalen. Landesamt für Ökologie, Bodenordnung und Forsten NRW, Recklinghausen. 616 pp.

Haeupler, H. \& Vogel, A. (1999): Plant diversity in Germany: a second review. Acta Botanica Fennica 162: 55-59.

HaRdtKe, H.-J. \& IhL, A. (2000): Atlas der Farn- und Samenpflanzen Sachsens. Dresden. 806 pp.

He, F.; LaFrankie, J. V. \& Song, B. (2002): Scale dependence of tree abundance and richness in a tropical rain forest, Malaysia. Landscape Ecology 17: 559-568. 
Hijmans, R. J.; Cameron, S. \& Parra, J. (2013): WorldClim - Global Climate Data: Free climate data for ecological modelling and GIS. URL http://www.worldclim. org/. Retrieved 08/26/2014.

Hillebrand, H.; Bennett, D. M. \& Cadotte, M. W. (2008): Consequences of dominance: A review of evenness effects on local and regional ecosystem processes. Ecology 89: 1510-1520.

Honnay, O.; Piessens, K.; Landuyt, W. v.; Hermy, M. \& Gulinck, H. (2003): Satellite based land use and landscape complexity indices as predictors for regional plant species diversity. Landscape and Urban Planning 63: 241-250.

Jackson, S. F.; WALKeR, K. \& GAStON, K. J. (2009): Relationship between distributions of threatened plants and protected areas in Britain. Biological Conservation 142: $1515-1522$.

JAEGER, J. A. G. (2000): Landscape division, splitting index, and effective mesh size: New measures of landscape fragmentation. Landscape Ecology 15: 115-130.

Jaeger, J. A. G.; Bertiller, R.; Schwick, C.; Müller, K.; Steinmeier, C.; Ewald, K. C. \& GHAzoul, J. (2008): Implementing landscape fragmentation as an indicator in the Swiss Monitoring System of Sustainable Development (MONET). Journal of Environmental Management 88: 737-751.

Jalas, J. \& Suominen, J. (eds.) (1972-2013): Atlas Florae Europaeae: 16 volumes. The Committee for Mapping the Flora of Europe and Societas Biologica Fennica Vanamo, Helsinki.

Kallimanis, A. S.; Halley, J. M.; Vokou, D. \& Sgardelis, S. P. (2008): The scale of analysis determines the spatial pattern of woody species diversity in the Mediterranean environment. Plant Ecology 196: 143-151.

Korsch, H.; Westhus, W. \& ZÜNDORF, H.-J. (2002): Verbreitungsatlas der Farn- und Blütenpflanzen Thüringens. Weissdorn-Verlag, Jena. 419 pp.

KOWARIK, I. (1990): Some responses of flora and vegetation to urbanization in Central Europe. In: Sukopp, H.; Hejný, S. \& Kowarik, I. (eds.) Urban ecology, SPB Academic Publishing, Den Hague, Netherlands. pp. 45-74.

KühN, I.; Brandl, R.; MaY, R. \& KLOTZ, S. (2003): Plant distribution patterns in Germany - Will aliens match natives? Feddes Repertorium 114: 559-573.

Landuyt, W. v.; Vanhecke, L. \& Brosens, D. (2012): Florabank1: a grid-based database on vascular plant distribution in the northern part of Belgium (Flanders and the Brussels Capital region). PhytoKeys 12: 59-67. 
LANG, S. \& BlaschKe, T. (2007): Landschaftsanalyse mit GIS. Ulmer, Stuttgart. 404 pp.

Leitão, A. B.; Miller, J. N.; Ahern, J. \& McGarigal, K. (2006): Measuring landscapes: A planner's handbook. Island Press, Washington. 245 pp.

LIPPE, M. v. D. \& KowARIK, I. (2008): Do cities export biodiversity? Traffic as dispersal vector across urban-rural gradients. Diversity and Distributions 14: 18-25.

Machado, A. (2004): An index of naturalness. Journal for Nature Conservation 12: 95-110.

Manakos, I. \& Braun, M. (2014): Land use and land cover mapping in Europe: Practices \& trends, vol. 18. Springer, Dordrecht. 441 pp.

McGarigal, K. \& Cushman, S. A. (2002): Comparative evaluation of experimental approaches to the study of habitat fragmentation effects. Ecological Applications 12: 335-345.

Neel, M. C.; McGarigal, K. \& Cushman, S. A. (2004): Behavior of class-level landscape metrics across gradients of class aggregation and area. Landscape Ecology 19: $435-455$.

NetPhyD \& BFN (2013): Verbreitungsatlas der Farn- und Blütenpflanzen Deutschlands. Landwirtschaftsverlag Münster, Münster. 912 pp.

New York Flora Association (2014): New York Flora Atlas. URL http://www. newyork.plantatlas.usf.edu/.

Palmer, M. W. \& White, P. S. (1994): Scale dependence and the species-area relationship. American Naturalist 144: 717-740.

Rodrigues, A. S. L.; Andelman, S. J.; Bakarr, M. I.; Boitani, L.; Brooks, T. M.; Cowling, R. M.; Fishrool, L. D.; Da Fonseca, G. A. B.; Gaston, K. J.; Hoffmann, M.; Long, J. S.; Marquet, P. A.; Pilgrim, J. D.; Pressey, R. L.; Schipper, J.; Sechrest, W.; Stuart, S. N.; Underhill, L. G.; Waller, R. W.; Watts, M. E. \& YAN, X. (2004): Effectiveness of the global protected area network in representing species diversity. Nature 428: 640-643.

SAURA, S. \& Martinez-Millán, J. (2001): Sensitivity of landscape pattern metrics to map spatial extent. Photogrammetric Engineering \& Remote Sensing 67: 1027-1036.

Schacherer, A. (2001): Das Niedersächsische Pflanzenarten-Erfassungsprogramm. Informationsdienst Naturschutz Niedersachsen 21 (5/01 Supplement Pflanzen): 1-20.

Schetter, T. A.; Walters, T. L. \& Root, K. V. (2013): A multi-scale spatial analysis of native and exotic plant species richness within a mixed-disturbance oak savanna landscape. Environmental Management 52: 581-594. 
Schindler, S.; Wehrden, H. v.; Poirazidis, K.; WrbKa, T. \& Kati, V. (2013): Multiscale performance of landscape metrics as indicators of species richness of plants, insects and vertebrates. Ecological Indicators 31: 41-48.

Schmiedel, I.; Bergmeier, E. \& Culmsee, H. (submitted): Plant species richness patterns along a gradient of landscape modification intensity in Lower Saxony, Germany .

Schmiedel, I. \& Culmsee, H. (submitted): The influence of landscape fragmentation, expressed by the 'effective mesh size index', on regional patterns of vascular plant species richness in Lower Saxony, Germany .

Schmiedel, I.; Schacherer, A.; Hauck, M.; Schmidt, M. \& Culmsee, H. (2011): Verbreitungsmuster der Farn- und Blütenpflanzen in Niedersachsen und Bremen unter Berücksichtigung ihres Einbürgerungsstatus und ihrer Gefährdungssituation. Tuexenia 31: 211-226.

Schmiedel, I.; Schmidt, M.; Schacherer, A. \& Culmsee, H. (2013): Die Effektivität von Schutzgebieten für die Erhaltung seltener und gefährdeter Gefäßpflanzenarten: Eine Untersuchung im niedersächsischen Tiefland. Naturschutz und Landschaftsplanung 45: 45-52.

Scott, J. M.; Davis, F. W.; McGhie, R. G.; Wright, R. G.; Groves, C. \& Estes, J. (2001): Nature reserved: Do they capture the full range of America's biological diversity? Ecological Applications 11: 999-1007.

Siefert, A.; Ravenscroft, C.; Althoff, D.; Alvarez-Yépiz, J. C.; Carter, B. E.; Glennon, K. L.; Heberling, J. M.; Jo, I. S.; Pontes, A.; Sauer, A.; Willis, A.; Fridley, J. D. \& AcostA, A. (2012): Scale dependence of vegetation-environment relationships: a meta-analysis of multivariate data. Journal of Vegetation Science 23: 942-951.

The Calflora Database (2014): Calflora: Information on wild California plants for education, research and conservation. URL http://www.calflora.org/.

TscharntKe, T.; Tylianakis, J. M.; Rand, T. A.; Didham, R. K.; Fahrig, L.; Batáry, P.; Bengtsson, J.; Clough, Y.; Crist, T. O.; Dormann, C. F.; Ewers, R. M.; Fründ, J.; Holt, R. D.; Holzschun, A.; Klein, A. M.; Kleijn, D.; Kremen, C.; Landis, D. A.; Laurance, W.; Lindenmayer, D.; Scherber, C.; Sodhi, N.; Steffan-Dewenter, I.; Thies, C.; van der Putten, W. H \& Westphal, C. (2012): Landscape moderation of biodiversity patterns and processes - eight hypotheses. Biological reviews of the Cambridge Philosophical Society 87: 661-685.

Turner, M. G. (2005): Landscape ecology: What is the state of the science? Annual Review of Ecology, Evolution, and Systematics 36: 319-344. 
Turner, M. G.; O’Neill, R. V.; Gardner, R. H. \& Milne, B. T. (1989): Effects of changing spatial scale on the analysis of landscape pattern. Landscape Ecology 3: 153-162.

University of Florida, Institute for Systematic Botany (2014): Atlas of Florida Vascular Plants. URL http://www.florida.plantatlas.usf.edu/.

Wade, T. G.; Wickham, J. D.; Nash, M. S.; Neale, A. C.; Rittters, K. H. \& Jones, K. B. (2003): A comparison of vector and raster GIS methods for calculating landscape metrics used in environmental assessments. Photogrammetric Engineering $\mathcal{E}$ Remote Sensing 69: 1399-1405.

WALz, U. \& STEIN, C. (2014): Indicators of hemeroby for the monitoring of landscapes in Germany. Journal for Nature Conservation 22: 279-289.

WaniA, A.; KüHN, I. \& KLOTZ, S. (2006): Plant richness patterns in agricultural and urban landscapes in Central Germany - spatial gradients of species richness. Landscape and Urban Planning 75: 97-110.

WIENS, J. A. (1989): Spatial scaling in ecology. Functional Ecology 3: 385-397.

Willig, M. R.; Kaufman, D. M. \& Stevens, R. D. (2003): Latitudinal gradients of biodiversity: Pattern, process, scale, and synthesis. Annual Review of Ecology, Evolution, and Systematics 34: 273-309.

Wu, J. (2004): Effects of changing scale on landscape pattern analysis: scaling relations. Landscape Ecology 19: 125-138.

Wyatt, P. \& Ralphs, M. (2003): GIS in land and property management. Spon Press, London and New York. 390 pp.

YANG, W.; MA, K. \& KREFT, H. (2013): Geographical sampling bias in a large distributional database and its effects on species richness-environment models. Journal of Biogeography 40: 1415-1426.

ZaJAC, A. \& ZAJAC, M. (2001): Distribution atlas of vascular plants in Poland. Kraków. $714 \mathrm{pp}$. 



\section{Acknowledgments}

My special thanks go to my supervisor Dr. Heike Culmsee for giving me the opportunity to conduct my Ph.D. studies in the framework of her project "Identification of indicator species groups of grassland and forest habitats for biodiversity monitoring and evaluation" and for her support during that time. Furthermore, I would like to thank Prof. Erwin Bergmeier for his help all along the way and for giving me the opportunity to work in his department. I would like to thank him and Prof. C. Leuschner as well as Prof. H. Behling, Prof. S. Bögeholz, Prof. R. Bürger-Arndt and Prof. M. Hauck for being part of my Ph.D. and defence committee.

Since the thesis was embedded in the above mentioned project funded by the Deutsche Bundesstiftung Umwelt (DBU), I would also like to thank the DBU for its financial support. The project was undertaken in co-operation with the NLWKN, namely Dr. Annemarie Schacherer and Michael Hauck as well as the NW-FVA, namely Dr. Marcus Schmidt - it was a pleasure to collaborate with you! Furthermore, I am really grateful to all volunteers working for the vascular plant survey of Lower Saxony - without the participation of all these people such an extensive dataset would not have come to life!

My most heartfelt thanks go to all my colleagues at the department of 'Vegetation \& Phytodiversity Analysis' for all the lively discussions during coffee breaks and Mensa and the nice working atmosphere - I always felt really comfortable in your company! Special thanks at this point to my friend and room-mate Florian Goedecke for our nice, busy and often funny times in the office and in the field and all his help!

I would like to particularly thank Laura Sutcliffe for her proofreading of large parts of this thesis and for the revision of many articles and other small texts over the past years. In this context special thanks also to Florian Goedecke, Helen Rosenkranz and Christoph Windolph for helping with the correction of the thesis. Christoph, thank you also for your 'technical' support - without your help I would often have been lost in the $\mathrm{LT}_{\mathrm{E}} \mathrm{X}$ jungle...

Turning to the 'non-scientific side' of my life I especially want to thank my parents Bernd and Christine Schmiedel, my partner Christoph Windolph as well as my grandfather Helmut Arhold for always being there for me, supporting me, raising my spirit whenever needed and trusting in me - without all your help and encouragement I would not have finished the thesis!

Last but not least I also want to thank my 'dear old' friends Helen, Dorothée, Miriam, Jovana and Torsten with whom I spend so much nice time - thank you for being there for me in all these years! 



\section{Academic CV}

\section{Inga Schmiedel}

born on $15^{\text {th }}$ of January 1984 in Göttingen, Germany

\section{Education}

2009 - 2014: $\quad$ PhD studies, Dpt. Vegetation \& Phytodiversity Analysis, University of Göttingen, Germany.

2012 - 2013: Completion of a certificate programme on teaching at the center for 'Teaching and Learning in Higher Education', University of Göttingen/TU Clausthal.

2006 - 2008: $\quad$ Advanced studies of biology, University of Göttingen.

Main interests: Vegetation and plant ecology, nature conservation and geography. Topic of the diploma thesis: 'Analyse der Landschaftseinnischung von Kalkmagerrasen am Mühlenberg bei Barterode (Landkreis Göttingen) auf Grundlage einer Biotoptypenkartierung'.

2005 - 2006: $\quad$ Advanced studies, University of Umeå, Sweden.

2003 - 2005: Basic studies of biology, University of Göttingen.

2003

Qualification for university entrance (Abitur), Gymnasium Uslar, Germany.

\section{Work experience}

2010 - 2014: Research and teaching assistant, Dpt. Vegetation \& Phytodiversity Analysis, University of Göttingen.

2010 - 2012: $\quad$ Mapping of Natura 2000 habitat types on a military area in Saxony-Anhalt (Co. GISCON, Braunschweig).

2009-2010: Research assistant in the project 'Identification of indicator species groups of grassland and forest habitats for biodiversity monitoring and evaluation', Dpt. Vegetation \& Phytodiversity Analysis, University of Göttingen.

2009: $\quad$ Freelance work (biotope mapping, DBU project 'Nature conservation and cultural conservation in a castle mountain landscape in Thuringia, Germany').

2008, 2009: Teaching assistant (GIS course), University of Göttingen.

2006 - 2008: Student assistant at the AvH-Institute for Plant Sciences, University of Göttingen 



\section{List of publications}

\section{Peer reviewed journal publications}

Schmidt, M.; Mölder, A.; Schönfelder, E.; Engel, F.; Schmiedel, I. \& Culmsee, H. (2014): Determining ancient woodland indicator plants for practical use: A new approach developed in northwest Germany. Forest Ecology and Management 330: 228-239.

Culmsee, H.; Schmidt, M.; Schmiedel, I.; Schacherer, A.; Meyer, P. \& Leuschner, C. (2014): Predicting the distribution of forest habitat types using indicator species to facilitate systematic conservation planning. Ecological Indicators 37: $131-14$

Schmiedel, I.; Schmidt, M.; Schacherer, A. \& Culmsee, H. (2013): Die Effektivität von Schutzgebieten für die Erhaltung seltener und gefährdeter Gefäßpflanzenarten. Eine Untersuchung im niedersächsischen Tiefland. [The effectiveness of protection areas for the conservation of rare and endangered vascular plant species. An investigation in the lowlands of Lower Saxony, Germany.]. Naturschutz und Landschaftsplanung 45: 45-52.

Schmiedel, I.; Schacherer, A.; Hauck, M.; Schmidt, M. \& Culmsee, H. (2011): Verbreitungsmuster der Farn- und Blütenpflanzen in Niedersachsen und Bremen unter Berücksichtigung ihres Einbürgerungsstatus und ihrer Gefährdungssituation [Distribution patterns of vascular plant taxa in the federal states of Lower Saxony and Bremen, Germany, with respect to their naturalisation and threat status.]. Tuexenia 31: 211-226.

\section{Submitted journal publications}

Schmiedel, I.; Bergmeier, E. \& Culmsee, H.: Plant species richness patterns along a gradient of landscape modification intensity in Lower Saxony, Germany. Submitted.

Schmiedel, I. \& Culmsee, H.: The influence of landscape fragmentation, expressed by the 'Effective Mesh Size Index', on regional patterns of vascular plant species richness in Lower Saxony, Germany. Submitted. 


\section{Conference proceedings}

Schmiedel, I. \& Culmsee, H. (2014): The 'effective mesh size index' as a predictor for regional patterns of vascular plant species richness. 44. Annual Meeting of the Ecological Society of Germany, Austria and Switzerland (GfÖ), $8^{\text {th }}-12^{\text {th }}$ September 2014. Verhandlungen der Gesellschaft für Ökologie 44, p. 60.

Schmiedel, I.; Schmidt, M.; Schacherer, A. \& Culmsee, H. (2012): The effectiveness of protected areas for the conservation of rare and endangered vascular plant species in the Lowlands of Lower Saxony, Germany. 42. Annual Meeting of the Ecological Society of Germany, Austria and Switzerland (GfÖ), $10^{\text {th }}-14^{\text {th }}$ September 2012. Verhandlungen der Gesellschaft für Ökologie 42, p. 76.

Schmiedel, I. \& Culmsee, H. (2009): Landscape fragmentation and its effect on vascular plant species richness in Lower Saxony. 39. Annual Meeting of the Ecological Society of Germany, Austria and Switzerland (GfÖ), $14^{\text {th }}-18^{\text {th }}$ September 2009. Bayreuther Forum für Ökologie 115, p. 233.

\section{Other publications}

Siegesmund, S.; Hopf, U.; Stück, H.; Beutler, G.; Culmsee, H.; Hoppert, M.; Reeh, T.; Schmiedel, I.; Schubert, J.; Schwienheer, J. \& Wieland, T. (2013): Die Kulturlandschaft der Drei-Gleichen-Region in Thüringen. In: Siegesmund, S. \& Snethlage, R. (eds.), Naturstein in der Kulturlandschaft. Mitteldeutscher Verlag, Halle (Saale). pp. 334-347

Schmidt, M.; Culmsee, H.; Boch, S.; Heinken, T.; Müller, J. \& Schmiedel, I. (2011): Anwendungsmöglichkeiten von Waldartenlisten für Gefäßpflanzen, Moose und Flechten. - BfN-Skripten 299: 25-45.

Culmsee, H.; Herrling, J.; Schmiedel, I.; Schwienheer, J. \& Wolf, D. (2010): Die Vegetation der Drei Gleichen im Spiegel der historischen Nutzung und ihre Bedeutung für den Naturschutz. In: Siegesmund, S. \& Hoppert, M. (eds.), Die Drei Gleichen - Baudenkmäler und Naturraum. Edition Leipzig, Leipzig. pp. 202-222. 


\section{Appendix A}

Table A.1: Bio-geographical regions and natural landscape units of Lower Saxony. Basis: PiLgRIM \& FrANKe (1993).

\begin{tabular}{|c|c|c|}
\hline Bio-geographical regions & Natural landscape unit & No. \\
\hline Coast & & \\
\hline Watten und Marschen & $\begin{array}{l}\text { Emsmarschen } \\
\text { Harburger Elbmarschen } \\
\text { Wesermarschen }\end{array}$ & $\begin{array}{l}610 \\
670 \\
612\end{array}$ \\
\hline
\end{tabular}

Lowlands

2 Ostfriesisch-Oldenburgische Geest

Aschendorfer Emstal

604

Bourtanger Moor

605

Hunte-Leda-Moorniederung 600

Oldenburger Geest $\quad 603$

Ostfriesische Geest $\quad 602$

Ostfriesische Zentralmoore $\quad 601$

3 Stader Geest $\quad$ Achim-Verdener Geest 630

Hamme-Oste-Niederung 632

Wümmeniederung 631

Wesermünder Geest $\quad 633$

Zevener Geest 634

4 Ems-Hunte-Geest und Bersenbrücker Land 585

Dümmer-Geestniederung Cloppenburger Geest 593

Delmenhorster Geest $\quad 595$

Diepholzer Moorniederung $\quad 584$

Lingener Land 586

Nordhorn-Bentheimer Sandgebiet $\quad 580$

Ostmünsterland $\quad 540$

Plantlünner Sandebene 581

Rahden-Diepenauer Geest $\quad 582$

Sögeler Geest (Hümmling) 592

Syker Geest $\quad 594$

Westmünsterland 544

5 Lüneburger Heide und Hohe Heide 640

Wendland Jeetzel-Dumme-Lehmplatte und Arendseer Platte $\quad 861$

Lüchower Niederung $\quad 860$

Luheheide 644

Ostheide 642 
Table A.1 - Continued from previous page

\begin{tabular}{lll}
\hline Bio-geographical regions & Natural landscape unit & No. \\
\hline \multirow{2}{*}{ Weser-Aller-Flachland } & Südheide & 641 \\
& Uelzener und Bevenser Becken & 643 \\
& Untere Mittelelbe-Niederung & 876 \\
& Westaltmärkisches Waldhügelland & 865 \\
& Burgdorf-Peiner Geestplatten & 623 \\
& Drömling & 625 \\
& Hannoversche Moorgeest & 622 \\
& Loccumer Geest & 628 \\
& Mittelweser & 583 \\
& Mittleres Wesertal & 583 \\
& Obere Allerniederung & 626 \\
& Ostbraunschweigisches Flachland & 624 \\
& Thedinghäuser Vorgeest & 621 \\
& Untere Aller-Talsandebene & 627 \\
& Verdener Wesertal & 620
\end{tabular}

\section{Uplands}

7 Börden

Bückebergvorland 522

Braunschweig-Hildesheimer Lössbörde $\quad 520$

Calenberger Lössbörde 521

Großes Bruch 511

Nördliches Harzvorland, Westteil 510

Ostbraunschweigisches Hügelland 512

8.1 Osnabrücker Hügelland Östliches Wiehengebirge 532

Lübbecker Lössland 533

Osnabrücker Hügelland 535

Osnabrücker Osning 534

Ravensberger Hügelland 531

Westliches Wiehengebirge 536

8.2 Weser- und Leinebergland Alfelder Bergland (mit Ith und Hils) 377

Eichsfelder Becken (Goldene Mark) 374

Fulda-Werra-Bergland 357

Göttingen-Northeimer Wald 373

Holzmindener Wesertal $\quad 367$

Innerstebergland $\quad 379$

Kalenberger Bergland $\quad 378$

Leine-Ilme-Senke $\quad 372$

Lipper Bergland $\quad 364$

Oberwälder Land 361

Pyrmonter Bergland $\quad 365$

Rinteln-Hamelner Weserland $\quad 366$

Südharzer Zechsteingürtel $\quad 485$

Südliches Harzvorland 486

Südwestliches Harzvorland 376 
Table A.1 - Continued from previous page

\begin{tabular}{lll}
\hline Bio-geographical regions & Natural landscape unit & No. \\
\hline \multirow{6}{*}{ Harz } & Solling, Bramwald und Reinhardswald & 370 \\
& Sollingvorland & 371 \\
& Unteres Werratal & 358 \\
& Weserengtal von Bodenwerder & 368 \\
& Westhessische Senke & 343 \\
& & 381 \\
& Hochharz & 380 \\
& Oberharz & 382
\end{tabular}

Table A.2: List of vascular plant species of the data base of the vascular plant survey of Lower Saxony. The table lists only those species for which occurrences are reported. Extinct species are not listed. Species were aggregated on the highest taxonomical level found in the data base, i. e. subspecies were assigned to the respective species and species were assigned to the respective aggregate if the latter was listed in the data base. 'Edge' denotes that the species is present in the grid cells placed at the border of the state only (cp. section 2.1; Natural. = naturalisation (nat $=$ native taxa, $\operatorname{arc}=$ archaeophytes, neo $=$ neophytes); RL NB $=$ Red List status assigned by GARVE (2004), categories 1, 2, 3, G and R denote threatened taxa, category ' $\mathrm{V}$ ' denotes prewarned taxa, '*' denotes non-threatened taxa (cp. Table 2.2); EIV N1-3/N7-9 = Plant species of nutrient-poor/nutrient-rich habitats (Ellenberg Indicator Values for nutrients 1-3 and 7-9; $\mathrm{BFN}, 2013$ ); Urban = Plant species of urban habitats (urbanity indicator 4 and 5; KLOTZ et al., 2002); Forest $=$ Herbaceous and shrubby plant species linked to forest habitats (forest affinity categories 1.1 and 1.2; SснмIDT et al., 2011); Code = Code used in the data base of the NLWKN (1982-2003); Incl. = Codes of (sub-)species assigned to the particular taxon, see Table A.3 for full names of (sub-)species.

\begin{tabular}{|c|c|c|c|c|c|c|c|c|c|}
\hline 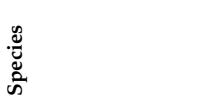 & 总 & $\begin{array}{l}\text { त्ञ } \\
\frac{\tilde{\Xi}}{\tilde{Z}}\end{array}$ & $\begin{array}{l}\underset{Z}{Z} \\
\underset{\sim}{z}\end{array}$ & $\begin{array}{l}\stackrel{p}{Z} \\
z \\
z\end{array}$ & $\begin{array}{l}\text { ì } \\
\text { 之 } \\
\vec{z}\end{array}$ & 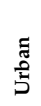 & $\begin{array}{l}\overrightarrow{\mathbb{D}} \\
\stackrel{0}{0} \\
\end{array}$ & $\frac{\pi}{8}$ & 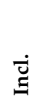 \\
\hline Acer campestre & & nat & * & & & & & $\mathrm{cab}$ & \\
\hline Acer platanoides & & nat & * & & & & & $\mathrm{cad}$ & \\
\hline Acer pseudoplatanus & & nat & * & & $\mathrm{x}$ & & & cae & \\
\hline Achillea collina & & nat & $\mathrm{R}$ & $\mathrm{x}$ & & & & caf & \\
\hline Achillea millefolium agg. & & nat & * & & & & & eab & cag \\
\hline Achillea pannonica & & nat & $\mathrm{R}$ & $\mathrm{x}$ & & & & aaa & \\
\hline Achillea ptarmica & & nat & * & $\mathrm{x}$ & & & & $\mathrm{cah}$ & \\
\hline Acinos arvensis & & nat & $\mathrm{V}$ & $\mathrm{x}$ & & & & $a a b$ & \\
\hline Aconitum lycoctonum & & nat & 3 & & $\mathrm{x}$ & & & aac & \\
\hline Acorus calamus & & neo & * & & $\mathrm{x}$ & & & cai & \\
\hline Actaea spicata & & nat & * & & $\mathrm{x}$ & & $\mathrm{x}$ & aad & \\
\hline Adonis aestivalis & & $\operatorname{arc}$ & 2 & $\mathrm{x}$ & & & & aae & \\
\hline Adonis vernalis & & nat & 2 & $\mathrm{x}$ & & & & aag & \\
\hline Adoxa moschatellina & & nat & * & & $\mathrm{x}$ & & $\mathrm{x}$ & caj & \\
\hline Aegopodium podagraria & & nat & * & & $\mathrm{x}$ & & & $\mathrm{cal}$ & \\
\hline Aethusa cynapium & & arc & * & & & & & eac & can \\
\hline Agrimonia eupatoria & & nat & * & & & & & $\mathrm{aah}$ & \\
\hline Agrimonia procera & & nat & * & & & & & aai & \\
\hline Agrostis canina & & nat & * & $\mathrm{x}$ & & & & cap & \\
\hline Agrostis capillaris & & nat & * & & & & & $\mathrm{caq}$ & \\
\hline Agrostis stolonifera agg. & & nat & * & & & & & ead & cas \\
\hline Agrostis vinealis & & nat & * & $\mathrm{x}$ & & & & cau & \\
\hline Aira caryophyllea & & nat & $\mathrm{V}$ & $x$ & & & & aal & \\
\hline Aira praecox & & nat & * & $\mathrm{x}$ & & & & aam & \\
\hline Ajuga genevensis & & nat & 3 & $\mathrm{x}$ & & & & aao & \\
\hline
\end{tabular}


Table A.2 - Continued from previous page

\begin{tabular}{|c|c|c|c|c|c|c|c|c|c|}
\hline $\begin{array}{l}\mathscr{\Xi} \\
\text { Dू } \\
\text { के }\end{array}$ & $\underset{j}{\stackrel{0}{0}}$ & 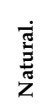 & $\begin{array}{l}\vec{Z} \\
\vec{z}\end{array}$ & $\begin{array}{l}\text { ? } \\
\mathbf{z} \\
z\end{array}$ & $\begin{array}{l}\text { à } \\
\mathbf{z} \\
\text { 嵓 }\end{array}$ & 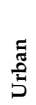 & 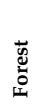 & రั & 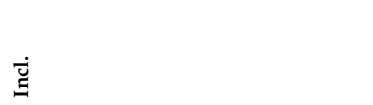 \\
\hline Ajuga reptans & & nat & * & & & & & $\operatorname{cax}$ & \\
\hline Alchemilla glaucescens & & nat & 3 & $\mathrm{x}$ & & & & aap & \\
\hline Alchemilla gramineum & $\mathrm{x}$ & nat & 1 & & & & & aas & \\
\hline Alchemilla plicata & $\mathrm{x}$ & nat & 2 & & & & & 223 & \\
\hline Alchemilla vulgaris agg. & & nat & * & & & & & aaq & cay, caz, cba, cbb,cbc, cbd, cbe, cbf, cbg \\
\hline Alisma lanceolatum & & nat & * & & & & & aat & \\
\hline Alisma plantago-aquatica agg. & & nat & * & & & & & eae & $\mathrm{cbh}$ \\
\hline Alliaria petiolata & & nat & * & & $\mathrm{x}$ & & $\mathrm{x}$ & $\mathrm{cbi}$ & \\
\hline Allium angulosum & & nat & 2 & $\mathrm{x}$ & & & & aau & \\
\hline Allium carinatum ssp. carinatum & & neo & 3 & $\mathrm{x}$ & & & & aav & \\
\hline Allium oleraceum & & nat & * & & & & & cbj & \\
\hline Allium paradoxum & & neo & * & & $\mathrm{x}$ & & $\mathrm{x}$ & $\mathrm{cbk}$ & \\
\hline Allium schoenoprasum & & neo & * & $\mathrm{x}$ & & & & aax & \\
\hline Allium scorodoprasum & & nat & 3 & & $\mathrm{x}$ & & & aay & \\
\hline Allium senescens ssp. montanum & & nat & 1 & $\mathrm{x}$ & & & & aaw & \\
\hline Allium ursinum & & nat & * & & $\mathrm{x}$ & & $\mathrm{x}$ & aaz & \\
\hline Allium vineale & & nat & * & & $\mathrm{x}$ & & & $\mathrm{cbm}$ & \\
\hline Alnus glutinosa & & nat & * & & & & & $\mathrm{cbn}$ & \\
\hline Alnus incana & & neo & * & & & & & aba & \\
\hline Alopecurus aequalis & & nat & * & & $\mathrm{x}$ & & & cbo & \\
\hline Alopecurus bulbosus & & nat & 2 & & & & & $a b b$ & \\
\hline Alopecurus geniculatus & & nat & $*$ & & $\mathrm{x}$ & & & $\mathrm{cbp}$ & \\
\hline Alopecurus myosuroides & & arc & * & & & & & $\mathrm{cbq}$ & \\
\hline Alopecurus pratensis & & nat & * & & $\mathrm{x}$ & & & $\mathrm{cbr}$ & \\
\hline Althaea hirsuta & & neo & 2 & $\mathrm{x}$ & & & & $\mathrm{cbs}$ & \\
\hline Althaea officinalis & $\mathrm{x}$ & nat & 1 & & & & & $a b c$ & \\
\hline Alyssum alyssoides & & nat & 2 & $\mathrm{x}$ & & & & abd & \\
\hline Amaranthus blitum & & arc & * & & & & & 363 & cbv, cby \\
\hline Amaranthus bouchonii & & neo & * & & & & & $\mathrm{cbw}$ & \\
\hline Amaranthus powellii agg. & & neo & * & & & $\mathrm{x}$ & & eag & $\mathrm{cbz}$ \\
\hline Amaranthus retroflexus & & neo & * & & $\mathrm{x}$ & & & cca & \\
\hline Amelanchier lamarckii & & neo & * & $\mathrm{x}$ & & & & $\mathrm{ccf}$ & \\
\hline Ammophila arenaria & & nat & * & & & & & $\mathrm{cci}$ & \\
\hline Anacamptis pyramidalis & & nat & 2 & $\mathrm{x}$ & & & & abf & \\
\hline Anagallis arvensis & & $\operatorname{arc}$ & * & & & & & cck & eah \\
\hline Anagallis foemina & & nat & 2 & & & & & abg & \\
\hline Anagallis minima & & nat & 3 & $\mathrm{x}$ & & & & ahp & \\
\hline Anchusa arvensis & & $\operatorname{arc}$ & * & & & & & $\mathrm{ccm}$ & \\
\hline Anchusa officinalis & & $\operatorname{arc}$ & $\mathrm{V}$ & & & & & $\mathrm{abh}$ & \\
\hline Andromeda polifolia & & nat & 3 & $\mathrm{x}$ & & & & abi & \\
\hline Anemone nemorosa & & nat & * & & & & $\mathrm{x}$ & $\mathrm{ccn}$ & \\
\hline Anemone ranunculoides & & nat & * & & $\mathrm{x}$ & & $\mathrm{x}$ & abk & \\
\hline Anemone sylvestris & & nat & 2 & $\mathrm{x}$ & & & & abl & \\
\hline Anemone $\mathrm{x}$ seemenii & & nat & * & & & & & 242 & \\
\hline Angelica archangelica & & nat & * & & $\mathrm{x}$ & & & $\mathrm{ccp}$ & \\
\hline Angelica sylvestris & & nat & * & & & & & $\mathrm{ccq}$ & \\
\hline Antennaria dioica & & nat & 2 & $\mathrm{x}$ & & & & $\mathrm{abm}$ & \\
\hline Anthemis arvensis & & arc & $\mathrm{V}$ & & & & & $\mathrm{abn}$ & \\
\hline Anthemis cotula & & arc & $\mathrm{V}$ & & & & & abo & \\
\hline Anthemis tinctoria & & arc & * & & & & & abp & \\
\hline Anthericum liliago & & nat & 2 & $\mathrm{x}$ & & & & $\mathrm{abq}$ & \\
\hline Anthericum ramosum & & nat & 1 & $\mathrm{x}$ & & & & $a b r$ & \\
\hline Anthoxanthum aristatum & & neo & * & $\mathrm{x}$ & & & & cct & \\
\hline Anthoxanthum odoratum & & nat & * & & & & & $\mathrm{ccu}$ & \\
\hline Anthriscus caucalis & & arc & * & & & $\mathrm{x}$ & & abs & \\
\hline Anthriscus nitida & & nat & $\mathrm{R}$ & & $\mathrm{x}$ & & $\mathrm{x}$ & abt & \\
\hline Anthriscus sylvestris & & nat & * & & $x$ & & & $\mathrm{ccw}$ & \\
\hline Anthyllis vulneraria & & nat & * & $\mathrm{x}$ & & & & 391 & $\mathrm{ccx}, \mathrm{ccy}$ \\
\hline Apera spica-venti & & nat & * & & & & & $\mathrm{ccz}$ & \\
\hline Aphanes arvensis & & arc & * & & & & & cda & \\
\hline Aphanes inexspectata & & arc & * & & & & & $c d b$ & \\
\hline Apium graveolens & & nat & 3 & & $\mathrm{x}$ & & & $a b w$ & \\
\hline Apium inundatum & & nat & 2 & $\mathrm{x}$ & & & & $a b x$ & \\
\hline Apium repens & & nat & 1 & & $\mathrm{x}$ & & & aby & \\
\hline Aquilegia vulgaris & & nat & 3 & & & & $\mathrm{x}$ & abz & \\
\hline Arabidopsis thaliana & & arc & * & & & & & $\mathrm{cdc}$ & \\
\hline Arabis glabra & & nat & $\mathrm{V}$ & & & & & $\mathrm{acb}$ & \\
\hline Arabis hirsuta agg. & & nat & * & & & & & eam & \\
\hline Arctium lappa & & arc & * & & $\mathrm{x}$ & $\mathrm{x}$ & & $\mathrm{cdg}$ & \\
\hline Arctium minus & & nat & * & & $\mathrm{x}$ & $\mathrm{x}$ & & $\mathrm{cdh}$ & \\
\hline Arctium nemorosum & & nat & * & & $\mathrm{x}$ & & $\mathrm{x}$ & cdi & \\
\hline Arctium tomentosum & & arc & * & & $x$ & $\mathrm{x}$ & & cdj & \\
\hline Arctostaphylos uva-ursi & & nat & 2 & $\mathrm{x}$ & & & & acc & \\
\hline
\end{tabular}


Table A.2 - Continued from previous page

\begin{tabular}{|c|c|c|c|c|c|c|c|c|c|}
\hline 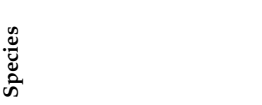 & 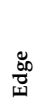 & 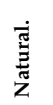 & $\begin{array}{l}\stackrel{\infty}{Z} \\
\stackrel{z}{z}\end{array}$ & $\begin{array}{l}\stackrel{p}{z} \\
\dot{z} \\
\dot{z}\end{array}$ & $\begin{array}{l}a \\
\stackrel{a}{z} \\
z\end{array}$ & 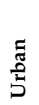 & 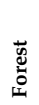 & نँ & $\begin{array}{l}\dot{\Xi} \\
\dot{\Xi}\end{array}$ \\
\hline Arenaria leptoclados & & nat & * & & & & & $\mathrm{cdk}$ & \\
\hline Arenaria serpyllifolia agg. & & nat & * & & & & & ean & cdl \\
\hline Aristolochia clematitis & & neo & 2 & & $\mathrm{x}$ & $\mathrm{x}$ & & acd & \\
\hline Armeria maritima & & nat & * & & & & & 393 & ace, acf, cdm \\
\hline Armoracia rusticana & & nat & * & & $\mathrm{x}$ & $\mathrm{x}$ & & cdn & \\
\hline Arnica montana & & nat & 2 & $\mathrm{x}$ & & & & acg & \\
\hline Arnoseris minima & & nat & 2 & $\mathrm{x}$ & & & & ach & \\
\hline Aronia x prunifolia & & neo & * & & & & & 302 & \\
\hline Arrhenatherum elatius & & nat & * & & $\mathrm{x}$ & & & cdo & \\
\hline Artemisia absinthium & & arc & * & & $\mathrm{x}$ & & & $\mathrm{cdp}$ & \\
\hline Artemisia апnиа & & neo & * & & & $\mathrm{x}$ & & $\mathrm{cdq}$ & \\
\hline Artemisia biennis & & neo & * & & & $\mathrm{x}$ & & $c d r$ & \\
\hline Artemisia campestris & & nat & $\mathrm{V}$ & $\mathrm{x}$ & & & & aci & \\
\hline Artemisia maritima & & nat & * & & $\mathrm{x}$ & & & acj & \\
\hline Artemisia vulgaris & & nat & * & & $\mathrm{x}$ & $\mathrm{x}$ & & cdt & \\
\hline Arum maculatum & & nat & * & & $\mathrm{x}$ & & $\mathrm{x}$ & acl & \\
\hline Asarum europaeum & & nat & * & & & & $\mathrm{x}$ & $\mathrm{cdu}$ & \\
\hline Asparagus officinalis & & arc & * & & & & & $c d v$ & \\
\hline Asperugo procumbens & & arc & 1 & & $\mathrm{x}$ & & & $\mathrm{acm}$ & \\
\hline Asperula cynanchica & & nat & 2 & $\mathrm{x}$ & & & & aco & \\
\hline Asplenium adiantum-nigrum & & nat & 1 & $\mathrm{x}$ & & & & acq & \\
\hline Asplenium ceterach & & nat & 1 & $\mathrm{x}$ & & & & aia & \\
\hline Asplenium ruta-muraria & & nat & * & $\mathrm{x}$ & & $\mathrm{x}$ & & acu & \\
\hline Asplenium scolopendrium & & nat & 3 & & & & $\mathrm{x}$ & ayk & \\
\hline Asplenium septentrionale & & nat & 2 & $\mathrm{x}$ & & & & acv & \\
\hline Asplenium trichomanes & & nat & * & $\hat{x}$ & & & & $\mathrm{acw}$ & 224, 206, eaq, eap \\
\hline Asplenium viride & & nat & 2 & & & & $\mathrm{x}$ & acx & \\
\hline Asplenium $\mathrm{x}$ alternifolium & & nat & $\mathrm{n} / \mathrm{a}$ & $\mathrm{x}$ & & & & acr & \\
\hline Asplenium x murbeckii & & nat & 1 & & & & & act & \\
\hline Aster amellus & & nat & 1 & $\mathrm{x}$ & & & & acy & \\
\hline Aster linosyris & & nat & 1 & $\mathrm{x}$ & & & & acz & \\
\hline Aster novi-belgii agg. & & neo & * & & & $\mathrm{x}$ & & ear & $c d w, c d x, c d y, 232,50$ \\
\hline Aster tripolium & & nat & * & & $\mathrm{x}$ & & & ada & \\
\hline Astragalus cicer & & nat & 2 & $\mathrm{x}$ & & & $\mathrm{x}$ & $\mathrm{adb}$ & \\
\hline Astragalus danicus & & nat & 1 & $\mathrm{x}$ & & & & adc & \\
\hline Astragalus glycyphyllos & & nat & * & $\mathrm{x}$ & & & $\mathrm{x}$ & $\mathrm{cdz}$ & \\
\hline Athyrium distentifolium & $\mathrm{x}$ & nat & $\mathrm{R}$ & & $\mathrm{x}$ & & & add & \\
\hline Athyrium filix-femina & & nat & * & & & & $\mathrm{x}$ & cea & \\
\hline Atriplex glabriuscula & & nat & $\mathrm{R}$ & & $\mathrm{x}$ & & & ade & \\
\hline Atriplex laciniata & $\mathrm{x}$ & nat & $\mathrm{R}$ & & $\mathrm{x}$ & & & adf & \\
\hline Atriplex littoralis & & nat & $*$ & & $\mathrm{x}$ & & & cec & \\
\hline Atriplex longipes & & nat & * & & $\mathrm{x}$ & & & ced & \\
\hline Atriplex micrantha & & neo & * & & & $\mathrm{x}$ & & cee & \\
\hline Atriplex oblongifolia & & neo & * & & & & & cef & \\
\hline Atriplex patula & & $\operatorname{arc}$ & * & & $\mathrm{x}$ & & & ceg & \\
\hline Atriplex pedunculata & & nat & 3 & & $\mathrm{x}$ & & & apo & \\
\hline Atriplex portulacoides & & nat & * & & $\mathrm{x}$ & & & ceh & \\
\hline Atriplex prostrata agg. & & nat & * & & & & & eas & cei \\
\hline Atriplex rosea & & neo & * & & $\mathrm{x}$ & $x$ & & cej & \\
\hline Atriplex sagittata & & neo & * & & $\mathrm{x}$ & & & cek & \\
\hline Atriplex tatarica & & neo & * & & & & & 13 & \\
\hline Atropa bella-donna & & nat & * & & $x$ & & $x$ & cel & \\
\hline Avena fatua & & $\operatorname{arc}$ & * & & & & & cem & \\
\hline Azolla filiculoides & & neo & * & & $\mathrm{x}$ & & & ceo & \\
\hline Baldellia ranunculoides & & nat & 2 & $\mathrm{x}$ & & & & adi & \\
\hline Ballota nigra & & $\operatorname{arc}$ & * & & $\mathrm{x}$ & $\mathrm{x}$ & & adj & сер, сеq \\
\hline Barbarea intermedia & & neo & * & & $\mathrm{x}$ & & & cer & \\
\hline Barbarea stricta & & nat & * & & $\mathrm{x}$ & & & adk & \\
\hline Barbarea vulgaris & & nat & * & & & & & ces & $300,301,364$ \\
\hline Bellis perennis & & arc & * & & & $\mathrm{x}$ & & ceu & \\
\hline Berberis vulgaris & & nat & 3 & $x$ & & & & adl & \\
\hline Berteroa incana & & neo & * & & & & & cev & \\
\hline Berula erecta & & nat & * & & & & & cew & \\
\hline Beta vulgaris ssp. maritima & $\mathrm{x}$ & nat & $\mathrm{R}$ & & $\mathrm{x}$ & & & 70 & \\
\hline Betonica officinalis & & nat & 3 & $\mathrm{x}$ & & & & adm & \\
\hline Betula nana & & nat & 2 & $\mathrm{x}$ & & & & adn & \\
\hline Betula pendula & & nat & * & & & & & cex & \\
\hline Betula pubescens & & nat & * & $\mathrm{x}$ & & & & eat & cey, cez \\
\hline Bidens cernua & & nat & * & & $\mathrm{x}$ & & & $\mathrm{cfa}$ & \\
\hline Bidens connata & & neo & * & & $\mathrm{x}$ & & & $\mathrm{cfb}$ & \\
\hline Bidens frondosa & & neo & * & & $\mathrm{x}$ & & & $\mathrm{cfc}$ & \\
\hline Bidens radiata & & nat & * & & $\mathrm{x}$ & & & cfd & \\
\hline Bidens tripartita & & nat & * & & $\hat{x}$ & & & cfe & \\
\hline
\end{tabular}


Table A.2 - Continued from previous page

\begin{tabular}{|c|c|c|c|c|c|c|c|c|c|}
\hline $\begin{array}{l}\stackrel{\mathscr{J}}{\tilde{J}} \\
\text { के }\end{array}$ & $\underset{j}{\stackrel{0}{0}}$ & 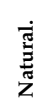 & $\begin{array}{l}\vec{Z} \\
\vec{z}\end{array}$ & $\begin{array}{l}\text { ? } \\
\mathbf{z} \\
z\end{array}$ & $\begin{array}{l}\text { à } \\
\mathbf{z} \\
\text { 嵓 }\end{array}$ & 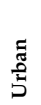 & 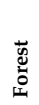 & نั & 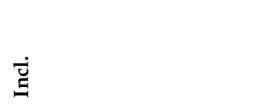 \\
\hline Biscutella laevigata ssp. guestphalica & & nat & $\mathrm{R}$ & & & & & adp & \\
\hline Bistorta officinalis & & nat & $\mathrm{V}$ & & & & & azg & \\
\hline Blechnum spicant & & nat & * & $\mathrm{x}$ & & & $\mathrm{x}$ & adq & \\
\hline Blysmus compressus & & nat & 1 & $\mathrm{x}$ & & & & adr & \\
\hline Blysmus rufus & $\mathrm{x}$ & nat & 2 & & & & & ads & \\
\hline Bolboschoenus maritimus agg. & & nat & * & & & & & 398 & adt, dpx \\
\hline Botrychium lunaria & & nat & 2 & $\mathrm{x}$ & & & & adu & \\
\hline Botrychium matricariifolium & & nat & 1 & $\mathrm{x}$ & & & & adv & \\
\hline Brachypodium pinnatum & & nat & * & & & & & $\mathrm{cfh}$ & \\
\hline Brachypodium sylvaticum & & nat & * & & & & $\mathrm{x}$ & cfi & \\
\hline Brassica nigra & & neo & * & & $\mathrm{x}$ & & & $\mathrm{cfl}$ & \\
\hline Briza media & & nat & $\mathrm{V}$ & $\mathrm{x}$ & & & & ady & \\
\hline Bromus arvensis & & arc & 3 & & & & & adz & \\
\hline Bromus benekenii & & nat & * & & & & $\mathrm{x}$ & aea & \\
\hline Bromus commutatus & & $\operatorname{arc}$ & * & $\mathrm{x}$ & & & & aeb & \\
\hline Bromus erectus & & nat & * & $\mathrm{x}$ & & & & aec & \\
\hline Bromus hordeaceus agg. & & nat & * & & & & & eau & $\mathrm{cfm}$ \\
\hline Bromus inermis & & nat & * & & & & & cfo & \\
\hline Bromus racemosus & & nat & 2 & & & & & aed & \\
\hline Bromus ramosus & & nat & * & & & & $\mathrm{x}$ & $\mathrm{cfq}$ & \\
\hline Bromus secalinus & & $\operatorname{arc}$ & * & & & & & $\mathrm{cfr}$ & \\
\hline Bromus sterilis & & arc & * & & & & & cfs & \\
\hline Bromus tectorum & & arc & * & & & & & $\mathrm{cft}$ & \\
\hline Bromus thominii & & nat & * & $\mathrm{x}$ & & & & $\mathrm{cfn}$ & \\
\hline Bryonia alba & & $\operatorname{arc}$ & 3 & & & & & aee & \\
\hline Bryonia dioica & & nat & $\mathrm{V}$ & & & & & aef & \\
\hline Bunias orientalis & & neo & * & & & & & cfu & \\
\hline Bunium bulbocastanum & & arc & 2 & & & & & aei & \\
\hline Bupleurum falcatum & & nat & 3 & $\mathrm{x}$ & & & & aej & \\
\hline Bupleurum longifolium & & nat & 3 & & & & $\mathrm{x}$ & aek & \\
\hline Bupleurum rotundifolium & & arc & 1 & & & & & ael & \\
\hline Bupleurum tenuissimum & & nat & 2 & & & & & aem & \\
\hline Butomus umbellatus & & nat & 3 & & $\mathrm{x}$ & & & aen & \\
\hline Cakile maritima & & nat & * & & $\mathrm{x}$ & & & $\mathrm{cfv}$ & \\
\hline Calamagrostis arundinacea & & nat & * & & & & $\mathrm{x}$ & aeo & \\
\hline Calamagrostis canescens & & nat & * & & & & & $\mathrm{cfw}$ & \\
\hline Calamagrostis epigejos & & nat & * & & & & & $\mathrm{cfx}$ & \\
\hline Calamagrostis phragmitoides & & nat & $\mathrm{R}$ & $\mathrm{x}$ & & & & 68 & \\
\hline Calamagrostis stricta & & nat & $\mathrm{R}$ & $\mathrm{x}$ & & & & aep & \\
\hline Calamagrostis varia & $\mathrm{x}$ & nat & $\mathrm{R}$ & $\mathrm{x}$ & & & & aeq & \\
\hline Calamagrostis villosa & & nat & * & $\mathrm{x}$ & & & & cfy & \\
\hline Calla palustris & & nat & 3 & & & & & aer & \\
\hline Callitriche palustris agg. & & nat & * & & & & & eay & aes, cfz, cga, aeu, cgb, cgc \\
\hline Calluna vulgaris & & nat & * & $\mathrm{x}$ & & & & $\operatorname{cgd}$ & \\
\hline Caltha palustris & & nat & 3 & & & & & cge & \\
\hline Calystegia sepium & & nat & * & & $\mathrm{x}$ & & & cgg & \\
\hline Calystegia soldanella & $\mathrm{x}$ & nat & 1 & & & & & aev & \\
\hline Camelina microcarpa & & arc & 2 & & & & & aex & \\
\hline Campanula bononiensis & $\mathrm{x}$ & nat & 1 & $\mathrm{x}$ & & & & aez & \\
\hline Campanula cervicaria & & nat & 1 & & & & & afa & \\
\hline Campanula glomerata & & nat & 2 & $\mathrm{x}$ & & & & $\mathrm{afb}$ & \\
\hline Campanula latifolia & & nat & 3 & & $\mathrm{x}$ & & $\mathrm{x}$ & afc & \\
\hline Campanula patula & & nat & 3 & & & & & afd & \\
\hline Campanula persicifolia & & nat & * & $\mathrm{x}$ & & & $\mathrm{x}$ & afe & \\
\hline Campanula rapunculoides & & nat & * & & & & & $\operatorname{cgh}$ & \\
\hline Campanula rapunculus & & nat & * & & & & & aff & \\
\hline Campanula rotundifolia & & nat & * & $\mathrm{x}$ & & & & cgi & \\
\hline Campanula trachelium & & nat & * & & $\mathrm{x}$ & & $\mathrm{x}$ & afg & \\
\hline Capsella bursa-pastoris & & nat & * & & & & & cgj & \\
\hline Cardamine amara & & nat & * & & & & $\mathrm{x}$ & $\mathrm{cgk}$ & \\
\hline Cardamine bulbifera & & nat & * & & & & $\mathrm{x}$ & $\mathrm{cgl}$ & \\
\hline Cardamine dentata & & nat & * & & & & & $\operatorname{cgp}$ & \\
\hline Cardamine flexuosa & & nat & * & & & & $\mathrm{x}$ & $\mathrm{cgm}$ & \\
\hline Cardamine hirsuta & & nat & * & & $\mathrm{x}$ & & & $\operatorname{cgn}$ & \\
\hline Cardamine impatiens & & nat & $*$ & & $x$ & & $\mathrm{x}$ & cgo & \\
\hline Cardamine parviflora & & nat & $\mathrm{R}$ & & & & & afh & \\
\hline Cardamine pratensis agg. & & nat & * & & & & & eaz & $\operatorname{cgq}$ \\
\hline Cardaminopsis arenosa & & neo & * & $\mathrm{x}$ & & & & $\operatorname{cgr}$ & \\
\hline Cardaminopsis halleri & & nat & * & & & & & afi & \\
\hline Cardaminopsis petraea & $\mathrm{x}$ & nat & $\mathrm{R}$ & $\mathrm{x}$ & & & & afj & \\
\hline Cardaria draba & & neo & * & & & & & $\operatorname{cgs}$ & \\
\hline Carduus acanthoides & & arc & * & & $\mathrm{x}$ & & & cgt & \\
\hline Carduus crispus & & nat & * & & $\mathrm{x}$ & & & ebb & \\
\hline
\end{tabular}


Table A.2 - Continued from previous page

\begin{tabular}{|c|c|c|c|c|c|c|c|c|c|}
\hline 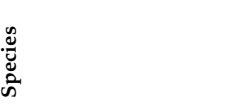 & 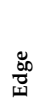 & 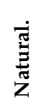 & $\begin{array}{l}\tilde{z} \\
\stackrel{a}{\approx}\end{array}$ & $\begin{array}{l}\stackrel{p}{\vec{z}} \\
\dot{z}\end{array}$ & $\begin{array}{l}a \\
\stackrel{a}{z} \\
z\end{array}$ & 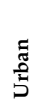 & 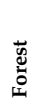 & نँ & $\begin{array}{l}\dot{\Xi} \\
\dot{\Xi}\end{array}$ \\
\hline Carduus nutans & & arc & * & & & & & afk & \\
\hline Carex acuta & & nat & * & & & & & $\mathrm{cgw}$ & \\
\hline Carex acutiformis & & nat & * & & & & & $\operatorname{cg} x$ & \\
\hline Carex appropinquata & & nat & 2 & & & & & afl & \\
\hline Carex aquatilis & & nat & 3 & & & & & $\mathrm{afm}$ & \\
\hline Carex arenaria agg. & & nat & * & & & & & ebc & cgy \\
\hline Carex bohemica & & nat & 1 & & & & & afp & \\
\hline Carex brizoides & & nat & * & $\mathrm{x}$ & & & $\mathrm{x}$ & $\operatorname{cgz}$ & \\
\hline Carex canescens & & nat & * & $\mathrm{x}$ & & & & cha & \\
\hline Carex caryophyllea & & nat & * & $\mathrm{x}$ & & & & afq & \\
\hline Carex cespitosa & & nat & 2 & & & & & afr & \\
\hline Carex demissa & & nat & $\mathrm{V}$ & $\mathrm{x}$ & & & & che & \\
\hline Carex diandra & & nat & 2 & $\mathrm{x}$ & & & & $a f v$ & \\
\hline Carex digitata & & nat & * & & & & $\mathrm{x}$ & $a f w$ & \\
\hline Carex dioica & & nat & 1 & $\mathrm{x}$ & & & & afx & \\
\hline Carex distans & & nat & $\mathrm{V}$ & & & & & afy & \\
\hline Carex disticha & & nat & * & & & & & chd & \\
\hline Carex echinata & & nat & $\mathrm{V}$ & $\mathrm{x}$ & & & & afz & \\
\hline Carex elata & & nat & 3 & & & & & chg & \\
\hline Carex elongata & & nat & 3 & & & & $\mathrm{x}$ & chh & \\
\hline Carex ericetorum & & nat & 2 & $\mathrm{x}$ & & & $x$ & aga & \\
\hline Carex extensa & & nat & * & & & & & agb & \\
\hline Carex flacca & & nat & * & & & & & agc & \\
\hline Carex flava & & nat & 3 & $\mathrm{x}$ & & & & $\operatorname{agd}$ & \\
\hline Carex hartmanii & & nat & 2 & $\mathrm{x}$ & & & & age & \\
\hline Carex hirta & & nat & * & & & & & chi & \\
\hline Carex hostiana & & nat & 1 & $\mathrm{x}$ & & & & agf & \\
\hline Carex humilis & & nat & 3 & $\mathrm{x}$ & & & & agg & \\
\hline Carex lasiocarpa & & nat & 3 & $\mathrm{x}$ & & & & agh & \\
\hline Carex lepidocarpa & & nat & 2 & $\mathrm{x}$ & & & & agi & \\
\hline Carex ligerica & & nat & * & $\mathrm{x}$ & & & & agj & \\
\hline Carex limosa & & nat & 1 & $\mathrm{x}$ & & & & agk & \\
\hline Carex montana & & nat & * & $\mathrm{x}$ & & & $\mathrm{x}$ & agl & \\
\hline Carex muricata agg. & & nat & * & & & & & ebe & chf, chk, chs \\
\hline Carex nigra & & nat & * & $\mathrm{x}$ & & & & chl & \\
\hline Carex ornithopoda & & nat & 2 & $\mathrm{x}$ & & & & agn & \\
\hline Carex otrubae & & nat & * & & & & & chb & \\
\hline Carex ovalis & & nat & * & $\mathrm{x}$ & & & & $\mathrm{chm}$ & \\
\hline Carex pallescens & & nat & * & $\mathrm{x}$ & & & & ago & \\
\hline Carex panicea & & nat & 3 & & & & & agp & \\
\hline Carex paniculata & & nat & * & & & & & chn & \\
\hline Carex pauciflora & $\mathrm{x}$ & nat & 2 & $\mathrm{x}$ & & & & agq & \\
\hline Carex pendula & & nat & * & & & & $\mathrm{x}$ & cho & \\
\hline Carex pilosa & & nat & $\mathrm{R}$ & & & & $\hat{x}$ & agr & \\
\hline Carex pilulifera & & nat & * & $\mathrm{x}$ & & & & chp & \\
\hline Carex praecox & & nat & 3 & & & & & ags & \\
\hline Carex pseudobrizoides & & nat & 2 & $\mathrm{x}$ & & & & agt & \\
\hline Carex pseudocyperus & & nat & * & & & & & agu & \\
\hline Carex pulicaris & & nat & 1 & $\mathrm{x}$ & & & & agv & \\
\hline Carex punctata & $\mathrm{x}$ & nat & 2 & $\hat{x}$ & & & & agw & \\
\hline Carex remota & & nat & * & & & & $\mathrm{x}$ & chq & \\
\hline Carex riparia & & nat & * & & & & & chr & \\
\hline Carex rostrata & & nat & * & $\mathrm{x}$ & & & & agx & \\
\hline Carex strigosa & & nat & * & & & & $\mathrm{x}$ & cht & \\
\hline Carex sylvatica & & nat & * & & & & $\mathrm{x}$ & chu & \\
\hline Carex tomentosa & & nat & 2 & & & & & aha & \\
\hline Carex trinervis & $\mathrm{x}$ & nat & 2 & $\mathrm{x}$ & & & & ahb & \\
\hline Carex umbrosa & & nat & 2 & & & & $x$ & ahc & \\
\hline Carex vesicaria & & nat & $\mathrm{V}$ & & & & & chv & \\
\hline Carex viridula & & nat & $\mathrm{V}$ & $\mathrm{x}$ & & & & agm & agy \\
\hline Carex vulpina agg. & & nat & 3 & & & & & ebg & chw \\
\hline Carex $\mathrm{x}$ beckmanniana & & nat & * & & & & & 97 & \\
\hline Carex $\mathrm{x}$ elytroides & & nat & * & & & & & $\operatorname{ch} x$ & \\
\hline Carex $\mathrm{x}$ involuta & & nat & * & & & & & 228 & \\
\hline Carex $\times$ turfosa & & nat & $\mathrm{R}$ & & & & & 98 & \\
\hline Carlina acaulis ssp. simplex & & nat & 2 & $\mathrm{x}$ & & & & ahd & \\
\hline Carlina vulgaris & & nat & * & $\mathrm{x}$ & & & & ahe & \\
\hline Carpinus betulus & & nat & * & & & & & chy & \\
\hline Carum carvi & & nat & 3 & & & & & ahf & \\
\hline Catabrosa aquatica & & nat & 2 & & $x$ & & & ahg & \\
\hline Caucalis platycarpos & & arc & 2 & & & & & ahh & \\
\hline Centaurea cyanus & & $\operatorname{arc}$ & * & & & & & ahi & \\
\hline Centaurea jacea & & nat & * & & & & & cic & \\
\hline
\end{tabular}


Table A.2 - Continued from previous page

\begin{tabular}{|c|c|c|c|c|c|c|c|c|c|}
\hline $\begin{array}{l}\stackrel{\mathscr{J}}{\tilde{J}} \\
\text { के }\end{array}$ & $\underset{j}{\stackrel{0}{0}}$ & 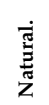 & $\begin{array}{l}\vec{Z} \\
\vec{z}\end{array}$ & $\begin{array}{l}\text { ? } \\
\mathbf{z} \\
z\end{array}$ & $\begin{array}{l}\text { à } \\
\mathbf{z} \\
⿱ 亠 䒑\end{array}$ & 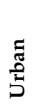 & $\begin{array}{l}\vec{w} \\
\stackrel{d}{0} \\
\dot{I}\end{array}$ & రั & 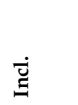 \\
\hline Centaurea montana & & nat & 2 & & & & & ahj & \\
\hline Centaurea pseudophrygia & & nat & $\mathrm{V}$ & & & & & ahk & \\
\hline Centaurea scabiosa & & nat & * & & & & & cif & \\
\hline Centaurium erythraea & & nat & * & & & & & $\mathrm{ahm}$ & \\
\hline Centaurium littorale & & nat & * & $\mathrm{x}$ & & & & ahn & cih, cii \\
\hline Centaurium pulchellum & & nat & * & & & & & aho & \\
\hline Cephalanthera damasonium & & nat & * & & & & $\mathrm{x}$ & ahq & \\
\hline Cephalanthera longifolia & & nat & 2 & & & & $\mathrm{x}$ & ahr & \\
\hline Cephalanthera rubra & & nat & 2 & & & & $\mathrm{x}$ & ahs & \\
\hline Cerastium arvense & & nat & * & & & & & cij & \\
\hline Cerastium brachypetalum & & arc & 2 & $\mathrm{x}$ & & & & aht & \\
\hline Cerastium diffusum & $\mathrm{x}$ & nat & 3 & $\mathrm{x}$ & & & & ahu & \\
\hline Cerastium dubium & & nat & 3 & & & & & ahv & \\
\hline Cerastium glomeratum & & arc & * & & & & & cik & \\
\hline Cerastium glutinosum & & nat & * & & & & & ahw & \\
\hline Cerastium holosteoides & & nat & * & & & & & cil & \\
\hline Cerastium pumilum agg. & & nat & * & $\mathrm{x}$ & & & & ebh & ahx \\
\hline Cerastium semidecandrum & & nat & * & & & & & ahy & \\
\hline Ceratocapnos claviculata & & nat & * & & & & $\mathrm{x}$ & $\operatorname{cin}$ & \\
\hline Ceratophyllum demersum & & nat & * & & $\mathrm{x}$ & & & cio & \\
\hline Ceratophyllum submersum & & nat & * & & $\mathrm{x}$ & & & ahz & \\
\hline Chaenorhinum minus & & arc & * & & & & & ciq & \\
\hline Chaerophyllum aureum & & nat & * & & $\mathrm{x}$ & & & aib & \\
\hline Chaerophyllum bulbosum & & nat & * & & $\mathrm{x}$ & & & aic & \\
\hline Chaerophyllum hirsutum & & nat & * & & $\mathrm{x}$ & & & cir & \\
\hline Chaerophyllum temulum & & nat & * & & $\mathrm{x}$ & & $\mathrm{x}$ & cis & \\
\hline Chelidonium majus & & nat & * & & $\mathrm{x}$ & $\mathrm{x}$ & & cit & \\
\hline Chenopodium album agg. & & $\operatorname{arc}$ & * & & & & & ebi & cje, cjd \\
\hline Chenopodium bonus-henricus & & nat & 3 & & $\mathrm{x}$ & $\mathrm{x}$ & & aid & \\
\hline Chenopodium ficifolium & & arc & * & & $\mathrm{x}$ & & & cix & \\
\hline Chenopodium foliosum & $\mathrm{x}$ & neo & * & & $\mathrm{x}$ & & & ciy & \\
\hline Chenopodium glaucum & & $\operatorname{arc}$ & * & & $\mathrm{x}$ & & & ciz & \\
\hline Chenopodium hybridum & & arc & 3 & & $\mathrm{x}$ & & & aie & \\
\hline Chenopodium murale & & $\operatorname{arc}$ & 3 & & $\mathrm{x}$ & $\mathrm{x}$ & & aif & \\
\hline Chenopodium polyspermum & & nat & * & & $\mathrm{x}$ & & & $\mathrm{cjb}$ & \\
\hline Chenopodium rubrum agg. & & nat & * & & & & & ebj & cjc \\
\hline Chenopodium urbicum & & arc & 1 & & $\mathrm{x}$ & $\mathrm{x}$ & & aig & \\
\hline Chenopodium vulvaria & & $\operatorname{arc}$ & 1 & & $\mathrm{x}$ & $\mathrm{x}$ & & aih & \\
\hline Chimaphila umbellata & $\mathrm{x}$ & nat & 1 & $\mathrm{x}$ & & & $\mathrm{x}$ & aii & \\
\hline Chondrilla juncea & & nat & 3 & & & & & aij & \\
\hline Chrysanthemum segetum & & arc & * & & & & & aik & \\
\hline Chrysosplenium alternifolium & & nat & * & & & & $\mathrm{x}$ & ail & \\
\hline Chrysosplenium oppositifolium & & nat & * & & & & $x$ & aim & \\
\hline Cicendia filiformis & & nat & 2 & $\mathrm{x}$ & & & & ain & \\
\hline Cicerbita alpina & & nat & 3 & & $\mathrm{x}$ & & & aio & \\
\hline Cichorium intybus & & arc & * & & & & & aip & \\
\hline Cicuta virosa & & nat & 3 & & & & & aiq & \\
\hline Circaea alpina & & nat & * & & & & $\mathrm{x}$ & air & \\
\hline Circaea lutetiana & & nat & * & & $\mathrm{x}$ & & $\mathrm{x}$ & cjf & \\
\hline Circaea $\mathrm{x}$ intermedia & & nat & * & & & & $\mathrm{x}$ & ais & \\
\hline Cirsium acaule & & nat & * & $\mathrm{x}$ & & & & ait & \\
\hline Cirsium arvense & & nat & * & & $\mathrm{x}$ & & & cjg & \\
\hline Cirsium dissectum & & nat & 2 & $\mathrm{x}$ & & & & aiu & \\
\hline Cirsium eriophorum & & nat & 3 & & & & & aiv & \\
\hline Cirsium oleraceum & & nat & * & & & & & cjh & \\
\hline Cirsium palustre & & nat & * & $\mathrm{x}$ & & & & cji & \\
\hline Cirsium vulgare & & nat & * & & $\mathrm{x}$ & & & $\mathrm{cjj}$ & \\
\hline Cirsium $\mathrm{x}$ rigens & & nat & * & & & & & 9 & \\
\hline Cladium mariscus & & nat & 2 & $\mathrm{x}$ & & & & aix & \\
\hline Claytonia perfoliata & & neo & * & & $\mathrm{x}$ & $\mathrm{x}$ & & $c j k$ & \\
\hline Clematis vitalba & & nat & * & & $\mathrm{x}$ & & & cjl & \\
\hline Clinopodium vulgare & & nat & * & $\mathrm{x}$ & & & & aiz & \\
\hline Cnidium dubium & & $\operatorname{arc}$ & 2 & & & & & aja & \\
\hline Cochlearia anglica & & nat & $\mathrm{V}$ & & $\mathrm{x}$ & & & cjm & \\
\hline Cochlearia danica & & nat & * & & & & & cjn & \\
\hline Cochlearia officinalis & & nat & 1 & & & & & ajb & \\
\hline Colchicum autumnale & & nat & 3 & & & & & ajd & \\
\hline Conium maculatum & & $\operatorname{arc}$ & * & & $\mathrm{x}$ & $\mathrm{x}$ & & aje & \\
\hline Conopodium majus & & neo & * & & & & & ajf & \\
\hline Consolida regalis & & $\operatorname{arc}$ & 3 & & & & & ajh & \\
\hline Convallaria majalis & & nat & * & & & & $\mathrm{x}$ & aji & \\
\hline Convolvulus arvensis & & nat & * & & & & & cjr & \\
\hline Conyza canadensis & & neo & * & & & & & cjs & \\
\hline
\end{tabular}


Table A.2 - Continued from previous page

\begin{tabular}{|c|c|c|c|c|c|c|c|c|c|}
\hline 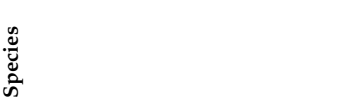 & 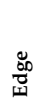 & 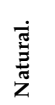 & $\begin{array}{l}\stackrel{\bullet}{Z} \\
\stackrel{z}{\approx}\end{array}$ & $\begin{array}{l}\stackrel{p}{\vec{z}} \\
\dot{z}\end{array}$ & 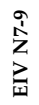 & 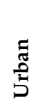 & 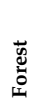 & نँ & $\begin{array}{l}\dot{\Xi} \\
\dot{\Xi}\end{array}$ \\
\hline Corispermum leptopterum & & neo & * & & & & & ajk & \\
\hline Cornus mas & & nat & 3 & & & & $\mathrm{x}$ & ajl & \\
\hline Cornus sanguinea & & nat & * & & & & & cju & \\
\hline Cornus suecica & & nat & 1 & $\mathrm{x}$ & & & & ajm & \\
\hline Coronilla coronata & & nat & 2 & $\mathrm{x}$ & & & $\mathrm{x}$ & ajn & \\
\hline Coronopus squamatus & & arc & 3 & & & & & ajp & \\
\hline Corrigiola litoralis & & nat & 3 & & & & & ajq & \\
\hline Corydalis cava & & nat & * & & $\mathrm{x}$ & & $\mathrm{x}$ & ajr & \\
\hline Corydalis intermedia & & nat & * & & $\mathrm{x}$ & & $\mathrm{x}$ & ajs & \\
\hline Corydalis solida & & nat & * & & $\mathrm{x}$ & & $\mathrm{x}$ & ajt & \\
\hline Corylus avellana & & nat & * & & & & & cjw & \\
\hline Corynephorus canescens & & nat & * & $\mathrm{x}$ & & & & cjx & \\
\hline Cotoneaster integerrimus & & nat & $\mathrm{R}$ & $\mathrm{x}$ & & & & aju & \\
\hline Cotula coronopifolia & & neo & 3 & & $\mathrm{x}$ & & & ajv & \\
\hline Crambe maritima & & nat & $\mathrm{R}$ & & $\mathrm{x}$ & & & ajw & \\
\hline Crataegus laevigata agg. & & nat & * & & & & & ebl & ebm, cka, ckb \\
\hline Crataegus monogyna & & nat & * & & & & & ebo & cke, ckf \\
\hline Crataegus rhipidophylla & & nat & * & & & & $\mathrm{x}$ & cjz & ebk \\
\hline Crataegus x macrocarpa & & nat & * & & & & & $\mathrm{ckd}$ & \\
\hline Crataegus x media & & nat & * & & & & & 21 & \\
\hline Crepis biennis & & arc & * & & & & & ajy & \\
\hline Crepis capillaris & & arc & * & & & & & $\mathrm{ckg}$ & \\
\hline Crepis foetida & & arc & 2 & $\mathrm{x}$ & & & & ajz & \\
\hline Crepis mollis & & nat & 2 & & & & & aka & \\
\hline Crepis paludosa & & nat & * & & & & & $\mathrm{ckh}$ & \\
\hline Crepis praemorsa & & nat & 1 & $\mathrm{x}$ & & & & $\mathrm{akb}$ & \\
\hline Crepis tectorum & & nat & $\mathrm{V}$ & & & $\mathrm{x}$ & & akd & \\
\hline Crepis vesicaria ssp. taraxacifolia & & nat & * & & & & & akc & \\
\hline Cruciata laevipes & & nat & * & & $\mathrm{x}$ & & & ckj & \\
\hline Cucubalus baccifer & & nat & 2 & & $\mathrm{x}$ & & $\mathrm{x}$ & akf & \\
\hline Cuscuta campestris & & neo & * & & & & & 19 & \\
\hline Cuscuta epithymum & & nat & 2 & $\mathrm{x}$ & & & & akh & \\
\hline Cuscuta europaea & & nat & * & & $\mathrm{x}$ & & & ckk & \\
\hline Cuscuta lupuliformis & & neo & * & & $\mathrm{x}$ & & & aki & \\
\hline Cymbalaria muralis & & neo & * & & & $\mathrm{x}$ & & akj & \\
\hline Cynoglossum germanicum & & nat & 3 & & $\mathrm{x}$ & & $\mathrm{x}$ & akk & \\
\hline Cynoglossum officinale & & nat & 3 & & $\mathrm{x}$ & & & akl & \\
\hline Cynosurus cristatus & & nat & * & & & & & cko & \\
\hline Cyperus fuscus & & nat & 3 & & & & & akn & \\
\hline Cypripedium calceolus & & nat & 2 & & & & $\mathrm{x}$ & ako & \\
\hline Cystopteris fragilis & & nat & * & & & & & akp & \\
\hline Cytisus scoparius & & nat & * & & & & & $\mathrm{ckq}$ & \\
\hline Dactylis glomerata & & nat & * & & & & & $\mathrm{ckr}$ & \\
\hline Dactylis polygama & & nat & * & & & & $\mathrm{x}$ & cks & \\
\hline Dactylorhiza incarnata ssp. incarnata & & nat & 2 & $\mathrm{x}$ & & & & akq & \\
\hline Dactylorhiza maculata agg. & & nat & 3 & $\mathrm{x}$ & & & & akr & ebq \\
\hline Dactylorhiza majalis & & nat & 2 & $\mathrm{x}$ & & & & akt & \\
\hline Dactylorhiza praetermissa & & nat & 3 & $\mathrm{x}$ & & & & aku & \\
\hline Dactylorhiza sphagnicola & & nat & 2 & $\mathrm{x}$ & & & & akv & \\
\hline Dactylorhiza $\times$ dinglensis & & nat & $\mathrm{n} / \mathrm{a}$ & & & & & dpm & \\
\hline Danthonia decumbens & & nat & $\mathrm{V}$ & $\mathrm{x}$ & & & & ckt & \\
\hline Daphne mezereum & & nat & * & & & & $\mathrm{x}$ & akw & \\
\hline Datura stramonium & & neo & * & & $\mathrm{x}$ & & & akx & \\
\hline Daucus carota & & nat & * & & & & & $\mathrm{cku}$ & \\
\hline Deschampsia cespitosa & & nat & * & $\mathrm{x}$ & & & & $\mathrm{ckv}$ & \\
\hline Deschampsia flexuosa & & nat & * & $\mathrm{x}$ & & & & ckw & \\
\hline Deschampsia setacea & & nat & 1 & $\mathrm{x}$ & & & & aky & \\
\hline Deschampsia wibeliana & & nat & 3 & & & & & akz & \\
\hline Descurainia sophia & & arc & * & & & & & ckx & \\
\hline Dianthus armeria & & nat & 3 & $\mathrm{x}$ & & & & ala & \\
\hline Dianthus carthusianorum & & nat & 3 & $\mathrm{x}$ & & & & alb & \\
\hline Dianthus deltoides & & nat & 3 & $\mathrm{x}$ & & & & alc & \\
\hline Dianthus gratianopolitanus & & nat & $\mathrm{R}$ & $\mathrm{x}$ & & & & ald & \\
\hline Dianthus superbus & & nat & 1 & $\mathrm{x}$ & & & & ale & \\
\hline Digitalis grandiflora & & nat & 2 & & & & $\mathrm{x}$ & alg & \\
\hline Digitalis purpurea & & nat & * & & & & $x$ & cky & \\
\hline Digitaria ischaemum & & arc & * & $\mathrm{x}$ & & & & $\mathrm{ckz}$ & \\
\hline Digitaria sanguinalis & & arc & * & & & & & alh & \\
\hline Diphasiastrum alpinum & & nat & 2 & $\mathrm{x}$ & & & & ali & \\
\hline Diphasiastrum complanatum & & nat & 2 & $\mathrm{x}$ & & & $\mathrm{x}$ & alj & \\
\hline Diphasiastrum issleri & & nat & 2 & $\mathrm{x}$ & & & & alk & \\
\hline Diphasiastrum tristachyum & & nat & 2 & $\mathrm{x}$ & & & & all & \\
\hline Diphasiastrum zeilleri & & nat & 2 & $\mathrm{x}$ & & & & alm & \\
\hline
\end{tabular}


Table A.2 - Continued from previous page

\begin{tabular}{|c|c|c|c|c|c|c|c|c|c|}
\hline 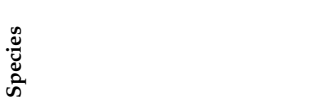 & 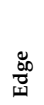 & 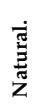 & $\begin{array}{l}\stackrel{\text { Z }}{a} \\
\underset{\approx}{2}\end{array}$ & $\begin{array}{l}\stackrel{?}{1} \\
\dot{z} \\
\text { 㭊 }\end{array}$ & $\begin{array}{l}\text { I } \\
\text { 乙 } \\
\text { 空 }\end{array}$ & $\begin{array}{l}\text { శ్ } \\
\text { 莡 } \\
\end{array}$ & 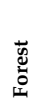 & نँ & 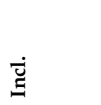 \\
\hline Diplotaxis muralis & & neo & * & & & & & cla & \\
\hline Diplotaxis tenuifolia & & neo & * & & & $\mathrm{x}$ & & $\mathrm{clb}$ & \\
\hline Dipsacus fullonum & & $\operatorname{arc}$ & * & & $\mathrm{x}$ & & & aln & \\
\hline Dipsacus pilosus & & nat & * & & $\mathrm{x}$ & & $\mathrm{x}$ & cld & \\
\hline Draba muralis & & neo & * & & & $\mathrm{x}$ & & clf & \\
\hline Drosera intermedia & & nat & 3 & $\mathrm{x}$ & & & & alp & \\
\hline Drosera longifolia & & nat & 1 & $\mathrm{x}$ & & & & alo & \\
\hline Drosera rotundifolia & & nat & 3 & $\mathrm{x}$ & & & & alr & \\
\hline Drosera $\times$ obovata & & nat & 1 & $\mathrm{x}$ & & & & alq & \\
\hline Dryopteris affinis & & nat & * & & & & $\mathrm{x}$ & als & \\
\hline Dryopteris carthusiana & & nat & * & $\mathrm{x}$ & & & $\mathrm{x}$ & cli & \\
\hline Dryopteris cristata & & nat & 3 & & & & & alt & \\
\hline Dryopteris dilatata & & nat & * & & $\mathrm{x}$ & & $\mathrm{x}$ & clj & \\
\hline Dryopteris expansa & & nat & $\mathrm{R}$ & $\mathrm{x}$ & & & $\mathrm{x}$ & clk & \\
\hline Dryopteris filix-mas & & nat & * & & & & $\mathrm{x}$ & cll & \\
\hline Echinochloa crus-galli & & arc & * & & $\mathrm{x}$ & & & $\mathrm{clm}$ & \\
\hline Echinochloa muricata & & neo & * & & & & & 47 & \\
\hline Echinops sphaerocephalus & & neo & * & & $\mathrm{x}$ & & & clp & \\
\hline Echium vulgare & & arc & * & & & & & alv & \\
\hline Elatine hexandra & & nat & 2 & $\mathrm{x}$ & & & & alx & \\
\hline Elatine hydropiper & & nat & 2 & $\mathrm{x}$ & & & & aly & \\
\hline Elatine triandra & & nat & 2 & & & & & alz & \\
\hline Eleocharis acicularis & & nat & 3 & $\mathrm{x}$ & & & & ama & \\
\hline Eleocharis austriaca & & nat & $\mathrm{R}$ & & & & & ebt & \\
\hline Eleocharis mamillata & & nat & 3 & $\mathrm{x}$ & & & & $\mathrm{amb}$ & \\
\hline Eleocharis multicaulis & & nat & 2 & $\hat{x}$ & & & & amc & \\
\hline Eleocharis ovata & & nat & 1 & & & & & amd & \\
\hline Eleocharis palustris & & nat & * & & & & & clr & ebu, ebv \\
\hline Eleocharis quinqueflora & & nat & 3 & $\mathrm{x}$ & & & & $\mathrm{amf}$ & \\
\hline Eleocharis uniglumis & & nat & * & & & & & amg & \\
\hline Elodea canadensis & & neo & * & & $\mathrm{x}$ & & & $\mathrm{cls}$ & \\
\hline Elodea nuttallii & & neo & * & & $\mathrm{x}$ & & & clt & \\
\hline Elymus athericus & & nat & * & & & & & $\mathrm{clv}$ & \\
\hline Elymus caninus & & nat & * & & $\mathrm{x}$ & & $\mathrm{x}$ & aаj & \\
\hline Elymus farctus ssp. boreoatlanticus & & nat & * & & $\mathrm{x}$ & & & clu & \\
\hline Elymus repens & & nat & * & & $\mathrm{x}$ & & & $\mathrm{clw}$ & \\
\hline Elymus $\mathrm{x}$ obtusiusculus & $\mathrm{x}$ & nat & * & & & & & 71 & \\
\hline Elymus $\mathrm{x}$ oliveri & & nat & * & & & & & $\mathrm{ctk}$ & \\
\hline Empetrum nigrum & & nat & * & $\mathrm{x}$ & & & & $\mathrm{clx}$ & \\
\hline Epilobium angustifolium & & nat & * & & $\mathrm{x}$ & & $\mathrm{x}$ & cly & \\
\hline Epilobium ciliatum & & neo & * & & $\hat{x}$ & & & $\mathrm{clz}$ & \\
\hline Epilobium collinum & & nat & 3 & $\mathrm{x}$ & & & & cma & \\
\hline Epilobium hirsutum & & nat & * & & $\mathrm{x}$ & & & $\mathrm{cmb}$ & \\
\hline Epilobium lanceolatum & & nat & $\mathrm{R}$ & $\mathrm{x}$ & & & & $\mathrm{amh}$ & \\
\hline Epilobium montanum & & nat & * & & & & & $\mathrm{cmc}$ & \\
\hline Epilobium obscurum & & nat & * & & & & & cmd & \\
\hline Epilobium palustre & & nat & * & $\mathrm{x}$ & & & & cme & \\
\hline Epilobium parviflorum & & nat & * & & & & & $\mathrm{cmf}$ & \\
\hline Epilobium roseum & & nat & $\mathrm{V}$ & & $\mathrm{x}$ & & & $\mathrm{cmg}$ & \\
\hline Epilobium tetragonum & & nat & * & & & & & ebw & $\mathrm{cmh}$ \\
\hline Epipactis atrorubens & & nat & 3 & $\mathrm{x}$ & & & & ami & \\
\hline Epipactis helleborine & & nat & * & & & & $\mathrm{x}$ & 370 & cmj, 243 \\
\hline Epipactis leptochila & & nat & 3 & & & & $\hat{x}$ & amj & $\mathrm{cmk}, \mathrm{cml}$ \\
\hline Epipactis microphylla & & nat & 3 & & & & $x$ & amk & \\
\hline Epipactis muelleri & & nat & 3 & $\mathrm{x}$ & & & $\mathrm{x}$ & $\mathrm{aml}$ & \\
\hline Epipactis palustris & & nat & 2 & $\hat{x}$ & & & $\hat{x}$ & $\mathrm{amm}$ & \\
\hline Epipactis purpurata & & nat & 3 & & & & & amn & \\
\hline Epipogium aphyllum & & nat & 1 & & & & $x$ & amo & \\
\hline Equisetum arvense & & nat & * & $\mathrm{x}$ & & & & $\mathrm{cmm}$ & \\
\hline Equisetum fluviatile & & nat & * & & & & & $\mathrm{cmn}$ & \\
\hline Equisetum hyemale & & nat & 3 & & & & $\mathrm{x}$ & $\mathrm{amp}$ & \\
\hline Equisetum palustre & & nat & * & $\mathrm{x}$ & & & & $\mathrm{cmo}$ & \\
\hline Equisetum pratense & & nat & 2 & $\mathrm{x}$ & & & $\mathrm{x}$ & amg & \\
\hline Equisetum sylvaticum & & nat & * & & & & $x$ & $\mathrm{cmp}$ & \\
\hline Equisetum telmateia & & nat & * & & & & $\hat{x}$ & amr & \\
\hline Equisetum variegatum & & nat & 1 & $\mathrm{x}$ & & & & ams & \\
\hline Equisetum $\mathrm{x}$ litorale & & nat & * & & & & & $\mathrm{cmq}$ & \\
\hline Eragrostis albensis & & nat & * & & & & & 46 & \\
\hline Eragrostis minor & & neo & * & & & $\mathrm{x}$ & & $\mathrm{cms}$ & \\
\hline Erica tetralix & & nat & $\mathrm{V}$ & $\mathrm{x}$ & & & & amt & \\
\hline Erigeron acris & & nat & * & $\hat{x}$ & & & & eby & \\
\hline Erigeron annuus & & neo & * & & $\mathrm{x}$ & & & $\mathrm{cmx}$ & 401 \\
\hline Eriophorum angustifolium & & nat & $\mathrm{V}$ & $x$ & & & & $\mathrm{amu}$ & \\
\hline
\end{tabular}


Table A.2 - Continued from previous page

\begin{tabular}{|c|c|c|c|c|c|c|c|c|c|}
\hline 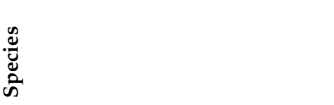 & 总 & 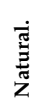 & $\begin{array}{l}\stackrel{\infty}{Z} \\
\stackrel{z}{z}\end{array}$ & $\begin{array}{l}\stackrel{p}{1} \\
\vec{Z} \\
\stackrel{z}{z}\end{array}$ & 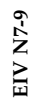 & 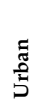 & $\begin{array}{l}\overrightarrow{0} \\
\stackrel{0}{0} \\
\dot{I}\end{array}$ & نँ & 它 \\
\hline Eriophorum gracile & & nat & 1 & $\mathrm{x}$ & & & & $a m v$ & \\
\hline Eriophorum latifolium & & nat & 1 & $\mathrm{x}$ & & & & amw & \\
\hline Eriophorum vaginatum & & nat & $\mathrm{V}$ & $\mathrm{x}$ & & & & $a m x$ & \\
\hline Erodium ballii & $\mathrm{x}$ & nat & * & $\mathrm{x}$ & & & & cmy & \\
\hline Erodium cicutarium & & nat & * & & & & & $\mathrm{cmz}$ & \\
\hline Erophila verna & & nat & * & $\mathrm{x}$ & & & & $\mathrm{cnb}$ & \\
\hline Erucastrum gallicum & & neo & * & & & & & cnd & \\
\hline Eryngium campestre & & nat & 3 & $\mathrm{x}$ & & & & amy & \\
\hline Eryngium maritimum & $\mathrm{x}$ & nat & 3 & & & & & $\mathrm{amz}$ & \\
\hline Erysimum cheiranthoides & & nat & * & & $\mathrm{x}$ & & & cne & \\
\hline Erysimum hieraciifolium & & nat & 3 & & $\mathrm{x}$ & & & ana & \\
\hline Euоnути еuropaea & & nat & * & & & & & $\mathrm{cnh}$ & \\
\hline Eupatorium cannabinum & & nat & * & & $\mathrm{x}$ & & & cni & \\
\hline Euphorbia amygdaloides & & nat & * & & & & $\mathrm{x}$ & cnj & \\
\hline Euphorbia cyparissias & & nat & * & $\mathrm{x}$ & & & & cnk & \\
\hline Euphorbia esula & & nat & * & & & & & $\mathrm{cnl}$ & \\
\hline Euphorbia exigua & & $\operatorname{arc}$ & $\mathrm{V}$ & & & & & anb & \\
\hline Euphorbia helioscopia & & arc & * & & $\mathrm{x}$ & & & $\mathrm{cnm}$ & \\
\hline Euphorbia palustris & & nat & 2 & & & & & anc & \\
\hline Euphorbia peplus & & $\operatorname{arc}$ & * & & $\mathrm{x}$ & & & cno & \\
\hline Euphorbia platyphyllos & & nat & 2 & & & & & and & \\
\hline Euphorbia $\times$ pseudovirgata & & neo & * & & & & & $\mathrm{cnp}$ & \\
\hline Euphrasia frigida & $\mathrm{x}$ & nat & $\mathrm{R}$ & $\mathrm{x}$ & & & & 248 & \\
\hline Euphrasia nemorosa agg. & & nat & 3 & & & & & anf & cnq, cnr \\
\hline Euphrasia officinalis ssp. rostkoviana & & nat & 3 & & & & & ang & \\
\hline Euphrasia stricta & & nat & * & $\mathrm{x}$ & & & & cns & \\
\hline Fagus sylvatica & & nat & * & & & & & cnu & \\
\hline Falcaria vulgaris & & nat & * & & & & & anh & \\
\hline Fallopia convolvulus & & arc & * & & & & & dah & \\
\hline Fallopia dumetorum & & nat & * & & $\mathrm{x}$ & & & daj & \\
\hline Fallopia japonica & & neo & * & & $\mathrm{x}$ & & & dai & \\
\hline Fallopia sachalinensis & & neo & * & & $\mathrm{x}$ & & & daq & \\
\hline Fallopia $\times$ bohemica & & neo & * & & & & & 64 & \\
\hline Festuca altissima & & nat & * & & & & $\mathrm{x}$ & $\mathrm{cnv}$ & \\
\hline Festuca arundinacea & & nat & * & & & & & $\mathrm{cnw}$ & \\
\hline Festuca gigantea & & nat & * & & & & $\mathrm{x}$ & cny & \\
\hline Festuca heterophylla & & nat & 3 & & & & $x$ & ani & \\
\hline Festuca ovina agg. & & nat & * & $\mathrm{x}$ & & & & ecc & coh, cnx, cnz, coa, anj, dsx, cog, 240, ank \\
\hline Festuca pratensis & & nat & * & & & & & $\mathrm{cob}$ & \\
\hline Festuca rubra agg. & & nat & * & & & & & 397 & 413, ecd, 225, coe, 54, cod \\
\hline Filago arvensis & & nat & * & $\mathrm{x}$ & & & & anl & \\
\hline Filago minima & & nat & * & $\mathrm{x}$ & & & & ann & \\
\hline Filago vulgaris & & nat & 2 & $\mathrm{x}$ & & & & ano & \\
\hline Filipendula ulmaria & & nat & * & & & & & ece & coi, coj \\
\hline Filipendula vulgaris & & nat & 2 & $\mathrm{x}$ & & & & anp & \\
\hline Fragaria moschata & & nat & 3 & & & & $\mathrm{x}$ & anq & \\
\hline Fragaria vesca & & nat & * & & & & & cok & \\
\hline Fragaria viridis & & nat & $\mathrm{V}$ & $\mathrm{x}$ & & & & anr & \\
\hline Frangula alnus & & nat & * & & & & & $\mathrm{col}$ & \\
\hline Fraxinus excelsior & & nat & * & & $\mathrm{x}$ & & & com & \\
\hline Fritillaria meleagris & & $\operatorname{arc}$ & 3 & & & & & ans & \\
\hline Fumaria officinalis & & arc & * & & $\mathrm{x}$ & & & ecg & $\operatorname{coq}$ \\
\hline Fumaria vaillantii & & arc & 3 & & & & & ant & \\
\hline Gagea bohemica ssp. saxatilis & $\mathrm{x}$ & nat & $\mathrm{R}$ & $\mathrm{x}$ & & & & anx & \\
\hline Gagea lutea & & nat & * & & $\mathrm{x}$ & & $\mathrm{x}$ & anu & \\
\hline Gagea minima & & nat & 2 & & $\mathrm{x}$ & & $\mathrm{x}$ & anv & \\
\hline Gagea pratensis & & nat & $\mathrm{V}$ & & & & & anw & \\
\hline Gagea spathacea & & nat & $\mathrm{V}$ & & $\mathrm{x}$ & & $\mathrm{x}$ & $\cos$ & \\
\hline Gagea villosa & & arc & 3 & & & & & any & \\
\hline Galanthus nivalis & & neo & * & & $\mathrm{x}$ & & $\mathrm{x}$ & $\cot$ & \\
\hline Galeopsis angustifolia & & arc & 3 & & & & & $\operatorname{cov}$ & \\
\hline Galeopsis bifida & & nat & * & & & & & cow & \\
\hline Galeopsis ladanum & & nat & 1 & $\mathrm{x}$ & & & & anz & \\
\hline Galeopsis segetum & & nat & 2 & $\mathrm{x}$ & & & & aoa & \\
\hline Galeopsis speciosa & & nat & V & & $x$ & & & aob & \\
\hline Galeopsis tetrahit & & nat & * & & & & & $\operatorname{cox}$ & \\
\hline Galinsoga ciliata & & neo & * & & $\mathrm{x}$ & & & coy & \\
\hline Galinsoga parviflora & & neo & * & & $\mathrm{x}$ & & & $\mathrm{COZ}$ & \\
\hline Galium album & & nat & * & & & & & сра & \\
\hline Galium aparine & & nat & * & & $\mathrm{x}$ & & & $\mathrm{cpb}$ & \\
\hline Galium boreale & & nat & 3 & $\mathrm{x}$ & & & & aoc & \\
\hline Galium glaucum & & nat & 1 & $x$ & & & & aod & \\
\hline Galium odoratum & & nat & * & & & & $\mathrm{x}$ & aoe & \\
\hline
\end{tabular}


Table A.2 - Continued from previous page

\begin{tabular}{|c|c|c|c|c|c|c|c|c|c|}
\hline $\begin{array}{l}\mathscr{ٌ} \\
\text { ळूँ } \\
\text { के }\end{array}$ & 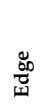 & $\frac{\tilde{\Xi}}{\stackrel{\Xi}{\tilde{z}}}$ & $\begin{array}{l}\stackrel{\text { Z }}{a} \\
\underset{\approx}{2}\end{array}$ & $\begin{array}{l}\frac{p}{1} \\
z \\
z\end{array}$ & $\begin{array}{l}\text { à } \\
\mathbf{z} \\
\text { 嵓 }\end{array}$ & 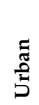 & 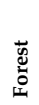 & 8: & 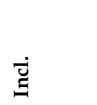 \\
\hline Galium palustre & & nat & * & & & & & $\mathrm{cpc}$ & ctx \\
\hline Galium pumilum & & nat & 3 & $\mathrm{x}$ & & & & cpe & \\
\hline Galium rotundifolium & & neo & $\mathrm{R}$ & & & & $\mathrm{x}$ & aof & \\
\hline Galium saxatile & & nat & * & $\mathrm{x}$ & & & & $\mathrm{cpf}$ & \\
\hline Galium spurium ssp. vaillantii & & arc & 2 & & & & & aog & \\
\hline Galium sylvaticum & & nat & * & & & & $\mathrm{x}$ & aoh & \\
\hline Galium uliginosum & & nat & * & $\mathrm{x}$ & & & & cpg & \\
\hline Galium verum & & nat & * & $\mathrm{x}$ & & & & $\mathrm{cph}$ & aoj \\
\hline Galium wirtgenii & & nat & 2 & & & & & aok & \\
\hline Galium $\times$ pomeranicum & & nat & $*$ & & & & & 23 & \\
\hline Genista anglica & & nat & 3 & $\mathrm{x}$ & & & & aol & \\
\hline Genista germanica & & nat & 2 & $\mathrm{x}$ & & & & aom & \\
\hline Genista pilosa & & nat & 3 & $\mathrm{x}$ & & & & aon & \\
\hline Genista tinctoria & & nat & $\mathrm{V}$ & $\mathrm{x}$ & & & & aoo & \\
\hline Gentiana cruciata & & nat & 2 & $\mathrm{x}$ & & & & aop & \\
\hline Gentiana pneumonanthe & & nat & 2 & $\mathrm{x}$ & & & & aoq & \\
\hline Gentianella ciliata & & nat & 3 & $\mathrm{x}$ & & & & aou & \\
\hline Gentianella germanica & & nat & 3 & $\mathrm{x}$ & & & & aov & \\
\hline Geranium columbinum & & arc & * & & $\mathrm{x}$ & & & $\mathrm{cpk}$ & \\
\hline Geranium dissectum & & arc & * & & & & & $\mathrm{cpl}$ & \\
\hline Geranium lucidum & & nat & $\mathrm{R}$ & & $\mathrm{x}$ & & & aox & \\
\hline Geranium molle & & arc & * & & & & & $\mathrm{cpm}$ & \\
\hline Geranium palustre & & nat & * & & $\mathrm{x}$ & & & aoy & \\
\hline Geranium pratense & & nat & $\mathrm{V}$ & & $\mathrm{x}$ & & & aoz & \\
\hline Geranium purpureum & & neo & * & & & & & 96 & \\
\hline Geranium pusillum & & arc & * & & $\mathrm{x}$ & & & сро & \\
\hline Geranium pyrenaicum & & neo & * & & $\mathrm{x}$ & $\mathrm{x}$ & & cpp & \\
\hline Geranium robertianum & & nat & * & & $\mathrm{x}$ & & & cpq & \\
\hline Geranium sanguineum & & nat & 2 & $\mathrm{x}$ & & & $\mathrm{x}$ & apa & \\
\hline Geranium sylvaticum & & nat & * & & $\mathrm{x}$ & & & apb & \\
\hline Geum rivale & & nat & 3 & & & & & apc & \\
\hline Geum urbanum & & nat & * & & $\mathrm{x}$ & & $\mathrm{x}$ & $\operatorname{cps}$ & \\
\hline Glaucium flavum & $\mathrm{x}$ & nat & $\mathrm{R}$ & & $\mathrm{x}$ & & & 146 & \\
\hline Glaux maritima & & nat & * & & & & & apd & \\
\hline Glechoma hederacea & & nat & * & & $\mathrm{x}$ & & & cpt & \\
\hline Glyceria declinata & & nat & * & & & & & cpu & \\
\hline Glyceria fluitans + & & nat & * & & $\mathrm{x}$ & & & $\mathrm{cpv}$ & \\
\hline Glyceria maxima & & nat & * & & $\mathrm{x}$ & & & $\mathrm{cpw}$ & \\
\hline Glyceria notata & & nat & * & & $\mathrm{x}$ & & & cpx & \\
\hline Gnaphalium sylvaticum & & nat & * & & & & & cpy & \\
\hline Gnaphalium uliginosum & & nat & * & & & & & cpz & \\
\hline Goodyera repens & & nat & 2 & $\mathrm{x}$ & & & $\mathrm{x}$ & apg & \\
\hline Gratiola officinalis & & nat & 2 & & & & & aph & \\
\hline Groenlandia densa & & nat & 2 & & & & & api & \\
\hline Gymnadenia conopsea & & nat & 3 & $\mathrm{x}$ & & & & apj & \\
\hline Gymnocarpium dryopteris & & nat & * & & & & $\mathrm{x}$ & apk & \\
\hline Gymnocarpium robertianum & & nat & 3 & $\mathrm{x}$ & & & & apl & \\
\hline Gypsophila muralis & & nat & 2 & $\mathrm{x}$ & & & & apm & \\
\hline Gypsophila perfoliata & & neo & * & & & & & 43 & \\
\hline Gypsophila repens & $\mathrm{x}$ & nat & $\mathrm{R}$ & $x$ & & & & apn & \\
\hline Gypsophila scorzonerifolia & & neo & * & & & & & 44 & \\
\hline Hammarbya paludosa & & nat & 1 & $\mathrm{x}$ & & & & app & \\
\hline Hedera helix & & nat & * & & & & & cqb & \\
\hline Heliantheтит питтиlarium & & nat & 3 & & & & & apq & apr, aps \\
\hline Helianthus tuberosus & & neo & * & & $\mathrm{x}$ & & & cqe & \\
\hline Helichrysum arenarium & & nat & 3 & $\mathrm{x}$ & & & & apt & \\
\hline Helictotrichon pratense & & nat & 3 & $\mathrm{x}$ & & & & adg & \\
\hline Helictotrichon pubescens & & nat & * & & & & & adh & \\
\hline Helleborus foetidus & & nat & * & $\mathrm{x}$ & & & $\mathrm{x}$ & cqf & \\
\hline Helleborus viridis & & nat & 3 & & & & $\hat{x}$ & apu & \\
\hline Hepatica nobilis & & nat & * & & & & $\mathrm{x}$ & apv & \\
\hline Heracleum mantegazzianum & & neo & * & & $\mathrm{x}$ & & & cqg & \\
\hline Heracleum sphondylium & & nat & * & & $\hat{x}$ & & & cqh & \\
\hline Herminium monorchis & & nat & 1 & $\mathrm{x}$ & & & & apw & \\
\hline Herniaria glabra & & arc & * & $\hat{x}$ & & $\mathrm{x}$ & & apx & \\
\hline Hesperis matronalis & & neo & * & & $\mathrm{x}$ & & $\mathrm{x}$ & cqj & \\
\hline Hieracium aridum & & nat & * & & & & & dsi & \\
\hline Hieracium arvicola & & nat & G & $\mathrm{x}$ & & & & $\mathrm{cql}$ & \\
\hline Hieracium aurantiacum & & neo & * & $\mathrm{x}$ & & & & apy & \\
\hline Hieracium bauhini & & nat & * & $x$ & & & & apz & \\
\hline Hieracium bifidum & & nat & 2 & $\hat{x}$ & & & & aqa & \\
\hline Hieracium bifurcum & & nat & $\mathrm{G}$ & & & & & 236 & \\
\hline Hieracium brachiatum & & nat & $*$ & & & & & cqm & \\
\hline
\end{tabular}


Table A.2 - Continued from previous page

\begin{tabular}{|c|c|c|c|c|c|c|c|c|c|}
\hline 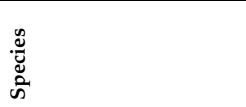 & 总 & 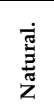 & $\begin{array}{l}\stackrel{2}{Z} \\
\vec{\approx}\end{array}$ & $\begin{array}{l}p \\
z \\
z \\
\geq\end{array}$ & $\begin{array}{l}\stackrel{i}{i} \\
\sum_{i}\end{array}$ & 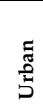 & 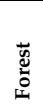 & đँ & $\dot{\Xi}$ \\
\hline Hieracium caespitosum & & nat & * & $\mathrm{x}$ & & & & aqc & \\
\hline Hieracium calodon & & nat & G & & & & & 226 & \\
\hline Hieracium cochleatum & & nat & $\mathrm{G}$ & & & & & dwn & \\
\hline Hieracium cymosum & & nat & 3 & $\mathrm{x}$ & & & & aqd & \\
\hline Hieracium densiflorum & & nat & G & & & & & cqo & \\
\hline Hieracium diaphanoides & & nat & $*$ & & & & & cqp & \\
\hline $\begin{array}{l}\text { Hieracium flagellare } \\
\text { lat }\end{array}$ & & nat & G & & & & & cqr & \\
\hline Hieracium flagelliferum & & nat & * & & & & & dps & \\
\hline Hieracium floribundum & & nat & G & & & & & cqs & \\
\hline Hieracium glaucinum & & nat & * & $\mathrm{x}$ & & & & aqe & \\
\hline Hieracium glaucisetigerum & & nat & G & & & & & 278 & \\
\hline Hieracium glomeratum & & nat & G & & & & & 279 & \\
\hline Hieracium guthnickianum & & nat & * & & & & & 7 & \\
\hline Hieracium kalksburgense & & nat & G & $\mathrm{x}$ & & & & 79 & \\
\hline Hieracium lachenalii & & nat & $*$ & $\mathrm{x}$ & & & & cqt & \\
\hline Hieracium lactucella & & nat & 2 & $\hat{x}$ & & & & aqf & \\
\hline Hieracium laevigatum & & nat & $*$ & $x$ & & & & cqu & \\
\hline Hieracium laurinum & & nat & * & & & & & cqv & \\
\hline Hieracium leptophyton & & nat & G & & & & & cqw & \\
\hline Hieracium longiscapum & & nat & $\mathrm{G}$ & & & & & dwm & \\
\hline Hieracium macrostolonum & & nat & * & & & & & 195 & \\
\hline Hieracium maculatum & & nat & G & $\mathrm{x}$ & & & & cqx & \\
\hline Hieracium murorum & & nat & * & & & & $\mathrm{x}$ & aqi & \\
\hline Hieracium pilosella & & nat & * & $\mathrm{x}$ & & & & cqy & \\
\hline Hieracium piloselloides & & nat & * & $\mathrm{x}$ & & & & $\mathrm{cqz}$ & \\
\hline Hieracium polymastix & & nat & * & & & & & 218 & \\
\hline Hieracium prussicum & & nat & G & & & & & 212 & \\
\hline Hieracium rothianum & & nat & $\mathrm{R}$ & $\mathrm{x}$ & & & & $\mathrm{cqq}$ & \\
\hline Hieracium sabaudum & & nat & * & $\hat{x}$ & & & & cra & \\
\hline Hieracium saxifragum & $\mathrm{x}$ & nat & $\mathrm{R}$ & & & & & 233 & \\
\hline Hieracium schmidtii & & nat & 1 & $\mathrm{x}$ & & & & aqg & \\
\hline Hieracium schultesii & $\mathrm{x}$ & nat & 1 & $\mathrm{x}$ & & & & $\mathrm{crb}$ & \\
\hline Hieracium stoloniflorum & & nat & * & & & & & 219 & \\
\hline Hieracium umbellatum & & nat & * & $\mathrm{x}$ & & & & crc & \\
\hline Hieracium visianii & & nat & * & & & & & 280 & \\
\hline Hieracium vulgatum & & nat & * & $\mathrm{x}$ & & & & crd & \\
\hline Hieracium zizianum & & nat & G & & & & & cre & \\
\hline Hierochloë odorata & & nat & 3 & $\mathrm{x}$ & & & & aqj & \\
\hline Hippocrepis comosa & & nat & 3 & $\mathrm{x}$ & & & & aqk & \\
\hline Hippophaë rhamnoides & $\mathrm{x}$ & nat & * & $\mathrm{x}$ & & & & $\mathrm{crf}$ & \\
\hline Hippuris vulgaris & & nat & 3 & & & & & aql & \\
\hline Holcus lanatus & & nat & * & & & & & crh & \\
\hline Holcus mollis & & nat & * & $\mathrm{x}$ & & & & cri & \\
\hline Holosteum umbellatum & & nat & $\mathrm{V}$ & $\mathrm{x}$ & & & & $\mathrm{aqm}$ & \\
\hline Honckenya peploides & & nat & * & & $\mathrm{x}$ & & & crj & \\
\hline Hordelymus europaeus & & nat & * & & & & $\mathrm{x}$ & crk & \\
\hline Hordeum jubatum & & neo & * & & & $\mathrm{x}$ & & $\mathrm{crl}$ & \\
\hline Hordeum murinum & & arc & * & & & $\mathrm{x}$ & & $\mathrm{crm}$ & \\
\hline Hordeum secalinum & & nat & $\mathrm{V}$ & & & & & aqo & \\
\hline Hornungia petraea & & nat & 1 & $\mathrm{x}$ & & & & aqp & \\
\hline Hottonia palustris & & nat & $\mathrm{V}$ & & & & & aqq & \\
\hline $\begin{array}{l}\text { Humulus lupulus } \\
\text { Hons }\end{array}$ & & nat & * & & $\mathrm{x}$ & & $\mathrm{x}$ & crn & \\
\hline Huperzia selago & & nat & 3 & & & & $\mathrm{x}$ & aqr & \\
\hline Hydrocharis morsus-ranae & & nat & $\mathrm{V}$ & & & & & aqs & \\
\hline Hydrocotyle vulgaris & & nat & * & $\mathrm{x}$ & & & & aqt & \\
\hline Hymenolobus procumbens & & neo & * & & & & & 36 & \\
\hline Hyoscyamus niger & & arc & 3 & & $\mathrm{x}$ & & & aqu & \\
\hline Hypericum elodes & & nat & 2 & $\mathrm{x}$ & & & & aqv & \\
\hline Hypericum hirsutum & & nat & * & & $\mathrm{x}$ & & $\mathrm{x}$ & aqw & \\
\hline Hypericum humifusum & & nat & * & $\mathrm{x}$ & & & & aqx & \\
\hline Hypericum maculatum & & nat & * & $\mathrm{x}$ & & & & crp & $\mathrm{ecm}$ \\
\hline Hypericum montanum & & nat & 3 & $\mathrm{x}$ & & & & aqy & \\
\hline Hypericum perforatum & & nat & * & & & & & crq & \\
\hline Hypericum pulchrum & & nat & * & $\mathrm{x}$ & & & & aqz & \\
\hline Hypericum tetrapterum & & nat & * & & & & & crr & \\
\hline Hypochaeris glabra & & nat & 2 & $\mathrm{x}$ & & & & ara & \\
\hline Hypochaeris maculata & & nat & 1 & $\mathrm{x}$ & & & & arb & \\
\hline Hypochaeris radicata & & nat & * & $\mathrm{x}$ & & & & crs & \\
\hline Ilex aquifolium & & nat & * & & & & $\mathrm{x}$ & crv & \\
\hline Illecebrum verticillatum & & nat & 3 & $\mathrm{x}$ & & & & arc & \\
\hline Impatiens glandulifera & & neo & * & & $\mathrm{x}$ & & & crw & \\
\hline Impatiens noli-tangere & & nat & * & & & & $\mathrm{x}$ & crx & \\
\hline Impatiens parviflora & & neo & * & & & & $\mathrm{x}$ & cry & \\
\hline
\end{tabular}


Table A.2 - Continued from previous page

\begin{tabular}{|c|c|c|c|c|c|c|c|c|c|}
\hline $\begin{array}{l}\mathscr{ٌ} \\
\text { ळूँ } \\
\text { के }\end{array}$ & 总 & 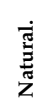 & $\begin{array}{l}\stackrel{\text { Z }}{a} \\
\underset{\approx}{2}\end{array}$ & $\begin{array}{l}\text { ? } \\
\mathbf{z} \\
z\end{array}$ & $\begin{array}{l}\stackrel{a}{a} \\
\mathbf{z} \\
\mathbb{z}\end{array}$ & 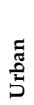 & 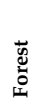 & نั & 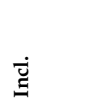 \\
\hline Inula britannica & & nat & 3 & & & & & ard & \\
\hline Inula conyzae & & nat & * & $\mathrm{x}$ & & & & $\mathrm{crz}$ & \\
\hline Inula germanica & $\mathrm{x}$ & nat & $\mathrm{R}$ & $\mathrm{x}$ & & & & are & \\
\hline Inula helenium & & neo & * & & & & & arf & \\
\hline Inula hirta & & nat & $\mathrm{R}$ & $\mathrm{x}$ & & & & $\arg$ & \\
\hline Inula salicina & & nat & 3 & $\mathrm{x}$ & & & & arh & \\
\hline Iris pseudacorus & & nat & * & & $\mathrm{x}$ & & & csa & \\
\hline Iris sibirica & & nat & 2 & $\mathrm{x}$ & & & & ari & \\
\hline Iris versicolor & & neo & * & & & & & $\mathrm{csb}$ & \\
\hline Isoëtes lacustris & & nat & 1 & $\mathrm{x}$ & & & & arj & \\
\hline Isolepis fluitans & & nat & 2 & $\mathrm{x}$ & & & & ark & \\
\hline Isolepis setacea & & nat & 3 & $\mathrm{x}$ & & & & arl & \\
\hline Jasione montana & & nat & * & $\mathrm{x}$ & & & & arm & \\
\hline Juncus acutiflorus & & nat & * & $\mathrm{x}$ & & & & cse & \\
\hline Juncus alpinus & & nat & 2 & $\mathrm{x}$ & & & & arn & \\
\hline Juncus anceps & $\mathrm{x}$ & nat & * & & & & & aro & \\
\hline Juncus articulatus & & nat & * & $\mathrm{x}$ & & & & $\operatorname{csf}$ & \\
\hline Juncus bufonius & & nat & * & & & & & $\operatorname{csg}$ & \\
\hline Juncus bulbosus & & nat & * & $\mathrm{x}$ & & & & $\operatorname{csh}$ & \\
\hline Juncus capitatus & & nat & 1 & $\mathrm{x}$ & & & & arq & \\
\hline Juncus compressus & & nat & * & & & & & $\mathrm{csi}$ & \\
\hline Juncus conglomeratus & & nat & * & $\mathrm{x}$ & & & & $\mathrm{csj}$ & \\
\hline Juncus effusus & & nat & * & & & & & csk & \\
\hline Juncus filiformis & & nat & 3 & $\mathrm{x}$ & & & & arr & \\
\hline Juncus gerardii & & nat & * & & & & & ars & \\
\hline Juncus inflexus & & nat & * & & & & & art & \\
\hline Juncus maritimus & $\mathrm{x}$ & nat & * & & & & & aru & \\
\hline Juncus minutulus & & nat & $\mathrm{D}$ & & & & & еср & \\
\hline Juncus ranarius & & nat & * & $\mathrm{x}$ & & & & $\mathrm{csm}$ & \\
\hline Juncus squarrosus & & nat & $\mathrm{V}$ & $\mathrm{x}$ & & & & $\operatorname{csn}$ & \\
\hline Juncus subnodulosus & & nat & 2 & $x$ & & & & arv & \\
\hline Juncus tenageia & & nat & 2 & & & & & arw & \\
\hline Juncus tenuis & & neo & * & & & & & cso & \\
\hline Juniperus communis & & nat & 3 & & & & & arx & \\
\hline Kalmia angustifolia & & neo & * & $\mathrm{x}$ & & $\mathrm{x}$ & & $\operatorname{csp}$ & \\
\hline Kickxia elatine & & arc & 2 & $\mathrm{x}$ & & & & ary & \\
\hline Kickxia spuria & & arc & 2 & $\mathrm{x}$ & & & & arz & \\
\hline Knautia arvensis & & nat & * & & & & & $\operatorname{csq}$ & \\
\hline Koeleria arenaria & $\mathrm{x}$ & nat & $\mathrm{V}$ & $\mathrm{x}$ & & & & asa & \\
\hline Koeleria glauca & & nat & 2 & $x$ & & & & asb & \\
\hline Koeleria macrantha & & nat & 3 & $\mathrm{x}$ & & & & asc & \\
\hline Koeleria pyramidata & & nat & $\mathrm{V}$ & $\mathrm{x}$ & & & & $\operatorname{css}$ & \\
\hline Lactuca serriola & & nat & * & & & $\mathrm{x}$ & & $\mathrm{csu}$ & \\
\hline Lactuca tatarica & & neo & * & & & & & $\operatorname{csv}$ & \\
\hline Lactuca virosa & & nat & 3 & & $\mathrm{x}$ & & & ase & \\
\hline Lamium album & & arc & * & & $x$ & & & $\operatorname{csw}$ & \\
\hline Lamium amplexicaule & & $\operatorname{arc}$ & * & & $\mathrm{x}$ & & & $\operatorname{cs} x$ & \\
\hline Lamium argentatum & & neo & * & & & $\mathrm{x}$ & & ecr & \\
\hline Lamium galeobdolon agg. & & nat & * & & & & & ecq & csz, cta \\
\hline Lamium maculatum & & nat & * & & $\mathrm{x}$ & & & $\mathrm{ctb}$ & \\
\hline Lamium purpureum & & $\operatorname{arc}$ & * & & $\mathrm{x}$ & & & ect & asf \\
\hline Lapsana communis & & nat & * & & $\mathrm{x}$ & & & ctd & \\
\hline Laser trilobum & & nat & 3 & $\mathrm{x}$ & & & $\mathrm{x}$ & ash & \\
\hline Laserpitium latifolium & & nat & 2 & $\mathrm{x}$ & & & & asi & \\
\hline Lathraea squamaria & & nat & $\mathrm{V}$ & & & & $\mathrm{x}$ & ask & \\
\hline Lathyrus hirsutus & & neo & * & & & & & $\operatorname{ctg}$ & \\
\hline Lathyrus latifolius & & neo & $*$ & $\mathrm{x}$ & & $x$ & & cth & \\
\hline Lathyrus linifolius & & nat & $\mathrm{V}$ & $\mathrm{x}$ & & & & asm & \\
\hline Lathyrus maritimus & $\mathrm{x}$ & nat & $\mathrm{R}$ & $\mathrm{x}$ & & & & asn & \\
\hline Lathyrus niger & & nat & 2 & $\mathrm{x}$ & & & $\mathrm{x}$ & aso & \\
\hline Lathyrus nissolia & & neo & * & & & & & cti & \\
\hline Lathyrus palustris & & nat & 2 & $\mathrm{x}$ & & & & asp & \\
\hline Lathyrus pratensis & & nat & * & & & & & $\mathrm{ctj}$ & \\
\hline Lathyrus sylvestris & & nat & * & $\mathrm{x}$ & & & & asq & \\
\hline Lathyrus tuberosus & & nat & $\mathrm{V}$ & & & & & asr & \\
\hline Lathyrus vernus & & nat & * & & & & $\mathrm{x}$ & ctl & \\
\hline Ledum palustre & & nat & 2 & $\mathrm{x}$ & & & & ass & \\
\hline Leersia oryzoides & & nat & 2 & & $\mathrm{x}$ & & & ast & \\
\hline Legousia hybrida & & arc & 2 & $\mathrm{x}$ & & & & asu & \\
\hline Legousia speculum-veneris & & $\operatorname{arc}$ & 1 & $\mathrm{x}$ & & & & asv & \\
\hline Lemna gibba & & nat & * & & $\mathrm{x}$ & & & $\mathrm{ctm}$ & \\
\hline Lemna minor & & nat & * & & & & & $\mathrm{ctn}$ & \\
\hline Lemna minuta & & neo & * & & & & & cto & \\
\hline
\end{tabular}


Table A.2 - Continued from previous page

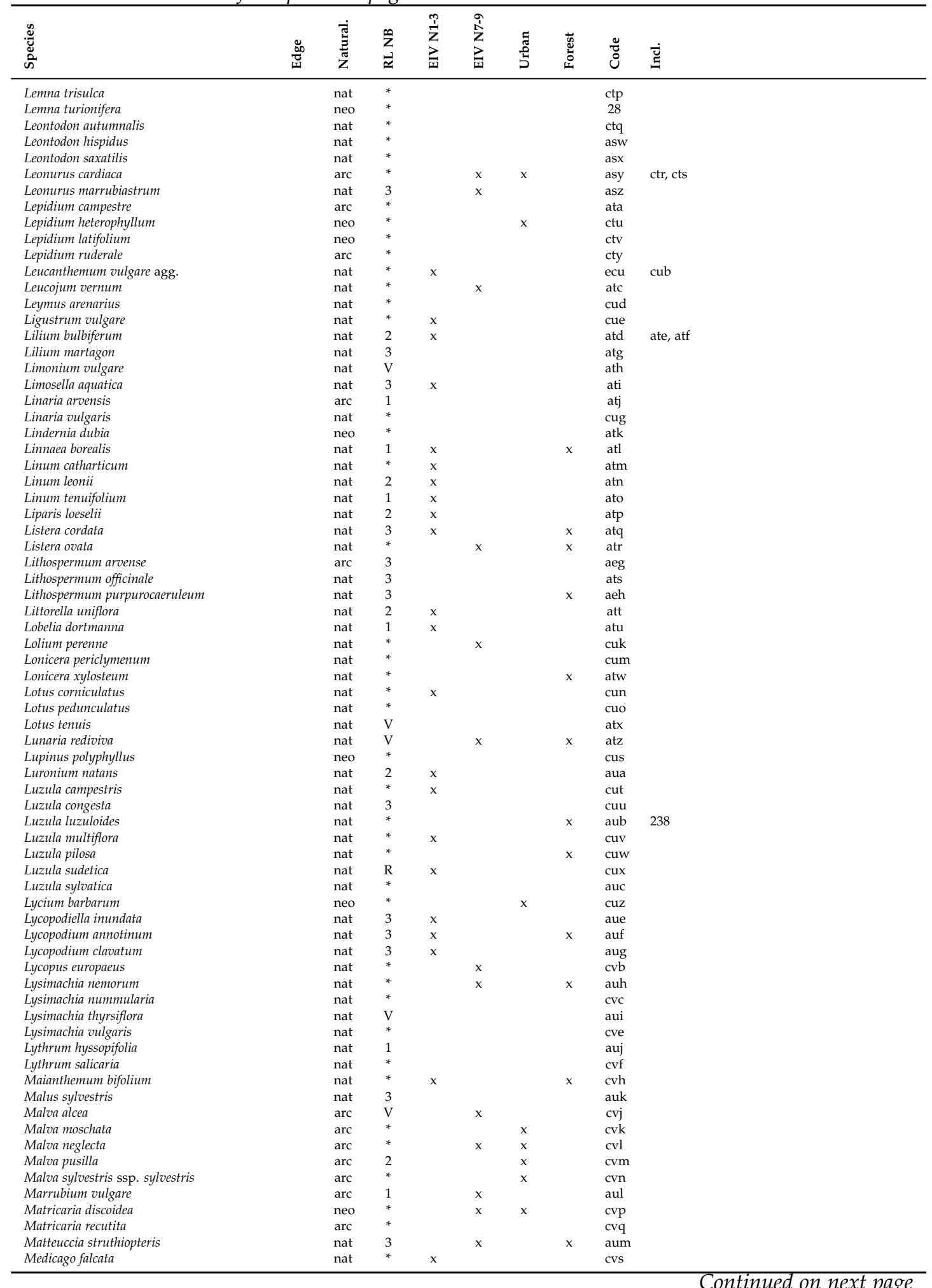


Table A.2 - Continued from previous page

\begin{tabular}{|c|c|c|c|c|c|c|c|c|c|}
\hline 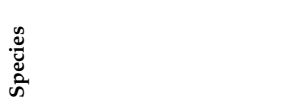 & 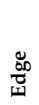 & 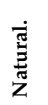 & $\begin{array}{l}\stackrel{\text { Z }}{a} \\
\underset{\approx}{2}\end{array}$ & $\begin{array}{l}\stackrel{?}{1} \\
\dot{z} \\
\text { 㭊 }\end{array}$ & $\begin{array}{l}\text { I } \\
\text { 乙 } \\
\text { 空 }\end{array}$ & $\begin{array}{l}\text { శ్ } \\
\text { 莡 } \\
\end{array}$ & 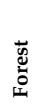 & نँ & $\begin{array}{l}\dot{\Xi} \\
\dot{g}\end{array}$ \\
\hline Medicago lupulina & & nat & * & & & & & cvt & \\
\hline Medicago minima & & nat & 2 & $\mathrm{x}$ & & & & aun & \\
\hline Medicago $\mathrm{x}$ varia & & neo & * & & & & & $\mathrm{cvu}$ & \\
\hline Melampyrum arvense & & nat & 2 & $\mathrm{x}$ & & & & auo & \\
\hline Melampyrum cristatum & & nat & 2 & $\mathrm{x}$ & & & $\mathrm{x}$ & aup & \\
\hline Melampyrum nemorosum & & nat & $\mathrm{V}$ & & & & $\mathrm{x}$ & auq & \\
\hline Melampyrum pratense & & nat & $*$ & $\mathrm{x}$ & & & & $\operatorname{cvv}$ & \\
\hline Melampyrum sylvaticum & & nat & * & $\mathrm{x}$ & & & & aur & \\
\hline Melica nutans & & nat & * & $\mathrm{x}$ & & & $\mathrm{x}$ & aus & \\
\hline Melica uniflora & & nat & * & & & & $\mathrm{x}$ & $\mathrm{cvw}$ & \\
\hline Melilotus albus & & $\operatorname{arc}$ & * & & & & & $\mathrm{cvx}$ & \\
\hline Melilotus altissimus & & nat & * & & $\mathrm{x}$ & & & cvy & \\
\hline Melilotus dentatus & & nat & 1 & & & & & aut & \\
\hline Melilotus officinalis & & $\operatorname{arc}$ & * & $\mathrm{x}$ & & & & cwa & \\
\hline Melittis melissophyllum & & nat & 1 & $\mathrm{x}$ & & & $\mathrm{x}$ & auu & \\
\hline Mentha aquatica & & nat & * & & & & & $\mathrm{cwb}$ & \\
\hline Mentha arvensis & & nat & * & & & & & cwc & \\
\hline Mentha longifolia & & nat & * & & $\mathrm{x}$ & & & auv & \\
\hline Mentha pulegium & & nat & 2 & & $\hat{x}$ & & & auw & \\
\hline Mentha $\times$ verticillata & & nat & * & & & & & $c w i$ & \\
\hline Menyanthes trifoliata & & nat & 3 & $\mathrm{x}$ & & & & aux & \\
\hline Mercurialis anпиа & & neo & * & & $\mathrm{x}$ & $\mathrm{x}$ & & $c w j$ & \\
\hline Mercurialis perennis & & nat & * & & $\mathrm{x}$ & & $\mathrm{x}$ & cwk & \\
\hline Meum athamanticum & & nat & $\mathrm{V}$ & $\mathrm{x}$ & & & & auy & \\
\hline Milium effusum & & nat & * & & & & $\mathrm{x}$ & cwl & \\
\hline Mimulus guttatus & & neo & * & & & & & auz & \\
\hline Mimulus moschatus & & neo & * & & & & & cwm & \\
\hline Minuartia verna ssp. hercynica & & nat & 3 & & & & & avb & \\
\hline Misopates orontium & & $\operatorname{arc}$ & 2 & & & & & avd & \\
\hline Moehringia trinervia & & nat & * & & $\mathrm{x}$ & & $\mathrm{x}$ & cwn & \\
\hline Molinia caerulea & & nat & * & $\mathrm{x}$ & & & & cwo & \\
\hline Moneses uniflora & & nat & 1 & $\mathrm{x}$ & & & $\mathrm{x}$ & avf & \\
\hline Monotropa hypopitys agg. & & nat & 3 & $\mathrm{x}$ & & & & avg & cwp, cwq \\
\hline Montia fontana & & nat & 3 & & & & & avh & cwi, avi, cws, cwt \\
\hline Mycelis muralis & & nat & * & & & & & $c w x$ & \\
\hline Myosotis arvensis & & arc & * & & & & & cwy & \\
\hline Myosotis discolor & & nat & $\mathrm{V}$ & $\mathrm{x}$ & & & & avj & \\
\hline Myosotis laxa & & nat & $*$ & & $x$ & & & avk & \\
\hline Myosotis nemorosa & & nat & * & & & & & avl & \\
\hline Myosotis ramosissima & & $\operatorname{arc}$ & * & $\mathrm{x}$ & & & & avm & \\
\hline Myosotis scorpioides & & nat & * & & & & & $\mathrm{cwz}$ & \\
\hline Myosotis sparsiflora & & nat & $\mathrm{R}$ & & $x$ & & & avn & \\
\hline Myosotis stricta & & $\operatorname{arc}$ & $\mathrm{V}$ & $\mathrm{x}$ & & & & cxa & \\
\hline Myosotis sylvatica & & nat & * & & $\mathrm{x}$ & & $\mathrm{x}$ & $\mathrm{cxb}$ & \\
\hline Myosurus minimus & & $\operatorname{arc}$ & * & & & & & avo & \\
\hline Myrica gale & & nat & 3 & $\mathrm{x}$ & & & & avp & \\
\hline Myriophyllum alterniflorum & & nat & 3 & $\hat{x}$ & & & & avq & \\
\hline Myriophyllum spicatum & & nat & * & & $\mathrm{x}$ & & & avr 1 & \\
\hline Myriophyllum verticillatum & & nat & * & & $\mathrm{x}$ & & & avs & \\
\hline Myrrhis odorata & & neo & * & & $\hat{x}$ & $\mathrm{x}$ & & cxc & \\
\hline Nardus stricta & & nat & $\mathrm{V}$ & $\mathrm{x}$ & & & & $\mathrm{cxf}$ & \\
\hline Narthecium ossifragum & & nat & 3 & $\mathrm{x}$ & & & & avu & \\
\hline Nasturtium officinale agg. & & nat & * & & $\mathrm{x}$ & & & edb & cxg, 191 \\
\hline Neottia nidus-avis & & nat & * & & & & $x$ & avv & \\
\hline Nepeta cataria & & $\operatorname{arc}$ & 2 & & $\mathrm{x}$ & $\mathrm{x}$ & & avw & \\
\hline Neslia paniculata & & arc & 1 & & & & & avx & \\
\hline Nonea pulla & & nat & 1 & $\mathrm{x}$ & & & & avz & \\
\hline Nuphar lutea & & nat & * & & & & & awa & \\
\hline Nuphar x spenneriana & & nat & $\mathrm{R}$ & & & & & $\mathrm{cxr}$ & \\
\hline Nymphaea alba & & nat & V & & & & & awc & \\
\hline Nymphaea candida & & nat & 3 & & & & & awd & \\
\hline Nymphoides peltata & & nat & 2 & & $\mathrm{x}$ & & & awe & \\
\hline Odontites litoralis & $\mathrm{x}$ & nat & 1 & & & & & awf & \\
\hline Odontites vernus & & nat & 3 & & & & & cxk & \\
\hline Odontites vulgaris & & nat & * & & & & & $\mathrm{cxl}$ & \\
\hline Oenanthe aquatica & & nat & * & & & & & $\mathrm{cxm}$ & \\
\hline Oenanthe conioides & $\mathrm{x}$ & nat & 1 & & $\mathrm{x}$ & & & awh & \\
\hline Oenanthe fistulosa & & nat & 3 & & & & & awi & \\
\hline Oenanthe lachenalii & & nat & 3 & & $\mathrm{x}$ & & & awj & \\
\hline Oenothera biennis & & neo & * & & & & & cxo & \\
\hline Oenothera oakesiana & & neo & * & & & & & cxn & \\
\hline Omphalodes scorpioides & & nat & 1 & & $\mathrm{x}$ & & $\mathrm{x}$ & awl & \\
\hline Onobrychis viciifolia & & neo & * & $\mathrm{x}$ & & & & cxs & \\
\hline
\end{tabular}


Table A.2 - Continued from previous page

\begin{tabular}{|c|c|c|c|c|c|c|c|c|c|}
\hline 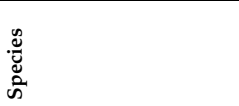 & $\underset{0}{\stackrel{0}{0}}$ & $\begin{array}{l}\dot{\Xi} \\
\frac{\Xi}{\pi} \\
\text { ż }\end{array}$ & 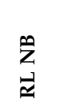 & $\begin{array}{l}\stackrel{p}{\dot{Z}} \\
\dot{z}\end{array}$ & $\begin{array}{l}a \\
\dot{a} \\
\mathbf{Z} \\
\vec{z}\end{array}$ & 䒕 & 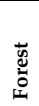 & ¿ँ & $\dot{\vec{\Xi}}$ \\
\hline Ononis spinosa agg. & & nat & * & & & & & awn & awm, awo \\
\hline Onopordum acanthium & & arc & * & & $\mathrm{x}$ & $\mathrm{x}$ & & awp & \\
\hline Ophioglossum vulgatum & & nat & 3 & $\mathrm{x}$ & & & & awg & \\
\hline Ophrys apifera & & nat & 3 & $\mathrm{x}$ & & & & awr & \\
\hline Ophrys insectifera & & nat & 3 & $\mathrm{x}$ & & & & aws & \\
\hline Orchis mascula & & nat & 3 & & & & & awu & \\
\hline Orchis militaris & & nat & 2 & $\mathrm{x}$ & & & & awv & \\
\hline Orchis purpurea & & nat & 3 & $\mathrm{x}$ & & & & awy & \\
\hline Orchis tridentata & & nat & 2 & $\mathrm{x}$ & & & & awz & \\
\hline Oreopteris limbosperma & & nat & * & & & & $\mathrm{x}$ & bgu & \\
\hline Origanum vulgare & & nat & * & $\mathrm{x}$ & & & & $\mathrm{axb}$ & \\
\hline Ornithogalum nutans agg. & & neo & * & & $\mathrm{x}$ & $\mathrm{x}$ & & axd & \\
\hline Ornithogalum umbellatum & & arc & * & & $\mathrm{x}$ & $\mathrm{x}$ & & axe & \\
\hline Ornithopus perpusillus & & nat & * & $\mathrm{x}$ & & & & cxw & \\
\hline Ornithopus sativus & & neo & * & & & & & $\mathrm{cxx}$ & \\
\hline Orobanche caryophyllacea & & nat & 1 & $\mathrm{x}$ & & & & axh & \\
\hline Orobanche elatior & & nat & 1 & $\mathrm{x}$ & & & & axi & \\
\hline Orobanche lutea & & nat & 1 & & & & & axj & \\
\hline Orobanche picridis & & nat & 1 & & & & & axm & \\
\hline Orobanche purpurea & & nat & $\begin{array}{l}1 \\
1\end{array}$ & $\mathrm{x}$ & & & & axn & \\
\hline Orobanche rapum-genistae & & nat & 2 & $\mathrm{x}$ & & & & $\operatorname{axp}$ & \\
\hline Orobanche reticulata & & nat & 3 & $\mathrm{x}$ & & & & axl & \\
\hline Orthilia secunda & & nat & 3 & $\hat{x}$ & & & $\mathrm{x}$ & axq & \\
\hline Osmunda regalis & & nat & 3 & & & & $\mathrm{x}$ & axr & \\
\hline Oxalis acetosella & & nat & * & & & & $\hat{x}$ & $c x z$ & \\
\hline Oxalis corniculata & & neo & * & & & $\mathrm{x}$ & & cya & \\
\hline Oxalis stricta & & neo & * & & $\mathrm{x}$ & & & cyc & \\
\hline Panicum riparium & & $\mathrm{n} / \mathrm{a}$ & * & & & & & dsf & \\
\hline Papaver argemone & & arc & * & & & & & cyh & \\
\hline Papaver dubium & & arc & * & & & & & ede & cyi, cyj \\
\hline Papaver rhoeas & & arc & * & & & & & axs & \\
\hline Parapholis strigosa & & nat & * & & & & & cyn & \\
\hline Parietaria judaica & & neo & * & & $\mathrm{x}$ & $\mathrm{x}$ & & axt & \\
\hline Parietaria officinalis & & arc & 3 & & $\hat{x}$ & $\hat{x}$ & & axu & \\
\hline Paris quadrifolia & & nat & V & & $\hat{x}$ & & $\mathrm{x}$ & axv & \\
\hline Parnassia palustris & & nat & 2 & $\mathrm{x}$ & & & & axw & \\
\hline Pastinaca sativa & & nat & * & & & & & cyr & \\
\hline Pedicularis palustris & & nat & 2 & $\mathrm{x}$ & & & & axx & \\
\hline Pedicularis sulvatica & & nat & 2 & $\mathrm{x}$ & & & & axy & \\
\hline Peplis portula & & nat & $\mathrm{V}$ & $\mathrm{x}$ & & & & axy & \\
\hline Persicaria amphibia & & nat & * & & & & & daf & \\
\hline Persicaria hydropiper & & nat & * & & $\mathrm{x}$ & & & dak & \\
\hline Persicaria lapathifolia & & nat & * & & & & & edl & dal, dam \\
\hline Persicaria maculosa & & nat & * & & $\mathrm{x}$ & & & dap & \\
\hline Persicaria minor & & nat & * & & $\mathrm{x}$ & & & dao & \\
\hline Persicaria mitis & & $\mathrm{n} / \mathrm{a}$ & * & & $\hat{x}$ & & & azh & \\
\hline Petasites albus & & nat & * & & & & & cys & \\
\hline Petasites hybridus & & nat & * & & $\mathrm{x}$ & & & cyt & \\
\hline Petasites spurius & & nat & 2 & & & & & aya & \\
\hline Petrorhagia prolifera & & nat & 2 & $\mathrm{x}$ & & & & $a y b$ & \\
\hline Peucedanum cervaria & & nat & 2 & $\hat{x}$ & & & & ayc & \\
\hline Peucedanum officinale & $\mathrm{x}$ & nat & 1 & $\hat{x}$ & & & & ayd & \\
\hline Peucedanum oreoselinum & & nat & 2 & $\mathrm{x}$ & & & & aye & \\
\hline Peucedanum ostruthium & & neo & $\mathrm{R}$ & & $\mathrm{x}$ & & & ayf & \\
\hline Peucedanum palustre & & nat & * & & & & & ayg & \\
\hline Phalaris arundinacea & & nat & * & & $\mathrm{x}$ & & & cyv & \\
\hline Phegopteris connectilis & & nat & * & & & & $\mathrm{x}$ & bgw & \\
\hline Phleum arenarium & $\mathrm{x}$ & nat & $\mathrm{V}$ & $\mathrm{x}$ & & & & ayh & \\
\hline Phleum bertolonii & & nat & * & & & & & cyx & \\
\hline Phleum paniculatum & $\mathrm{x}$ & nat & 1 & & & & & ayi & \\
\hline Phleum phleoides & & nat & 2 & $\mathrm{x}$ & & & & ayj & \\
\hline Phleum pratense & & nat & * & & $\mathrm{x}$ & & & cyy & \\
\hline Phragmites australis & & nat & * & & $\hat{x}$ & & & cyz & \\
\hline Physalis alkekengi & & nat & * & & $\hat{x}$ & & & ayl & \\
\hline Phyteuma nigrum & & nat & V & & & & $\mathrm{x}$ & aym & \\
\hline Phyteuma orbiculare & & nat & 2 & $\mathrm{x}$ & & & & ayn & \\
\hline Phyteuma spicatum & & nat & * & & & & $\mathrm{x}$ & ayo & $\mathrm{cza}, \mathrm{czb}$ \\
\hline Picea abies & & nat & * & & & & & $\mathrm{czc}$ & \\
\hline Picris hieracioides & & nat & * & & & & & cze & \\
\hline Pilularia globulifera & & nat & 2 & $\mathrm{x}$ & & & & ayp & \\
\hline Pimpinella major & & nat & $*$ & & & & & czf & \\
\hline Pimpinella nigra & & nat & $\mathrm{n} / \mathrm{a}$ & $\mathrm{x}$ & & & & 402 & \\
\hline Pimpinella saxifraga & & nat & $*$ & $\mathrm{x}$ & & & & $\mathrm{czg}$ & \\
\hline
\end{tabular}


Table A.2 - Continued from previous page

\begin{tabular}{|c|c|c|c|c|c|c|c|c|c|}
\hline $\begin{array}{l}\stackrel{\mathscr{J}}{\tilde{J}} \\
\text { के }\end{array}$ & $\underset{j}{\stackrel{0}{0}}$ & $\begin{array}{l}\text { 莺 } \\
\text { 䒿 }\end{array}$ & $\begin{array}{l}\stackrel{\text { Z }}{a} \\
\underset{\approx}{2}\end{array}$ & $\begin{array}{l}\text { ? } \\
\mathbf{z} \\
z\end{array}$ & $\begin{array}{l}\text { à } \\
\mathbf{z} \\
\text { 嵓 }\end{array}$ & 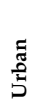 & 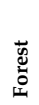 & نั & 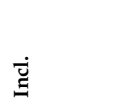 \\
\hline Pinguicula vulgaris & & nat & 2 & $\mathrm{x}$ & & & & ayq & \\
\hline Pinus sylvestris & & nat & * & & & & & $\mathrm{czi}$ & \\
\hline Plantago coronopus & & nat & * & & & & & ayr & \\
\hline Plantago lanceolata & & $\operatorname{arc}$ & * & & & & & czj & \\
\hline Plantago major & & nat & $*$ & & & & & edi & czk, czl, ayt \\
\hline Plantago maritima & & nat & * & & & & & ayu & \\
\hline Plantago media & & nat & * & $\mathrm{x}$ & & & & $\mathrm{czm}$ & \\
\hline Platanthera bifolia & & nat & 2 & & & & & ayv & \\
\hline Platanthera chlorantha & & nat & 3 & & & & $\mathrm{x}$ & ayw & \\
\hline Poa angustifolia & & nat & * & $\mathrm{x}$ & & & & czn & \\
\hline Роа аппиа & & nat & * & & $\mathrm{x}$ & & & czo & \\
\hline Poa bulbosa & & nat & 3 & $\mathrm{x}$ & & & & ayx & \\
\hline Poa chaixii & & nat & * & & & & $\mathrm{x}$ & czp & \\
\hline Poa compressa & & nat & * & $\mathrm{x}$ & & & & czq & \\
\hline Poa humilis & & nat & * & $\mathrm{x}$ & & & & $\mathrm{czu}$ & \\
\hline Poa nemoralis & & nat & * & & & & $\mathrm{x}$ & $\mathrm{czr}$ & \\
\hline Poa palustris & & nat & * & & $\mathrm{x}$ & & & $\mathrm{czs}$ & \\
\hline Poa pratensis & & nat & * & & & & & czt & \\
\hline Poa remota & & nat & 3 & & $\mathrm{x}$ & & $\mathrm{x}$ & ayy & \\
\hline Poa supina & & nat & * & & $\mathrm{x}$ & & & $\mathrm{czV}$ & \\
\hline Poa trivialis & & nat & * & & $\mathrm{x}$ & & & czw & \\
\hline Polemonium caeruleum & $\mathrm{x}$ & nat & $\mathrm{R}$ & & & & & czx & \\
\hline Polygala amara agg. & & nat & 2 & $\mathrm{x}$ & & & & azc & daa, dab \\
\hline Polygala comosa & & nat & $\mathrm{V}$ & $\mathrm{x}$ & & & & dac & \\
\hline Polygala serpyllifolia & & nat & 2 & $\mathrm{x}$ & & & & azd & \\
\hline Polygala vulgaris & & nat & 3 & $\mathrm{x}$ & & & & aze & cyk, cyq \\
\hline Polygonatum multiflorum & & nat & * & & & & $\mathrm{x}$ & dad & \\
\hline Polygonatum odoratum & & nat & 2 & $\mathrm{x}$ & & & & azf & \\
\hline Polygonatum verticillatum & & nat & * & & & & $\mathrm{x}$ & dae & \\
\hline Polygonum aviculare agg. & & nat & * & & & & & dag & 144 \\
\hline Polypodium vulgare agg. & & nat & * & $\mathrm{x}$ & & & & edm & dar, 276 \\
\hline Polystichum aculeatum & & nat & 3 & & $\mathrm{x}$ & & $\mathrm{x}$ & azi & \\
\hline Polystichum lonchitis & & nat & $\mathrm{R}$ & $\mathrm{x}$ & & & & azj & \\
\hline Populus alba & & neo & * & & & & & dat & \\
\hline Populus nigra & & nat & 3 & & $\mathrm{x}$ & & & azk & \\
\hline Populus tremula & & nat & * & & & & & dau & \\
\hline Populus $\mathrm{x}$ canescens & & neo & * & & & & & 258 & \\
\hline Portulaca oleracea & & neo & * & & $\mathrm{x}$ & $\mathrm{x}$ & & dav & \\
\hline Potamogeton acutifolius & & nat & 3 & & & & & azl & \\
\hline Potamogeton alpinus & & nat & $\mathrm{V}$ & & & & & azm & \\
\hline Potamogeton coloratus & & nat & 2 & & $\mathrm{x}$ & & & azo & \\
\hline Potamogeton compressus & & nat & 3 & & & & & azp & \\
\hline Potamogeton crispus & & nat & * & & & & & dax & \\
\hline Potamogeton friesii & & nat & 3 & & & & & azr & \\
\hline Potamogeton gramineus & & nat & 2 & & & & & azs & \\
\hline Potamogeton lucens & & nat & 3 & & $\mathrm{x}$ & & & azt & \\
\hline Potamogeton natans & & nat & * & & & & & day & \\
\hline Potamogeton nodosus & & nat & 3 & & & & & azu & \\
\hline Potamogeton obtusifolius & & nat & 3 & & & & & azv & \\
\hline Potamogeton pectinatus & & nat & * & & $\mathrm{x}$ & & & daz & \\
\hline Potamogeton perfoliatus & & nat & 3 & & & & & azw & \\
\hline Potamogeton polygonifolius & & nat & 3 & $\mathrm{x}$ & & & & azx & \\
\hline Potamogeton praelongus & & nat & 1 & & & & & azy & \\
\hline Potamogeton pusillus agg. & & nat & * & & & & & edo & daw \\
\hline Potamogeton trichoides & & nat & * & & & & & $\mathrm{dbb}$ & \\
\hline Potamogeton $\mathrm{x}$ angustifolius & & nat & 1 & & & & & azn & \\
\hline Potamogeton $\mathrm{x}$ nitens & & nat & 1 & & & & & dbe & \\
\hline Potamogeton $\times$ spathulatus & & nat & G & & & & & $\mathrm{dbg}$ & \\
\hline Potamogeton $\mathrm{x}$ undulatus & & $\mathrm{n} / \mathrm{a}$ & $\mathrm{R}$ & & & & & $\mathrm{dbh}$ & \\
\hline Potentilla alba & & nat & 1 & & & & & baa & \\
\hline Potentilla anglica & & nat & $\mathrm{V}$ & & & & & bab & \\
\hline Potentilla anserina & & nat & $*$ & & $\mathrm{x}$ & & & $\mathrm{dbi}$ & \\
\hline Potentilla argentea & & nat & * & $\mathrm{x}$ & & & & $\mathrm{dbj}$ & \\
\hline Potentilla erecta & & nat & * & $\mathrm{x}$ & & & & dbk & \\
\hline Potentilla heptaphylla & & nat & 2 & $x$ & & & & bae & \\
\hline Potentilla incana & & nat & 1 & $\mathrm{x}$ & & & & bac & \\
\hline Potentilla intermedia & & neo & * & & & $\mathrm{x}$ & & $\mathrm{dbm}$ & \\
\hline Potentilla neumanniana & & nat & V & $\mathrm{x}$ & & & & baf & \\
\hline Potentilla norvegica & & neo & $*$ & & & & & $\mathrm{dbn}$ & \\
\hline Potentilla palustris & & nat & $\mathrm{V}$ & $\mathrm{x}$ & & & & bag & \\
\hline Potentilla recta & & neo & * & $x$ & & & & dbo & \\
\hline Potentilla reptans & & nat & * & & & & & $\mathrm{dbp}$ & \\
\hline Potentilla sterilis & & nat & * & & & & $\mathrm{x}$ & bah & \\
\hline
\end{tabular}


Table A.2 - Continued from previous page

\begin{tabular}{|c|c|c|c|c|c|c|c|c|c|}
\hline 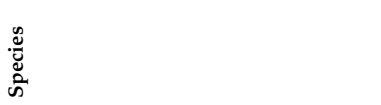 & 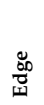 & 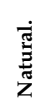 & 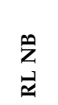 & $\begin{array}{l}\stackrel{p}{z} \\
\dot{z} \\
\dot{z}\end{array}$ & $\begin{array}{l}\text { ì } \\
\text { 之 } \\
\text { 空 }\end{array}$ & 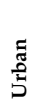 & 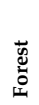 & ن & $\begin{array}{l}\dot{\Xi} \\
\text { E }\end{array}$ \\
\hline Potentilla supina & & nat & 3 & & $\mathrm{x}$ & & & bai & \\
\hline Primula elatior & & nat & * & & $\mathrm{x}$ & & $\mathrm{x}$ & baj & \\
\hline Primula veris & & nat & $\mathrm{V}$ & $\mathrm{x}$ & & & & bak & \\
\hline Primula vulgaris & & nat & 1 & & & & $\mathrm{x}$ & bal & \\
\hline Prunella grandiflora & & nat & 3 & $\mathrm{x}$ & & & & bam & \\
\hline Prunella laciniata & & nat & 2 & $\mathrm{x}$ & & & & ban & \\
\hline Prunella vulgaris & & nat & * & & & & & $\mathrm{dbr}$ & \\
\hline Prunus avium & & nat & * & & & & & dbs & \\
\hline Prunus padus & & nat & * & & & & & $d b x$ & \\
\hline Prunus serotina & & neo & * & & & & & dby & \\
\hline Prunus spinosa & & nat & * & & & & & $\mathrm{dbz}$ & \\
\hline Pseudognaphalium luteoalbum & & nat & 2 & $\mathrm{x}$ & & & & apf & \\
\hline Pseudolysimachion longifolium & & nat & 3 & $\lambda$ & & & & biw & \\
\hline Pseudolysimachion spicatum & & nat & 2 & $\mathrm{x}$ & & & & bjc & \\
\hline Pseudorchis albida & & nat & 1 & $\mathrm{x}$ & & & & bao & \\
\hline Psyllium arenarium & & neo & * & & & & & ays & \\
\hline Pteridium aquilinum & & nat & * & $\mathrm{x}$ & & & $\mathrm{x}$ & $\mathrm{dcb}$ & \\
\hline Puccinellia capillaris & $\mathrm{x}$ & nat & $\mathrm{R}$ & & & & & dcc & \\
\hline Puccinellia distans & & nat & * & & & & & dcd & \\
\hline Puccinellia limosa & & nat & $\mathrm{R}$ & & & & & 89 & \\
\hline Puccinellia maritima & & nat & * & & & & & dce & \\
\hline Pulicaria dysenterica & & nat & 3 & & & & & baq & \\
\hline Pulicaria vulgaris & & nat & 3 & & $\mathrm{x}$ & & & bar & \\
\hline Pulmonaria obscura & & nat & * & & $\mathrm{x}$ & & $\mathrm{x}$ & dcf & \\
\hline Pulmonaria officinalis & & nat & * & & & & $\mathrm{x}$ & bas & \\
\hline Pulsatilla pratensis & & nat & 2 & $\mathrm{x}$ & & & & bau & \\
\hline Pulsatilla vulgaris & & nat & 2 & $\mathrm{x}$ & & & & baw & \\
\hline Pyrola chlorantha & & nat & 1 & $\mathrm{x}$ & & & $\mathrm{x}$ & bax & \\
\hline Pyrola minor & & nat & 3 & $\mathrm{x}$ & & & $\mathrm{x}$ & baz & \\
\hline Pyrola rotundifolia & & nat & 3 & $\mathrm{x}$ & & & $\mathrm{x}$ & bba & \\
\hline Pyrus pyraster & & nat & 3 & & & & & $\mathrm{bbb}$ & \\
\hline Quercus petraea & & nat & * & & & & & dch & \\
\hline Quercus robur & & nat & * & & & & & dci & \\
\hline Quercus x rosacea & & nat & * & & & & & dsu & \\
\hline Radiola linoides & & nat & 3 & $\mathrm{x}$ & & & & $\mathrm{bbc}$ & \\
\hline Ranunculus acris & & nat & * & & & & & dcj & \\
\hline Ranunculus aquatilis agg. & & nat & * & & & & & bbd & bbe, bbi, bbj, bbk, bbn, bbg, bbo, dco, bbx \\
\hline Ranunculus arvensis & & arc & 2 & & & & & $\mathrm{bbf}$ & \\
\hline Ranunculus auricomus agg. & & nat & * & & & & & dck & \\
\hline Ranunculus bulbosus & & nat & * & $\mathrm{x}$ & & & & bbh & \\
\hline Ranunculus ficaria ssp. bulbilifer & & nat & * & & $\mathrm{x}$ & & & $\mathrm{dcl}$ & \\
\hline Ranunculus flammula & & nat & * & $\mathrm{x}$ & & & & $\mathrm{dcm}$ & \\
\hline Ranunculus lanuginosus & & nat & * & & $\mathrm{x}$ & & $\mathrm{x}$ & den & \\
\hline Ranunculus lingua & & nat & 3 & & $\mathrm{x}$ & & & $\mathrm{bbl}$ & \\
\hline Ranunculus platanifolius & & nat & 3 & & $\mathrm{x}$ & & & bbp & \\
\hline Ranunculus polyanthemos agg. & & nat & 3 & & & & & bbs & bbm, bbq, bbr, bbw, bbt \\
\hline Ranunculus repens & & nat & * & & $\mathrm{x}$ & & & dcp & \\
\hline Ranunculus sardous & & nat & 3 & & $\mathrm{x}$ & & & $\mathrm{bbv}$ & \\
\hline Ranunculus sceleratus & & nat & * & & $\mathrm{x}$ & & & dcq & \\
\hline Raphanus raphanistrum & & $\operatorname{arc}$ & 3 & & & & & der & \\
\hline Reseda lutea & & arc & * & & & & & dcu & \\
\hline Reseda luteola & & arc & * & & & & & dev & \\
\hline Rhamnus cathartica & & nat & * & & & & & bbz & \\
\hline Rhinanthus alectorolophus & & nat & 3 & & & & & bca & \\
\hline Rhinanthus angustifolius ssp. grandiflorus & & nat & $\mathrm{V}$ & $\mathrm{x}$ & & & & bcd & \\
\hline Rhinanthus glacialis & & nat & 1 & $\hat{x}$ & & & & $\mathrm{bcb}$ & \\
\hline Rhinanthus minor & & nat & $\mathrm{V}$ & $\mathrm{x}$ & & & & bcc & \\
\hline Rhynchospora alba & & nat & 3 & $x$ & & & & bce & \\
\hline Rhynchospora fusca & & nat & 2 & $\hat{x}$ & & & & bcf & \\
\hline Ribes alpinum & & nat & * & & $\mathrm{x}$ & & $\mathrm{x}$ & dew & \\
\hline Ribes nigrum & & nat & * & & & & $\mathrm{x}$ & $\mathrm{dcx}$ & \\
\hline Ribes rubrum agg. & & nat & * & & & & & edw & \\
\hline Ribes uva-crispa & & nat & * & & & $\mathrm{x}$ & & dda & \\
\hline Robinia pseudoacacia & & neo & * & & $\mathrm{x}$ & & & $\mathrm{ddb}$ & \\
\hline Rorippa amphibia & & nat & * & & $\hat{x}$ & & & ddc & \\
\hline Rorippa anceps & & nat & * & & $\mathrm{x}$ & & & bcg & \\
\hline Rorippa austriaca & & nat & $\mathrm{R}$ & & $x$ & & & bci & \\
\hline Rorippa palustris & & nat & * & & $\mathrm{x}$ & & & ddd & \\
\hline Rorippa sylvestris & & nat & * & & & & & dde & \\
\hline Rosa agrestis & & nat & 2 & $\mathrm{x}$ & & & & $\mathrm{bcj}$ & \\
\hline Rosa arvensis & & nat & 2 & & & & $\mathrm{x}$ & bck & \\
\hline Rosa caesia agg. & & nat & $\mathrm{n} / \mathrm{a}$ & & & & & $\mathrm{bcl}$ & $\mathrm{ddf}$ \\
\hline Rosa canina & & nat & * & & & & & $\mathrm{ddg}$ & \\
\hline
\end{tabular}


Table A.2 - Continued from previous page

\begin{tabular}{|c|c|c|c|c|c|c|c|c|c|}
\hline $\begin{array}{l}\mathscr{ٌ} \\
\text { ळूँ } \\
\text { के }\end{array}$ & $\underset{j}{\stackrel{0}{0}}$ & 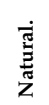 & $\begin{array}{l}\stackrel{\text { Z }}{a} \\
\underset{\approx}{2}\end{array}$ & $\begin{array}{l}p \\
\dot{z} \\
z \\
z\end{array}$ & $\begin{array}{l}\text { à } \\
\mathbf{z} \\
\text { 嵓 }\end{array}$ & 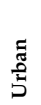 & 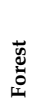 & రั & 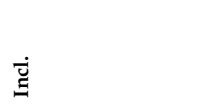 \\
\hline Rosa corymbifera & & nat & * & & & & & $\mathrm{ddh}$ & \\
\hline Rosa dumalis & & nat & 3 & & & & & dds & bcq \\
\hline Rosa elliptica & & nat & 3 & $\mathrm{x}$ & & & & $\mathrm{bcm}$ & \\
\hline Rosa inodora & & nat & G & & & & & 87 & \\
\hline Rosa micrantha & & nat & 3 & $\mathrm{x}$ & & & & ddj & \\
\hline Rosa rubiginosa & & nat & * & $\mathrm{x}$ & & & & ddk & \\
\hline Rosa rugosa & & neo & * & & & & & ddl & \\
\hline Rosa spinosissima & $\mathrm{x}$ & nat & 3 & $\mathrm{x}$ & & & & bco & \\
\hline Rosa subcanina & & nat & * & $\mathrm{x}$ & & & & ddo & \\
\hline Rosa subcollina & & nat & 3 & $\mathrm{x}$ & & & & $\mathrm{ddp}$ & \\
\hline Rosa tomentella & & nat & 3 & & & & & bcn & \\
\hline Rosa tomentosa agg. & & nat & 3 & & & & & bcp & $\mathrm{ddm}, \mathrm{ddn}, \mathrm{ddq}, \mathrm{ddr}$ \\
\hline Rubus armeniacus & & neo & * & & & $\mathrm{x}$ & & dea & \\
\hline Rubus caesius & & nat & * & & $\mathrm{x}$ & & & $\operatorname{deg}$ & \\
\hline Rubus chamaemorus & & nat & 2 & $\mathrm{x}$ & & & & bcr & \\
\hline Rubus fruticosus agg. & & nat & * & & & & & edy & \\
\hline Rubus idaeus & & nat & * & & & & $\mathrm{x}$ & dgi & \\
\hline Rubus laciniatus & & neo & * & & & & & dgo & \\
\hline Rubus saxatilis & & nat & 2 & & & & $\mathrm{x}$ & bcs & \\
\hline Rumex acetosa & & nat & * & & & & & djt & \\
\hline Rumex acetosella & & nat & * & $\mathrm{x}$ & & & & dju & \\
\hline Rumex aquaticus & & nat & $\mathrm{V}$ & & $\mathrm{x}$ & & & bct & \\
\hline Rumex conglomeratus & & nat & * & & $\mathrm{x}$ & & & djv & \\
\hline Rumex crispus & & nat & * & & & & & djw & \\
\hline Rumex hydrolapathum & & nat & * & & $\mathrm{x}$ & & & djx & \\
\hline Rumex maritimus & & nat & * & & $\mathrm{x}$ & & & $\mathrm{bcu}$ & \\
\hline Rumex obtusifolius & & nat & * & & $\mathrm{x}$ & & & eeb & \\
\hline Rumex palustris & & nat & * & & $\mathrm{x}$ & & & $\mathrm{bcv}$ & \\
\hline Rumex salicifolius & $\mathrm{x}$ & neo & * & & $\mathrm{x}$ & & & dke & \\
\hline Rumex sanguineus & & nat & * & & $\mathrm{x}$ & & $\mathrm{x}$ & dka & \\
\hline Rumex stenophyllus & & neo & * & & & & & $\mathrm{dkc}$ & \\
\hline Rumex thyrsiflorus & & nat & * & & & & & $\mathrm{dkd}$ & \\
\hline Rumex $\mathrm{x}$ pratensis & & $\mathrm{n} / \mathrm{a}$ & * & & & & & far & \\
\hline Ruppia cirrhosa & $\mathrm{x}$ & nat & 2 & & & & & $\mathrm{dkf}$ & \\
\hline Ruppia maritima & $\mathrm{x}$ & nat & 2 & & & & & bcw & \\
\hline Sagina apetala agg. & & arc & * & & & & & eed & $\mathrm{dkh}$ \\
\hline Sagina maritima & & nat & * & $\mathrm{x}$ & & & & dki & \\
\hline Sagina nodosa & & nat & 3 & & & & & bcx & \\
\hline Sagina procumbens & & nat & * & & & $\mathrm{x}$ & & $\mathrm{dkj}$ & \\
\hline Sagittaria latifolia & & neo & * & & & & & $\mathrm{dkk}$ & \\
\hline Sagittaria sagittifolia & & nat & $*$ & & & & & bcz & \\
\hline Salicornia dolichostachya s.l. & & nat & $\mathrm{n} / \mathrm{a}$ & $\mathrm{x}$ & & & & eef & \\
\hline Salicornia europaea & & nat & $*$ & & & & & eee & bda, dkn \\
\hline Salicornia fragilis & $\mathrm{x}$ & nat & $\mathrm{n} / \mathrm{a}$ & & & & & dko & \\
\hline Salicornia procumbens & & nat & $*$ & $\mathrm{x}$ & & & & $\mathrm{dkl}$ & \\
\hline Salicornia stricta & & nat & * & & & & & $\mathrm{dkm}$ & \\
\hline Salix alba & & nat & * & & $\mathrm{x}$ & & & eeg & $\mathrm{dkq}$ \\
\hline Salix aurita & & nat & * & $\mathrm{x}$ & & & & $\mathrm{dkr}$ & \\
\hline Salix caprea & & nat & * & & $\mathrm{x}$ & & & dks & \\
\hline Salix cinerea & & nat & * & & & & & $\mathrm{dkt}$ & \\
\hline Salix fragilis agg. & & nat & * & & & & & eeh & $\mathrm{dkv}$, dle \\
\hline Salix pentandra & & nat & 3 & & & & & $\mathrm{dkw}$ & \\
\hline Salix purpurea & & nat & * & & & & & eei & dky \\
\hline Salix repens & & nat & * & & & & & eej & dla, bdd \\
\hline Salix triandra & & nat & * & & & & & eek & $\mathrm{dlb}$ \\
\hline Salix viminalis & & nat & * & & & & & dld & \\
\hline Salix $\mathrm{x}$ alopecuroides & & nat & * & & & & & 101 & \\
\hline Salix $\times$ ambigua & & nat & * & & & & & $\mathrm{drc}$ & \\
\hline Salix $x$ holosericea & & nat & * & & & & & $\mathrm{drb}$ & \\
\hline Salix $\mathrm{x}$ meyeriana & & nat & * & & & & & dqr & \\
\hline Salix $\mathrm{x}$ mollissima & & nat & * & & & & & dqi & \\
\hline Salix $\times$ multinervis & & nat & * & & & & & $\mathrm{dkx}$ & \\
\hline Salix $\mathrm{x}$ reichardtii & & nat & * & & & & & dqh & \\
\hline Salix $\times$ rubra & & nat & * & & & & & dqf & \\
\hline Salix $\mathrm{x}$ smithiana & & nat & * & & & & & dlf & \\
\hline Salsola kali & & nat & * & & & & & bdf & $\mathrm{dlg}, \mathrm{dlh}$ \\
\hline Salvia glutinosa & & neo & * & & $\mathrm{x}$ & & & dli & \\
\hline Salvia nemorosa & & neo & * & & & & & $\mathrm{dlj}$ & \\
\hline Salvia pratensis & & nat & 3 & & & & & bdg & \\
\hline Salvia verticillata & & neo & * & & & & $\mathrm{x}$ & bdh & \\
\hline Sambucus ebulus & & neo & * & & $\mathrm{x}$ & $\mathrm{x}$ & $\mathrm{x}$ & dll & \\
\hline Sambucus nigra & & nat & * & & $x$ & & & $\mathrm{dlm}$ & 14 \\
\hline Sambucus racemosa & & nat & * & & $\mathrm{x}$ & & & $d \ln$ & \\
\hline
\end{tabular}


Table A.2 - Continued from previous page

\begin{tabular}{|c|c|c|c|c|c|c|c|c|c|}
\hline $\begin{array}{l}. \stackrel{\mathscr{U}}{.} \\
\text { के }\end{array}$ & 总 & 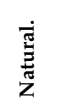 & $\begin{array}{l}\stackrel{\text { Z }}{\beth} \\
\vec{z}\end{array}$ & $\begin{array}{l}m \\
\dot{1} \\
z \\
z\end{array}$ & $\begin{array}{l}a \\
\stackrel{a}{z} \\
z\end{array}$ & 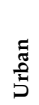 & 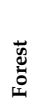 & نँ & $\dot{\vec{\Xi}}$ \\
\hline Samolus valerandi & & nat & 2 & & & & & bdi & \\
\hline Sanguisorba minor & & nat & * & $\mathrm{x}$ & & & & eel & bdj \\
\hline Sanguisorba officinalis & & nat & 3 & & & & & bdk & \\
\hline Sanicula europaea & & nat & * & & & & & bdl & \\
\hline Saponaria officinalis & & nat & * & & & $\mathrm{x}$ & & dlp & \\
\hline Saxifraga granulata & & nat & 3 & $\mathrm{x}$ & & & & bdm & \\
\hline Saxifraga tridactylites & & nat & * & $\mathrm{x}$ & & & & bdo & \\
\hline Scabiosa canescens & & nat & 2 & $\mathrm{x}$ & & & & bdp & \\
\hline Scabiosa columbaria & & nat & * & $\mathrm{x}$ & & & & $\mathrm{bdq}$ & \\
\hline Scandix pecten-veneris & & arc & 2 & & & & & bds & \\
\hline Scheuchzeria palustris & & nat & 2 & $\mathrm{x}$ & & & & bdt & \\
\hline Schoenoplectus lacustris & & nat & * & & & & & dlt & \\
\hline Schoenoplectus pungens & & nat & 1 & & $\mathrm{x}$ & & & bdu & \\
\hline Schoenoplectus tabernaemontani & & nat & * & & & & & bdw & \\
\hline Schoenoplectus triqueter & & nat & 3 & & $\mathrm{x}$ & & & $\mathrm{bdx}$ & \\
\hline Schoenoplectus $\mathrm{x}$ carinatus & & nat & 3 & & $\mathrm{x}$ & & & bdy & \\
\hline Schoenus nigricans & $\mathrm{x}$ & nat & 3 & $\mathrm{x}$ & & & & bdz & \\
\hline Scirpus sylvaticus & & nat & * & & & & & dlu & \\
\hline Scleranthus annuus agg. & & arc & * & & & & & een & een \\
\hline Scleranthus perennis & & nat & 3 & $\mathrm{x}$ & & & & bea & \\
\hline Scorzonera hispanica & $\mathrm{x}$ & nat & 2 & $\mathrm{x}$ & & & & beb & \\
\hline Scorzonera humilis & & nat & 2 & $\mathrm{x}$ & & & & bec & \\
\hline Scorzonera laciniata & & nat & 2 & & & & & ayz & \\
\hline Scrophularia nodosa & & nat & * & & $\mathrm{x}$ & & & dly & \\
\hline Scrophularia umbrosa & & nat & * & & $\mathrm{x}$ & & & bee & \\
\hline Scrophularia vernalis & & neo & * & & $\hat{x}$ & $\mathrm{x}$ & & $\mathrm{dlz}$ & \\
\hline Scutellaria galericulata & & nat & * & & & & & dma & \\
\hline Scutellaria hastifolia & & nat & 2 & & & & & bef & \\
\hline Scutellaria minor & & nat & 2 & $\mathrm{x}$ & & & & beg & \\
\hline Securigera varia & & neo & * & $\mathrm{x}$ & & & & ajo & \\
\hline Sedum acre & & nat & * & $\mathrm{x}$ & & & & $\mathrm{dmb}$ & \\
\hline Sedum album & & nat & * & $x$ & & & & beh & \\
\hline Sedum rupestre & & nat & $\mathrm{V}$ & $\mathrm{x}$ & & & & bei & \\
\hline Sedum sexangulare & & nat & $*$ & $\mathrm{x}$ & & & & bej & \\
\hline Sedum telephium agg. & & nat & * & & & & & eeo & dmd, dme \\
\hline Selinum carvifolia & & nat & 3 & $\mathrm{x}$ & & & & bel & \\
\hline Sempervivum tectorum & & nat & * & & & & & bem & \\
\hline Senecio aquaticus & & nat & 3 & & & & & ben & \\
\hline Senecio erraticus ssp. barbareifolius & & nat & 3 & & & & & bep & \\
\hline Senecio erucifolius & & nat & * & & & & & $\mathrm{dmg}$ & \\
\hline Senecio hercynicus & & nat & * & & $\mathrm{x}$ & & $\mathrm{x}$ & bes & \\
\hline Senecio inaequidens & & neo & * & $\mathrm{x}$ & & $\mathrm{x}$ & & $\mathrm{dmh}$ & \\
\hline Senecio jacobaea ssp. dunensis & $\mathrm{x}$ & nat & * & & & & & $\mathrm{dmi}$ & \\
\hline Senecio jacobaea ssp. jacobaea & & nat & * & & & & & $\mathrm{dmj}$ & \\
\hline Senecio ovatus & & nat & * & & $\mathrm{x}$ & & $\mathrm{x}$ & dmk & \\
\hline Senecio paludosus & & nat & 2 & & & & & bet & \\
\hline Senecio sarracenicus & & nat & * & & $\mathrm{x}$ & & & beq & \\
\hline Senecio sylvaticus & & nat & * & & $\mathrm{x}$ & & $\mathrm{x}$ & $\mathrm{dml}$ & \\
\hline Senecio vernalis & & neo & * & & & & & $\mathrm{dmm}$ & \\
\hline Senecio viscosus & & nat & * & & & & & $\mathrm{dmn}$ & \\
\hline Senecio vulgaris & & nat & * & & $\mathrm{x}$ & & & dmo & \\
\hline Serratula tinctoria & & nat & 2 & $\mathrm{x}$ & & & & beu & \\
\hline Seseli anпиит & & nat & 1 & $\mathrm{x}$ & & & & bev & \\
\hline Seseli libanotis & & nat & 2 & $\mathrm{x}$ & & & & bew & \\
\hline Seseli montanum & & nat & 1 & & & & & $\mathrm{dmp}$ & \\
\hline Sesleria albicans & & nat & V & $\mathrm{x}$ & & & & bex & \\
\hline Setaria pumila & & arc & $\mathrm{V}$ & & & & & bey & \\
\hline Setaria viridis & & arc & * & & $\mathrm{x}$ & & & $\mathrm{dmt}$ & \\
\hline Sherardia arvensis & & arc & 3 & & & & & bez & \\
\hline Silaum silaus & & nat & 2 & $\mathrm{x}$ & & & & bfa & \\
\hline Silene conica & $\mathrm{x}$ & neo & * & $\mathrm{x}$ & & & & $\mathrm{dmw}$ & \\
\hline Silene dioica & & nat & * & & $\mathrm{x}$ & & & $\mathrm{dmx}$ & \\
\hline Silene flos-cuculi & & nat & * & & & & & cuy & \\
\hline Silene latifolia ssp. alba & & nat & * & & $\mathrm{x}$ & & & $\mathrm{dmz}$ & \\
\hline Silene noctiflora & & arc & 3 & & & & & bfc & \\
\hline Silene nutans & & nat & $\mathrm{V}$ & $\mathrm{x}$ & & & & bfd & \\
\hline Silene otites & & nat & 3 & $x$ & & & & bfe & \\
\hline Silene viscaria & & nat & 1 & $\mathrm{x}$ & & & & aud & \\
\hline Silene vulgaris & & nat & * & & & & & bff & \\
\hline Silene $\mathrm{x}$ hampeana & & $\mathrm{n} / \mathrm{a}$ & * & & & & & dqw & \\
\hline Sinapis arvensis & & arc & * & & & & & dnd & \\
\hline Sisymbrium altissimum & & neo & * & & & & & dne & \\
\hline Sisymbrium austriacum & & nat & $\mathrm{R}$ & & $\mathrm{x}$ & & & bfg & \\
\hline
\end{tabular}


Table A.2 - Continued from previous page

\begin{tabular}{|c|c|c|c|c|c|c|c|c|c|}
\hline $\begin{array}{l}\stackrel{\mathscr{J}}{\tilde{J}} \\
\text { के }\end{array}$ & $\underset{j}{\stackrel{0}{0}}$ & 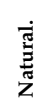 & $\begin{array}{l}\vec{Z} \\
\vec{z}\end{array}$ & $\begin{array}{l}p \\
\dot{z} \\
z \\
z\end{array}$ & $\begin{array}{l}\stackrel{a}{a} \\
\mathbf{z} \\
\mathbb{z}\end{array}$ & 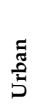 & 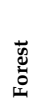 & نั & 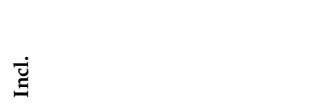 \\
\hline Sisymbrium loeselii & & neo & * & & & & & dng & \\
\hline Sisymbrium officinale & & $\operatorname{arc}$ & * & & $\mathrm{x}$ & & & dnh & \\
\hline Sisymbrium strictissimum & & nat & $\mathrm{R}$ & & $\mathrm{x}$ & & & bfh & \\
\hline Sium latifolium & & nat & * & & $\mathrm{x}$ & & & bfi & \\
\hline Solanum dulcamara & & nat & * & & $\mathrm{x}$ & & & dnk & \\
\hline Solanum nigrum & & arc & * & & $\mathrm{x}$ & & & eeq & $\mathrm{dnm}$ \\
\hline Solidago canadensis & & neo & * & & & & & dns & \\
\hline Solidago gigantea & & neo & * & & $\mathrm{x}$ & & & dnt & \\
\hline Solidago virgaurea & & nat & * & & & & & dnv & \\
\hline Sonchus arvensis & & nat & * & & & & & eer & dnw, bfk \\
\hline Sonchus asper & & nat & * & & $\mathrm{x}$ & & & $\operatorname{dn} x$ & \\
\hline Sonchus oleraceus & & nat & * & & $\mathrm{x}$ & & & dny & \\
\hline Sonchus palustris & & nat & $\mathrm{V}$ & & $\mathrm{x}$ & & & $\mathrm{bfl}$ & \\
\hline Sorbus aucuparia & & nat & * & & & & & $\operatorname{dnz}$ & \\
\hline Sorbus torminalis & & nat & * & & & & & bfo & \\
\hline Sparganium angustifolium & & nat & 2 & $\mathrm{x}$ & & & & bfp & \\
\hline Sparganium emersum & & nat & * & & $\mathrm{x}$ & & & doa & \\
\hline Sparganium erectum & & nat & * & & $\mathrm{x}$ & & & ees & doc \\
\hline Sparganium natans & & nat & 2 & $\mathrm{x}$ & & & & $\mathrm{bfq}$ & \\
\hline Spartina anglica & & neo & * & $\mathrm{x}$ & & & & dod & \\
\hline Spergula arvensis & & $\operatorname{arc}$ & * & & & & & doe & \\
\hline Spergula morisonii & & nat & * & $\mathrm{x}$ & & & & dof & \\
\hline Spergularia echinosperma & & nat & * & & $\mathrm{x}$ & & & bfr & \\
\hline Spergularia media & & nat & * & & & & & $\operatorname{dog}$ & \\
\hline Spergularia rubra & & $\operatorname{arc}$ & * & & & & & doh & \\
\hline Spergularia salina & & nat & * & & & & & bfs & \\
\hline Spiraea alba & & neo & * & & & & & $\mathrm{dpf}$ & \\
\hline Spiranthes spiralis & & nat & 1 & $\mathrm{x}$ & & & & bfu & \\
\hline Spirodela polyrhiza & & nat & * & & & & & dok & \\
\hline Stachys alpina & & nat & 3 & & $\mathrm{x}$ & & $\mathrm{x}$ & $\mathrm{bfv}$ & \\
\hline Stachys aппиа & & arc & 1 & & & & & $\mathrm{bfw}$ & \\
\hline Stachys arvensis & & $\operatorname{arc}$ & 3 & & & & & bfx & \\
\hline Stachys germanica & & nat & 2 & & & & & bfy & \\
\hline Stachys palustris & & nat & $*$ & & & & & dol & \\
\hline Stachys recta & & nat & 2 & $\mathrm{x}$ & & & & bfz & \\
\hline Stachys sylvatica & & nat & * & & $\mathrm{x}$ & & $\mathrm{x}$ & dom & \\
\hline Stachys $\mathrm{x}$ ambigua & & nat & * & & & & & 103 & \\
\hline Stellaria alsine & & nat & * & & & & & $\operatorname{dot}$ & \\
\hline Stellaria aquatica & & nat & * & & $\mathrm{x}$ & & & don & \\
\hline Stellaria graminea & & nat & * & $\mathrm{x}$ & & & & doo & \\
\hline Stellaria holostea & & nat & * & & & & $\mathrm{x}$ & dop & \\
\hline Stellaria media & & $\operatorname{arc}$ & * & & & & & doq & \\
\hline Stellaria neglecta & & nat & * & & & & $\mathrm{x}$ & dor & \\
\hline Stellaria nemorum & & nat & * & & $\mathrm{x}$ & & & $\mathrm{bgb}$ & \\
\hline Stellaria pallida & & arc & * & & & & & dos & \\
\hline Stellaria palustris & & nat & $\mathrm{V}$ & $\mathrm{x}$ & & & & $\mathrm{bgc}$ & \\
\hline Stipa capillata & $\mathrm{x}$ & nat & $\mathrm{R}$ & $x$ & & & & bgd & \\
\hline Stratiotes aloides & & nat & 3 & & & & & bge & \\
\hline Suaeda maritima & & nat & * & & $\mathrm{x}$ & & & dou & \\
\hline Succisa pratensis & & nat & 3 & $\mathrm{x}$ & & & & dov & \\
\hline Symphytum officinale & & nat & * & & $\mathrm{x}$ & & & doy & \\
\hline Symphytum $\times$ uplandicum & & neo & * & & & & & doz & \\
\hline Tanacetum corymbosum & & nat & 3 & & & & $\mathrm{x}$ & bgh & \\
\hline Tanacetum vulgare & & arc & * & & & $\mathrm{x}$ & & $\mathrm{dpc}$ & \\
\hline Taraxacum acervatulum & & nat & $\mathrm{n} / \mathrm{a}$ & & & & & 104 & \\
\hline Taraxacum acutifrons & & nat & $\mathrm{n} / \mathrm{a}$ & & & & & dpe & \\
\hline Taraxacum adiantifrons & & nat & $\mathrm{n} / \mathrm{a}$ & & & & & dqn & \\
\hline Taraxacum aequilobum & & nat & $\mathrm{n} / \mathrm{a}$ & & & & & 106 & \\
\hline Taraxacum alatum & & nat & $\mathrm{n} / \mathrm{a}$ & & & & & $\mathrm{dph}$ & \\
\hline Taraxacum amphilobum & & nat & $\mathrm{n} / \mathrm{a}$ & & & & & 107 & \\
\hline Taraxacum amplum & & nat & $\mathrm{n} / \mathrm{a}$ & & & & & dpi & \\
\hline Taraxacum ancistrolobum & & nat & $\mathrm{n} / \mathrm{a}$ & & & & & dpj & \\
\hline Taraxacum angustisquameum & & nat & $\mathrm{n} / \mathrm{a}$ & & & & & 108 & \\
\hline Taraxacum atactum & & nat & $\mathrm{n} / \mathrm{a}$ & & & & & dpk & \\
\hline Taraxacum atricapillum & $\mathrm{x}$ & nat & $\mathrm{n} / \mathrm{a}$ & & & & & 110 & \\
\hline Taraxacum baeckiiforme & & nat & $\mathrm{n} / \mathrm{a}$ & & & & & dpn & \\
\hline Taraxacum borgvallii & & nat & $\mathrm{n} / \mathrm{a}$ & & & & & dpp & \\
\hline Taraxacum cacuminatum & & nat & $\mathrm{n} / \mathrm{a}$ & & & & & 111 & \\
\hline Taraxacum calochroum & $\mathrm{x}$ & nat & $\mathrm{n} / \mathrm{a}$ & & & & & 112 & \\
\hline Taraxacum caloschistum & & nat & $\mathrm{n} / \mathrm{a}$ & & & & & dpu & \\
\hline Taraxacum canoviride & & nat & $\mathrm{n} / \mathrm{a}$ & & & & & 113 & \\
\hline Taraxacum celticum agg. & & nat & $\mathrm{V}$ & & & & & eex & dpr, dsa, 26, 283, drg, 181, 182, dqj \\
\hline Taraxacum chlorodes & $\mathrm{x}$ & nat & $\mathrm{n} / \mathrm{a}$ & & & & & 114 & \\
\hline
\end{tabular}


Table A.2 - Continued from previous page

\begin{tabular}{|c|c|c|c|c|c|c|c|c|c|}
\hline 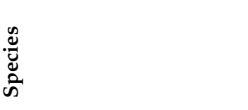 & 萡 & 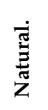 & $\begin{array}{l}\tilde{Z} \\
\stackrel{a}{\simeq}\end{array}$ & $\begin{array}{l}\dot{p} \\
\dot{z} \\
\dot{z}\end{array}$ & $\begin{array}{l}\stackrel{a}{\hat{i}} \\
\mathbf{z} \\
z_{1}\end{array}$ & 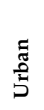 & 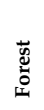 & نั & $\dot{\vec{\Xi}}$ \\
\hline Taraxacum christiansenii & $\mathrm{x}$ & nat & $\mathrm{n} / \mathrm{a}$ & & & & & 115 & \\
\hline Taraxacum comtulum & & nat & $\mathrm{n} / \mathrm{a}$ & & & & & 117 & \\
\hline Taraxacum contractum & & nat & $\mathrm{n} / \mathrm{a}$ & & & & & 119 & \\
\hline Taraxacum copidophyllum & & nat & $\mathrm{n} / \mathrm{a}$ & & & & & 120 & \\
\hline Taraxacum cordatum & & nat & $\mathrm{n} / \mathrm{a}$ & & & & & dpw & \\
\hline Taraxacum croceiflorum & $\mathrm{x}$ & nat & $\mathrm{n} / \mathrm{a}$ & & & & & $\mathrm{dpz}$ & \\
\hline Taraxacum cyanolepis & & nat & $\mathrm{n} / \mathrm{a}$ & & & & & dqa & \\
\hline Taraxacum dilaceratum & & nat & $\mathrm{n} / \mathrm{a}$ & & & & & dqb & \\
\hline Taraxacum dilatatum & $\mathrm{x}$ & nat & $\mathrm{n} / \mathrm{a}$ & & & & & 121 & \\
\hline Taraxacum edytomum & & nat & $\mathrm{n} / \mathrm{a}$ & & & & & 140 & \\
\hline Taraxacum ekmanii & & nat & $\mathrm{n} / \mathrm{a}$ & & & & & dqd & \\
\hline Taraxacum exsertiforme & & nat & $\mathrm{n} / \mathrm{a}$ & & & & & 123 & \\
\hline Taraxacum exsertum & & nat & $\mathrm{n} / \mathrm{a}$ & & & & & 124 & \\
\hline Taraxacum fagerstroemii & & nat & $\mathrm{n} / \mathrm{a}$ & & & & & 125 & \\
\hline Taraxacum fasciatum & & nat & $\mathrm{n} / \mathrm{a}$ & & & & & dqg & \\
\hline Taraxacum fulgidum & & nat & $\mathrm{n} / \mathrm{a}$ & & & & & 127 & \\
\hline Taraxacum fusciflorum & & nat & $\mathrm{n} / \mathrm{a}$ & & & & & 57 & \\
\hline Taraxacum gelertiiforme & & nat & $\mathrm{n} / \mathrm{a}$ & & & & & 128 & \\
\hline Taraxacum gesticulans & & nat & $\mathrm{n} / \mathrm{a}$ & & & & & 129 & \\
\hline Taraxacum hamatiforme & & nat & $\mathrm{n} / \mathrm{a}$ & & & & & $\mathrm{dqk}$ & \\
\hline Taraxacum hamatulum & & nat & $\mathrm{n} / \mathrm{a}$ & & & & & 58 & \\
\hline Taraxacum hamatum & & nat & $\mathrm{n} / \mathrm{a}$ & & & & & 130 & \\
\hline Taraxacum hamatum agg. & & nat & * & & & & & $\mathrm{dql}$ & \\
\hline Taraxacum hamiferum & $\mathrm{x}$ & nat & $\mathrm{n} / \mathrm{a}$ & & & & & dqm & \\
\hline Taraxacum hepaticum & & nat & $\mathrm{n} / \mathrm{a}$ & & & & & 131 & \\
\hline Taraxacum huelphersianum & & nat & $\mathrm{n} / \mathrm{a}$ & & & & & dqo & \\
\hline Taraxacum inarmatum & & nat & $\mathrm{n} / \mathrm{a}$ & & & & & 132 & \\
\hline Taraxacum ingens & & nat & $\mathrm{n} / \mathrm{a}$ & & & & & dqq & \\
\hline Taraxacum inops & & nat & $\mathrm{n} / \mathrm{a}$ & & & & & 133 & \\
\hline Taraxacum interveniens & & nat & $\mathrm{n} / \mathrm{a}$ & & & & & 134 & \\
\hline Taraxacum intumescens & & nat & $\mathrm{n} / \mathrm{a}$ & & & & & 135 & \\
\hline Taraxacum kernianum & & nat & $\mathrm{n} / \mathrm{a}$ & & & & & dqs & \\
\hline Taraxacum laciniosifrons & & nat & $\mathrm{n} / \mathrm{a}$ & & & & & dqt & \\
\hline Taraxacum laciniosum & $\mathrm{x}$ & nat & $\mathrm{n} / \mathrm{a}$ & & & & & dqu & \\
\hline Taraxacum lacinulatum & & nat & $\mathrm{n} / \mathrm{a}$ & & & & & 136 & \\
\hline Taraxacum laevigatum agg. & & nat & * & $\mathrm{x}$ & & & & bgi & $\begin{array}{l}\text { dpq, } 116,122,56,126 \text {, dqv, dsg, drw, 34, dsd, } \\
\text { dsr, dss }\end{array}$ \\
\hline Taraxacum lamprophyllum & & nat & $\mathrm{n} / \mathrm{a}$ & & & & & dqx & \\
\hline Taraxacum latens & & nat & $\mathrm{n} / \mathrm{a}$ & & & & & 137 & \\
\hline Taraxacum laticordatum & & nat & $\mathrm{n} / \mathrm{a}$ & & & & & dqy & \\
\hline Taraxacum latisectum & $\mathrm{x}$ & nat & $\mathrm{n} / \mathrm{a}$ & & & & & 138 & \\
\hline Taraxacum latissimum & & nat & $\mathrm{n} / \mathrm{a}$ & & & & & 139 & \\
\hline Taraxacum leptodon & & nat & $\mathrm{n} / \mathrm{a}$ & & & & & $\mathrm{dqz}$ & \\
\hline Taraxacum lingulatum & & nat & $\mathrm{n} / \mathrm{a}$ & & & & & 142 & \\
\hline Taraxacum lojoense & & nat & $\mathrm{n} / \mathrm{a}$ & & & & & 143 & \\
\hline Taraxacum lucidum & & nat & $\mathrm{n} / \mathrm{a}$ & & & & & drd & \\
\hline Taraxacum macranthoides & $\mathrm{x}$ & nat & $\mathrm{n} / \mathrm{a}$ & & & & & 145 & \\
\hline Taraxacum maculatum & & nat & $\mathrm{n} / \mathrm{a}$ & & & & & 147 & \\
\hline Taraxacum melanostigma & & nat & $\mathrm{n} / \mathrm{a}$ & & & & & 148 & \\
\hline Taraxacum melanthoides & & nat & $\mathrm{n} / \mathrm{a}$ & & & & & dre & \\
\hline Taraxacum mimulum & & nat & $\mathrm{n} / \mathrm{a}$ & & & & & drf & \\
\hline Taraxacum necessarium & & nat & $\mathrm{n} / \mathrm{a}$ & & & & & 149 & \\
\hline Taraxacum nitidum & & nat & $\mathrm{n} / \mathrm{a}$ & & & & & 150 & \\
\hline Taraxacum obliquilobum & & nat & $\mathrm{n} / \mathrm{a}$ & & & & & drh & \\
\hline Taraxacum obliquum & $\mathrm{x}$ & nat & $\mathrm{R}$ & & & & & dri & \\
\hline Taraxacum oblongatum & & nat & $\mathrm{n} / \mathrm{a}$ & & & & & drj & \\
\hline Taraxacum obtusifrons & & nat & $\mathrm{n} / \mathrm{a}$ & & & & & drk & \\
\hline Taraxacum ochrochlorum & & nat & $\mathrm{n} / \mathrm{a}$ & & & & & 151 & \\
\hline Taraxacum officinale agg. & & nat & * & & $\mathrm{x}$ & & & eey & \\
\hline Taraxacum ostenfeldii & & nat & $\mathrm{n} / \mathrm{a}$ & & & & & dqc & \\
\hline Taraxacum oxyrhinum & & nat & $\mathrm{n} / \mathrm{a}$ & & & & & 152 & \\
\hline Taraxacum pachymerum & $\mathrm{x}$ & nat & $\mathrm{n} / \mathrm{a}$ & & & & & 153 & \\
\hline Taraxacum pallidipes & & nat & $\mathrm{n} / \mathrm{a}$ & & & & & drm & \\
\hline Taraxacum palustre agg. & & nat & 3 & $\mathrm{x}$ & & & & bgj & drn, 59, dpo, 40, 27 \\
\hline Taraxacum pannисіum & & nat & $\mathrm{n} / \mathrm{a}$ & & & & & dro & \\
\hline Taraxacum pannulatum & & nat & $\mathrm{n} / \mathrm{a}$ & & & & & 159 & \\
\hline Taraxacum pectinatiforme & & nat & $\mathrm{n} / \mathrm{a}$ & & & & & $\mathrm{drq}$ & \\
\hline Taraxacum piceatum & & nat & $\mathrm{n} / \mathrm{a}$ & & & & & drr & \\
\hline Taraxacum piceipictum & & nat & $\mathrm{n} / \mathrm{a}$ & & & & & 160 & \\
\hline Taraxacum planum & & nat & $\mathrm{n} / \mathrm{a}$ & & & & & 105 & \\
\hline Taraxacum plicatifrons & & nat & $\mathrm{n} / \mathrm{a}$ & & & & & 161 & \\
\hline Taraxacum polyodon & & nat & $\mathrm{n} / \mathrm{a}$ & & & & & drt & \\
\hline Taraxacum porrigens & & nat & $\mathrm{n} / \mathrm{a}$ & & & & & 162 & \\
\hline
\end{tabular}


Table A.2 - Continued from previous page

\begin{tabular}{|c|c|c|c|c|c|c|c|c|c|}
\hline 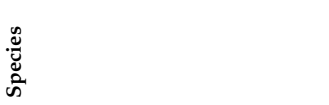 & 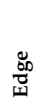 & 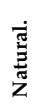 & $\begin{array}{l}\stackrel{\infty}{z} \\
\stackrel{z}{\approx}\end{array}$ & $\begin{array}{l}\stackrel{?}{1} \\
\dot{z} \\
\text { 㭊 }\end{array}$ & $\begin{array}{l}\stackrel{a}{1} \\
\text { z } \\
\text { zir }\end{array}$ & 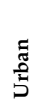 & 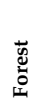 & نँ & $\begin{array}{l}\dot{\vec{\Xi}} \\
\dot{3}\end{array}$ \\
\hline Taraxacum privum & & nat & $\mathrm{n} / \mathrm{a}$ & & & & & dru & \\
\hline Taraxacum pruinatum & & nat & $\mathrm{n} / \mathrm{a}$ & & & & & 183 & \\
\hline Taraxacum pseudoretroflexum & & nat & $\mathrm{n} / \mathrm{a}$ & & & & & 163 & \\
\hline Taraxacum pulcherrimum & & nat & $\mathrm{n} / \mathrm{a}$ & & & & & 164 & \\
\hline Taraxacum pulchrifolium & & nat & $\mathrm{n} / \mathrm{a}$ & & & & & drx & \\
\hline Taraxacum pulverulentum & $\mathrm{x}$ & nat & $\mathrm{n} / \mathrm{a}$ & & & & & 156 & \\
\hline Taraxacum purpureum & & nat & $\mathrm{n} / \mathrm{a}$ & & & & & drs & \\
\hline Taraxacum quadrans & & nat & $\mathrm{n} / \mathrm{a}$ & & & & & drz & \\
\hline Taraxacum rhodopodum & & nat & $\mathrm{n} / \mathrm{a}$ & & & & & 165 & \\
\hline Taraxacum rigens & & nat & $\mathrm{n} / \mathrm{a}$ & & & & & 166 & \\
\hline Taraxacum sagittipotens & & nat & $\mathrm{n} / \mathrm{a}$ & & & & & dsc & \\
\hline Taraxacum sellandii & & nat & $\mathrm{n} / \mathrm{a}$ & & & & & 157 & \\
\hline Taraxacum semiglobosum & & nat & $\mathrm{n} / \mathrm{a}$ & & & & & 167 & \\
\hline Taraxacum severum & & nat & $\mathrm{n} / \mathrm{a}$ & & & & & 168 & \\
\hline Taraxacum sinuatum & & nat & $\mathrm{n} / \mathrm{a}$ & & & & & dsh & \\
\hline Taraxacum stereodes & & nat & $\mathrm{n} / \mathrm{a}$ & & & & & $\mathrm{dsj}$ & \\
\hline Taraxacum subcanescens & & nat & $\mathrm{n} / \mathrm{a}$ & & & & & 169 & \\
\hline Taraxacum subdahlstedtii & & nat & $\mathrm{n} / \mathrm{a}$ & & & & & 170 & \\
\hline Taraxacum subditivum & & nat & $\mathrm{n} / \mathrm{a}$ & & & & & 184 & \\
\hline Taraxacum subericinum & & nat & $\mathrm{n} / \mathrm{a}$ & & & & & 185 & \\
\hline Taraxacum subhamatum & & nat & $\mathrm{n} / \mathrm{a}$ & & & & & dsk & \\
\hline Taraxacum subhuelphersianum & & nat & $\mathrm{n} / \mathrm{a}$ & & & & & 171 & \\
\hline Taraxacum sublaeticolor & $\mathrm{x}$ & nat & $\mathrm{n} / \mathrm{a}$ & & & & & dsl & \\
\hline Taraxacum subleucopodum & & nat & $\mathrm{n} / \mathrm{a}$ & & & & & 172 & \\
\hline Taraxacum subserratifrons & & nat & $\mathrm{n} / \mathrm{a}$ & & & & & 173 & \\
\hline Taraxacum subundulatum & & nat & $\mathrm{n} / \mathrm{a}$ & & & & & dso & \\
\hline Taraxacum subxanthostigma & $\mathrm{x}$ & nat & $\mathrm{n} / \mathrm{a}$ & & & & & 174 & \\
\hline Taraxacum tenebricans & & nat & $\mathrm{n} / \mathrm{a}$ & & & & & dsq & \\
\hline Taraxacum tumentilobum & & nat & $\mathrm{n} / \mathrm{a}$ & & & & & dsv & \\
\hline Taraxacum undulatiflorum & & nat & $\mathrm{n} / \mathrm{a}$ & & & & & 175 & \\
\hline Taraxacum undulatiforme & $\mathrm{x}$ & nat & $\mathrm{n} / \mathrm{a}$ & & & & & 176 & \\
\hline Taraxacum undulatum & & nat & $\mathrm{n} / \mathrm{a}$ & & & & & 177 & \\
\hline Taraxacum unguifrons & $\mathrm{x}$ & nat & $\mathrm{n} / \mathrm{a}$ & & & & & 178 & \\
\hline Taraxacum valens & & nat & $\mathrm{n} / \mathrm{a}$ & & & & & 154 & \\
\hline Taraxacum vanum & & nat & $\mathrm{n} / \mathrm{a}$ & & & & & 179 & \\
\hline Taraxacum violaceinervosum & & nat & $\mathrm{n} / \mathrm{a}$ & & & & & 158 & \\
\hline Taxus baccata & & nat & 3 & & & & & bgk & \\
\hline Teesdalia nudicaulis & & nat & * & $\mathrm{x}$ & & & & bgl & \\
\hline Tephroseris palustris & & nat & 2 & & $\mathrm{x}$ & & & beo & \\
\hline Tetragonolobus maritimus & & nat & 1 & $\mathrm{x}$ & & & & bgm & \\
\hline Teucrium botrys & & arc & 2 & $\hat{x}$ & & & & bgn & \\
\hline Teucrium scordium & & nat & 2 & & & & & bgo & \\
\hline Teucrium scorodonia & & nat & * & $\mathrm{x}$ & & & $\mathrm{x}$ & bgp & \\
\hline Thalictrum flavum & & nat & 3 & & & & & bgq & \\
\hline Thalictrum lucidum & & nat & 3 & $\mathrm{x}$ & & & & bgr & \\
\hline Thalictrum minus ssp. minus & & nat & 2 & & & & & bgs & \\
\hline Thalictrum simplex ssp. tenuifolium & & nat & $\mathrm{R}$ & & & & & bgt & \\
\hline Thelypteris palustris & & nat & 3 & & & & & bgv & \\
\hline Thesium ebracteatum & & nat & 1 & $\mathrm{x}$ & & & & bgx & \\
\hline Thesium linophyllon & & nat & 1 & $\hat{x}$ & & & & bgy & \\
\hline Thesium pyrenaicum & & nat & 2 & $\mathrm{x}$ & & & & bgz & \\
\hline Thlaspi arvense & & $\operatorname{arc}$ & * & & & & & dta & \\
\hline Thlaspi caerulescens & & nat & 1 & & & & & $\mathrm{dtb}$ & \\
\hline Thlaspi calaminare & $\mathrm{x}$ & nat & 1 & $\mathrm{x}$ & & & & bha & \\
\hline Thlaspi perfoliatum & & nat & $\mathrm{V}$ & $\mathrm{x}$ & & & & bhb & \\
\hline Thymus praecox & & nat & $\mathrm{R}$ & $\hat{x}$ & & & & bhe & \\
\hline Thymus pulegioides & & nat & $*$ & $\mathrm{x}$ & & & & $\mathrm{dtc}$ & \\
\hline Thymus serpyllum & & nat & 3 & $x$ & & & & $\mathrm{dtd}$ & \\
\hline Tilia cordata & & nat & * & & & & & dte & \\
\hline Tilia platyphyllos & & nat & * & & $\mathrm{x}$ & & & $\mathrm{dtf}$ & \\
\hline Torilis japonica & & nat & * & & $\mathrm{x}$ & & $\mathrm{x}$ & $\mathrm{dtg}$ & \\
\hline Torilis nodosa & & neo & 3 & & & & & bhe & \\
\hline Tragopogon dubius & & nat & * & & & & & bhf & \\
\hline Tragopogon pratensis ssp. minor & & nat & $\mathrm{D}$ & & & & & dth & \\
\hline Tragopogon pratensis ssp. orientalis & & nat & $\mathrm{R}$ & & & & & $\mathrm{dti}$ & \\
\hline Tragopogon pratensis ssp. pratensis & & nat & $*$ & & & & & $\mathrm{dtj}$ & \\
\hline Trichomanes speciosum & & nat & $\mathrm{R}$ & & & & $x$ & 95 & \\
\hline Trichophorum cespitosum & & nat & 3 & & & & & bhg & 260, bhh, bhi \\
\hline Trientalis europaea & & nat & * & $\mathrm{x}$ & & & $\mathrm{x}$ & $\mathrm{dtk}$ & \\
\hline Trifolium alpestre & & nat & 2 & $x$ & & & & bhj & \\
\hline Trifolium arvense & & nat & * & $\mathrm{x}$ & & & & $\mathrm{dtm}$ & \\
\hline Trifolium aureum & & nat & 3 & $\mathrm{x}$ & & & & bhk & \\
\hline Trifolium campestre & & nat & * & $\hat{x}$ & & & & dtn & \\
\hline
\end{tabular}


Table A.2 - Continued from previous page

\begin{tabular}{|c|c|c|c|c|c|c|c|c|c|}
\hline 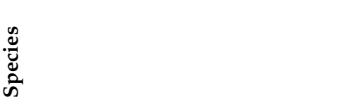 & 总 & 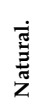 & $\begin{array}{l}\tilde{z} \\
\stackrel{a}{\approx}\end{array}$ & $\begin{array}{l}\stackrel{p}{\vec{z}} \\
\dot{z}\end{array}$ & $\begin{array}{l}a \\
\stackrel{a}{z} \\
z\end{array}$ & 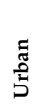 & 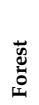 & نँ & $\begin{array}{l}\dot{\Xi} \\
\dot{\Xi}\end{array}$ \\
\hline Trifolium dubium & & nat & * & & & & & dto & \\
\hline Trifolium fragiferum & & nat & * & & $\mathrm{x}$ & & & bhl & \\
\hline Trifolium hybridum & & neo & * & & & & & dtp & \\
\hline Trifolium medium & & nat & * & $\mathrm{x}$ & & & & bhm & \\
\hline Trifolium montanum & & nat & 3 & $\mathrm{x}$ & & & & bhn & \\
\hline Trifolium pratense & & nat & * & & & & & dts & \\
\hline Trifolium repens & & nat & * & & & & & $\mathrm{dtt}$ & \\
\hline Trifolium spadiceum & $\mathrm{x}$ & nat & 2 & $\mathrm{x}$ & & & & bhg & \\
\hline Trifolium striatum & & nat & 2 & $\mathrm{x}$ & & & & bhr & \\
\hline Triglochin maritimum & & nat & * & & & & & bhs & \\
\hline Triglochin palustre & & nat & 3 & $\mathrm{x}$ & & & & bht & \\
\hline Tripleurospermum perforatum & & $\operatorname{arc}$ & * & & & & & $\mathrm{dtw}$ & \\
\hline Trisetum flavescens & & nat & * & & & & & $\mathrm{dtx}$ & \\
\hline Trollius europaeus & & nat & 2 & & & & & bhu & \\
\hline Tuberaria guttata & $\mathrm{x}$ & nat & $\mathrm{R}$ & $\mathrm{x}$ & & & & bhv & \\
\hline Tulipa sylvestris & & arc & 3 & & & $\mathrm{x}$ & & bhw & \\
\hline Tussilago farfara & & nat & * & & & & & dty & \\
\hline Typha angustifolia & & nat & * & & $\mathrm{x}$ & & & $\mathrm{dtz}$ & \\
\hline Typha latifolia & & nat & * & & $\mathrm{x}$ & & & dua & \\
\hline Ulex europaeus & & neo & 3 & $\mathrm{x}$ & & & & bhy & \\
\hline Ulmus glabra & & nat & * & & $\mathrm{x}$ & & & dub & \\
\hline Ulmus laevis & & nat & 3 & & $\mathrm{x}$ & & & bhz & \\
\hline Ulmus minor & & nat & 3 & & & & & duc & \\
\hline Urtica dioica ssp. dioica & & nat & * & & & & & dud & \\
\hline Urtica urens & & $\operatorname{arc}$ & * & & $\mathrm{x}$ & $\mathrm{x}$ & & due & \\
\hline Utricularia australis & $\mathrm{x}$ & nat & 3 & $\mathrm{x}$ & & & & bia & \\
\hline Utricularia intermedia & & nat & 1 & $\mathrm{x}$ & & & & bib & \\
\hline Utricularia minor & & nat & 3 & $\mathrm{x}$ & & & & bic & \\
\hline Utricularia vulgaris agg. & & nat & 3 & & & & & bie & bif \\
\hline Vaccinium angustifolium $\times$ corymbosum & & neo & * & & & & & eaa & \\
\hline Vaccinium macrocarpon & & neo & * & $\mathrm{x}$ & & & & duf & \\
\hline Vaccinium myrtillus & & nat & * & $\mathrm{x}$ & & & & dug & \\
\hline Vaccinium oxycoccos & & nat & 3 & $\mathrm{x}$ & & & & bii ${ }^{\circ}$ & \\
\hline Vaccinium uliginosum & & nat & 3 & $\mathrm{x}$ & & & & bij & \\
\hline Vaccinium vitis-idaea & & nat & * & $\mathrm{x}$ & & & & bik & \\
\hline Vaccinium $\mathrm{x}$ intermedium & & nat & * & & & & & bih & \\
\hline Valeriana dioica & & nat & $\mathrm{V}$ & $\mathrm{x}$ & & & & bil & \\
\hline Valeriana officinalis agg. & & nat & * & & & & & efc & bim, bin \\
\hline Valerianella carinata & & nat & * & & & & & bio & \\
\hline Valerianella dentata & & nat & 3 & & & & & bip & \\
\hline Valerianella locusta & & arc & * & & & & & duj & \\
\hline Valerianella rimosa & & nat & 2 & & & & & biq & \\
\hline Verbascum blattaria & & neo & $\mathrm{R}$ & & & & & duk & \\
\hline Verbascum densiflorum & & nat & * & & & & & dul & \\
\hline Verbascum lychnitis & & nat & * & $\mathrm{x}$ & & & & bir & \\
\hline Verbascum nigrum & & nat & * & & $\mathrm{x}$ & & & dum & \\
\hline Verbascum phlomoides & & nat & * & & & & & dun & \\
\hline Verbascum phoeniceum & & nat & 1 & $\mathrm{x}$ & & $\mathrm{x}$ & & bis & \\
\hline Verbascum thapsus & & nat & * & & $\mathrm{x}$ & & & bit & \\
\hline Verbena officinalis & & arc & $\mathrm{V}$ & & $\hat{x}$ & $x$ & & biu & \\
\hline Veronica agrestis & & arc & $\mathrm{V}$ & & $\mathrm{x}$ & & & dup & \\
\hline Veronica anagallis-aquatica agg. & & nat & * & & & & & biv & duu \\
\hline Veronica arvensis & & arc & * & & & & & dus & \\
\hline Veronica beccabunga & & nat & * & & & & & dut & \\
\hline Veronica chamaedrys & & nat & * & & & & & duv & \\
\hline Veronica filiformis & & neo & * & & $\mathrm{x}$ & $\mathrm{x}$ & & duw & \\
\hline Veronica hederifolia & & nat & * & & $\mathrm{x}$ & & & efd & duy \\
\hline Veronica montana & & nat & * & & & & $\mathrm{x}$ & bix & \\
\hline Veronica officinalis & & nat & * & & & & & duz & \\
\hline Veronica opaca & & arc & 3 & & & & & biy & \\
\hline Veronica peregrina & & neo & * & & & & & dva & \\
\hline Veronica persica & & neo & * & & $\mathrm{x}$ & & & dvb & \\
\hline Veronica polita & & $\operatorname{arc}$ & * & & $\mathrm{x}$ & & & biz & \\
\hline Veronica praecox & & nat & 2 & $x$ & & & & bja & \\
\hline Veronica scutellata & & nat & $\mathrm{V}$ & $\hat{x}$ & & & & $\mathrm{bjb}$ & \\
\hline Veronica serpyllifolia & & nat & * & & & & & dvc & \\
\hline Veronica teucrium & & nat & $\mathrm{V}$ & $\mathrm{x}$ & & & & bjd & \\
\hline Veronica triphyllos & & arc & 3 & & & & & bje & \\
\hline Veronica verna & & nat & 2 & $\mathrm{x}$ & & & & bjf & \\
\hline Viburnum opulus & & nat & * & & & & & dve & \\
\hline Vicia angustifolia ssp. angustifolia & & arc & * & & & & & $\mathrm{dvl}$ & \\
\hline Vicia angustifolia ssp. segetalis & & neo & * & & & & & 256 & \\
\hline Vicia cassubica & & nat & 3 & $x$ & & & $x$ & bjg & \\
\hline
\end{tabular}


Table A.2 - Continued from previous page

\begin{tabular}{|c|c|c|c|c|c|c|c|c|c|}
\hline 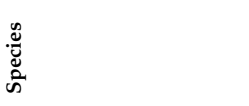 & 总 & 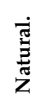 & $\begin{array}{l}\tilde{Z} \\
\vec{z}\end{array}$ & $\begin{array}{l}\stackrel{?}{\mathbf{Z}} \\
\underset{\mathbf{z}}{\mathbf{Z}}\end{array}$ & 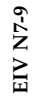 & 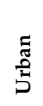 & 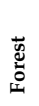 & نँ & $\begin{array}{l}\dot{\Xi} \\
\Xi\end{array}$ \\
\hline Vicia cracca & & nat & * & & & & & dvf & \\
\hline Vicia dumetorum & & nat & 3 & & & & $\mathrm{x}$ & bjh & \\
\hline Vicia hirsuta & & nat & * & & & & & dvh & \\
\hline Vicia lathyroides & & nat & 3 & $\mathrm{x}$ & & & & bji & \\
\hline Vicia pisiformis & & nat & 2 & $\mathrm{x}$ & & & $\mathrm{x}$ & bjj & \\
\hline Vicia sativa agg. & & arc & $*$ & & & & & efe & \\
\hline Vicia sepium & & nat & * & & & & & dvn & \\
\hline Vicia sylvatica & & nat & $\mathrm{V}$ & & & & $\mathrm{x}$ & dvo & \\
\hline Vicia tenuifolia & & nat & 3 & $\mathrm{x}$ & & & & bjk & \\
\hline Vicia tetrasperma & & nat & * & & & & & dvp & \\
\hline Vinca minor & & neo & $*$ & & & & $\mathrm{x}$ & dvs & \\
\hline Vincetoxicum hirundinaria & & nat & * & $\mathrm{x}$ & & & & bjl & \\
\hline Viola arvensis & & $\operatorname{arc}$ & * & & & & & dvt & \\
\hline Viola canina & & nat & $\mathrm{V}$ & $\mathrm{x}$ & & & & dvu & \\
\hline Viola hirta & & nat & * & $\mathrm{x}$ & & & & dvv & \\
\hline Viola mirabilis & & nat & 3 & & & & $\mathrm{x}$ & bjn & \\
\hline Viola odorata & & neo & * & & $\mathrm{x}$ & & $\mathrm{x}$ & dvw & \\
\hline Viola palustris & & nat & $\mathrm{V}$ & $\mathrm{x}$ & & & & $d v x$ & \\
\hline Viola persicifolia & & nat & 2 & $\mathrm{x}$ & & & & bjo & \\
\hline Viola reichenbachiana & & nat & * & & & & $\mathrm{x}$ & dvy & \\
\hline Viola riviniana & & nat & * & & & & $\mathrm{x}$ & dvz & \\
\hline Viola tricolor & & nat & * & $\mathrm{x}$ & & & & dwa & bjp \\
\hline Viola $\times$ bavarica & & nat & * & & & & $\mathrm{x}$ & dwe & \\
\hline Viscum album & & nat & * & & & & & $d w b$ & \\
\hline Vulpia bromoides & & nat & 2 & $\mathrm{x}$ & & & & bjq & \\
\hline Vulpia myuros & & nat & * & $\mathrm{x}$ & & & & dwc & \\
\hline Wolffia arrhiza & & nat & 3 & & & & & bjs & \\
\hline Woodsia ilvensis & & nat & 1 & $\mathrm{x}$ & & & & bjt & \\
\hline x Calammophila baltica & $\mathrm{x}$ & nat & * & $\mathrm{x}$ & & & & dwk & \\
\hline x Festulolium loliaceum & & nat & * & & & & & faw & \\
\hline Xanthium albinum & & neo & * & & $\mathrm{x}$ & & & $\mathrm{dwd}$ & \\
\hline Zannichellia palustris & & nat & V & & $\mathrm{x}$ & & & bju & dwg, dwh \\
\hline Zostera marina & $\mathrm{x}$ & nat & 3 & & & & & dwi & \\
\hline Zostera noltii & & nat & 3 & & & & & dwj & \\
\hline
\end{tabular}

Table A.3: Codes and full names of (sub-)species assigned to the species and/or aggregate of higher taxonomical level. Added to $=$ Code of species to which the particular (sub)species was assigned to (see Table A.2 for full names of species).

\begin{tabular}{|c|c|c|}
\hline Code & Species & Added to \\
\hline 116 & Taraxacum commixtum & bgi \\
\hline 122 & Taraxacum discretum & bgi \\
\hline 126 & Taraxacum franconicum & bgi \\
\hline 14 & Sambucus nigra $f$. laciniata & dlm \\
\hline 144 & Polygonum arenastrum & dag \\
\hline 181 & Taraxacum prionum & eex \\
\hline 182 & Taraxacum rubrisquameum & eex \\
\hline 191 & Nasturtium $\mathrm{x}$ sterile & edb \\
\hline 206 & Asplenium trichomanes ssp. pachyrachis & acw \\
\hline 224 & Asplenium trichomanes ssp. hastatum & acw \\
\hline 225 & Festuca rubra ssp. juncea & 397 \\
\hline 232 & Aster $\mathrm{x}$ salignus & ear \\
\hline 238 & Luzula luzuloides ssp. rubella & aub \\
\hline 240 & Festuca valesiaca ssp. parviflora & ecc \\
\hline 243 & Epipactis helleborine ssp. neerlandica & 370 \\
\hline 26 & Taraxacum gelertii & eex \\
\hline 260 & Trichophorum cespitosum nothossp. foersteri & bhg \\
\hline 27 & Taraxacum subalpinum & bgj \\
\hline 276 & Polypodium $\mathrm{x}$ mantoniae & edm \\
\hline 283 & Taraxacum leptoglotte & eex \\
\hline 300 & Barbarea vulgaris ssp. arcuata & ces \\
\hline 301 & Barbarea vulgaris ssp. rivularis & ces \\
\hline 34 & Taraxacum rubicundum & bgi \\
\hline 40 & Taraxacum paucilobum & bgj \\
\hline 401 & Erigeron annuus ssp. strigosus & $\operatorname{cm} x$ \\
\hline 413 & Festuca heteromalla & 397 \\
\hline
\end{tabular}

Continued on next page 
Table A.3 - Continued from previous page

\begin{tabular}{|c|c|c|}
\hline Code & Species & Added to \\
\hline 50 & Aster novae-angliae & ear \\
\hline 54 & Festuca nigrescens & 397 \\
\hline 56 & Taraxacum disseminatum & bgi \\
\hline 59 & Taraxacum bavaricum & bgj \\
\hline ace & Armeria maritima ssp. elongata & 393 \\
\hline acf & Armeria maritima ssp. halleri & 393 \\
\hline adt & Bolboschoenus maritimus & 398 \\
\hline aes & Callitriche cophocarpa & eay \\
\hline aeu & Callitriche palustris + & eay \\
\hline agy & Carex viridula var. pulchella & agm \\
\hline ahx & Cerastium pumilum & ebh \\
\hline anj & Festuca pallens & ecc \\
\hline ank & Festuca valesiaca ssp. valesiaca & ecc \\
\hline aoj & Galium verum s.l. & $\mathrm{cph}$ \\
\hline apr & Helianthemum nummularium ssp. nummularium & apq \\
\hline aps & Helianthemum nuтmularium ssp. obscurum & apq \\
\hline asf & Lamium purpureum var. incisum & ect \\
\hline ate & Lilium bulbiferum ssp. bulbiferum & atd \\
\hline atf & Lilium bulbiferum ssp. croceum & atd \\
\hline avi & Montia fontana ssp. chondrosperma & avh \\
\hline awm & Ononis repens ssp. procurrens & awn \\
\hline awo & Ononis spinosa & awn \\
\hline ayt & Plantago major ssp. winteri & edi \\
\hline bbe & Ranunculus aquatilis + & bbd \\
\hline bbg & Ranunculus peltatus ssp. baudotii & bbd \\
\hline bbi & Ranunculus circinatus & bbd \\
\hline bbj & Ranunculus fluitans & bbd \\
\hline bbk & Ranunculus hederaceus & bbd \\
\hline $\mathrm{bbm}$ & Ranunculus nemorosus & bbs \\
\hline bbn & Ranunculus ololeucos & bbd \\
\hline bbo & Ranunculus peltatus ssp. peltatus & bbd \\
\hline $\mathrm{bbq}$ & Ranunculus polyanthemoides & bbs \\
\hline bbr & Ranunculus polyanthemophyllus & bbs \\
\hline bbw & Ranunculus serpens & bbs \\
\hline bbx & Ranunculus trichophyllus & bbd \\
\hline bcq & Rosa vosagiaca agg. & dds \\
\hline bda & Salicornia europaea ssp. brachystachya & eee \\
\hline bdd & Salix repens ssp. repens & eej \\
\hline bdj & Sanguisorba minor ssp. minor & eel \\
\hline bfk & Sonchus arvensis ssp. uliginosus & eer \\
\hline bhh & Trichophorum cespitosum ssp. cespitosum & bhg \\
\hline bhi & Trichophorum cespitosum ssp. germanicum & bhg \\
\hline bif & Utricularia vulgaris + & bie \\
\hline bim & Valeriana sambucifolia & efc \\
\hline bin & Valeriana wallrothii & efc \\
\hline bjp & Viola tricolor ssp. curtisii & dwa \\
\hline cag & Achillea millefolium & eab \\
\hline can & Aethusa cynapium ssp. elata & aec \\
\hline cas & Agrostis gigantea & ead \\
\hline cay & Alchemilla filicaulis & aaq \\
\hline caz & Alchemilla glabra & aaq \\
\hline cba & Alchemilla micans & aaq \\
\hline $\mathrm{cbb}$ & Alchemilla monticola & aaq \\
\hline $\mathrm{cbc}$ & Alchemilla propinqua & aaq \\
\hline $\mathrm{cbd}$ & Alchemilla subcrenata & aaq \\
\hline cbe & Alchemilla subglobosa & aaq \\
\hline $\mathrm{cbf}$ & Alchemilla vulgaris + & aaq \\
\hline $\operatorname{cbg}$ & Alchemilla xanthochlora & aaq \\
\hline $\mathrm{cbh}$ & Alisma plantago-aquatica & eae \\
\hline $\mathrm{cbv}$ & Amaranthus blitum ssp. blitum & 363 \\
\hline cby & Amaranthus blitum ssp. emarginatus & 363 \\
\hline $\mathrm{cbz}$ & Amaranthus powellii & eag \\
\hline $\operatorname{ccx}$ & Anthyllis vulneraria ssp. maritima & 391 \\
\hline cсу & Anthyllis vulneraria ssp. pseudovulneraria & 391 \\
\hline cdl & Arenaria serpyllifolia ssp. serpyllifolia & ean \\
\hline $\mathrm{cdm}$ & Armeria maritima ssp. maritima & 393 \\
\hline $\mathrm{cdw}$ & Aster lanceolatus & ear \\
\hline $\mathrm{cdx}$ & Aster novi-belgii + & ear \\
\hline cdy & Aster parviflorus & ear \\
\hline cei & Atriplex prostrata & eas \\
\hline сер & Ballota nigra ssp. meridionalis & adj \\
\hline ceq & Ballota nigra ssp. nigra & adj \\
\hline cey & Betula pubescens ssp. carpatica & eat \\
\hline cez & Betula pubescens ssp. pubescens & eat \\
\hline $\mathrm{cfm}$ & Bromus hordeaceus & eau \\
\hline $\mathrm{cfz}$ & Callitriche hamulata & eay \\
\hline
\end{tabular}


Table A.3 - Continued from previous page

\begin{tabular}{|c|c|c|}
\hline Code & Species & Added to \\
\hline cga & Callitriche obtusangula & eay \\
\hline $\mathrm{cgb}$ & Callitriche platycarpa & eay \\
\hline $\mathrm{cgc}$ & Callitriche stagnalis & eay \\
\hline $\operatorname{cgq}$ & Cardamine pratensis & eaz \\
\hline cgy & Carex arenaria & $\mathrm{ebc}$ \\
\hline $\mathrm{chf}$ & Carex guestphalica & ebe \\
\hline chk & Carex pairae & ebe \\
\hline chs & Carex spicata & ebe \\
\hline chw & Carex vulpina & ebg \\
\hline cih & Centaurium littorale ssp. littorale & ahn \\
\hline $\mathrm{cii}$ & Centaurium littorale ssp. uliginosum & $\mathrm{ahn}$ \\
\hline cjc & Chenopodium rubrum & ebj \\
\hline cjd & Chenopodium strictum & ebi \\
\hline cje & Chenopodium suecicum & ebi \\
\hline cka & Crataegus laevigata ssp. laevigata & ebl \\
\hline $\mathrm{ckb}$ & Crataegus laevigata ssp. palmstruchii & ebl \\
\hline cke & Crataegus monogyna ssp. monogyna & ebo \\
\hline $\mathrm{ckf}$ & Crataegus monogyna ssp. nordica & ebo \\
\hline $\mathrm{cmh}$ & Epilobium tetragonum ssp. lamyi & ebw \\
\hline $\mathrm{cmj}$ & Epipactis helleborine ssp. helleborine & 370 \\
\hline $\mathrm{cmk}$ & Epipactis leptochila ssp. leptochila & amj \\
\hline $\mathrm{cml}$ & Epipactis leptochila ssp. neglecta & amj \\
\hline $\mathrm{cng}$ & Euphrasia micrantha & anf \\
\hline $\mathrm{cnr}$ & Euphrasia nemorosa + & anf \\
\hline $\operatorname{cnx}$ & Festuca filiformis & ecc \\
\hline $\mathrm{cnz}$ & Festuca guestfalica & ecc \\
\hline coa & Festuca ovina + & ecc \\
\hline $\operatorname{cod}$ & Festuca rubra ssp. arenaria & 397 \\
\hline coe & Festuca rubra ssp. litoralis & 397 \\
\hline $\operatorname{cog}$ & Festuca rupicola & ecc \\
\hline $\mathrm{coh}$ & Festuca brevipila & ecc \\
\hline coi & Filipendula ulmaria var. denudata & ece \\
\hline coj & Filipendula ulmaria var. ulmaria & ece \\
\hline $\operatorname{coq}$ & Fumaria officinalis ssp. wirtgenii & ecg \\
\hline $\operatorname{csz}$ & Lamium galeobdolon & ecq \\
\hline cta & Lamium montanum & ecq \\
\hline $\operatorname{ctr}$ & Leonurus cardiaca ssp. cardiaca & asy \\
\hline cts & Leonurus cardiaca ssp. villosus & asy \\
\hline ctx & Galium palustre ssp. elongatum & $\mathrm{cpc}$ \\
\hline cub & Leucanthemum ircutianum & ecu \\
\hline cwp & Monotropa hypophegea & avg \\
\hline cwq & Monotropa hypopitys + & avg \\
\hline cwr & Montia fontana ssp. amporitana & avh \\
\hline cws & Montia fontana ssp. fontana & avh \\
\hline cwt & Montia fontana ssp. variabilis & avh \\
\hline $\operatorname{cxg}$ & Nasturtium microphyllum & edb \\
\hline cyi & Papaver dubium ssp. dubium & ede \\
\hline суј & Papaver dubium ssp. lecoqii & ede \\
\hline cyk & Polygala vulgaris ssp. collina & aze \\
\hline cyg & Polygala vulgaris ssp. oxyptera & aze \\
\hline cza & Phyteuma spicatum ssp. coeruleum & ayo \\
\hline $\mathrm{czb}$ & Phyteuma spicatum ssp. spicatum & ayo \\
\hline czk & Plantago major ssp. intermedia & edi \\
\hline $\mathrm{czl}$ & Plantago major ssp. major & edi \\
\hline daa & Polygala amara ssp. brachyptera & $\mathrm{azc}$ \\
\hline dab & Polygala amarella & azc \\
\hline dal & Persicaria lapathifolia ssp. brittingeri & edl \\
\hline dam & Persicaria lapathifolia ssp. pallida & edl \\
\hline dar & Polypodium interjectum & edm \\
\hline daw & Potamogeton berchtoldii & edo \\
\hline dco & Ranunculus penicillatus & bbd \\
\hline ddf & Rosa caesia & $\mathrm{bcl}$ \\
\hline ddm & Rosa pseudoscabriuscula & bcp \\
\hline ddn & Rosa sherardii & bcp \\
\hline $\mathrm{ddq}$ & Rosa tomentosa + & bcp \\
\hline ddr & Rosa villosa & bcp \\
\hline $\mathrm{dkh}$ & Sagina micropetala & eed \\
\hline $\mathrm{dkn}$ & Salicornia europaea ssp. europaea & eee \\
\hline $\mathrm{dkq}$ & Salix alba ssp. vitellina & eeg \\
\hline $\mathrm{dkv}$ & Salix fragilis + & eeh \\
\hline $\mathrm{dky}$ & Salix purpurea ssp. lambertiana & eei \\
\hline dla & Salix repens ssp. dunensis & eej \\
\hline dlb & Salix triandra ssp. amygdalina & eek \\
\hline dle & Salix $\mathrm{x}$ rubens & eeh \\
\hline dlg & Salsola kali ssp. kali & bdf \\
\hline dlh & Salsola kali ssp. tragus & bdf \\
\hline
\end{tabular}


Table A.3 - Continued from previous page

\begin{tabular}{lll}
\hline Code & Species & Added to \\
\hline $\mathrm{dlw}$ & Scleranthus polycarpos & een \\
$\mathrm{dmd}$ & Sedum maximum & eeo \\
$\mathrm{dme}$ & Sedum telephium + & eeo \\
$\mathrm{dnm}$ & Solanum nigrum ssp. schultesii & eeq \\
$\mathrm{dnw}$ & Sonchus arvensis ssp. arvensis & eer \\
$\mathrm{doc}$ & Sparganium erectum ssp. neglectum & $\mathrm{ees}$ \\
$\mathrm{dpo}$ & Taraxacum balticum & $\mathrm{bgj}$ \\
$\mathrm{dpq}$ & Taraxacum brachyglossum & $\mathrm{bgi}$ \\
$\mathrm{dpr}$ & Taraxacum bracteatum & $\mathrm{eex}$ \\
$\mathrm{dpx}$ & Bolboschoenus maritimus x yagara & 398 \\
$\mathrm{dqj}$ & Taraxacum haematicum & $\mathrm{eex}$ \\
$\mathrm{dqv}$ & Taraxacum lacistophyllum & $\mathrm{bgi}$ \\
$\mathrm{drg}$ & Taraxacum nordstedtii & $\mathrm{eex}$ \\
$\mathrm{drn}$ & Taraxacum palustre & $\mathrm{bgj}$ \\
$\mathrm{drw}$ & Taraxacum proximum & $\mathrm{bgi}$ \\
$\mathrm{dsa}$ & Taraxacum duplidentifrons & $\mathrm{eex}$ \\
$\mathrm{dsd}$ & Taraxacum scanicum & $\mathrm{bgi}$ \\
$\mathrm{dsg}$ & Taraxacum parnassicum & $\mathrm{bgi}$ \\
$\mathrm{dsr}$ & Taraxacum tenuilobum & $\mathrm{bgi}$ \\
$\mathrm{dss}$ & Taraxacum tortilobum & $\mathrm{bgi}$ \\
$\mathrm{dsx}$ & Festuca polesica & $\mathrm{ecc}$ \\
$\mathrm{duu}$ & Veronica catenata & $\mathrm{biv}$ \\
$\mathrm{duy}$ & Veronica hederifolia ssp. lucorum & $\mathrm{efd}$ \\
$\mathrm{dwg}$ & Zannichellia palustris ssp. palustris & $\mathrm{bju}$ \\
$\mathrm{dwh}$ & Zannichellia palustris ssp. pedicellata & $\mathrm{bju}$ \\
$\mathrm{eah}$ & Anagallis arvensis var. caerulea & $\mathrm{cck}$ \\
$\mathrm{eap}$ & Asplenium trichomanes ssp. trichomanes & $\mathrm{acw}$ \\
$\mathrm{ebk}$ & Crataegus curvisepala agg. & $\mathrm{cjz}$ \\
$\mathrm{ebm}$ & Crataegus laevigata & $\mathrm{ebl}$ \\
$\mathrm{ebq}$ & Dactylorhiza fuchsii & $\mathrm{akr}$ \\
$\mathrm{ebu}$ & Eleocharis palustris ssp. palustris & $\mathrm{clr}$ \\
$\mathrm{ebv}$ & Eleocharis palustris ssp. vulgaris & $\mathrm{clr}$ \\
$\mathrm{ecd}$ & Festuca rubra & 397 \\
$\mathrm{ecm}$ & Hypericum maculatum ssp. obtusiusculum & $\mathrm{crp}$ \\
\hline & & \\
& & \\
\hline
\end{tabular}

Table A.4: Soil types listed in the soil map of Lower Saxony (BÜK 50) and the assignment to 27 soil type categories. Abbr. = Abbreviation of soil type.

\begin{tabular}{llr}
\hline Abbr. & Soil type & Category \\
\hline AB & Vega & 1 \\
AB-S & Vega-Pseudogley & 1 \\
B-P & Braunerde-Podsol & 2 \\
B & Braunerde & 2 \\
B-Q & Braunerde-Regosol & 2 \\
B-D & Braunerde-Pelosol & 2 \\
B-S & Braunerde-Pseudogley & 2 \\
B-R & Braunerde-Rendzina & 2 \\
B-pL & Braunerde-Parabraunerde; podsoliert & 2 \\
B-N & Braunerde-Ranker & 2 \\
B-Z & Braunerde-Pararendzina & 2 \\
B-pQ & Braunerde-Regosol; podsoliert & 2 \\
B-L & Braunerde-Parabraunerde & 2 \\
D & Pelosol & 3 \\
D-S & Pelosol-Pseudogley & 3 \\
D-B & Pelosol-Braunerde & 3 \\
E//P-G & Plaggenesch unterlagert von Podsol-Gley & 4 \\
E//P & Plaggenesch unterlagert von Podsol & 4 \\
E/G-P & Gley-Podsol mit Plaggenauflage & 4 \\
\hline
\end{tabular}


Table A.4 - Continued from previous page

\begin{tabular}{|c|c|c|}
\hline Abbr. & Soil type & Category \\
\hline $\mathrm{E} / / \mathrm{G}$ & Plaggenesch unterlagert von Gley & 4 \\
\hline E/S-P & Pseudogley-Podsol mit Plaggenauflage & 4 \\
\hline $\mathrm{E} / / \mathrm{S}$ & Plaggenesch unterlagert von Pseudogley & 4 \\
\hline $\mathrm{E} / / \mathrm{S}-\mathrm{B}$ & Plaggenesch unterlagert von Pseudogley-Braunerde & 4 \\
\hline $\mathrm{E} / / \mathrm{B}$ & Plaggenesch unterlagert von Braunerde & 4 \\
\hline $\mathrm{E} / \mathrm{P}-\mathrm{B}$ & Podsol-Braunerde mit Plaggenauflage & 4 \\
\hline $\mathrm{E}$ & Plaggenesch & 4 \\
\hline $\mathrm{E} / \mathrm{S}-\mathrm{B}$ & Pseudogley-Braunerde mit Plaggenauflage & 4 \\
\hline $\mathrm{E} / \mathrm{B}$ & Braunerde mit Plaggenauflage & 4 \\
\hline $\mathrm{E} / / \mathrm{G}-\mathrm{B}$ & Plaggenesch unterlagert von Gley-Braunerde & 4 \\
\hline $\mathrm{E} / \mathrm{G}-\mathrm{B}$ & Gley-Braunerde mit Plaggenauflage & 4 \\
\hline $\mathrm{E} / / \mathrm{B}-\mathrm{G}$ & Plaggenesch unterlagert von Braunerde-Gley & 4 \\
\hline $\mathrm{E} / / \mathrm{S}-\mathrm{L}$ & Plaggenesch unterlagert von Pseudogley-Parabraunerde & 4 \\
\hline $\mathrm{E} / \mathrm{L}$ & Parabraunerde mit Plaggenauflage & 4 \\
\hline $\mathrm{E} / / \mathrm{L}$ & Plaggenesch unterlagert von Parabraunerde & 4 \\
\hline $\mathrm{E} / \mathrm{S}$ & Pseudogley mit Plaggenauflage & 4 \\
\hline $\mathrm{E} / \mathrm{P}$ & Podsol mit Plaggenauflage & 4 \\
\hline $\mathrm{E} / / \mathrm{P}-\mathrm{B}$ & Plaggenesch unterlagert von Podsol-Braunerde & 4 \\
\hline $\mathrm{E} / / \mathrm{S}-\mathrm{P}$ & Plaggenesch unterlagert von Pseudogley-Podsol & 4 \\
\hline G & Gley & 5 \\
\hline G-P & Gley-Podsol & 5 \\
\hline G-S & Gley-Pseudogley & 5 \\
\hline G-Q & Gley-Regosol & 5 \\
\hline $\mathrm{G}-\mathrm{AB}$ & Gley-Vega & 5 \\
\hline G-B & Gley-Braunerde & 5 \\
\hline G-T & Gley-Schwarzerde & 5 \\
\hline G-OL & Gley-Lockersyrosem & 5 \\
\hline G-P-S & Gley-Podsol-Pseudogley & 5 \\
\hline G-L & Gley-Parabraunerde & 5 \\
\hline G-Z & Gley-Pararendzina & 5 \\
\hline $\mathrm{HHv}$ & Erd-Hochmoor & 6 \\
\hline $\mathrm{HNv} / \mathrm{G}$ & Gley mit Erd-Niedermoorauflage & 6 \\
\hline $\mathrm{HNv}$ & Erd-Niedermoor & 6 \\
\hline $\mathrm{HHv} / \mathrm{G}-\mathrm{P}$ & Gley-Podsol mit Erd-Hochmoorauflage & 6 \\
\hline $\mathrm{HH}$ & Hochmoor & 6 \\
\hline $\mathrm{HHv} / / \mathrm{S}-\mathrm{G}$ & Erd-Hochmoor unterlagert von Pseudogley-Gley & 6 \\
\hline $\mathrm{HHv} / \mathrm{G}$ & Gley mit Erd-Hochmoorauflage & 6 \\
\hline $\mathrm{K} / / \mathrm{G}$ & Kolluvisol unterlagert von Gley & 7 \\
\hline $\mathrm{K} / / \mathrm{L}$ & Kolluvisol unterlagert von Parabraunerde & 7 \\
\hline $\mathrm{K} / / \mathrm{S}-\mathrm{L}$ & Kolluvisol unterlagert von Pseudogley-Parabraunerde & 7 \\
\hline $\mathrm{K} / / \mathrm{B}$ & Kolluvisol unterlagert von Braunerde & 7 \\
\hline $\mathrm{K}$ & Kolluvisol & 7 \\
\hline $\mathrm{L}$ & Parabraunerde & 8 \\
\hline L-S & Parabraunerde-Pseudogley & 8 \\
\hline L-SH & Parabraunerde-Haftäõssepseudogley & 8 \\
\hline $\mathrm{pL}$ & Parabraunerde; podsoliert & 8 \\
\hline $\mathrm{MC}$ & Kalkmarsch & 9 \\
\hline MD & Dwogmarsch & 10 \\
\hline
\end{tabular}


Table A.4 - Continued from previous page

\begin{tabular}{|c|c|c|}
\hline Abbr. & Soil type & Category \\
\hline $\mathrm{MK} / / \mathrm{HN}$ & Knickmarsch unterlagert von Niedermoor & 11 \\
\hline MK & Knickmarsch & 11 \\
\hline $\mathrm{MK} / \mathrm{G}$ & Gley mit Knickauflage & 11 \\
\hline $\mathrm{MK} / / \mathrm{HH}$ & Knickmarsch unterlagert von Hochmoor & 11 \\
\hline $\mathrm{MN}$ & Kleimarsch & 12 \\
\hline $\mathrm{MN} / / \mathrm{HN}$ & Kleimarsch unterlagert von Niedermoor & 12 \\
\hline $\mathrm{MN} / \mathrm{HN}$ & Niedermoor mit Knickmarschauflage & 12 \\
\hline $\mathrm{MN} / / \mathrm{HH}$ & Kleimarsch unterlagert von Hochmoor & 12 \\
\hline $\mathrm{MN} / \mathrm{HH}$ & Hochmoor mit Knickmarschauflage & 12 \\
\hline $\mathrm{MO}$ & Organomarsch & 13 \\
\hline $\mathrm{MO} / / \mathrm{HN}$ & Organomarsch unterlagert von Niedermoor & 13 \\
\hline MR & Rohmarsch & 14 \\
\hline $\mathrm{MR} / \mathrm{HN}$ & Niedermoor mit Organomarschauflage & 14 \\
\hline $\mathrm{MR} / \mathrm{HH}$ & Hochmoor mit Rohmarschauflage & 14 \\
\hline $\mathrm{N}$ & Ranker & 15 \\
\hline $\mathrm{O}$ & Syrosem & 16 \\
\hline OL & Lockersyrosem & 16 \\
\hline P-G & Podsol-Gley & 17 \\
\hline $\mathrm{P}$ & Podsol & 17 \\
\hline P-B & Podsol-Braunerde & 17 \\
\hline P-S & Podsol-Pseudogley & 17 \\
\hline $\mathrm{P}-\mathrm{Q}$ & Podsol-Regosol & 17 \\
\hline$Q^{2}$ & Regosol & 18 \\
\hline $\mathrm{pQ}$ & podsoliger Regosol & 18 \\
\hline S-P & Pseudogley-Podsol & 19 \\
\hline S-B & Pseudogley-Braunerde & 19 \\
\hline S & Pseudogley & 19 \\
\hline S-G & Pseudogley-Gley & 19 \\
\hline S-L & Pseudogley-Parabraunerde & 19 \\
\hline S-T & Pseudogley-Schwarzerde & 19 \\
\hline $\mathrm{S} / / \mathrm{MN}$ & Pseudogley unterlagert von Kleimarsch & 19 \\
\hline $\mathrm{S}-\mathrm{AB}$ & Pseudogley-Vega & 19 \\
\hline S-D & Pseudogley-Pelosol & 19 \\
\hline S-K & Pseudogley-Kolluvisol & 19 \\
\hline pS & Pseudogley; podsoliert & 19 \\
\hline $\mathrm{SH}$ & Haftnõssepseudogley & 19 \\
\hline S-Q & Pseudogley-Regosol & 19 \\
\hline $\mathrm{R}$ & Rendzina & 20 \\
\hline $\mathrm{F}$ & Felshumusboden & 21 \\
\hline $\mathrm{T}-\mathrm{L}$ & Schwarzerde-Parabraunerde & 22 \\
\hline $\mathrm{T}$ & Schwarzerde & 22 \\
\hline $\mathrm{YD} \backslash \mathrm{HH}$ & Hochmoor mit Deckkulturbodenauflage & 23 \\
\hline $\mathrm{YD} / \mathrm{HH}$ & Hochmoor mit Deckkulturbodenauflage & 23 \\
\hline YT & Spittmarsch & 24 \\
\hline YUhn & Tiefumbruchboden & 25 \\
\hline YUp & Tiefumbruchboden & 25 \\
\hline YUg & Tiefumbruchboden & 25 \\
\hline YUhh & Tiefumbruchboden & 25 \\
\hline
\end{tabular}


Table A.4 - Continued from previous page

\begin{tabular}{llr}
\hline Abbr. & Soil type & Category \\
\hline YUg-p & Tiefumbruchboden & 25 \\
YUh & Tiefumbruchboden & 25 \\
YUs-p & Tiefumbruchboden & 25 \\
YUmn & Tiefumbruchboden & 25 \\
YUab & Tiefumbruchboden & 25 \\
YUs-ab & Tiefumbruchboden & 25 \\
YUg-ab & Tiefumbruchboden & 25 \\
YUs & Tiefumbruchboden & 25 \\
YUs-g & Tiefumbruchboden & 25 \\
Z & Pararendzina & 26 \\
ZS & Strand & 27 \\
\hline
\end{tabular}

Table A.5: Roads that are represented as polylines in the ATKIS-DLM were buffered by using the buffer distance specified below. Standard values for the width of roads was taken from FGSV (1996). $\mathrm{n} / \mathrm{a}=$ not available.

\begin{tabular}{|c|c|c|}
\hline Road category & FGSV (1996) & Buffer \\
\hline $\begin{array}{l}\text { Motorways } \\
\text { (Autobahnen) }\end{array}$ & $\begin{array}{l}\text { Minimum: } 20 \mathrm{~m} \\
\text { Maximum: } 29 \mathrm{~m} \\
\text { Standard: } 23 \mathrm{~m}\end{array}$ & $\begin{array}{l}11.5 \mathrm{~m} \\
\rightarrow 2 \times 11.5 \mathrm{~m}=23 \mathrm{~m} \text { width }\end{array}$ \\
\hline $\begin{array}{l}\text { Federal highways } \\
\text { (Bundesstraßen) }\end{array}$ & $\begin{array}{l}\text { Minimum: } 7.5 \mathrm{~m} \\
\text { Maximum: } 11.5 \mathrm{~m}, 15 \mathrm{~m} \text { for } \\
\text { twin-track roads } \\
\text { Standard: } \mathrm{n} / \mathrm{a} .\end{array}$ & $\begin{array}{l}5.625 \mathrm{~m} \\
\rightarrow 2 \times 5.625 \mathrm{~m}=11.25 \mathrm{~m} \text { width } \\
\text { (average of } 7.5 \mathrm{~m} \text { and } 15 \mathrm{~m} \text { ) }\end{array}$ \\
\hline $\begin{array}{l}\text { State roads } \\
\text { (Landesstraßen) }\end{array}$ & Standard: $6.5 \mathrm{~m}$ & $\begin{array}{l}3.25 \mathrm{~m} \\
\rightarrow 2 \times 3.25 \mathrm{~m}=6.5 \mathrm{~m} \text { width }\end{array}$ \\
\hline $\begin{array}{l}\text { County roads } \\
\text { (Kreisstraßen) }\end{array}$ & $\begin{array}{l}\mathrm{n} / \mathrm{a} \text {; standard of } 6.5 \mathrm{~m} \text { as- } \\
\text { sumed }\end{array}$ & $\begin{array}{l}3.25 \mathrm{~m} \\
\rightarrow 2 \times 3.25 \mathrm{~m}=6.5 \mathrm{~m} \text { width }\end{array}$ \\
\hline $\begin{array}{l}\text { Municipal roads } \\
\text { (Gemeindestraßen) }\end{array}$ & $\begin{array}{l}\mathrm{n} / \mathrm{a} \text {; standard of } 5.5 \mathrm{~m} \text { as- } \\
\text { sumed }\end{array}$ & $\begin{array}{l}2.75 \mathrm{~m} \\
\rightarrow \rightarrow 2 \times 2.75 \mathrm{~m}=5.5 \mathrm{~m} \text { width }\end{array}$ \\
\hline
\end{tabular}


Table A.6: Land cover types (LCT) used for the calculation of landscape metrics, their description as well as the ATKIS-DLM objects included in the particular LCT.

\begin{tabular}{|c|c|c|}
\hline LCT & Description & Objects ATKIS-DLM \\
\hline $\begin{array}{l}\text { Industrial ar- } \\
\text { eas }\end{array}$ & $\begin{array}{l}\text { Areas used for (non } \\
\text { agricultural) production } \\
\text { (e.g. supply and dis- } \\
\text { posal facilities, exhibi- } \\
\text { tion centers, mining ar- } \\
\text { eas etc. }\end{array}$ & $\begin{array}{l}\text { Abfallbeseitigungsanlage, Abfalldeponie, Abset- } \\
\text { zbecken/ Rieselfeld/ Schlammteich, Ausstellungs- } \\
\text { gelände/ Messegelände, Bergbaubetrieb, Fläche beson- } \\
\text { derer funktionaler Prägung, Förderanlage, Freileitung, } \\
\text { Gärtnerei, Heizwerk, Industrie- und Gewerbefläche, } \\
\text { Kläranlage/Klärwerk, Kraftwerk, Raffinerie, Tagebau/ } \\
\text { Grube/ Steinbruch, Umspannwerk, Wasserwerk, Werft, } \\
\text { Dock, Hafenbecken }\end{array}$ \\
\hline Urban areas & $\begin{array}{l}\text { Areas used for hous- } \\
\text { ing and recreation (e.g. } \\
\text { residential areas, parks, } \\
\text { campgrounds etc.) }\end{array}$ & $\begin{array}{l}\text { Campingplatz, Denkmal/ Denkstein/ Standbild, } \\
\text { Fläche gemischter Nutzung, Freilichtmuseum, } \\
\text { Freilichttheater, Freizeitanlage, Freizeitpark/ Sa- } \\
\text { faripark, Friedhof, Gartenland, Golfplatz, Grünan- } \\
\text { lage, Ortslage, Schiesstand, Schwimmbad/ Freibad, } \\
\text { Schwimmbecken, Spielfeld/ Spielfläche, Sportanlage, } \\
\text { Stadion, Wohnbaufläche, Zoo, Zuschauertribüne }\end{array}$ \\
\hline Traffic areas & $\begin{array}{l}\text { Areas used for transport } \\
\text { (roads, railway lines, air- } \\
\text { ports, etc.) }\end{array}$ & $\begin{array}{l}\text { Bahnhofsanlage, Bahnkörper, Bahnstrecke, Brücke/ } \\
\text { Unterführung/ Überführung, Fahrbahn, Flughafen, } \\
\text { Flughafen-Vorfeld, Flugplatz/ Landeplatz, Hafen, } \\
\text { Hafenbecken, Platz (Rastplatz/ Parkplatz), Raststätte, } \\
\text { Rollbahn, Schienenbahn, Schleuse, Schleusenkammer, } \\
\text { Straße, Straßenkörper, Tunnel, Weg }\end{array}$ \\
\hline Croplands & $\begin{array}{l}\text { Areas used for agricul- } \\
\text { tural production }\end{array}$ & Ackerland, Rieselfeld, Sonderkultur \\
\hline Forests & Forested areas & Wald/ Forst \\
\hline Grasslands & Grassland areas & Grünland \\
\hline Water bodies & $\begin{array}{l}\text { Standing and flow- } \\
\text { ing waters (e.g. lakes, } \\
\text { streams, channels, } \\
\text { springs) }\end{array}$ & $\begin{array}{l}\text { Binnensee/ Stausee/ Teich, Graben/ Kanal (Wasser- } \\
\text { wirtschaft), Kanal (Schifffahrt), Strom/ Fluss/ Bach, } \\
\text { Talsperre/ Wehr, Quelle }\end{array}$ \\
\hline Others & $\begin{array}{l}\text { Areas not belonging to } \\
\text { any of the above men- } \\
\text { tioned LCT (e.g. tree } \\
\text { rows, bogs, islands) }\end{array}$ & $\begin{array}{l}\text { Fläche z.Zt. unbestimmbar, Grenze, Halde, Aufschüt- } \\
\text { tung, Nasser Boden, Vegetationslose Fläche, Zaun, } \\
\text { Uferbefestigung, Heide, Baumgruppe/ Baumreihe, } \\
\text { Gehölz, Moor, Insel, Meer, Priel, Sandbank, Watt }\end{array}$ \\
\hline
\end{tabular}




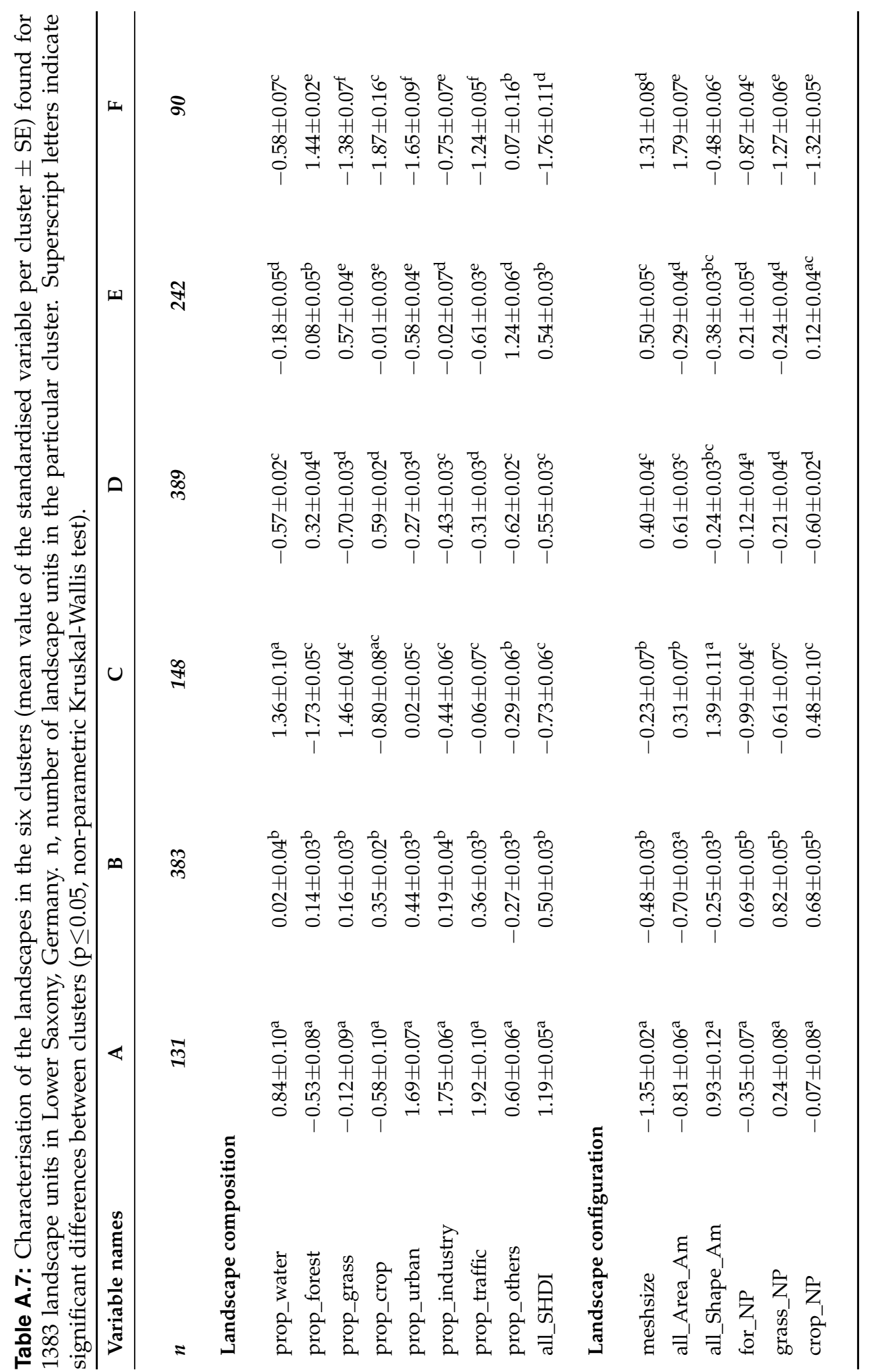



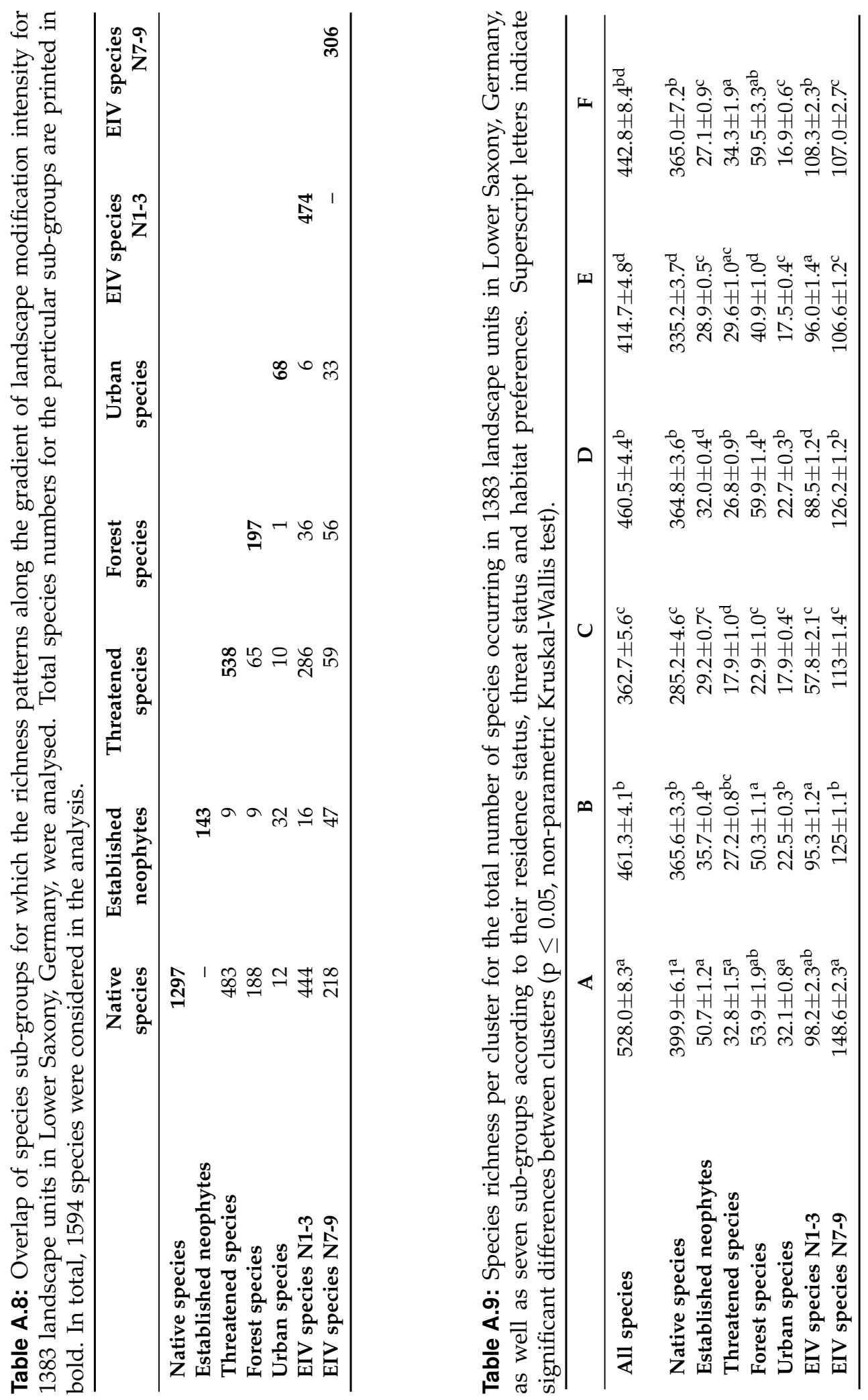


\section{References}

BfN (Bundesamt für Naturschutz) (2013): FloraWeb: Daten und Informationen zu Wildpflanzen und zur Vegetation Deutschlands. URL http:/ / www.floraweb.de. Retrieved 07/01/2013.

FGSV (Forschungsgesellschaft FÜR Strassen- UND VerKeHRSWESEN) (1996): Richtlinien für die Anlage von Straßen, Teil: Querschnitte (RAS-Q 96).

Garve, E. (2004): Rote Liste und Florenliste der Farn- und Blütenpflanzen in Niedersachsen und Bremen. Informationsdienst Naturschutz Niedersachsen 24: 1-76.

KLOTZ, S.; KüHN, I. \& DuRKA, W. (2002): BIOLFLOR - Eine Datenbank zu biologischökologischen Merkmalen der Gefäßpflanzen in Deutschland. Schriftenreihe für Vegetationskunde 38: 1-334. URL http://www.biolflor.de.

Pilgrim, B. \& Franke, R. (1993): Kartographische Arbeitsgrundlage für faunistische und floristische Erfassungen. Naturschutz und Landschaftspflege in Niedersachsen A/5. Niedersächsisches Landesamt für Ökologie.

SChmidt, M.; KRIEbitzsch, W.-U. \& EwAld, J. (2011): Waldartenlisten der Farn- und Blütenpflanzen, Moose und Flechten Deutschlands. BfN-Skripten 299: 1-111. 


\section{Appendix B}

The contents of Appendix B can be found on the DVD accompanying this thesis.

\section{PDF file of the present dissertation}

\section{Diss_Schmiedel.pdf}

Schmiedel, I. (2014): Vascular plant species richness at the landscape scale: Patterns and processes. Dissertation. Georg-August-University of Göttingen. 218 pp.

\section{PDF files of publications related to this thesis}

\section{Schmidt_et_al_2014.pdf}

Schmidt, M; Mölder, A.; Schönfelder, E.; Engel, F.; Schmiedel, I. \& Culmsee, H. (2014): Determining ancient woodland indicator plants for practical use: A new approach developed in northwest Germany. Forest Ecology and Management 330: 228-239.

\section{Culmsee_et_al_2014.pdf}

Culmsee, H.; Schmidt, M.; Schmiedel, I.; Schacherer, A.; Meyer, P. \& Leuschner, C. (2014): Predicting the distribution of forest habitat types using indicator species to facilitate systematic conservation planning. Ecological Indicators 37: 131-144.

\section{Schmiedel_et_al_2013.pdf}

Schmiedel, I.; Schmidt, M.; Schacherer, A. \& Culmsee, H. (2013): Die Effektivität von Schutzgebieten für die Erhaltung seltener und gefährdeter Gefäßpflanzenarten. Eine Untersuchung im niedersächsischen Tiefland. Naturschutz und Landschaftsplanung 45: 45-52. 


\section{Schmidt_et_al_2011.pdf}

Schmidt, M.; Culmsee, H.; Boch, S.; Heinken, T.; Müller, J. \& Schmiedel, I. (2011): Anwendungsmöglichkeiten von Waldartenlisten für Gefäßpflanzen, Moose und Flechten. BfN-Skripten 299: 25-45.

\section{Schmiedel_et_al_2011.pdf}

Schmiedel, I.; Schacherer, A.; Hauck, M.; Schmidt, M. \& Culmsee, H. (2011): Verbreitungsmuster der Farn- und Blütenpflanzen in Niedersachsen und Bremen unter Berücksichtigung ihres Einbürgerungsstatus und ihrer Gefährdungssituation. Tuexenia 31: 211-226. 